\title{
Clinically relevant and economic outcomes of maintenance pharmacotherapy in chronic obstructive pulmonary disease (COPD)
}

\author{
Anna O. D'Souza \\ West Virginia University
}

Follow this and additional works at: https://researchrepository.wvu.edu/etd

\author{
Recommended Citation \\ D'Souza, Anna O., "Clinically relevant and economic outcomes of maintenance pharmacotherapy in \\ chronic obstructive pulmonary disease (COPD)" (2006). Graduate Theses, Dissertations, and Problem \\ Reports. 2433. \\ https://researchrepository.wvu.edu/etd/2433
}

This Dissertation is protected by copyright and/or related rights. It has been brought to you by the The Research Repository @ WVU with permission from the rights-holder(s). You are free to use this Dissertation in any way that is permitted by the copyright and related rights legislation that applies to your use. For other uses you must obtain permission from the rights-holder(s) directly, unless additional rights are indicated by a Creative Commons license in the record and/ or on the work itself. This Dissertation has been accepted for inclusion in WVU Graduate Theses, Dissertations, and Problem Reports collection by an authorized administrator of The Research Repository @ WVU. For more information, please contact researchrepository@mail.wvu.edu. 
Clinically Relevant and Economic Outcomes of Maintenance Pharmacotherapy in Chronic Obstructive Pulmonary Disease (COPD)

Anna O. D’Souza

Dissertation Submitted to the

School of Pharmacy at

West Virginia University

in partial fulfillment of the requirements for the degree of

\author{
Doctor of Philosophy \\ in \\ Pharmaceutical Sciences
}

Michael J. Smith, PhD, RPh Chair

Lesley-Ann Miller, PhD

S. Suresh Madhavan, PhD, MBA

Roberto Benzo, MD, MSc

Laura A. Menditto, MPH, MBA

Department of Pharmaceutical Systems \& Policy

Morgantown, West Virginia

2006

Keywords: COPD, Medicaid, and Inhaled corticosteroids

Copyright 2006 Anna O. D’Souza 


\title{
ABSTRACT \\ Clinically Relevant and Economic Outcomes of Maintenance Pharmacotherapy in Chronic Obstructive Pulmonary Disease (COPD)
}

\begin{abstract}
Anna O. D’Souza
COPD is ranked as the fourth leading cause of death with an estimated cost of $\$ 37.2$ billion in the U.S. primarily due to exacerbations. Therefore, the Global Initiative for Obstructive Lung Disease (GOLD) committee has identified a need for assessing the effect of drug therapies on outcomes of exacerbations, costs, and mortality. This study used Medicaid data from West Virginia and Kentucky, and involved three phases. Phase I assessed the costs incurred due to COPD, and the additional cost burden imposed by a concomitant diagnosis of asthma. Phase II compared the risk and frequency of exacerbations and COPD-related costs of currently available drug therapies. Phase III assessed the effect of inhaled corticosteroids (ICS) on mortality using two different study designs, and also tested if this effect was mediated by the effect of ICS on severe exacerbations. Results of Phase I showed that recipients with COPD incurred 1.5 times significantly higher healthcare costs than recipients without COPD, primarily due to differences in hospitalization and ER costs. Asthma was found to increase the COPD-related cost of a recipient with COPD by $50 \%$. Results for Phase II showed no differences in reducing exacerbations and total COPD-related costs in a relatively less severe COPD population between recipients treated with the combination product of ipratropium/albuterol versus inhaled long-acting beta-agonists. Phase II also showed that therapy with ICS either alone or in combination with an inhaled long-acting beta-agonist did not reduce the risk of a severe exacerbation or the frequency of moderate exacerbations when compared to therapy with short-acting bronchodilators or with an inhaled long-acting beta-agonist. However, recipients initiated on ICS either alone or in combination with an inhaled long-acting beta-agonist had higher total COPD-related costs driven mainly by higher COPD-related prescription drug costs. In Phase III, recipients initiated on ICS had a $40 \%$ reduction in mortality using the case-control design. The cohort design found a relative risk reduction of $48 \%$, and an absolute risk reduction of $1.7 \%$ corresponding to a value of 59 for number needed to treat. The effect of ICS on mortality was not mediated by the effect of ICS on severe exacerbations.
\end{abstract}


For Mummy \& Daddy 


\section{ACKNOWLEDGEMENTS}

The successful completion of my doctoral degree would not have been possible without the kind support and invaluable guidance of many individuals, and I would like to take this opportunity to extend my sincere gratitude to them. Firstly, I give special thanks to the Good Lord above for always watching over me. Secondly, I would like to thank my advisor, Dr. Michael Smith (Mike) for being very ${ }^{\mathrm{n}}$ patient during the course of my study. I can truly say that I would not be the independent and strong researcher I am today without his guidance. My sincere thanks to my other committee members for their support at various stages in my graduate career: Dr Lesley-Ann Miller for her constant encouragement that never let me give up; Dr. Suresh Madhavan for ensuring that I always had the resources and ability to do an excellent job; Dr. Roberto Benzo for his clinical expertise for my project; and finally Laura Menditto, who has been a great mentor during my Aventis fellowship at WVU, and also for her periodic phone calls during my dissertation to ensure that I was on the right track.

I would like to thank Nancy Atkins and Peggy King from the West Virginia Bureau of Medical Services, and Steve Small and Mike Madalena for providing WV Medicaid data. I sincerely wish to express my gratitude to Dr. Thomas Badgett from Kentucky's Department of Medicaid Services for his invaluable support and approval to use Kentucky’s Medicaid data for the project. The timely efforts of the EDS-KY Medicaid data analysts in transferring a large volume of data are most sincerely appreciated, especially Marilynn Hartmann, Bala Sivamohan, Mallika Eyunni, James Whiddon, Pamela Hershey, and Jeff Bortell. Also, many thanks to Gary Thompson for providing access to WV death records, and Bob Blackburn, George Robertson, Bill Miller, and Dr. Hacker for providing access to KY death records.

I would also like to acknowledge Dr. Gerald Hobbs for his statistical assistance, and the efforts of others at WVU for their support and encouragement, especially Dr. Scott, Mickey Howell, and Debbie Anderson. I am grateful for my fellow graduate students who have always been there for me during the good and the bad times in school, especially Murali, Vivek, Jay, Mariam, Reema, Khalid, and Ifti.

Finally, I owe all that I am today to my Ma, Daddy, sisters Sienna and Diana, and my love Rayan. They have never given up on me, and have always believed that I would make it. I also wish to thank A. Crystal and U. Mario for their constant love and encouragement, and Rayan’s family for their support and patience. 


\section{TABLE OF CONTENTS}

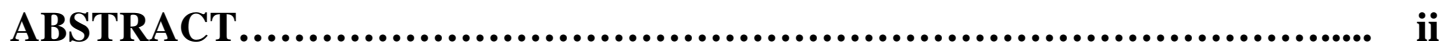

ACKNOWLEDGEMENTS............................................... iv

TABLE OF CONTENTS ................................................ v

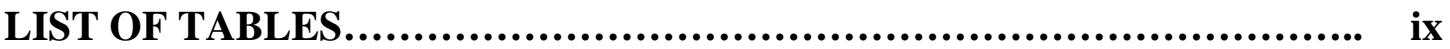

LIST OF FIGURES....................................................... xii

CHAPTER 1: INTRODUCTION AND STUDY OBJECTIVES................. 1

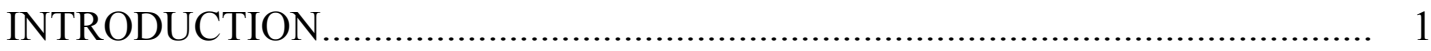

COPD: Prevalence, Costs, and Management................................. 1

Pharmacologic Options for Maintenance Therapy in COPD.................. 8

Summary of Efficacy and Effectiveness Evidence............................ 11

Pharmacoeconomic Evaluations in COPD ................................... 15

Rationale for Use of West Virginia and Kentucky Medicaid Claims Data....... 19

NEED FOR THE STUDY ............................................. 21

STUDY GOALS AND OBJECTIVES..................................... 23

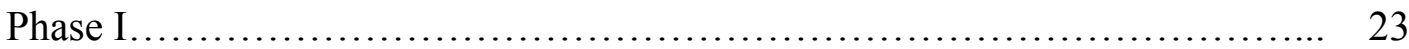

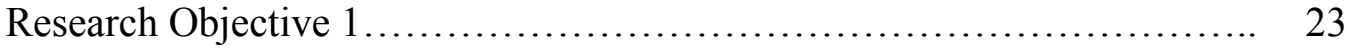

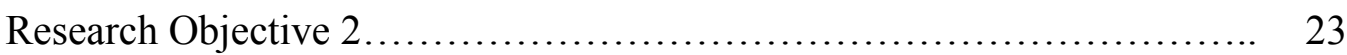

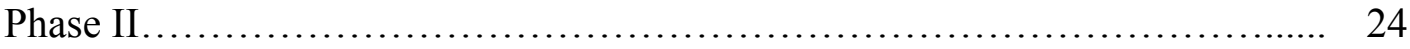

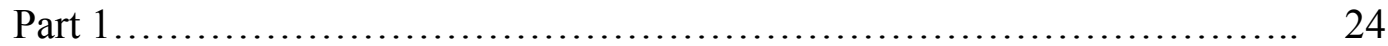

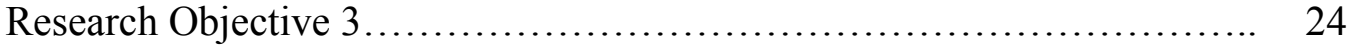

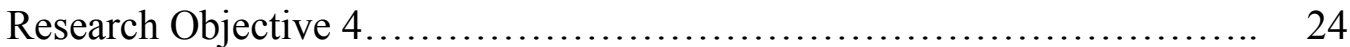

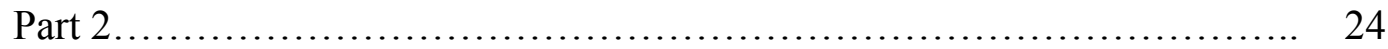

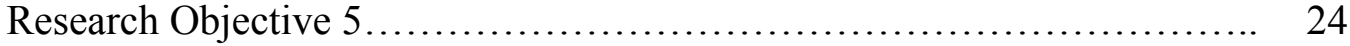

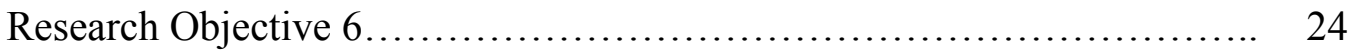

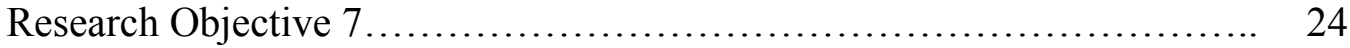

Phase III............................................................... 25

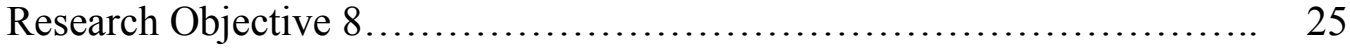




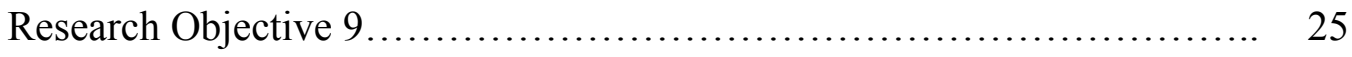

STUDY SIGNIFICANCE ................................................ 26

CHAPTER 2: LITERATURE REVIEW......................................... 27

INTRODUCTION TO THE DISEASE STATE-COPD ......................... 27

Pathogenesis, Pathophysiology, and Symptoms............................. 27

Risk Factors....................................................... 28

Diagnosis and Severity Staging.......................................... $\quad 30$

Epidemiology ........................................................ 31

PHARMACOTHERAPY FOR MAINTENANCE TREATMENT OF COPD...... 36

Medications for Maintenance Therapy of COPD ............................ 36

Patterns of Pharmacotherapy Use in COPD .................................. 40

EFFICACY AND EFFECTIVENESS OF MAINTENANCE

PHARMACOTHERAPY FOR COPD ....................................... 47

Outcomes to Assess Pharmacotherapy Effectiveness.......................... 47

Inhaled Bronchodilators: Long-acting Beta-agonists and Short-acting Anticholinergics.......................................................... 50

Inhaled Corticosteroids.................................................... 54

Combination of Inhaled Corticosteroid and a Long-acting Beta-agonist......... 65

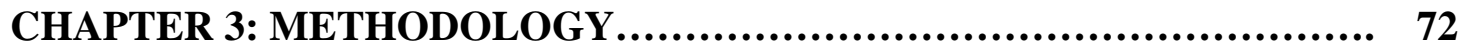

DATA DESCRIPTION AND VARIABLE EXTRACTION_................................... 72

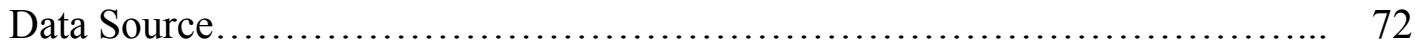

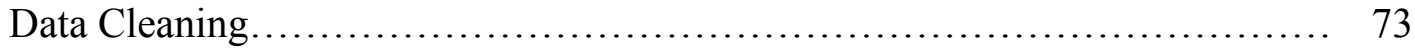

Measurement of Independent Variables................................... 76

Measurement of Outcome Variables....................................... 80

Analyses of Cost Data.............................................. 82

DESIGN AND ANALYSES OF STUDY GOALS AND OBJECTIVES.......... 84

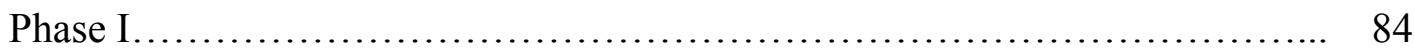

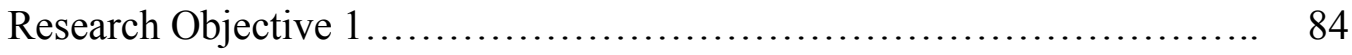




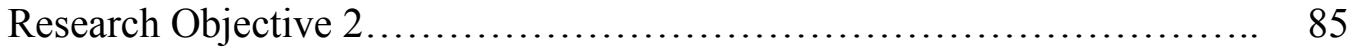

Phase II................................................................... 86

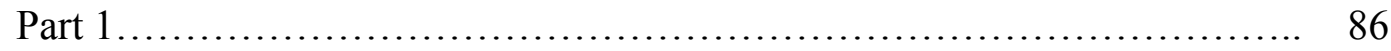

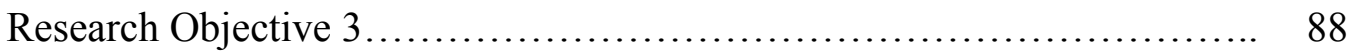

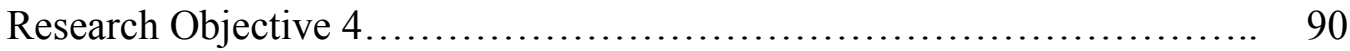

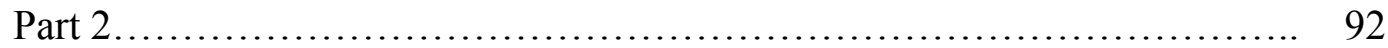

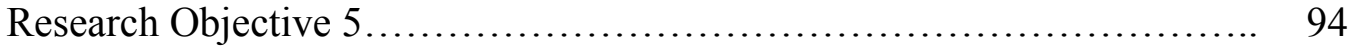

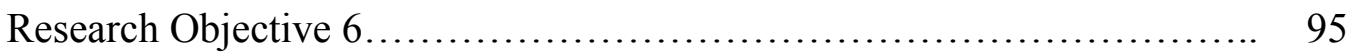

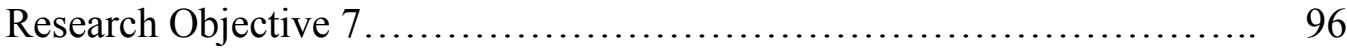

Phase III................................................................. 99

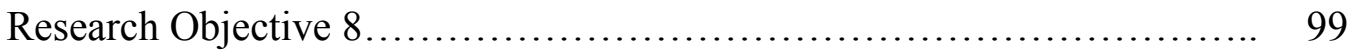

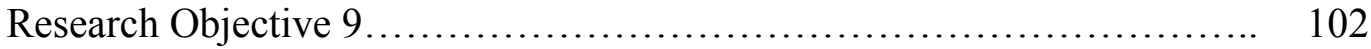

CHAPTER 4: RESULTS AND DISCUSSION............................. 107

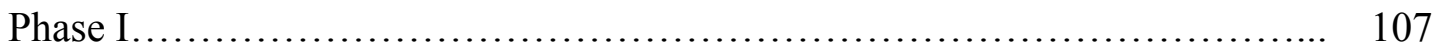

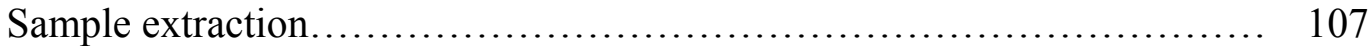

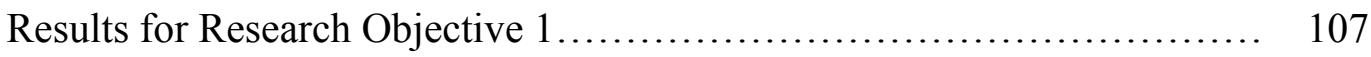

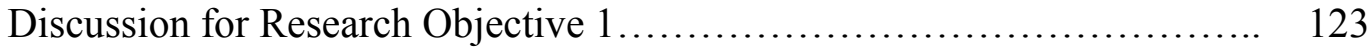

Results for Research Objective 2 ................................. 127

Discussion for Research Objective 2 ............................... 140

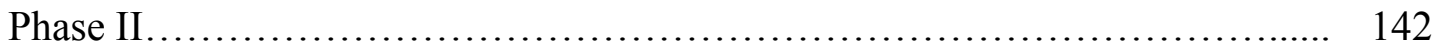

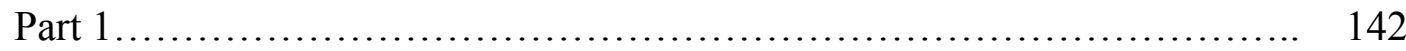

Sample extraction................................................ 142

Results for Research Objective 3.................................. 146

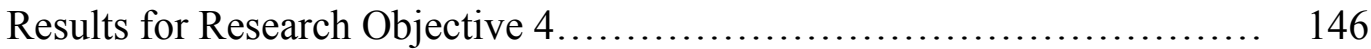

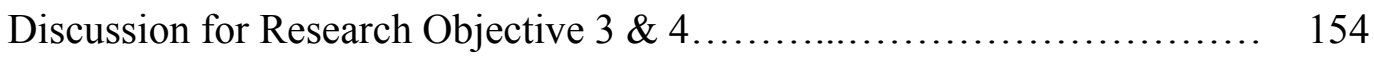

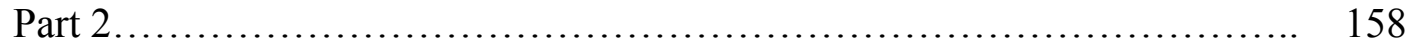

Sample extraction............................................... 158

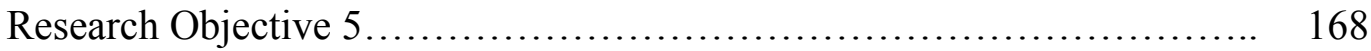




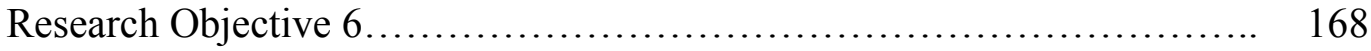

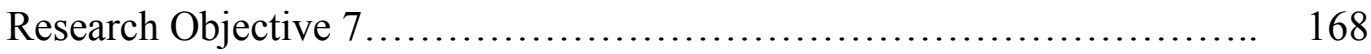

Discussion for Research Objective 5, 6, \& 7 .......................... 173

Phase III................................................................ 177

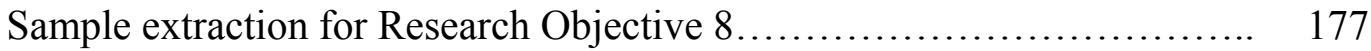

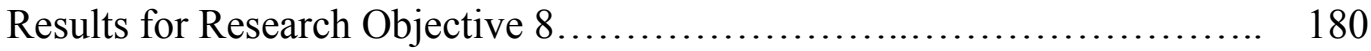

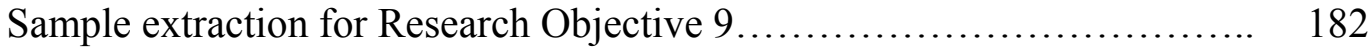

Results for Research Objective 9..................................... 185

Discussion for Research Objective 8 \& 9............................... 193

CHAPTER 5: SUMMARY AND CONCLUSIONS......................... 196

Summary of Results for Phase I............................................. 196

Summary of Results for Phase II........................................ 196

Summary of Results for Phase III........................................ 197

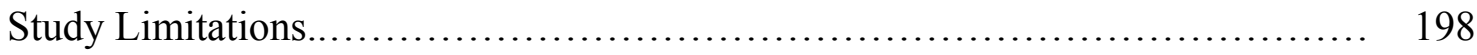

Conclusions and Implications of the Research................................ 200

Recommendations for Future Research.................................. 201

BIBLIOGRAPHY ........................................................... 203

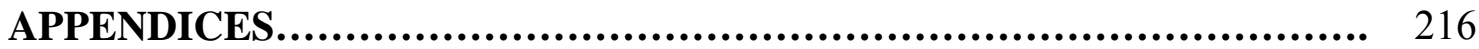

APPENDIX A: Regression Models for Analysis of Cost Data for Phase I 216 APPENDIX B: Logistic Regression Models for Obtaining Propensity Scores for Phase II - Part 2

CURRICULUM VITA..................................................... 247 


\section{LIST OF TABLES}

Table 1: Therapies for management of COPD .................................. 7

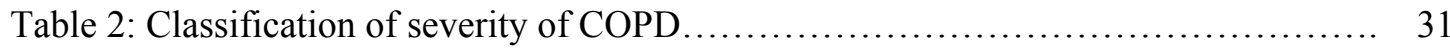

Table 3: Prevalence of chronic bronchitis and emphysema by demographic

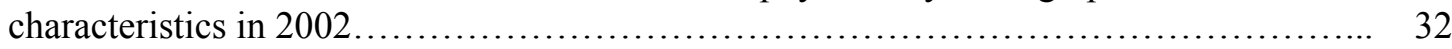

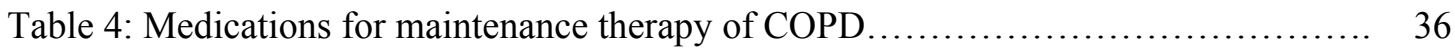

Table 5: Definition of exacerbations from observational studies of COPD .............. 49

Table 6: List of raw claims data files....................................... 73

Table 7: Scoring algorithm for D'Hoore-Charlson comorbidity index................. 78

Table 8: Sample extraction for Phase I........................................ 107

Table 9: Characteristics of recipients with and without COPD in WV and KY Medicaid... 109

Table 10: Total healthcare utilization and costs for recipients with and without COPD in

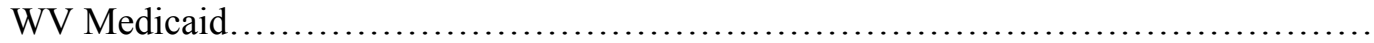

Table 11: Total healthcare utilization and costs for recipients with and without COPD in KY Medicaid......................................................................

Table 12: Mean incremental total healthcare costs incurred by recipients with COPD [Total healthcare costs incurred by recipients with COPD - Total healthcare costs incurred by recipients without COPD] in WV Medicaid.

Table 13: Mean incremental total healthcare costs incurred by recipients with COPD [Total healthcare costs incurred by recipients with COPD - Total healthcare costs incurred by recipients without COPD] in KY Medicaid.

Table 14: Total healthcare and COPD-related utilization and cost per recipient for recipients with $\operatorname{COPD}(\mathrm{N}=5,120)$ in WV Medicaid

Table 15: Total healthcare and COPD-related utilization and cost per recipient for recipients with $\operatorname{COPD}(\mathrm{N}=7,065)$ in $\mathrm{KY}$ Medicaid.

Table 16: Utilization of COPD-related prescription drugs and home oxygen therapy for recipients with COPD in WV $(\mathrm{N}=5,120)$ and $\mathrm{KY}(\mathrm{N}=7,065)$ Medicaid....

Table 17: Characteristics of recipients with COPD with and without concomitant asthma in WV and KY Medicaid.

Table 18: COPD-related utilization and costs per recipient for recipients with COPD with and without concomitant asthma in WV Medicaid.

Table 19: COPD-related utilization and costs per recipient for recipients with COPD with and without concomitant asthma in KY Medicaid.

Table 20: Utilization of COPD-related prescription drugs and home oxygen therapy for recipients with COPD with and without concomitant asthma in WV Medicaid. 
Table 21: Utilization of COPD-related prescription drugs and home oxygen therapy for recipients with COPD with and without concomitant asthma in KY Medicaid..............

Table 22: Mean incremental COPD-related cost per recipient for recipients with COPD with and without concomitant asthma in WV Medicaid...................................

Table 23: Mean incremental COPD-related cost per recipient for recipients with COPD with and without concomitant asthma in KY Medicaid...................................

Table 24: Sample extraction for Phase II - Part 1.

Table 25: Baseline characteristics of study sample by drug therapy status (IPR/ALB vs.

LABA: Phase II - Part 1).

Table 26: Exacerbations and costs in follow-up period by drug therapy status (IPR/ALB vs. LABA: Phase II - Part 1).

Table 27: Cox-Proportional hazards regression of the impact of drug therapy (IPR/ALB or LABA) on time to exacerbation.

Table 28: Logistic regression of the impact of drug therapy (IPR/ALB or LABA) on probability of exacerbation.

Table 29: Zero-inflated Poisson regression model of the impact of drug therapy

(IPR/ALB or LABA) on the frequency of exacerbations.

Table 30: Two part semi-logarithmic regression of the impact of drug therapy (IPR/ALB or LABA) on total COPD-related costs.

Table 31: Two part semi-logarithmic regression of the impact of drug therapy (IPR/ALB or LABA) on COPD-related prescription drug costs.

Table 32: Sample extraction for Phase II - Part 2.

Table 33: Baseline characteristics of study sample by drug therapy status

(ICS/LABA vs. SABD: Phase II-Part 2).

Table 34: Baseline characteristics of study sample by drug therapy status

(ICS vs. SABD: Phase II-Part 2)

Table 35: Baseline characteristics of study sample by drug therapy status (ICS/LABA vs. LABA: Phase II-Part 2).

Table 36: Risk of a severe exacerbation in the follow-up period by drug therapy status (Phase II-Part 2).

Table 37: Frequency of moderate exacerbations in follow-up period by drug therapy status (Phase II-Part 2).

Table 38: COPD-related costs in follow-up period by drug therapy status (Phase II-Part 2).

Table 39: Sample extraction for Phase III - Objective 8

Table 40: Characteristics of study sample by case-control status in pre-index period (Phase III-Objective 8).

Table 41: Exposure to inhaled corticosteroids in pre-index period by case-control status (Phase III - Objective 8) 
Table 42: Conditional logistic regression of the impact of inhaled corticosteroids on

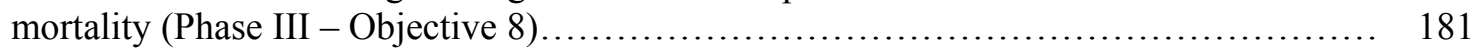

Table 43: Sample extraction for Phase III - Objective 9......................... 182

Table 44: Baseline characteristics of study sample by drug therapy status (ICS vs. no

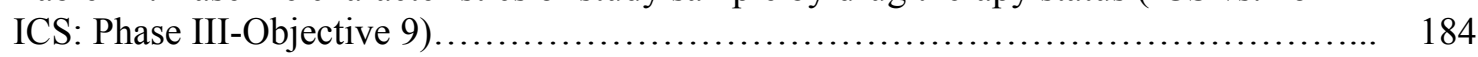

Table 45: Risk of mortality and severe exacerbation in follow-up period by drug therapy status (ICS vs. no ICS: Phase III - Objective 9) .......................................

Table 46: Logistic regression predicting the probability of being prescribed an inhaled corticosteroid

Table 47: Propensity score distribution by drug therapy status (ICS vs. no ICS: Phase III

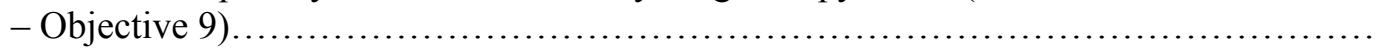

Table 48: Statistical tests of differences in characteristics within each propensity score quintile.

Table 49: Cox-Proportional hazards regression of the impact of drug therapy (ICS vs. no ICS) on time to death.

Table 50: Cox-Proportional hazards regression of the impact of drug therapy (ICS vs. no ICS) on time to severe exacerbation.... 


\section{LIST OF FIGURES}

Figure 1: GOLD treatment algorithm..................................... 8

Figure 2: Conceptual framework for Phase I................................. 23

Figure 3: Conceptual framework for Phase II................................ 25

Figure 4: Conceptual framework for Phase III................................. 25

Figure 5: COPD death rates in the United States.............................. 33

Figure 6: Identification of study sample for Part 1 of Phase II..................... 87

Figure 7: Historical cohort design to determine differences in time to first exacerbation (Phase II - Part 1)....................................................... 89

Figure 8: Historical cohort design to determine differences in number of exacerbations (Phase II - Part 1) ........................................................... 89

Figure 9: Historical cohort design to determine differences in COPD-related costs (Phase II - Part 1) .......................................................... 90

Figure 10: Identification of study sample for Part 2 of Phase II.................... 92

Figure 11: Historical cohort design to determine differences in time to first severe exacerbation (Phase II - Part 2).

Figure 12: Historical cohort design to determine differences in number of moderate exacerbations (Phase II - Part 2).

Figure 13: Historical cohort design to determine differences in COPD-related costs (Phase II - Part 2)

Figure 14: Identification of study sample for research objective 8 of Phase III......... 100

Figure 15: Case-control design to determine impact of ICS on mortality............... 101

Figure 16: Identification of study sample for research objective 9 of Phase III........... 102

Figure 17: Historical cohort design to determine differences in mortality............... 104

Figure 18: Adjusted Kaplan-Meier survival curve of time to exacerbation by drug therapy group (IPR/ALB vs. LABA: Phase II - Part 1)

Figure 19: Adjusted Kaplan-Meier survival curve of time to death by drug therapy group (ICS vs. no ICS: Phase III - Objective 9).

Figure 20: Adjusted Kaplan-Meier survival curve of time to severe exacerbation by drug therapy group (ICS vs. no ICS: Phase III - Objective 9) 


\section{ChAPTER 1: InTRODUCTION AND STUdy OBJECTIVES}

\section{INTRODUCTION}

The impact of smoking has been most prominent in Chronic Obstructive Pulmonary Disease (COPD) relative to other diseases (Pauwels, Buist, Calverley, Jenkins, \& Hurd, 2001). Although it has been estimated that 15 percent of smokers eventually develop COPD, the attributable risk fraction due to smoking is very high with smoking being responsible for 80 percent to 90 percent of COPD cases (Sin, McAlister, Man, \& Anthonisen, 2003c). The

picture gets even grimmer with recent evidence from one study in Sweden showing that up to 50 percent will progress to develop COPD (Lundback et al., 2003). Thus, it is not surprising that smoking cessation is the only treatment modality in COPD that affects the progression and mortality of the disease as was evidenced from a large cohort in the Lung Health Study (LHS) (Anthonisen et al., 1994). The study showed that smoking cessation led to a 50 percent reduction in the rate of lung function decline (Anthonisen, Connett, \& Murray, 2002). These statistics should be comforting knowing that a preventable and treatable solution is available for COPD. However, availability does not imply attainability. Data from the LHS also showed that after 11 years, only 22 percent of those in a smoking cessation program stopped smoking, whereas only 6 percent not in a program stopped smoking (Murray et al., 2000). Given these data, should we still be concerned about COPD as a public health problem? The answer is yes, and lies in an understanding of what constitutes COPD and its consequences on morbidity, mortality, and healthcare costs. Chapter one will provide a brief description of the COPD disease state and pharmacologic options used for maintenance therapy that provide an understanding for the need for the proposed study, and the study goals and objectives.

\section{COPD: Prevalence, Costs, and Management}

COPD is defined as a clinical syndrome that refers to the presence of two distinct pathoclinical entities: chronic bronchitis and emphysema (Mapel, 2004). The definition of COPD excludes other obstructive pulmonary diseases that are associated with poorly reversible airflow limitation such as bronchiectasis, cystic fibrosis, fibrosis due to tuberculosis, and asthma, except if these conditions overlap with COPD. COPD is defined as 
a syndrome characterized by progressive airflow limitation that is not fully reversible, and is caused by an abnormal inflammatory reaction to the chronic inhalation of particles, primarily tobacco smoke (Pauwels et al., 2001). Airflow limitation is the slowing of expiratory airflow as measured by spirometry (Mannino, 2003). Spirometric measures of airflow limitation include forced expiratory volume in one second ( $\left.\mathrm{FEV}_{1}\right)$ and forced vital capacity (FVC), which is the maximum volume of air in liters that can be forcibly and rapidly exhaled in one second or full exhalation, respectively. In patients with $\mathrm{COPD}$, the $\mathrm{FEV}_{1}$ and $\mathrm{FVC}$ are reduced relative to normal predicted values. Airflow limitation can be reversible or irreversible depending on the response after bronchodilator administration. Based on the Global Initiative for Obstructive Lung Disease (GOLD) and American Thoracic Society (ATS) definitions, an $\mathrm{FEV}_{1}$ increase of 12 percent $(200 \mathrm{ml})$ above baseline after administration of inhaled bronchodilators or corticosteroids is considered to be a demonstration of reversibility. Originally considered to be a disease with irreversible airways obstruction (i.e. no response to bronchodilators), recent studies estimate 23 percent to 42 percent of patients with COPD exhibit bronchodilator reversibility depending on the criteria used (Calverley, Burge, Spencer, Anderson, \& Jones, 2003). Therefore, airflow limitation in COPD is characterized as not fully reversible rather than the old myth of irreversible airways obstruction. The progressive nature of the disease refers to the accelerated decline in lung function as measured using $\mathrm{FEV}_{1}$, from the normal rate in adults over 30 years of age of approximately $30 \mathrm{ml}$ per year to nearly $60 \mathrm{ml}$ per year (Anthonisen et al., 2002).

The available prevalence data of COPD are considered to under-estimate the total burden of disease, primarily because the disease is usually diagnosed after moderate advancement has occurred and clinical symptoms become apparent (Pauwels et al., 2001). In 2002, the National Health Interview Survey (NHIS) in the U.S, estimated that 11.2 million ( $5 \%)$ people over 17 years of age reported a physician diagnosis of COPD. Of these, 9.1 million reported chronic bronchitis and 3.1 million reported emphysema (American Lung Association, 2004) $)^{1}$. Evidence of under-diagnosis of the condition is seen from prevalence information objectively obtained using spirometry from the 1994 National Health and

\footnotetext{
${ }^{1}$ COPD totals do not take into account the overlap of persons with both diseases. Therefore, COPD totals will be lower than the sum of chronic bronchitis and emphysema estimates.
} 
Nutrition Examination Surveys (NHANES). In this study, 24 million people over 25 years of age had COPD, more than twice that of the 2002 NHIS estimate that used physician diagnosis as a criterion.

The decline in lung function in COPD impacts mortality due to the development of several fatal conditions such as respiratory failure, cor pulmonale, and pulmonary embolism. Mortality in COPD is further compounded by the fact that a majority of deaths are attributed to other causes, primarily cardiovascular. In one study, approximately 45 percent of decedents with COPD mentioned on their death certificates have COPD listed as an underlying cause of death (Mannino, Brown, \& Giovino, 1997). In the U.S., COPD and allied conditions (includes asthma and bronchiectasis) were the fourth leading cause of death in 2002 (NHLBI, 2004). On a global level COPD was ranked as the sixth leading cause of death in 1990, and is projected to rise to third by 2020 (Murray \& Lopez, 1997).

\section{$\underline{\text { Costs of COPD }}$}

A highly prevalent and fatal disease, COPD also exerts substantial economic consequences to the U.S. healthcare system. In 2004, the National Heart, Lung and Blood Institute (NHLBI) estimated that COPD cost the U.S. healthcare system a total of $\$ 37.2$ billion (NHLBI, 2004). The direct costs totaled $\$ 20.9$ billion and the indirect cost due to morbidity and mortality totaled $\$ 7.4$ billion and $\$ 8.9$ billion, respectively. This section will review COPD cost-of-illness studies in the U.S. to identify components of care that are major cost drivers, and factors affecting costs. This information is useful for developing interventions to target high-risk, high-cost sub-groups.

Two studies using a composite of national databases in the U.S. assessed costs related to COPD, and reached different conclusions (Wilson, Devine, \& So, 2000; Ward, Javitz, Smith, $\&$ Bakst, 2000). Wilson et al. found hospitalizations (57\%) and medications (40\%) to be the first and second highest cost categories (the cost of oxygen therapy was not estimated) (Wilson et al., 2000). On the other hand, Ward et al. found oxygen therapy (35\%) and hospitalizations (24\%) to be the first and second highest cost categories (Ward et al., 2000). COPD-related medications comprised only $7 \%$ of total costs in study by Ward et al. as determined from data of the National Medical Expenditure Survey 1987 (NMES) and the National Ambulatory Medical Care Survey 1990 (NAMCS), as opposed to the estimate of Wilson et al. that was based on expert opinion of prescription drug patterns. Strassels et al. 
analyzed the 1987 NMES only, and also found that all prescription drugs used by patients with COPD (COPD-related and non-COPD related) accounted for 8 percent of total costs, while hospitalizations were 70 percent of total costs (Strassels, Smith, Sullivan, \& Mahajan, 2001).

Wilson et al. and Ward et al. assessed the cost of only COPD-related care; however Strassels et al. additionally assessed the cost of non-COPD related care. Thus, average cost per patient with COPD for Wilson et al. was \$896 (1996 USD) while Strassels et al. found an average of $\$ 6,469$ (1987 USD) per patient with COPD of which only 25 percent was related to COPD. Another study found that pulmonary-related outpatient utilization, drugs, and oxygen therapy accounted for only 25 percent of the difference in total outpatient and pharmacy utilization between COPD and non-COPD patients (Mapel et al., 2000a).

Similarly, analysis of managed care data showed that 23 percent of total monthly health care costs were related to COPD (Kesten S, 2001). These findings indicate that although COPD itself can increase costs for patients, the presence of smoking-related comorbidities (e.g. coronary heart disease, congestive heart failure, hypertension, malignant disease, gastritis) have an enormous influence on total costs incurred by a patient with COPD (Mapel et al., 2000a). Thus, it is important to include costs of non-COPD related care to permit accurate estimation of costs incurred by patients with COPD.

Analyses that compare total costs (COPD and non-COPD related) between COPD and non-COPD patients provide estimates of the cost difference between these subsets, and allow one to determine the magnitude of the amount that is incurred due to the presence of COPD. Mapel et al. found that total health care costs in patients with COPD were twice those of nonCOPD patients with an incremental cost of \$6,039 per case (Mapel et al., 2000a). These differences were evident in all components of health care (inpatient, outpatient, and pharmacy services), with the largest difference for inpatient services. Similar to findings by Strassels et al., Grasso et al. also found that per capita expenditures for Medicare beneficiaries with COPD were 2.4 times those of average Medicare beneficiaries (Grasso, Weller, Shaffer, Diette, \& Anderson, 1998). Most studies have found a ratio of 2:1 when comparing COPD and non-COPD patients except one where total monthly costs per patient were six times higher than non-COPD patients (Kesten S, 2001). 
The analysis by Strassels et al. (Strassels et al., 2001) noted that approximately 20 percent of patients with COPD account for about 74 percent of total expenditures, while Grasso et al. (Grasso et al., 1998) found that 10 percent of patients accounted for nearly half of total expenditures. This is probably a reflection of the level of disease severity and higher number of co-morbidities of this subset of the COPD population. The impact of disease severity level on costs has been assessed in two studies. Using disease severity based on $\mathrm{FEV}_{1}$ according to ATS criteria, one study found that annual median costs per patient for severe COPD (stage III) were $\$ 10,812$, more than twice that of patients with moderate COPD (stage II), \$5,037 (Hilleman, Dewan, Malesker, \& Friedman, 2000). Similarly, costs of patients with moderate COPD were nearly three times greater than costs of patients with mild COPD. As expected, hospitalizations accounted for the largest proportion of total costs regardless of disease severity, whereas oxygen therapy accounted for almost 15 percent to 20 percent of total costs only for moderate and severe patients, respectively. The other study assessed disease severity from patient's perceptions or scores on the Medical Research Council Dyspnea Scale (MRC) (Halpern, Stanford, \& Borker, 2003). Patients with severe COPD, as measured by either the MRC scale or patient's perceptions, incurred almost three to six times higher costs than mild or moderate patients with COPD. Similar to disease severity, decrease in self-reported health status is related to increase in costs of COPD (Strassels et al., 2001).

Age does not seem to influence the total costs incurred by patients with COPD, with costs being slightly higher in those over 64 years compared to those younger than 65 years (Strassels et al., 2001; Mapel et al., 2000a). Conflicting evidence exists for variation in total COPD costs by physician specialty. One study found no significant differences in charges for care rendered by pulmonologists, general internists, or family physicians (Strauss, Conrad, LoGerfo, Hudson, \& Bergner, 1986). On the other hand, Hilleman et al. found care provided by pulmonologists to be higher than that provided by primary care physicians $(\$ 4,026$ vs. $\$ 7,805$ ) (Hilleman et al., 2000). Interestingly enough though, patients with moderate and severe COPD treated by pulmonologists ( $\$ 4,439$ and $\$ 10,226$, respectively) had significantly lower total costs than those treated by primary care physicians $(\$ 5,270$ and $\$ 11,105$, respectively). 
Co-morbidities, particularly cardiovascular-related causes, substantially increase the costs for patients with COPD as seen in the evidence above. According to recent estimates, patients with COPD with a concomitant diagnosis of asthma constitute almost half of all patients with COPD (Mapel et al., 2000b; Mannino, 2003). Asthma and COPD are both obstructive lung diseases with airflow limitation. The difference however, is that in asthma; the response to bronchodilator administration is primarily reversible while in COPD it is partially reversible or irreversible. Thus, patients with concomitant asthma and COPD probably represent a sub-group of patients with COPD with more reversibility relative to patients with COPD without asthma. This however, has not been documented as yet. The impact of this reversibility on prognosis and response to treatment is of increasing interest in COPD currently, and recent evidence suggests that degree of bronchodilator reversibility may not impact these factors (Tashkin \& Kesten, 2003).

Only two studies have provided some information regarding the impact of a concomitant diagnosis of asthma in patients with COPD. Data from the 1996 NHIS survey showed that participants reporting both COPD and asthma had more days of restricted activity (62.5 vs. 31.0 days), bed confinement ( 31.5 vs. 13.1 days), and work loss ( 8.2 vs. 2.4 days) than did participants with COPD alone (Mannino, 2002). The increased use of resources is also reflected in a Canadian study that determined spirometry and drug use in COPD and asthma patients (Anthonisen, Woodlrage, \& Manfreda, 2005). Patients with COPD and asthma had considerably higher rates of drug use and slightly higher spirometry rates than patients with COPD-only. The rate of use of inhaled corticosteroids was similar to the rate of use in patients with only asthma, and that of inhaled anti-cholinergics was similar to that in COPDonly patients. These data suggest that a concomitant diagnosis of asthma in patients with COPD may identify a high-cost group. However, none of the cost-of-illness studies have stratified based on presence of asthma. Such studies are needed so that appropriate targets for disease management can be determined.

\section{Management of COPD}

The enormous clinical and economic impact of COPD has prompted the development of the Global Initiative for Chronic Obstructive Pulmonary Disease (GOLD) in 1997. The specific aim of GOLD is to improve the prevention and management of COPD by providing the best available evidence on effectiveness of therapeutic options and validated current 
concepts of COPD pathogenesis (Pauwels et al., 2001). This committee has adequately outlined the goals of COPD management that include reduction of disease progression and mortality, relief of symptoms, improvement in exercise tolerance and health status, and prevention of exacerbations and complications. These goals should be accomplished by a management plan which includes four components:

1. Diagnosis and monitoring of the disease

2. Reduction of risk factors

3. Management of stable COPD

4. Management of exacerbations

There are several therapies available, both pharmacologic and non-pharmacologic, that form aspects of the above four-component plan (Table 1). Management consists of a stepwise increase in treatment depending on the severity of the disease. Once symptom control has been achieved, reduction of therapy is usually not possible because of the progressive nature of the disease. Some therapies are applicable to all patients with COPD such as smoking cessation, immunizations, and bronchodilators. However, not all therapies need to be used simultaneously in all patients with COPD. When multiple therapies are used in combination, they should be incorporated in a pre-determined plan so as to assist integration in a coordinated manner. Since the proposed study will evaluate the effectiveness and costs of pharmacotherapy, further sections will focus on the role of pharmacotherapy in COPD.

\title{
TABLE 1: Therapies for management of COPD
}

\author{
Smoking cessation \\ Immunizations: Influenza and Pneumococcal \\ Pharmacotherapy: Bronchodilators, Inhaled and oral corticosteroids, antibiotics \\ Oxygen \\ Pulmonary Rehabilitation \\ Mechanical ventilation \\ Surgery: Lung volume reduction, lung transplantation
}




\section{Pharmacologic Options for Maintenance Therapy in COPD}

Two components of the COPD management plan refer to management of a stable state and an exacerbated state. The stable state refers to the presence of all or few symptoms such as cough, sputum production, wheezing and dyspnea without the need for additional treatment or management. A patient with COPD also experiences exacerbations which are defined as episodes with increase or worsening of symptoms relative to the day-to-day fluctuations of the stable state that may require additional medical treatment (Burge \& Wedzicha, 2003). The relevant question then would be "is pharmacotherapy required only when symptoms exacerbate, or is there a need for maintenance therapy for the stable state?" Evidence from controlled clinical trials of drugs suggests that maintenance pharmacotherapy is indeed beneficial. Benefits of this approach include reduction in the frequency or severity of exacerbations, and improvement in symptom scores, exercise tolerance and overall healthrelated quality-of-life (HRQol) (Tashkin \& Cooper, 2004; Sin et al., 2003c).

Two broad categories of drugs are used for maintenance therapy of COPD: bronchodilators and inhaled corticosteroids (ICS). Bronchodilators include drugs from three distinct classes: beta-agonists, anti-cholinergics, and methylxanthines. Beta-agonists and anti-cholinergics are considered treatments of choice, and include short-acting and longacting formulations. Methylxanthines are infrequently used due to numerous side effects, and are currently used only as additional therapy for advanced disease and those with less than optimal control with inhaled bronchodilators.

\section{FIGURE 1: GOLD treatment algorithm}

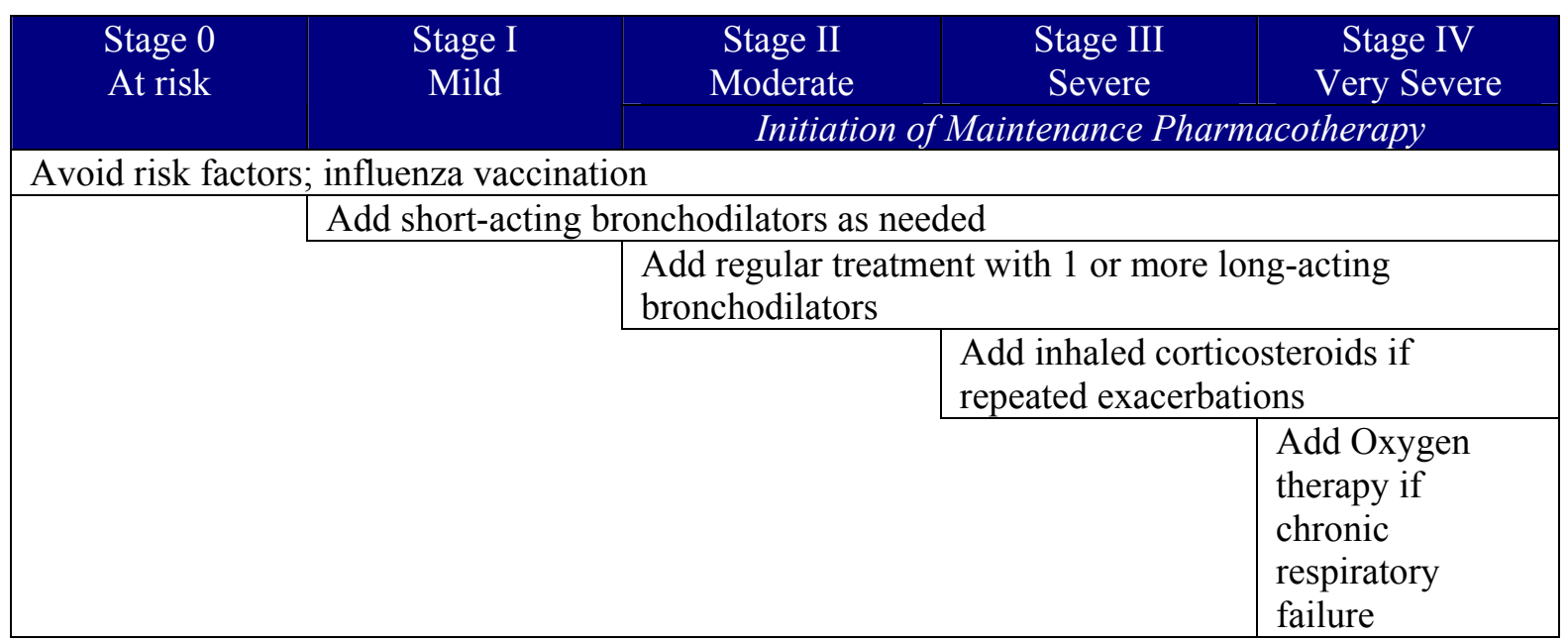

Adapted from Reference (GOLD, 2004) 
The therapeutic algorithm outlined by the GOLD initiative (figure 1) depicts a stepwise increase in pharmacologic therapy as disease severity increases, based on $\mathrm{FEV}_{1}$ level (GOLD, 2004). Patients with stage 1 (mild COPD) are defined as those with few or intermittent symptoms. Thus, pharmacotherapy for mild COPD is on an as-needed basis, generally using short-acting bronchodilators such as albuterol. At this point it should be noted that pharmacotherapy for mild COPD is on an as-needed basis, while maintenance pharmacotherapy is relevant only for moderate-to-severe COPD.

Short-acting bronchodilators are to be used as rescue medication as-needed at all stages. Among short-acting bronchodilators, current recommendations are that use of short-acting beta-agonists alone should not be considered for maintenance therapy, and are to be used only as rescue medication. On the other hand, the short-acting anti-cholinergic, ipratropium, has been the drug of choice for maintenance therapy. Although it can be used on an asneeded basis, its slower onset of action compared to short-acting beta-agonists, does not make it suitable for this purpose. Considered to be equivalent to ipratropium, a combination of ipratropium (short-acting anti-cholinergic) and albuterol (short-acting beta-agonist) occupies an important place in maintenance therapy for COPD (Petty, 1995; Rennard, 1995).

Regular treatment with a long-acting bronchodilator is now recommended for patients with stage II (moderate COPD) disease because of reasons of efficacy and convenience. Additionally, a combination of long-acting bronchodilators from two different classes (betaagonists and anti-cholinergics) is increasingly being considered, following the extremely effective combination of two short-acting bronchodilators from these two classes (Tennant, Erin, Barnes, \& Hansel, 2003). However, no clinical studies are available currently regarding the efficacy of combination of long-acting bronchodilators. For combination therapy, a single drug may be administered or a combination product may be used. The two short-acting bronchodilators, albuterol (beta-agonist) and ipratropium (anti-cholinergic) are available in a combination product. Oral theophylline may also be considered for stage II patients, although this option is usually less preferred. As is evident in figure 1, the use of bronchodilators is recommended for all disease severity stages. However, as disease severity increases to stage III (severe) and stage IV (very severe), addition of inhaled corticosteroids (ICS) is usually recommended. These drugs are also recommended for those with stage II with frequent exacerbations. 
The present study will evaluate outcomes of maintenance pharmacotherapy in COPD, and will be discussed below. First, a discussion of relevant outcome measures in COPD is important before presenting evidence of the efficacy and effectiveness of pharmacotherapy.

\section{Outcome Measures in COPD Pharmacotherapy}

$\mathrm{FEV}_{1}$ is an important clinical measure in COPD that indicates the degree of airflow limitation, a primary characteristic of COPD (Pauwels et al., 2001). However, the use of $\mathrm{FEV}_{1}$ as a sole outcome measure to evaluate treatment effectiveness in COPD has its limitations due to several reasons. First, COPD is a complex multisystem disorder that affects not only the lungs but also other organs. In addition, the pathophysiological processes that occur in the lungs are not captured entirely by $\mathrm{FEV}_{1}$, such as lung hyperinflation leading to increases in lung volumes and abnormal gas exchange at the alveolar level (Briggs, Jr., 2004). The problem is compounded because of the difficulty in measuring lung volumes in a reproducible manner to guide therapy decisions. In fact, the sensation of breathlessness (dyspnea), and exercise tolerance are better indicators of changes in lung volumes (O'Donnell, 2000). Symptom scores, exacerbations, and HRQol can be described as more summative measures that capture physical and psychosocial aspects of this disease (Jones, 2001). The inadequacy of $\mathrm{FEV}_{1}$ as an outcome measure is also seen in the poor correlation of this measure with symptom score, HRQol, and dyspnea (O'Donnell, 2000; Jones, 1995). Among the outcome measures, exacerbations are the most highly and positively correlated with $\mathrm{FEV}_{1}$. However, there are still about 20 percent of patients with moderate-to-severe disease (lower $\mathrm{FEV}_{1}$ ) that do not experience any exacerbations (Burge et al., 2000).

Second, bronchodilators are considered standard drug therapy in COPD, and their primary mode of action is airway smooth muscle relaxation (Lipson, 2004). $\mathrm{FEV}_{1}$ is an incomplete surrogate clinical marker of this outcome, and represents only a portion of the complex clinical consequences of COPD (Jones \& Kaplan, 2003a; O'Donnell, 2000). Additionally, drugs can induce changes in other areas such as improvement in dyspnea and symptoms through other mechanisms. Therefore, evaluation of treatment options in COPD cannot solely be restricted to the improvement in $\mathrm{FEV}_{1}$.

Third, since COPD is described as a disease that is partially reversible (a low FEV that responds partially or not at all to bronchodilators or steroids), it seems almost paradoxical to 
use $\mathrm{FEV}_{1}$ as a sole outcome measure on which to base the effectiveness of therapy (Celli, 2000).

This multifaceted nature of COPD has led to the use of other outcome measures to evaluate the effectiveness of therapy in COPD. Examples of other outcome measures to evaluate the effectiveness of COPD therapies include, but are not restricted to; improvements in dyspnea, exercise tolerance, symptoms (exacerbations) and HRQoL (Gross, 2003). The tangible nature of these outcomes to the patient as well as their clinical relevance due to their prognostic characteristics provide adequate justification for use of these outcome measures (Fan, Curtis, Tu, McDonell, \& Fihn, 2002; Nishimura, Izumi, Tsukino, \& Oga, 2002). Moreover, there is conflicting evidence that drugs in COPD impact disease progression as measured by $\mathrm{FEV}_{1}$ and mortality. Therefore, other outcome measures assume greater importance in evaluating COPD pharmacotherapy.

\section{Summary of Efficacy and Effectiveness Evidence}

$\underline{\text { Inhaled Broncodilators: Long-acting beta-agonists and Short-acting anticholinergics }}$

Desirable features of long-acting beta-agonists, salmeterol and formoterol, such as the extended duration of bronchodilation and twice daily administration have increased the popularity of these agents for maintenance therapy in COPD. A recent update to the GOLD

guidelines recommends that long-acting beta-agonists are more effective and convenient than the short-acting anti-cholinergic, ipratropium (GOLD, 2004). However, data from four comparative trials provide inconsistent results regarding the impact of long-acting betaagonists with ipratropium, particularly for exacerbations. A recent review of these trials using a best evidence synthesis approach instead of a meta-analysis, concluded that longacting beta-agonists were comparable to ipratropium in terms of lung function as measured by $\mathrm{FEV}_{1}$ and dyspnea reduction (Husereau, Shukla, Boucher, Mensinkai, \& Dales, 2004). Salmeterol showed inconsistent results, with one trial showing a significantly longer time to an exacerbation relative to ipratropium (Mahler et al., 1999), while another trial showed no differences in the proportion of patients with exacerbations requiring oral corticosteroids (Rennard et al., 2001). Only one of two formoterol trials assessed exacerbation rate, and found the long-acting beta-agonist to reduce the percentage of bad days (mild exacerbations) relative to ipratropium, but have no impact on moderate or severe exacerbations (Dahl et al., 
2001). The inconsistency of the effect of long-acting beta-agonists on exacerbation rate is also seen in comparison to placebo. Sin et al. conducted a meta-analysis from eight randomized trials of at least 3 months of the impact of long-acting beta-agonists and found a 21 percent significant reduction compared to placebo on rate of exacerbations (Sin et al., 2003c). In contrast, a Cochrane review found an insignificant 31 percent risk reduction compared to placebo (Nannini, Lasserson, \& Poole, 2003). Another review has attributed the lack of an effect to the duration of trials (Tashkin et al., 2004). Most of the trials were conducted for a maximum of 12 weeks.

Observational studies have assessed the impact of long-acting beta-agonists on exacerbations over a period of one year. Only severe exacerbations have been evaluated in these studies. Two studies used a similar cohort design with administrative claims databases, and assessed the risk of an exacerbation requiring hospitalization after one year following initiation of therapy (Burney et al., 2003; Rascati, Stanford, \& Borker, 2005). A trend towards a reduced risk was seen for long-acting beta-agonists compared to ipratropium that was not statistically significant in both studies. Study design aspects such as inadequate control of disease severity, and lack of assessment of other outcomes such as moderate exacerbations and costs suggest the need for additional studies to establish the value of longacting beta-agonists in COPD relative to ipratropium.

$\underline{\text { Inhaled corticosteroids (ICS) }}$

As disease severity increases, the GOLD guidelines recommend addition of ICS for the treatment of COPD (Pauwels et al., 2001). There is much controversy regarding the use of ICS for COPD (Calverley, 2000; Barnes, 2000a). Although, considered as first-line therapy in asthma, differences in inflammatory processes between asthma and COPD do not adequately support the use of these drugs for COPD (Barnes, 2000b). There was major interest in determining if ICS could reduce the progression of COPD given their effectiveness in asthma. Six randomized controlled trials with duration of at least 2 years showed no impact on the rate of decline in lung function (Vestbo et al., 1999; Pauwels et al., 1999; Burge et al., 2000; Lung Health Study Group, 2000; Renkema, Schouten, Koeter, \& Postma, 1996; Weir, Bale, Bright, \& Sherwood, 1999). However, two meta-analyses that pooled data from these trials reached opposite conclusions. The first meta-analysis found a statistically and clinically insignificant difference of $+5.31 \mathrm{ml} /$ year in the rate of $\mathrm{FEV}_{1}$ 
decline (Highland, Strange, \& Heffner, 2003), whereas the second meta-analysis found a statistically significant difference of $7.7 \mathrm{ml} /$ year (Sutherland, Allmers, Ayas, Venn, \& Martin, 2003). The clinical significance of the estimate from the latter study is questionable because of the small magnitude.

The lack of an effect on disease progression leads one to conclude that ICS may not impact mortality. This has been shown in all clinical trials and meta-analyses, but not observational studies. Six observational studies have found an effect on decreasing mortality by 25 percent to 75 percent (Sin \& Man, 2003a; Sin et al., 2003a; Soriano, Kiri, Pride, \& Vestbo, 2003; Soriano et al., 2002; Burney et al., 2003). Two observational studies however, mirror clinical trial results (Suissa, 2003; Fan et al., 2003). Some researchers have attempted to explain the 25 percent to 75 percent reduction in mortality found in observational studies as evidence that ICS may actually modify the progression of the disease based on the significant reduction in lung function decline found in the meta-analysis described above (Burge \& Lewis, 2003). It is important to explain reductions of such magnitude from observational studies given the totality of evidence that ICS do not modify the progression of the disease. One possible hypothesis is that ICS are able reduce mortality by reducing the rate or severity of exacerbations. Exacerbations are known to have a significant impact on mortality (Groenewegen, Schols, \& Wouters, 2003), and thus the effect of ICS on mortality may be mediated through its impact on exacerbations.

Of six randomized trials that reported ICS use and exacerbations, two showed statistically significant differences (Burge et al., 2000; Paggiaro et al., 1998) while four did not show any significant difference (Vestbo et al., 1999; Bourbeau, Rouleau, \& Boucher, 1998; Weir et al., 1999; Lung Health Study Group, 2000). A meta-analysis that pooled data from these studies showed that ICS reduced the risk of exacerbations by 30 percent $(\mathrm{RR}=0.70 ; 95 \% \mathrm{CI}$ : 0.58 to 0.84) (Alsaeedi, Sin, \& McAlister, 2002). The beneficial impact of ICS on exacerbations needs to be described beyond summary estimates. Analyses from the two clinical trials that found a beneficial effect of ICS on exacerbations have found no impact on the total proportion of patients using ICS who experienced at least one exacerbation (Burge et al., 2000; Paggiaro et al., 1998). However, there was a significant difference in the proportion of patients experiencing moderate to severe exacerbations. Thus, ICS may significantly impact the severity of the exacerbation but not the overall frequency. 
The inconsistency of the effect of ICS on exacerbations has been seen in observational studies for severe but not moderate exacerbations. Five observational studies found that ICS significantly reduced the risk for exacerbation requiring hospitalization ( $\operatorname{Sin} \& \mathrm{Tu}, 2001$; $\operatorname{Sin}$ et al., 2003a; Soriano et al., 2003; Burney et al., 2003; Rascati et al., 2005), and two observational studies found no significant effect (Fan et al., 2003; Bourbeau, Ernst, Cockcoft, \& Suissa, 2003). Two clinical trials have shown that the risk for moderate exacerbations is also reduced (Burge et al., 2000; Paggiaro et al., 1998). However, in only two observational studies that have assessed this outcome, no significant effects were found (Fan et al., 2003; de Melo, Ernst, \& Suissa, 2004a). On the contrary, a trend for increased risk of moderate exacerbations was noted (de Melo et al., 2004a).

Combination of inhaled corticosteroids and long-acting beta-agonists

Combination therapies are gaining importance in COPD due to the chronic and progressive nature of COPD, as well as complementary mechanisms of actions of drugs from different classes. The combination of ICS and long-acting beta-agonists are increasingly being considered as an important combination as these two classes of drugs have complementary effects on each other's mode of action, providing additive or synergistic effects (Sin, Johnson, Gan, \& Man, 2004). Unlike the conflicting evidence regarding the use of ICS on exacerbations, the use of the combination of ICS and long-acting beta-agonist have shown a reduction in exacerbation rates in most clinical trials, meta-analyses, and observational studies.

At least three randomized trials have assessed the impact of the fluticasone/salmeterol combination (Mahler et al., 2002; Calverley et al., 2003a; Cazzola et al., 2003; Dal Negro, Pomari, Tognella, \& Micheletto, 2003; Hanania et al., 2003), and two trials have assessed the impact of the budesonide/formoterol combination (Calverley et al., 2003b; Szafranski et al., 2003). All trials showed a significant reduction in risk for moderate to severe exacerbations of 25 percent to 30 percent. This reduction in risk is similar to that observed in the trials of monotherapy with ICS mentioned previously. Several observational studies have also determined risk reductions for the combination therapy only for severe exacerbations (Burney et al., 2003; Soriano et al., 2003; Rascati et al., 2005). Almost all observational studies have found risk reductions of 35 percent to 40 percent, with one study showing a 74 percent reduction (Burney et al., 2003). 
The relevant question then asks if the combination therapy is as effective as its monotherapy components or does the combination have any additive or synergistic effects. All the clinical trials have assessed combination therapy relative to patients using each component as monotherapy or a placebo group. Three of these clinical trials have reported the reduction in exacerbations of the combination therapy relative to the ICS alone and the long-acting beta-agonist alone (Calverley et al., 2003a; Calverley et al., 2003b; Szafranski et al., 2003). Two of three trials found that the combination was equally effective as the ICS alone (Szafranski et al., 2003; Calverley et al., 2003b). In contrast, only one trial found no difference between the combination therapy and the long-acting beta-agonist alone (Calverley et al., 2003a). The other two trials found significant risk reductions in exacerbations of 23 percent to 30 percent for combination therapy compared to the longacting beta-agonist alone (Calverley et al., 2003b; Szafranski et al., 2003). Results of three meta-analytic reviews combining data from all or some of these trials also revealed the same pattern (Sin et al., 2003c; Sin et al., 2004; Nannini et al., 2003). These data suggest that it is probably the inhaled corticosteroid component that maybe integral to providing a protective effect against exacerbations.

The consistency of the beneficial effect on exacerbations of ICS component was also observed when the ICS and the long-acting beta-agonist were each compared to the placebo group. When considering the overall rate of both moderate and severe exacerbations, only one (TRISTAN) of three trials found a significant reduction for both the long-acting betaagonist and ICS component compared to placebo (Calverley et al., 2003a). When considering only exacerbations requiring oral corticosteroid courses however, only the TRISTAN trial showed a significant reduction of 29 percent for long-acting beta-agonists compared to placebo (Calverley et al., 2003a). On the other hand, all three trials showed that the ICS component significantly reduced risk in exacerbations requiring oral corticosteroid courses by approximately 30 percent compared to placebo (Calverley et al., 2003a; Calverley et al., 2003b; Szafranski et al., 2003).

\section{Pharmacoeconomic evaluations in COPD}

Drugs prevent, alleviate, or cure illness or complications, and hence can impact the economic burden of a disease. Pharmacoeconomic studies basically evaluate the economic 
impact of drug therapies, and are frequently required to demonstrate the cost-effectiveness of therapies (Drummond MF, O'Brien BJ, Stoddart GL, \& Torrance GW, 1997a). These studies need to assess not only the acquisition cost of the drug, but also the cost of the disease such as physician visits, emergency room (ER) visits, and hospitalizations (Drummond MF, O'Brien BJ, Stoddart GL, \& Torrance GW, 1997c). Depending on the perspective of the analysis, different kinds of costs can be included. For instance, only direct costs maybe important for a health insurer's perspective, but for an employer indirect costs of reduced productivity are equally relevant. Pharmacoeconomic studies can be conducted using data from randomized clinical trials, by creating decision analysis models, or by analyzing claims databases (Drummond MF, O'Brien BJ, Stoddart GL, \& Torrance GW, 1997b)

At least four studies have established the cost-effectiveness of ipratropium. The comparators of interest in these studies were mostly theophylline or albuterol. Three studies used retrospective claims data (Sclar, Legg, Skaer, Robison, \& Nemic, 1994; Hilleman et al., 2000; Jubran et al., 1993), while the fourth used data from a randomized clinical trial (Friedman et al., 1999). Two of three database studies compared only costs between patients initiated on ipratropium, and not effectiveness measures. Sclar et al. found that patients initiated on monotherapy with albuterol, theophylline, or a corticosteroid in the first six months incurred significantly higher expenditures per month by $\$ 30.00$ to $\$ 50.00$ compared to ipratropium (Sclar et al., 1994). Hilleman et al. stratified patients based on disease severity level, and found that within each disease severity level, ipratropium had the lowest cost per patient per year (Hilleman et al., 2000). A difference ranging from $\$ 200$ to almost $\$ 900$ was found between the ipratropium and other therapy groups. The third database study computed costs as well as an effectiveness measure called complication-free therapy month (CFTM) over one year between ipratropium and theophylline (Jubran et al., 1993). A CFTM was defined as a month without an unscheduled visit to a physician and without a clinical exacerbation or a complication of therapy. Similar to previous studies, ipratropium was found to cost less (annual cost $=\$ 932$ vs. \$1,373) and have greater CFTMs (11.30 vs. 10.68) than theophylline, thereby being cost-effective as well as cost-saving. Both the Hilleman et al. study and the latter study showed that the lower acquisition cost of theophylline compared to ipratropium did not lower overall costs because of increased clinic visits and hospitalizations due to increased toxicity. Friedman et al. assessed the cost-effectiveness of a 
combination of ipratropium and albuterol compared to each of the individual drugs using data on costs and efficacy from two clinical trials (Friedman et al., 1999; Petty, 1995). The treatment arms containing ipratropium were dominant in the study with $33 \%$ fewer exacerbations and consequent decreases in resource use (hospital days, antibiotic use and corticosteroid use) and costs compared to albuterol. The mean total per patient cost was \$269, \$156, and \$197 for albuterol, ipratropium and ipratropium-albuterol combinations, respectively. The combination of ipratropium and albuterol versus ipratropium alone was not significantly different indicating similar cost-effectiveness.

Following these preliminary studies, ipratropium or the combination of ipratropium and albuterol has been established as first-line therapy in COPD. Therefore, future pharmacoeconomic studies should focus on comparing new therapies such as long-acting beta-agonists to ipratropium or to the combination of ipratropium and albuterol. Three pharmacoeconomic studies of long-acting beta-agonists have been identified, two conducted alongside clinical trials (Jones, Wilson, \& Sondhi, 2003b; Hogan, Geddes, \& Gonzalez, 2003) and one using a claims database (Howard KB, McLaughlin, Pathak DS, Okamoto L, \& Bowers B, 2001). One study assessed the cost-effectiveness of salmeterol relative to placebo and not ipratropium (Jones et al., 2003b). Results showed salmeterol to have higher total costs of $£ 90.10$ (95\% CI: $£ 20.09-£ 154.11)$ as well as increased effectiveness on all outcome measures over 16 weeks. A pharmacoeconomic study of formoterol however, assessed costs and effectiveness relative to ipratropium over 12 weeks (Hogan et al., 2003). A major limitation of the study precluding the use of the results in decision-making is that no a priori economic data were collected except for the use of rescue medications, and only cost of study drugs and rescue medications were considered in this analysis. Incremental analyses showed that formoterol $12 \mu \mathrm{g}$ cost $\$ 1,611$ per additional change in $\mathrm{FEV}_{1}$ and $\$ 25$ per additional change in St. George's Respiratory Questionnaire (SGRQ) units compared to ipratropium. However, in this trial, the difference between ipratropium and formoterol on both measures of $\mathrm{FEV}_{1}$ and SGRQ units were statistically but not clinically significant (Dahl et al., 2001). In addition, data from another formoterol trial shows equal efficacy with ipratropium on similar outcomes (Wadbo et al., 2002).

Friedman et al. conducted a hypothetical analysis based on data from two different trials of ipratropium and salmeterol (Friedman \& Hilleman, 2001). The trials found similar 
reductions in exacerbations for salmeterol and ipratropium (37\% vs. 33\%, respectively). The authors estimated health care costs per patient per year based on a prior pharmacoeconomic analysis, and found that salmeterol would have higher costs $(\$ 1,059)$ compared to ipratropium (\$788). The costs for salmeterol were estimated and not empirically assessed. One study compared six-month COPD-related costs between patients initiated on salmeterol or ipratropium using the Pharmetrics database, which includes medical and prescription claims from several managed care plans (Howard KB et al., 2001). The choice of drug therapy did not impact COPD-related costs or total health care costs. The study also found that ipratropium users had a significantly higher risk for a COPD-related ER visit $(\mathrm{OR}=1.9$, 95\% CI: 1.1-3.2) or hospitalization (OR $=1.5,95 \%$ CI: $1.2-2.0)$. Therefore, the authors concluded that the higher acquisition cost of salmeterol was justified, since it prevented the need for higher resource items like ER visits and hospitalizations, thereby showing no differences in either COPD-related or total costs when compared to ipratropium. However, the authors do mention the possibility that the ipratropium group was probably sicker due to higher pre-index costs. Neither the study design nor the analyses attempted to account for this possible source of selection bias. In addition, exacerbations not requiring hospitalizations were not assessed, which has been shown in clinical trials to not differ appreciably between these two therapy groups.

Two studies have assessed the costs and effects of ICS in COPD. One study conducted a pharmacoeconomic analysis (Ayres, Price, \& Efthimiou, 2003) alongside a six-month clinical trial (Paggiaro et al., 1998) compared to usual care. Study results showed fluticasone to be cost-saving when evaluated from a societal perspective (direct and indirect costs were included), while it was cost-effective from a payer's perspective (only direct costs were included). The second study used a Markov model to answer the question of which population of patients with COPD, based on disease severity levels, would most benefit from treatment with ICS (Sin, Golmohammadi, \& Jacobs, 2004). A Markov model was constructed using a time horizon of three years and health states corresponding to disease severity stages of COPD according to the American Thoracic Society (ATS) criteria(American Thoracic Society, 1995). Four strategies were compared for patients with COPD: treat no patients with ICS, treat all patients with ICS, treat only stage 2 or 3 (moderate-to-severe) patients with ICS, or treat only stage 3 (severe) patients with ICS. A 
0.01 difference in quality-adjusted life year (QALY) was found between treating no patients with COPD with ICS or treating only the moderate and severe populations. The incremental cost-effectiveness ratio was $\$ 11,000$ per QALY gained for treating only severe patients, and $\$ 17,000$ per QALY gained for treating both moderate and severe patients compared to treating no patients with COPD. Limitations of this analysis relate to assumptions of the model such as rate of lung function decline due to ICS, and not accounting for adverse effects.

\section{Rationale for Use of West Virginia and Kentucky Medicaid Claims Data}

Administrative claims databases have become important sources of data for pharmacoepidemiologic research, which generally refer to effectiveness studies of drugs. Randomized controlled trials are invaluable for determining the efficacy of drug therapies that determine whether the drug works at all compared to no therapy or standard therapy. These trials are usually conducted in a highly selected patient population, under a uniform protocol, and increased monitoring. In contrast, effectiveness aims to assess the impact of the drug in all subsets of the population for whom the drug in indicated, in different settings where practice patterns vary from recommended guidelines, and on outcomes seldom assessed in clinical trials such as overall medical costs.

The Medicaid program provides coverage for hospitalizations, ER visits, outpatient visits, and prescription drugs. Thus, researchers can capture a person's health care encounters over a longitudinal time frame dependent on data availability, and determine inter-relationships between different components of health care use. The Medicaid claims data will be used for the proposed study, and is considered appropriate for several reasons. The outcomes of interest in the study such as hospitalizations, exacerbations not requiring hospitalization, and costs can be obtained from the database at a relatively low cost relative to that incurred in a clinical trial. Also, the Medicaid program provides comprehensive prescription drug benefits to a low-income population that rarely have other sources of prescription drug insurance, thus ensuring minimal out-of-system prescription drug use. In COPD, additional use of prescription drugs reflects greater severity, and hence the advantage of complete capture of prescription drug use in the Medicaid database is important to the study design. 
The Medicaid program is a health insurance program for the indigent, disabled, and members of families with dependent children, jointly funded by the federal and state government. Thus, the population is relatively homogeneous, and represents a sub-group of lower socioeconomic status. This is particularly relevant for the COPD population, since low socioeconomic status is considered a risk factor for developing COPD, probably reflecting exposures to indoor and outdoor air pollutants, crowding, poor nutrition, or other factors related to socioeconomic status (Pauwels et al., 2001).

The Medicaid population of West Virginia and Kentucky will specifically be used for the proposed study. These states are of particular importance given that COPD is the third or fourth leading cause of death in both states (West Virginia, 2005; Kentucky, 2005). Also, the western, mountainous states in the U.S. have the highest COPD death rates, while West Virginia and Kentucky are the only two states in the South Atlantic Region that have rates as high as these western states (NHLBI Fact Sheet, 2005). The presence of dual eligibility of Medicaid patients in the Medicare system in those over 64 years of age does not allow capture of all medical and prescription claims for this subset. Accordingly, the present study will only be able to analyze data of those between 35 to 64 years of age. This can be considered particularly relevant due to two reasons. The study population that includes the states of West Virginia and Kentucky has one of the highest rates of smoking among youths, which leads to a higher prevalence of COPD in a relatively younger population. Also, demonstrating a beneficial impact of drug therapy on mortality will be particularly useful for a younger population. 


\section{NEED FOR THE STUDY}

Goals of management in COPD include lessening airflow limitation, preventing and treating secondary medical complications, such as hypoxemia and infections, and decreasing respiratory symptoms and improving HRQol. A fourth reasonable goal in a cost-conscious environment is to use health-care resources efficiently given the enormous economic impact of the condition (Pauwels et al., 2001). To facilitate this goal, economic information regarding treatments should be made available for incorporation into treatment guidelines. For example, information from earlier pharmacoeconomic analyses (Hilleman et al., 2000; Jubran et al., 1993; Sclar et al., 1994) helped establish ipratropium as the drug of choice in COPD that complemented earlier treatment guidelines (Friedman, 1995).

Recent developments such as the introduction of new bronchodilators and new evidence of the importance of ICS have expanded pharmacotherapy options for managing COPD. Guidelines are being updated and new evidence is being incorporated every year. For example, the 2001 guidelines recommend regular treatment with long-acting bronchodilators for moderate-to-severe COPD only on the basis that it was more convenient. The 2003 update now recommends regular treatment with long-acting bronchodilators for moderate-tosevere COPD given evidence that it was more effective and convenient than short-acting bronchodilators. Pharmacoeconomic evidence supplements the development of guidelines, and is required in a cost-conscious environment.

A lack of economic evaluation studies have been identified in the literature, primarily because of a prior lack of pharmacotherapeutic options (Ramsey, 2000). Since 2002, there have been a few pharmacoeconomic evaluations in COPD to address this gap in the literature. Prior studies of the cost-effectiveness of COPD therapies, such as long-acting beta-agonists, have not compared all relevant comparators simultaneously thus providing an inadequate picture of their cost-effectiveness. Also, studies that did accommodate several comparators have not assessed costs and lack assessment on other important outcomes. Pharmacotherapies in COPD act through mechanisms other than improvement in $\mathrm{FEV}_{1}$ to produce improvements in terms of symptom scores, exercise tolerance, quality of life, and dyspnea. Therefore, several experts acknowledge that it is important to assess the effectiveness of these therapies on outcomes other than physiologic measures (Gross, 2003). Health care resources such as unscheduled doctor visits or ER visits, acute exacerbations, and 
mortality have been identified as important outcomes. Available literature using claims databases have generally tended to focus on outcomes such as hospitalization and mortality. Total resource use or exacerbations not requiring hospitalizations are stronger outcomes, since they are relevant to the payer and patient, and also its use in assessing effectiveness of therapies may have more power than those of the other outcomes (Burney et al., 2003).

A major disadvantage of conducting pharmacoeconomic studies using claims databases is the lack of randomization between therapy groups. This leads to a selection bias with the result that a more severe group may receive a particular type of therapy different from a less severe group. The studies in COPD using claims databases have not used appropriate techniques to account for a selection bias between patients receiving particular therapies. Therefore, there is a need to conduct separate evaluations of pharmacotherapies in COPD based on disease severity levels, using appropriate econometric techniques to account for selection bias.

Prior to conducting an economic evaluation, a cost-of-illness study is important to identify cost drivers in the Medicaid system. No study has yet been conducted in this population, and is required given their low socioeconomic status. Furthermore, the high prevalence and mortality rates in West Virginia and Kentucky justify using Medicaid data from these states. Cost estimation of the COPD population by their asthma status will help to identify high-risk groups if any, for targeted interventions.

This study will be conducted in three phases. Phase I will assess the costs incurred by West Virginia and Kentucky Medicaid due to COPD, and also determine if a concomitant diagnosis of asthma increases costs for recipients with COPD. Phase II will determine clinically relevant and economic outcomes of maintenance pharmacotherapy in COPD, and Phase III will determine the relationship between ICS use in COPD and mortality. 


\section{STUDY GOALS AND OBJECTIVES}

Phase I

The goal of Phase I is to identify costs incurred by recipients with COPD in the West Virginia and Kentucky Medicaid populations.

Research Objective 1: To estimate mean incremental costs incurred due to COPD by recipients enrolled in West Virginia and Kentucky Medicaid.

Research Objective 2: To compare mean COPD-related costs among recipients with COPD with and without concomitant asthma enrolled in West Virginia and Kentucky Medicaid.

FIGURE 2: Conceptual Framework for Phase I

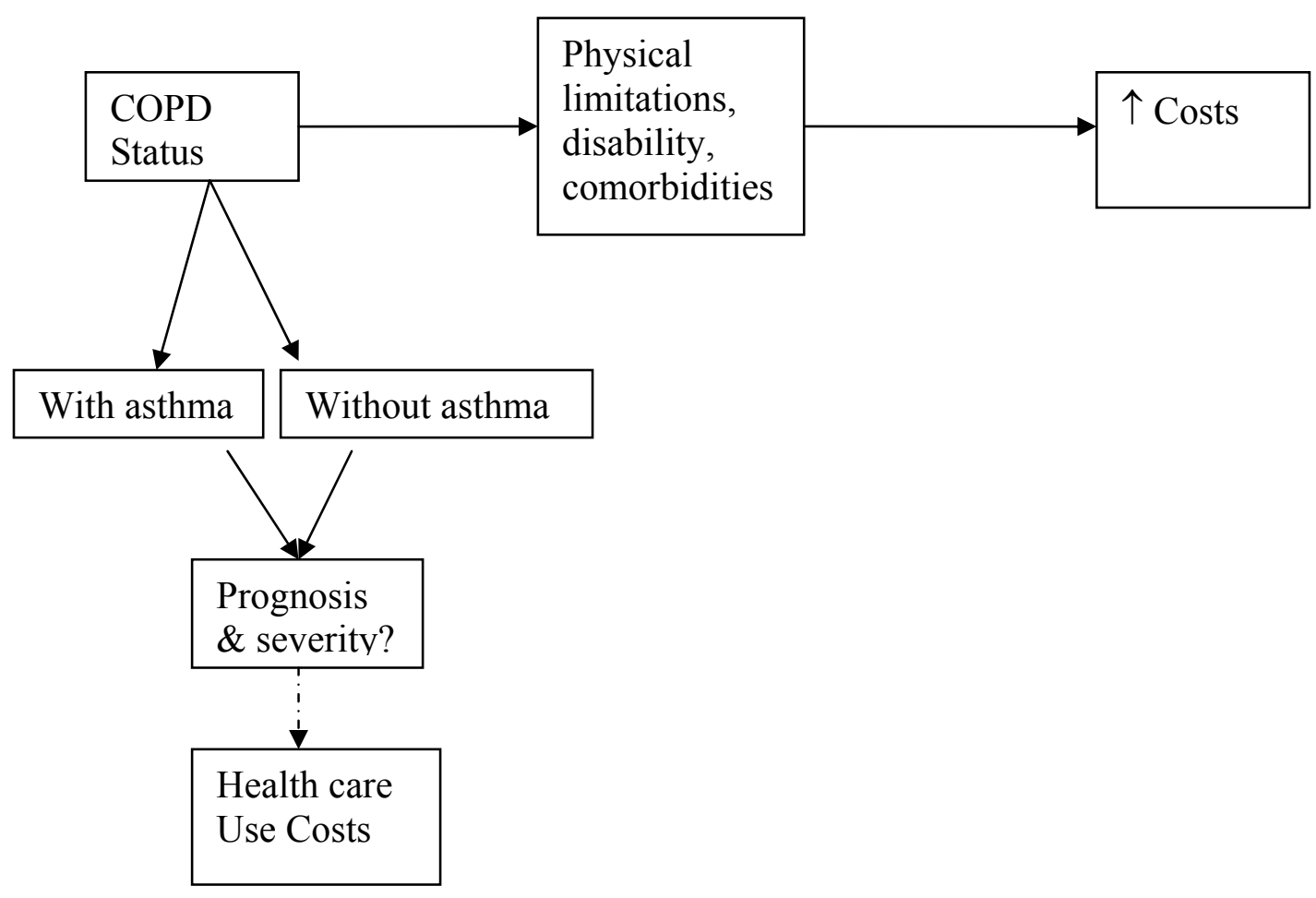




\section{Phase II}

The goal of Phase II is to compare clinically relevant and economic outcomes among alternative pharmacotherapy approaches for maintenance management of COPD.

\section{Part 1}

Research Objective 3: To compare the risk and frequency of exacerbations between recipients with COPD who initiated maintenance therapy with ipratropium or monotherapy with a long-acting beta-agonist.

Research Objective 4: To compare COPD-related costs between recipients with COPD who initiated maintenance therapy with ipratropium or monotherapy with a long-acting betaagonist.

\section{Part 2}

Research Objective 5: To compare the risk of a severe exacerbation among recipients with COPD who initiate therapy with a combination of an inhaled corticosteroid and a long-acting beta-agonist, inhaled corticosteroid alone, inhaled long-acting beta-agonist alone, or only short-acting bronchodilators.

Research Objective 6: To compare the frequency of moderate exacerbations among recipients with COPD who initiate therapy with a combination of an inhaled corticosteroid and a long-acting beta-agonist, inhaled corticosteroid alone, inhaled long-acting beta-agonist alone, or only short-acting bronchodilators.

Research Objective 7: To compare COPD-related costs among recipients with COPD who initiate therapy with a combination of an inhaled corticosteroid and a long-acting betaagonist, inhaled corticosteroid alone, inhaled long-acting beta-agonist alone, or only shortacting bronchodilators. 
FIgURE 3: Conceptual Framework for Phase II

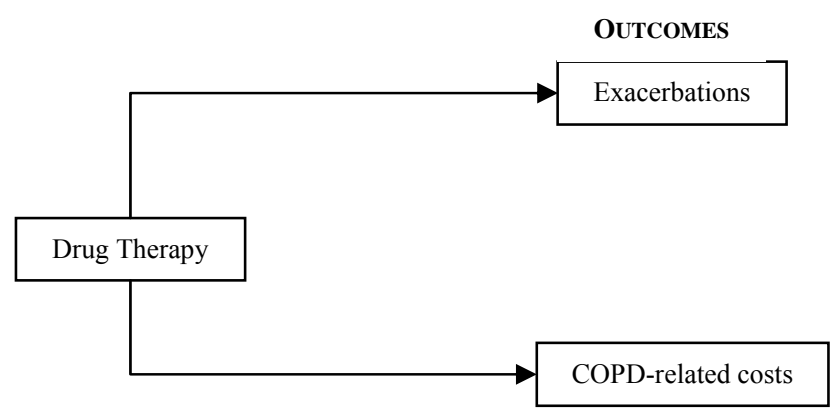

\section{Phase III}

The goal of phase III is to determine the association between use of inhaled corticosteroid $12 \mathrm{~d}$ all-cause mortality using two different study designs, and to ascertain if any association found is mediated by exacerbations.

Research Objective 8: To compare the odds of exposure to inhaled corticosteroids between those who died and those who did not die during the study period (case-control study design). Research Objective 9: To conduct a mediation analysis to examine the relationship between use of inhaled corticosteroid, exacerbations and death (cohort study design).

\section{FIGURE 4: Conceptual Framework for Phase III}

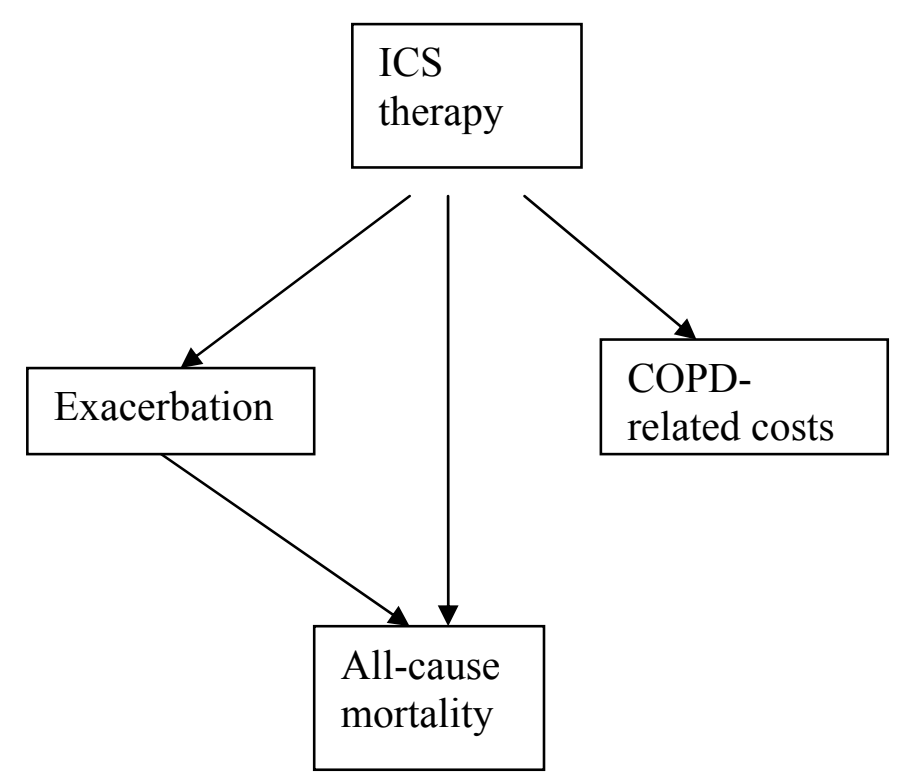




\section{STUDY SIGNIFICANCE}

The proposed study will help to identify cost drivers and high-risk groups in the Medicaid sub-population. These data can be used to develop targeted interventions, especially smoking cessation programs for these sub-groups. In addition, the proportion of the COPD population with concomitant asthma will be identified, which can provide an indication of the appropriateness of therapy with ICS. Also, assessment of the effectiveness and costs of therapies are particularly important in COPD currently due to the availability of new drugs. The combination of ipratropium and albuterol or ipratropium alone is a relatively inexpensive option for maintenance therapy in COPD. The high acquisition cost of long-acting betaagonists needs to be justified in terms of the outcomes when compared to the inexpensive option of ipratropium. Evidence from observational studies regarding the impact of ICS on mortality is not supported by clinical trials. This study will assess the impact of these drugs on mortality, as well as attempt to provide an explanation for this effect by determining if this effect is mediated through the effect of these drugs on exacerbations. This will provide preliminary evidence for any beneficial effect that may be observed in future clinical trials of ICS. 


\section{CHAPTER 2: LiterATURE REVIEW}

Chapter 2 provides an overview of the literature pertinent to the understanding of the goals and objectives of the study. Specifically, this chapter provides an overview of the disease state, outlines pharmacotherapeutic strategies for maintenance treatment, and provides empirical evidence of the efficacy and effectiveness of pharmacotherapies.

\section{INTRODUCTION TO THE DISEASE STATE- COPD}

\section{Pathogenesis, Pathophysiology, and Symptoms}

One of the important aspects of the new COPD definition is acknowledgement of the fact that inflammation is the driving mechanism behind the abnormalities noted in the airways, parenchyma, and pulmonary vasculature of patients with COPD (Pauwels et al., 2001). Inflammation results from inhalation of toxic substances, primarily chemicals in cigarette, cigar, and pipe smoke. These substances activate inflammatory cells such as macrophages, T-lymphocytes (CD8+), and neutrophils. The activated inflammatory cells in turn cause elevated levels of proteases and certain cytokines such as leukotriene $\mathrm{B}_{4}$, interleukin (IL)-8 and tumor necrosis factor (TNF) $-\alpha$, which are ultimately responsible for structural changes in the airways (Briggs, Jr., 2004). These inflammatory mediators perpetuate the inflammatory processes even after cessation of exposure to noxious particles. In addition to inflammation, an imbalance of proteinases and antiproteinases in the lungs, and oxidative stress are also considered to be important in the pathogenesis of COPD.

The inflammatory processes described above lead to pathologic changes in COPD that occur in four compartments of the lungs to varying degrees in patients with COPD: central airways (trachea, bronchi, and bronchioles greater than 2 to $4 \mathrm{~mm}$ in internal diameter), peripheral airways (small bronchi and bronchioles less than $2 \mathrm{~mm}$ in internal diameter), lung parenchyma, and pulmonary vasculature (Pauwels et al., 2001). Changes in the central airways include enlargement of mucus-secreting glands (mucus gland hypertrophy), and an increase in the number of goblet cells associated with mucus hypersecretion (goblet cell hyperplasia). The peripheral airways experience repeated cycles of injury and repair of the airway wall that increases the collagen content and forms scar tissue. These changes modify 
the structure of the airway wall that narrows the lumen and produces fixed airway obstruction. Destruction of the lung parenchyma involves dilatation and destruction of the respiratory bronchioles generally characterizing emphysema. The result of this process is that a loss of lung elastic recoil and intraluminal pressure occurs in the terminal airways, and causes small airways to lose their patency leading to airflow limitation (Mannino, 2003).

This emphysematous destruction can occur diffusely in some parts of the lung or throughout the entire lung depending on the stage of the disease. Thickening of the vessel wall occurs because of increases in smooth muscle cells, proteoglycans and collagen, and infiltration by inflammatory cells leading to pulmonary vascular changes.

Pathologic changes described above result in physiologic changes such as increases in smooth muscle tone, mucus hypersecretion, cilia dysfunction, and airflow limitation (Briggs, Jr., 2004). Patients with COPD can no longer exhale efficiently and, in response, lung volume increases (pulmonary hyperinflation), especially during exercise, trapping air, decreasing inspiratory capacity, and inducing dyspnea (breathlessness). For a patient with advanced COPD, the complex of peripheral airways obstruction, parenchymal destruction, and pulmonary vascular abnormalities reduces the lung's capacity for gas exchange, producing hypoxemia (reduced oxygen) and hypercapnia (increased carbon dioxide). This defect in gas exchange is the primary reason for chronic respiratory failure. Pulmonary hypertension is the major cardiovascular complication of COPD, and is associated with the development of cor pulmonale. The physiologic changes noted in COPD give rise to characteristic symptoms such as cough, sputum production, and dyspnea on exertion (Pauwels \& Rabe, 2004). Other relatively non-specific symptoms include wheezing and chest tightness that may vary between days and over the course of a single day.

\section{Risk Factors}

Inflammation in COPD results from three categories of environmental risk factors: tobacco smoke, occupational exposure, and indoor and outdoor air pollution (Pauwels et al., 2001). Tobacco smoke is probably the only risk factor that has substantial evidence for a causal rather than a statistical association with COPD risk. Cigarette smokers by far have greater decreases in the annual rate of lung function decline, more respiratory symptoms, and higher death rates than non-smokers (Anthonisen et al., 2002). Pipe and cigar smoking does 
not impact morbidity and mortality rates as high as cigarette smoking but it is still higher than that due to not smoking (Pauwels et al., 2001). Intense exposure to occupational agents such as dust, gases, irritants or fumes for sufficiently extended periods of time are associated with an increased risk of developing COPD, independently of cigarette smoking (Kauffmann, Drouet, Lellouch, \& Brille, 1979). The effect is additive in the presence of cigarette smoking. Pollution from outdoor sources is considered to be very small and has less evidence compared to that from indoor sources. Indoor air pollution from biomass fuel, burned for cooking and heating in poorly ventilated dwelling results in high levels of particulate matter that activate the inflammatory processes in COPD (Behera \& Jindal, 1991) (Samet, Marbury, \& Spengler, 1987).

The high variation in susceptibility of COPD between individuals has led to the belief that there are genetic or host factors that are responsible for the development of COPD. Individuals with a severe deficiency of $\alpha_{1}$-antitrypsin, a major inhibitor of serine proteases, have increased risk of developing COPD (Konzem SL \& Stratton MA, 2002). Smoking in these individuals increases the risk appreciably, and also results in earlier age of onset compared to similar individuals who do not smoke. Other host factors such as airway hyperresponsiveness and asthma are also identified as important risk factors (Pauwels et al., 2001). Exposure to cigarette smoke and occupational agents are also known to cause an increase in airway hyper-responsiveness, further demonstrating the interaction between host and environmental factors. Abnormal lung growth during gestation or due to childhood exposures is also considered a host factor (Stein et al., 1997) (Todisco et al., 1993) (Morgan, 1998).

Other environmental influences such as a history of severe childhood respiratory infections leads to reduced lung function and increased respiratory symptoms in adulthood (Pauwels et al., 2001). Also, low socioeconomic status is associated with an increased risk of developing COPD (Prescott, Godtfredsen, Vestbo, \& Osler, 2003). This variable may probably reflect increased exposure to indoor and outdoor air pollution, crowding, or poor nutrition (Prescott \& Vestbo, 1999). 


\section{Diagnosis and Severity Staging}

A potential diagnosis of COPD should be considered in any patient who has cough, sputum production, dyspnea, or a history of exposure to risk factors for the disease (cigarette smoking, and/or exposure to environmental or occupational pollutants) (Pauwels et al., 2001). Physiological confirmation of a diagnosis of COPD should be done using spirometry. Spirometry measures the maximum volume of air forcibly exhaled from the point of maximal inhalation (FVC) and the volume of air exhaled during the first second of this maneuver $\left(\mathrm{FEV}_{1}\right)$. The ratio of these two measurements is then calculated. A diagnosis of COPD is confirmed in those with a post-bronchodilator $\mathrm{FEV}_{1}<80 \%$ of the predicted value in combination with an $\mathrm{FEV}_{1} / \mathrm{FVC}<70 \%$. The values of FEV1 and $\mathrm{FVC}$ are usually represented as a percentage of predicted values of $\mathrm{FEV}_{1}$ because universally applicable reference values for $\mathrm{FEV}_{1}$ and $\mathrm{FVC}$ are not available.

Once diagnosed, there are no widely accepted staging or severity scoring systems for patients with COPD, and results of the spirometry testing are generally used for staging and monitoring the disease course. The disease staging based on level of $\mathrm{FEV}_{1}$ and $\mathrm{FVC}$ is intended to serve as a general approach to management due to the fact that management of COPD is largely symptom-driven with only an imperfect relationship between the degree of airflow limitation and symptoms (Jones et al., 2003a). Since spirometric tests measure only a small aspect of the effects of COPD, additional testing such as measurement of lung hyperinflation, and documentation of comorbidities such as chronic respiratory failure are used to further determine the disease severity level of a COPD patient (Pauwels et al., 2004). The recent GOLD severity staging system is based on post-bronchodilatory $\mathrm{FEV}_{1}$ values (Table 2) (Fabbri \& Hurd, 2003). It should be noted that only 18.5 percent of patients classified as stage 0 actually progress to more severe airflow limitation at 15 years (Vestbo \& Lange, 2002). 
TABLE 2: Classification of severity of COPD

\begin{tabular}{|c|c|c|c|c|}
\hline \multirow[t]{2}{*}{ Stage } & \multicolumn{2}{|c|}{ Spirometric Abnormality } & \multirow[t]{2}{*}{ Characteristic symptoms } & \multirow[t]{2}{*}{ Complications } \\
\hline & $\%$ predicted $F E V_{1}$ & $F E V_{1} / F V C$ & & \\
\hline 0: At Risk & Normal & Normal & Cough, sputum production & \\
\hline $\begin{array}{l}\text { 1: Mild } \\
\text { COPD }\end{array}$ & $\geq 80 \%$ & $<70 \%$ & $\begin{array}{l}\text { With or without cough, } \\
\text { sputum production }\end{array}$ & \\
\hline $\begin{array}{l}\text { 2: Moderate } \\
\text { COPD }\end{array}$ & $50 \% \leq \mathrm{FEV}_{1}<80 \%$ & $<70 \%$ & $\begin{array}{l}\text { With or without cough, } \\
\text { sputum production, } \\
\text { dyspnea }\end{array}$ & \\
\hline $\begin{array}{l}\text { 3: Severe } \\
\text { COPD }\end{array}$ & $30 \% \leq \mathrm{FEV}_{1}<50 \%$ & $<70 \%$ & $\begin{array}{l}\text { With or without cough, } \\
\text { sputum production, } \\
\text { dyspnea }\end{array}$ & \\
\hline $\begin{array}{l}4: \text { Very } \\
\text { Severe } \\
\text { COPD }\end{array}$ & $<30 \%$ & $<70 \%$ & $\begin{array}{l}\text { Cough, sputum } \\
\text { production, dyspnea }\end{array}$ & $\begin{array}{l}\text { Chronic } \\
\text { Respiratory } \\
\text { Failure }\end{array}$ \\
\hline
\end{tabular}

Adapted from Updated GOLD Guidelines 2003 (Fabbri et al., 2003).

\section{Epidemiology}

The available data on the prevalence, morbidity and mortality of COPD are considered to under-estimate the total burden of disease primarily because the disease is usually diagnosed after moderate advancement has occurred and clinical symptoms become apparent (Pauwels et al., 2001). This section will specifically focus on estimates of prevalence, mortality and economic burden in the U.S.

\section{$\underline{\text { Prevalence }}$}

Prevalence estimates for COPD in the U.S. are generally obtained annually from the National Health Interview Survey (NHIS) and in 1988 and 1994 from the National Health and Nutrition Examination Surveys (NHANES) (Mannino, Homa, Akinbami, Ford, \& Redd, 2002). The two differ in that estimates from the NHIS are based on a self-reported physician diagnosis of COPD, while the NHANES is based on spirometric assessment. While the estimates were certainly higher in the NHANES, the trends in prevalence rates by age and race were similar in the two surveys. The prevalence rate for COPD overall (including both chronic bronchitis and emphysema) increased with increasing age in both NHIS (2000) and NHANES (1994) estimates. However, it should be noted that regardless of method of prevalence assessment, 57 percent (NHANES) to 70 percent (NHIS) of individuals are 
between 25 to 64 years of age. Racial differences in prevalence rates are apparent with whites having a higher prevalence rate than blacks. The NHIS showed higher prevalence rates for females compared to males while the opposite was reflected in the NHANES.

Prevalence rates as ascertained from the NHIS for the components of COPD (chronic bronchitis and emphysema) are presented for 2002 by demographic characteristics in table 3 . Different conclusions from the overall COPD prevalence can be noted for different demographic categories. Most importantly, the prevalence of emphysema is appreciably higher in those over 65 years of age and in males compared to those under 65 years and females, respectively.

TABLE 3: Prevalence ${ }^{a}$ of chronic bronchitis and emphysema by demographic characteristics in 2002

\begin{tabular}{lccc}
\hline \multicolumn{2}{l}{ Demographic Category } & Chronic Bronchitis & Emphysema \\
\hline Overall & & 44.3 & 15.2 \\
Age (years) & & & \\
& $18-44$ & 34.8 & 2.7 \\
& $45-64$ & 55.1 & 19.7 \\
\multirow{4}{*}{ Gender } & $\geq 65$ & 54.1 & 47.6 \\
& & & \\
\multirow{2}{*}{ Race } & Male & 29.3 & 18.5 \\
& Female & 58.1 & 12.2 \\
& & & \\
& White & 45.7 & 17.1 \\
& Black & 46.1 & 8.3 \\
\hline
\end{tabular}

${ }^{\mathrm{a}}$ Rate per 1000 persons

Source: National Center for Health Statistics, National Health Interview Survey 2002 (American Lung Association, 2004).

\section{Mortality}

Similar to prevalence data, mortality data also under-estimate COPD as a cause of death as the disease is more likely to be cited as a contributory rather than an underlying cause of death, or may not be cited at all. Evidence is available that shows that most decedents with COPD have their deaths attributed to other causes, primarily cardiovascular. In 1998, only 
45 percent of decedents with COPD mentioned on their death certificates had COPD listed as an underlying cause of death (Mannino et al., 1997). According to current estimates from the National Heart, Lung and Blood Institute (NHLBI), COPD and allied conditions (includes asthma and bronchiectasis) were the fourth leading cause of death in 2002 in the United States (NHLBI, 2004). On a global level COPD was ranked as the sixth leading cause of death in 1990 and is projected to rise to third by 2020 (Murray et al., 1997).

Based on estimates from the mortality component of the National Vital Statistics System, the death rate of COPD in the U.S. using ICD codes specifically for COPD was 66.9 per 100,000 corresponding to 119,054 deaths in 2000 (Mannino et al., 2002). The impact of age on death rate is exemplified in Figure 1 below. The death rates in whites are almost double those of blacks (70.1 vs. 42.9 per 100,000, respectively). Males have a higher death rate compared to females ( 82.6 vs. 56.7 per 100,000). One should note however, that the ageadjusted death rate for females has more than doubled from 1980 to 2000 from 20.1 to 56.7 per 100,000, whereas the age-adjusted death rate for males showed a modest increase from 73.0 to 82.6 per 100,000 during the same period. This trend can probably be attributed to the increase in smoking among women since the 1940s (NHLBI Fact Sheet, 2005).

\section{FIGURE 5: COPD death rates in the United States}

Source: Mortality Component of the National Vital Statistics System (Mannino et al., 2002)

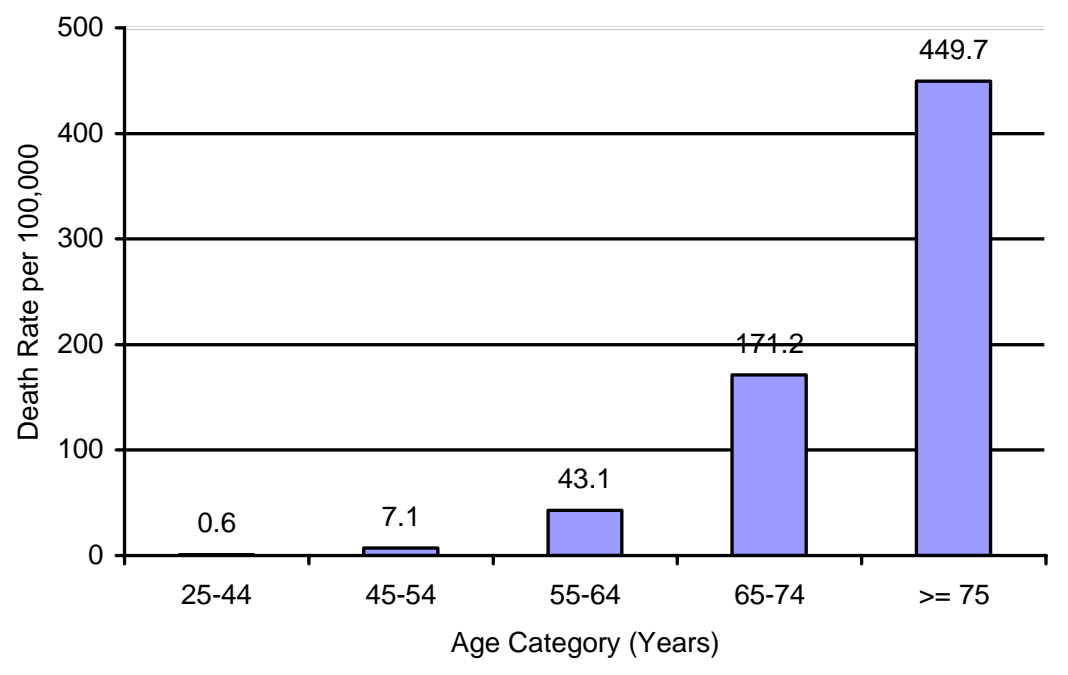




\section{Morbidity}

The morbidity of COPD is reflected in the amount of direct and indirect costs used for the management of COPD. Direct costs generally include physician visits, emergency room (ER) visits, and hospitalizations. Indirect costs include lost productivity in the form of lost workdays, and reduced productivity while at work. In 2000, COPD accounted for 726,000 hospitalizations as assessed from the National Hospital Discharge Survey (NHDS) with a rate of 40.6 per 10,000 population (Mannino et al., 2002). Similarly, the National Ambulatory Medical Care Survey (NAMCS) and the National Hospital Ambulatory Medical Care Survey (NHAMCS) estimated a total of 7.9 million physician visits (45.0 per 1000) and 1.5 million ER visits $(87.2$ per 10,000) in 2000, respectively (Mannino et al., 2002). The rates of physician visits, ER visits, and hospitalizations in the elderly above 65 years are almost twice the rates of those under 65 years. However, the COPD population under the age of 65 years account for half of all outpatient visits, nearly two-thirds of ER visits and a third of all hospitalizations for COPD (Mannino et al., 2002). Males and females do not differ in the rates of hospitalizations, ER visits or physician visits. Although whites generally account for the majority of hospitalizations, ER visits and physician visits, blacks tend to have substantially lower physician visits and higher ER visits with relatively no difference in hospitalization rates.

The limitation in airflow results in increase in lung volumes, subsequent dyspnea (breathlessness), and decreased exercise tolerance. Thus COPD patients experience marked dyspnea and general malaise that impair their ability to work, thereby rendering COPD as an extremely disabling illness. Globally, COPD is projected to rank fifth in terms of days lost to disability (Murray et al., 1997). In the U.S., COPD is responsible for 2.2 million disabilityadjusted life years (DALYs), and 0.5 million potential years of life lost. Generally considered a disease of the elderly, COPD is moderately prevalent in the working age group under 65 years (54\% to $70 \%$ ), and is responsible for considerable work loss. Analysis of the 1987 National Medical Expenditure Survey (NMES) showed that COPD patients averaged 3.6 lost workdays of which 28\% were COPD-related (Strassels et al., 2001). The impact of COPD on premature retirement was assessed in a study that determined the relationship between presence of COPD and probability of labor force participation. In that study it was assumed that absence of labor force participation was due to COPD by controlling for 
demographic (marital status, family size) and economic factors (education, area of residence) that can impact this variable. The study showed that COPD patients were $3.9 \%$ less likely to be in the labor force. Increasing severity of COPD was associated with decreased probability of being in the labor force with mild, moderate and severe COPD patients having 3.4\%, 3.9\% and $14.4 \%$ lower risk than non-COPD patients. The total absence from participation in the labor force can under-estimate the impact of COPD on work loss since patients may work but at reduced productivity levels. The impact on partial work loss or limited ability to work was assessed in the Confronting COPD U.S. survey. In this study, a third of patients reported that they were prevented from working due to COPD (Halpern et al., 2003). Eighteen percent of these patients were limited in their ability to work normally, and $6 \%$ missed time from work due to COPD. These patients reported an average of 18.7 lost workdays in the prior year. 


\section{PHARMACOTHERAPY FOR MAINTENANCE TREATMENT OF COPD}

$\mathrm{COPD}$ is defined as a partially reversible disease since $\mathrm{FEV}_{1}$ does not increase appreciably after a trial of inhaled bronchodilators or oral corticosteroids (bronchodilator reversibility testing). Even though the response to bronchodilator reversibility testing varies among patients with COPD depending on the severity and nature of the lung damage, current pharmacotherapy is recommended for all symptomatic patients. Several reasons exist for justifying the use of drugs for COPD for patients who do not have appreciable increases after bronchodilator reversibility testing (Konzem SL et al., 2002):

- Tests of bronchodilator reversibility are variable and testing on one occasion does not preclude eliciting a response on another occasion;

- As described in earlier sections, COPD is a multi-system disorder. Pharmacologic agents act through other mechanisms besides bronchodilation, and subjective relief of symptoms alone provides value to the patient; and

- Patients not responding to beta-agonists (type of drug often used for bronchodilator reversibility testing) may respond to other classes of bronchodilators such as anticholinergics or methylxanthines.

Following the rationale for using drugs for COPD, the goal of pharmacotherapy is to prevent and control symptoms, reduce the frequency and severity of exacerbations, improve health status and exercise tolerance. Two broad categories of drugs are used for maintenance treatment of COPD: inhaled bronchodilators and ICS. Table 4 provides the names of the drugs by drug class and duration of action.

\section{Medications for Maintenance Therapy of COPD}

TABLE 4: Medications for maintenance therapy of COPD (Pauwels et al., 2001) (George, 2004)

\section{BRONCHODILATORS}

\section{BETA-AGONISTS}

Short-acting: Albuterol (Salbutamol), Fenoterol, Terbutaline, Metaproterenol, Pirbuterol, Levalbuterol, Bitolterol, Long-acting: Formoterol, Salmeterol 
2. ANTI-CHOLINERGICS

Short-acting: Ipratropium, Oxitropium

Long-acting: Tiotropium

3. METHYLXANTHINES

Theophylline, Aminophylline

INHALED CORTICOSTEROIDS

Budesonide, Beclomethasone, Fluticasone, Triamcinolone

1. Bronchodilators: There are three classes of bronchodilators that are commonly used for COPD: beta-agonists, anti-cholinergics, and methylxanthines. There are substantial differences in the mode and site of action within the cell among these drug classes (Lipson, 2004). However, they are called bronchodilators because the primary effect of all these classes of drugs is relaxation of airway smooth muscle (Pauwels et al., 2001). In addition, improved lung emptying, reductions in residual volume and/or delay of the onset of dynamic hyperinflation during exercise are other changes induced by this class of drugs (Celli \& MacNee, 2004). Except for methylxanthines, the inhaled route of administration is preferred as opposed to oral administration due to lower side-effects.

Beta-agonists: Beta-agonists relax airway smooth muscle by stimulating $\beta_{2}$-adrenergic receptors, which increases cyclic adenosine monophosphate (cAMP) that produces functional antagonism to bronchoconstriction (Lipson, 2004). Depending on duration of the bronchodilation effect, this sub-group has further been categorized into short-acting and long-acting bronchodilators (See table 3 for examples). Short-acting beta-agonists have a rapid onset of action and a bronchodilatory effect for 4 to 6 hours (George, 2004). Therefore, these agents are indicated only on an as-needed basis (Pauwels et al., 2001). Long-acting beta-agonists are appropriate for maintenance therapy because of their sustained bronchodilatory action for at least 12 hours (Dougherty, Didur, \& Aboussouan, 2003). In addition, other non-bronchodilator effects of long-acting beta-agonists include reduction in neutrophils (Bloemen et al., 1997), possible decrease in bacterial colonization by increasing intracellular cAMP in the respiratory epithelium (Dowling, Johnson, Cole, \& Wilson, 1998) (Dowling et al., 1997), and increase in mucociliary transport (Johnson \& Rennard, 2001). Beta-agonists are generally safe medications, and 
have rare side-effects that include tremor, tachycardia, supraventricular arrythmias, hypokalemia, and transient clinically insignificant decreases in arterial oxygen tension (Tashkin et al., 2004). These side-effects are almost negligible with inhaled therapy, and resolve rapidly after treatment withdrawal.

Anti-cholinergics: Anti-cholinergics usually exert their bronchodilatory effect by competitively blocking the effect of acetylcholine on cholinergic receptors in bronchial smooth muscle (Lipson, 2004). Also, since these receptors mediate mucus production, antagonism by anti-cholinergics also reduces sputum production (Tashkin et al., 2004). Similar to beta-agonists, there are short and long-acting forms of anti-cholinergics, each having only one product in the U.S., ipratropium and tiotropium, respectively (George, 2004). The short-acting anti-cholinergic, ipratropium, has a slower onset of action and a prolonged bronchodilator effect compared to short-acting beta-agonists. These features have placed ipratropium as first-line maintenance therapy in COPD, and less suitable for use on an as-needed basis for immediate relief (Friedman, 1995). The short half-life of ipratropium has the disadvantage of frequent dosing. Tiotropium, on the other hand, has a substantially longer duration of action ( 24 to 36 hours), and offers several benefits over conventional therapies (Tashkin et al., 2004). The poor absorption of these agents limits the adverse effects characteristic of anti-cholinergics, such as dryness of mouth, and render these agents with an acceptable safety profile (Pauwels et al., 2001).

Methylxanthines: Methylxanthines produce bronchodilation primarily based on their ability to inhibit phosphodiesterase which increases cAMP levels (Lipson, 2004). Other mechanisms have been suggested and include inhibition of calcium ion influx into smooth muscle, prostaglandin antagonism, stimulation of endogenous catecholamines, adenosine receptor antagonism, and inhibition of release of mediators from mast cells and leukocytes (Konzem SL et al., 2002). This class of drugs is not considered as preferred treatment for COPD, and has been relegated to second tier status due to numerous toxicity problems and frequent monitoring of supratherapeutic levels. The problem is further compounded since most of the benefits of methylxanthines occur at higher doses, and toxicity is dose-related. 
2. Inhaled corticosteroids (ICS): ICS in COPD target several aspects of the inflammatory process in COPD, although this effect is much less prominent compared to asthma. The mode of action of ICS in COPD is still not fully understood but they exert their beneficial effects by binding to the intracellular glucocorticoid receptor to form a complex that enters the cell nucleus to increase or decrease gene transcription (Sin et al., 2004). The beneficial effect of ICS in COPD is only obtained at moderate to high doses that increase the possibility of side-effects (George, 2004). Side-effects include skin bruising, oropharyngeal candidiasis, cataracts, decreases in serum cortisol concentrations, and decreases in bone density thus increasing the risk for fractures and osteoporosis (Sin et al., 2003c).

\section{Inhaled Corticosteroids in COPD: Beneficial or Not?}

The controversy of whether ICS are beneficial in COPD can best be understood by describing the inflammatory markers and mediators predominant in COPD, and those that are significantly affected by ICS. Airways obstruction due to inflammation is a defining characteristic of both asthma and COPD. However, there are distinct differences in histologic and cellular characteristics of the inflammatory processes between the two diseases (Barnes, 2000b). In asthma, airway inflammation results in a predominant increase in eosinophils, and activation of mast cells and $\mathrm{CD}^{+}{ }^{+}$T-cells (Decramer \& Selroos, 2005). On the other hand, airway inflammation in COPD shows an increase predominantly in neutrophils, and activation of macrophages and $\mathrm{CD} 8^{+} \mathrm{T}$-cells (Decramer et al., 2005). In addition, cytokines are released by activation of these cells in both asthma and COPD. However, in asthma, interleukin-4 (IL-4), IL-5, and IL-13 predominate as opposed to IL-8 and tumor necrosis factor- $\alpha$ (TNF- $\alpha)$ in COPD.

ICS are extremely effective in blocking the process of eosinophilic and mast cell infiltration making them first-line therapy in asthma (Barnes, 1998). In contrast, there is evidence that ICS may actually prolong survival of neutrophils, characteristic of COPD inflammation by suppressing apoptosis of these cells (Keatings, Jatakanon, Worsdell, \& Barnes, 1997) (Culpitt et al., 1999). In addition, inflammatory cells like macrophages and $\mathrm{CD} 8^{+}$and mediators such as IL- 8 and TNF- $\alpha$ in COPD are shown to be steroid resistant. The debate of the benefit of ICS results from conflicting evidence on several aspects. Contrary to 
the studies mentioned above, some studies contend that ICS reduce neutrophil inflammation (Confalonieri et al., 1998) (Yildiz et al., 2000) and that increased levels of eosinophilic cells are found in COPD (Balzano et al., 1999) (Pesci et al., 1998). Also, relatively large concentrations of mast cells are present in the sub-epithelial layer of COPD airways (Hattotuwa, Gizycki, Ansari, Jeffery, \& Barnes, 2002), which are known to be affected by ICS. Additional benefits on other inflammatory markers are also suggested (Calverley, 2000). This evidence and the fact that no single biochemical or cellular marker can adequately describe the airway inflammation in COPD have led some investigators to hypothesize that some aspects of the inflammatory process, but not all, can be modified by ICS. However, other investigators disagree with this approach with the consequence of a major controversy regarding these drugs (Barnes, 2000a; Calverley, 2000).

To estimate the benefits of ICS therapy in COPD, it is important to consider the proportion of patients with COPD with asthma. A sub-group of patients with COPD that exhibit a significant increase in $\mathrm{FEV}_{1}$ after oral corticosteroid administration are generally described as patients with COPD with asthmatic features. This subset has been estimated to constitute 10 percent of all patients with COPD (Calverley, 2000). However, the absence of significant reversibility does not imply that these patients with COPD do not have asthma. A recent population-based study analyzed medical records of all patients with COPD enrolled in a health maintenance organization (HMO) in 1998 to estimate the proportional distribution of the sub-types of COPD (Mapel et al., 2000b). They found that almost half $(48.5 \%)$ of patients in this COPD population had asthma as either their primary or secondary diagnosis. It is important to note that almost all patients with COPD that had asthma listed as their primary diagnosis had either severe asthma with fixed airflow obstruction or were current or former cigarette smokers. Based on these findings, the authors concluded that most patients with COPD will experience some benefit from ICS (Mapel, 2004; Mapel et al., 2000b). Given the controversial role of ICS, it is important to determine the rate of use of this class of drugs compared to other classes in the general COPD population.

\section{Patterns of Pharmacotherapy Use in COPD}

Introduction of long-acting forms of inhaled bronchodilators and recent evidence of the beneficial impact of ICS in COPD have led to modifications in treatment algorithms for 
COPD. Studies of drug use patterns for COPD are presented in chronological order to enable assessment of the shift in treatment practices, due to the introduction of new treatments and evidence. Analysis of the National Medical Expenditure Survey (NMES) in 1987 by Strassels et al. showed that the most commonly prescribed drugs for COPD in the U.S. included xanthines (69.7\%), and beta-agonists (46.5\%). Ipratropium was used by only 2.6 percent of all individuals with COPD at that time (Strassels et al., 2001). The proportion of subjects using ICS was not mentioned. Hilleman et al. analyzed 1993 and 1994 data on hospital admissions, clinic visits, and pharmacy use at a university medical center in the U.S. to determine the distribution of drug use by disease severity level as defined by the American Thoracic Society (ATS) criteria (Hilleman et al., 2000). At the time of identification of the patients in the study, 100 percent of patients in moderate and severe COPD were using combination therapy (treatment with more than one drug) compared to 28 percent of patients with mild COPD. In terms of drug type, use of inhaled beta-agonists was the highest (77\% to $97 \%$ ), followed by ipratropium ( $72 \%$ to $85 \%$ ), theophylline, and steroids. Only 5 percent of patients with mild COPD used steroids (inhaled or oral), increasing to 23 percent for patients with moderate, and finally 100 percent for severe COPD. Interestingly, use of theophylline was high, 55 percent, 80 percent and 79 percent in mild, moderate, and severe COPD, respectively.

A group of researchers interviewed primary care physicians in Canada, and found that although physicians could adequately distinguish between asthma and COPD conceptually, their choice of initial drug therapy for these patients was remarkably similar (Kesten \& Chapman, 1993). Beta-agonists were chosen by 53 percent of physicians as initial therapy for COPD versus 64 percent for asthma. Ten percent of physicians chose anti-cholinergics for COPD as initial therapy compared with none for asthma, while 12 percent of physicians chose ICS as initial therapy compared with 5 percent for COPD, thus signifying a relatively small proportion adhering to recommended therapies for both disorders. These same researchers conducted a retrospective chart review in 1994 of hospitalized COPD and asthma patients to determine the medications used at admission to characterize patterns of drug used in the community (Jackevicius, Joyce, Kesten, \& Chapman, 1997). The results showed a significantly higher proportion of asthma (48\%) versus patients with COPD (35\%) being prescribed ICS at admission, whereas patients with COPD were 2.6 times more likely to use 
anti-cholinergics than asthma patients. These findings probably refute the authors' prior hypothesis that patients with asthma and COPD are treated similarly by Canadian physicians. However, the authors expected the difference for inhaled corticosteroid prescriptions to be larger based on the therapy recommendations at that time. Also, the population was categorized as having stable or unstable COPD or asthma, based on the primary reason for the inpatient admission. As such, the stable COPD sub-group was significantly less likely to receive ICS than the other three sub-groups, which shows that the unstable COPD group may appropriately have been prescribed ICS.

A study by Van Andel et al. in 1999 pooled data from ten clinical trials investigating COPD conducted in the U.S. between 1987 and 1995 with a sample size of at least 150 and comparable entry criteria. Data included the trial subjects' use of medications in the six weeks preceding trial enrollment (Van Andel, Reisner, Menjoge, \& Witek, 1999) that is considered to reflect the pattern of therapy as would be seen in clinical practice. The proportion of patients in each trial using ICS, inhaled beta-agonists, inhaled anti-cholinergics, oral theophylline, or oral corticosteroid was assessed. Except for inhaled beta-agonists, significant changes in proportion of patients using a particular drug were seen for all other types of drugs between 1987 and 1995. Most prominently, the percentage of patients with COPD using ICS increased from 13.2 percent in 1987 to 41.4 percent in 1995, while those using oral theophylline decreased from 63.4 percent to 29.0 percent during the same period. During this time interval, the use of oral corticosteroids and oral beta-agonists decreased moderately from 30.1 percent to 16.4 percent and from 11.7 percent to 4.5 percent, respectively. The use of inhaled anti-cholinergics increased from 48.2 percent in 1989 to 53.8 percent in 1995, possibly reflecting the effectiveness of ipratropium. In trend analyses adjusted for patient baseline severity and duration of disease, the direction of the trend for inhaled corticosteroid and theophylline use remained significant, while that of other drug classes were no longer significant. The authors concluded that medication use did not reflect current treatment standards, since at the time of the study (1999) there was little evidence regarding the efficacy of ICS for COPD, and theophylline was still considered an important addition to the therapy of COPD. They attributed the high rate of inhaled corticosteroid use in COPD to physicians' perceptions that some benefit was obtained from using ICS. This study did not report the use of medications by level of disease severity, which may have 
provided a better description of treatment patterns. For example, an increased use of ICS may have been seen in those with greater disease severity.

Further insights into some characteristics of patients with COPD receiving specific drug classes can be obtained from two database studies in Canada. Using a cohort of patients with COPD over 65 years, the yearly prevalence of inhaled corticosteroid use (defined as the presence of at least one prescription in a specific year) was assessed during a six year period between 1990 and 1996 using administrative medical and prescription claims data from the province of Quebec (Blais, Bourbeau, Sheehy, \& LeLorier, 2004). The prevalence increased from 42.2 percent in 1990 to 53.1 percent in $1995(\mathrm{p}=0.001$ test for trend). Determinants of initiating therapy with ICS was assessed using a case-control study design, where cases were defined as new users of ICS (defined as patients who were in the cohort for at least two years before they filled their first prescription of inhaled corticosteroid). Determinants included those with severe COPD (defined based on use of inhaled bronchodilators in the 3 months preceding the index date), those with at least one exacerbation requiring medical evaluation in the month before the index date, those who visited more than three different physicians in the three months preceding the index date, and those who consulted a respirologist in the month preceding the index date. Exacerbations were classified into five severity levels based on the level of medical intervention needed. It was found that the likelihood of initiating treatment with ICS increased as the intensity of medical intervention needed for the exacerbation increased. The authors also determined persistency on treatment with ICS and found that 54 percent were still using ICS after one year, and only 25 percent continued with treatment for at least five years. Thus, they concluded that although the prevalence of ICS use increased, the decrease in patient persistency may reflect a perceived absence of relief of symptoms by patients with COPD using these drugs. However, the objective of this study was not to assess the impact of ICS on symptoms, and the authors suggested that the costeffectiveness of ICS be evaluated.

Another study in the province of Manitoba compared drug use between patients with a diagnosis of COPD only, a diagnosis of asthma only and a concomitant diagnosis of COPD and asthma. Among patients with 6-9 physician visits, 30 percent of COPD only patients did not receive any drugs compared to 10 percent of both asthma only patients, and COPD and asthma patients (Anthonisen et al., 2005). In this study, patients with COPD only were 
significantly less likely to receive drugs compared to those with asthma or COPD and asthma. Patients with COPD and asthma had comparable rates of prescription drug use as those with only asthma. Consistent with recommendations, COPD only patients were prescribed beta-agonists and steroids less commonly than asthma only patients $(\mathrm{p}<0.001)$, while ipratropium was prescribed more often in COPD only patients $(\mathrm{p}<0.0001)$. Patients with COPD and asthma had beta-agonist and ICS prescriptions similar to patterns of asthma only patients, and patterns of ipratropium prescriptions similar to COPD only patients. Based on prescription refill data, compliance rates for COPD only patients and COPD and asthma patients were similar ( $54 \%$ vs. $56.4 \%$ ), whereas asthma only patients had a compliance rate of 31 percent.

Recent physician surveys have been conducted after publication of the GOLD guidelines in Belgium, Switzerland, and Greece to determine adherence to guidelines for COPD management. Only findings regarding pharmacologic management are mentioned in this section. The Belgian study surveyed both general practitioners (GPs) and pulmonologists, and found distinct differences in choice of therapy for a new COPD patient (Decramer, Bartsch, Pauwels, \& Yernault, 2003). Approximately 60 percent of GPs would prescribe either a long-acting beta-agonist or an inhaled corticosteroid for a new COPD patient compared to 40 percent of pulmonologists. On the other hand, a combination of a shortacting beta-agonist and a short-acting anti-cholinergic would be the first choice for 63 percent of pulmonologists compared to 35 percent of GPs. The fixed combination of a longacting beta-agonist and an inhaled corticosteroid was a first choice for 33 percent vs. 9 percent for GPs and pulmonologists, respectively. When prescriptions of ICS were analyzed by recommended indications, 49 percent of GPs vs. 25 percent of pulmonologists prescribed them to all patients with COPD while only 10 percent of GPs vs. 52 percent of pulmonologists used it in the correct indication ( $\mathrm{FEV}_{1}<50 \%$ and repeated exacerbations).

The Swiss and Greek studies surveyed only primary care physicians. Forty-nine percent of Swiss primary care physicians prescribed ICS regardless of indication although a higher proportion (25\%) prescribed these drugs systematically to patients with severe COPD (Rutschmann, Janssens, Vermeulen, \& Sarasin, 2004). Twenty-six percent and 53 percent of Swiss physicians prescribed short or long-acting beta-agonists as first-line treatment for COPD. Finally, a survey of Greek primary care physicians determined the proportions of 
each drug class prescribed for patients with COPD rather than the number of physicians who prescribed these categories of drugs (Trakada \& Spiropoulos, 2000). Results showed that among all COPD medications, the most common were inhaled beta-agonists followed by ICS, whereas anti-cholinergics comprised only 8 percent of all COPD medications. The study concluded that Greek physicians have relatively higher prescribing rates for ICS and theophylline and lower rates for anti-cholinergics, in contrast to guideline recommendations.

The Confronting COPD survey assessed the burden of COPD on an international level in patients with COPD or patients who met symptomatic criteria for COPD (Halpern et al., 2003). Self-report information from patients in the U.S. revealed that 67 percent were taking prescribed medications for COPD treatment. The most frequently used drugs were shortacting beta-agonists (44\%), whereas a dismal 26 percent reported using anti-cholinergics. Furthermore, the proportion of patients using theophyllines was higher (11\%) than those using long-acting beta-agonists (9\%). ICS were used by a third of patients, which was a lower proportion than that seen in Canada (Blais et al., 2004). The relatively lower proportions in this study may reflect a factor of the study population rather than evidence of under-treatment. The Confronting COPD U.S. study included a large majority of mild COPD or undiagnosed patients that are generally excluded from database studies. Since therapy is generally on an as-needed basis for this population, a lower than average rate of drug use may be expected in this study.

This section provided details of patterns of drug use in patients with COPD, and evidence showed a lower rate of use of anti-cholinergics and long-acting beta-agonists compared to recommended guidelines. Also, almost half of patients with COPD were prescribed ICS. This pattern was seen even in the two studies that actually measured prescription drug use in patients with COPD without a history of asthma (Van Andel et al., 1999; Blais et al., 2004). It is important to note that all of the above studies were conducted before November 2003, at which point no ICS had yet been formally approved by the Food and Drug Administration (FDA) for COPD. In November 2003, the combination product called Advair (inhaled corticosteroid, fluticasone, and a long-acting beta-agonist, salmeterol) was the first product in this class of drugs to be approved for COPD in the U.S. The well-established role of ICS in asthma has probably contributed to perceptions that they may be useful for treatment of COPD, and hence the increase in the off-label use of these drugs for COPD. The evidence of 
whether patients with COPD will benefit from treatment with ICS can only be ascertained from randomized controlled trials and observational studies, and is discussed in section III. 


\section{EFFICACY AND EFFECTIVENESS OF MAINTENANCE PHARMACOTHERAPY IN COPD}

The beneficial effect of maintenance pharmacotherapy in COPD needs to be evaluated on various outcome measures by reviewing evidence from different study designs in the literature. The evidence is presented from clinical trials, meta-analyses, and observational studies for each outcome measure. Relevant outcome measures include annual decline in $\mathrm{FEV}_{1}$, exacerbation frequency, and mortality. Other outcome measures such as lung function indices other than $\mathrm{FEV}_{1}$, exercise tolerance, dyspnea ratings, symptom score, and healthrelated quality-of-life (HRQol) will not be discussed, since the present study will not be able to assess these measures due to data availability. Some detail is provided below for outcomes of mortality and exacerbations regarding their measurement in the literature.

\section{Outcomes to assess pharmacotherapy effectiveness}

Mortality: COPD is currently the fourth leading cause of death in the U.S. (NHLBI, 2004). Thus, there is an interest in developing treatments that prevent mortality in COPD. Also, mortality in COPD is frequently under-estimated as a majority of deaths in patients with COPD are attributed to other causes such as cardiovascular, rather than due to COPD (Mannino et al., 1997). Therefore, mortality as an outcome in COPD has been measured in the literature as all-cause mortality, and not particularly restricted to COPD-related mortality. Use of all-cause mortality also eliminates the issue of validity of cause of death from death certificates, if administrative databases are used for the study (Burney et al., 2003).

Exacerbations: COPD is a progressive disease that is characterized by episodes of increased severity of symptoms from the normal day-to-day fluctuations. These episodes are generally called acute exacerbations of COPD, and are the result of a variety of causes including bacterial and viral infections, pollution events, cold weather, and interruption of regular treatment (Burge et al., 2003). Acute exacerbations of COPD lack a common definition, because of their heterogeneous etiology and pathophysiology. Additionally, they exhibit a variety of specific and non-specific symptoms (Rodriguez-Roisin, 2000). Specific symptoms include increased dyspnea and/or wheeze, productive cough with altered sputum (amount and 
purulence), sore throat, and fever. Other non-specific symptoms include malaise, fatigue, insomnia or sleepiness, and depression. Given the heterogeneous nature of exacerbations, a recent recommendation from a consensus panel has defined exacerbations "as a sustained worsening of the patient's condition from the stable state and beyond normal day-to-day variations that is acute in onset, and may warrant additional treatment in a patient with underlying COPD” (Burge et al., 2003).

Ascertainment of exacerbations can be done using daily diary cards or retrospective database sources (Burge et al., 2003). The daily diary card scores the severity of symptoms relative to a baseline state, and defines the start of an exacerbation as a period of at least two consecutive days when at least two symptoms have deteriorated. Exacerbations from retrospective databases are usually identified based on the frequency of medical visits and/or prescriptions for oral corticosteroids and antibiotics. Most clinical trials have thus operationally defined exacerbations as a worsening of respiratory symptoms that require treatment with oral corticosteroids or antibiotics, or both. A recent clinical trial adopted a different approach to define exacerbations (Oostenbrink, Rutten-van Molken, Al, Van Noord, \& Vincken, 2004; Oostenbrink \& Rutten-van Molken, 2004). Similar to other studies, a COPD exacerbation was defined as a complex of respiratory symptoms (i.e. new onset of worsening of more than one symptom such as cough, sputum, dyspnea, or wheeze) lasting for at least 3 days. The difference though, was that the severity of the exacerbation classified as mild, moderate or severe was based on ratings by the physician-investigator rather than the patient. These ratings were based on the patient's ability to function, and not on the intensity of medical services or drugs used for treating the exacerbations.

Acute exacerbations of COPD occur with a frequency of approximately one per year. As can be expected, this frequency depends on the underlying COPD severity, and some patients with moderate-to-severe COPD experience on an average at least three exacerbations per year. In the ISOLDE trial, 20 percent of patients with moderate-to-severe COPD did not experience any exacerbation during the 3 year period (Burge et al., 2000). Acute exacerbations of COPD are important, and are the primary target of intervention because of their impact on physical, psychological and economic aspects of the disease (Wedzicha \& Donaldson, 2003). Repeated exacerbations of all severity levels contribute to a more rapid decline in lung function and poorer HRQol (Seemungal et al., 1998; Donaldson, Seemungal, 
Bhowmik, \& Wedzicha, 2002). When considering the cost of exacerbations though, severe exacerbations are more important as they account for 90 percent to 95 percent of the total costs of acute exacerbations of COPD (Niederman, McCombs, Unger, Kumar, \& Popovian, 1999; Oostenbrink et al., 2004).

Table 5 elaborates on different definitions of an exacerbation used in observational studies. These studies use administrative data to characterize a moderate or severe exacerbation based on level of healthcare utilization, precluding identification of exacerbations that do not require health care use.

\section{TABLE 5: Definitions of exacerbations from observational studies of COPD}

\begin{tabular}{|c|c|}
\hline Study & Definition \\
\hline $\begin{array}{l}\text { 1. de Melo et al. (de } \\
\text { Melo et al., 2004a). }\end{array}$ & \\
\hline Moderate exacerbation & $\begin{array}{l}\text { Prescriptions for a systemic antibiotic and an oral corticosteroid on the } \\
\text { same day. }\end{array}$ \\
\hline Severe exacerbation & Hospitalization with a primary discharge diagnosis of COPD \\
\hline $\begin{array}{l}\text { 2. Fan et al. (Fan et al., } \\
\text { 2003) }\end{array}$ & \\
\hline Moderate exacerbation & $\begin{array}{l}\text { Outpatient visit with a primary ICD-9 code for COPD combined with a } \\
\text { new prescription for a } 14 \text { (or fewer) day course of either prednisone or } \\
\text { a commonly used antibiotic (amoxicillin, sulfa drugs, cephalosporins, } \\
\text { quinolones, tetracyclines, and macrolides) that was dispensed and } \\
\text { filled within } 24-48 \text { hours of the clinic visit. }\end{array}$ \\
\hline Severe exacerbation & Hospitalization with a primary discharge diagnosis of COPD \\
\hline
\end{tabular}

3. de Melo et al. (de

Melo, Ernst, \&

Suissa, 2004b).

Type 1 Dispensing of a prescription for a systemic antibiotic (aminoglycosides, cephalosporins, macrolides, penicillins, fluoroquinolones, tetracycline derivatives or cotrimoxazole)

Type 2 Dispensing of a prescription for a systemic antibiotic and an oral corticosteroid on the same day

Type 3 Hospitalization with a primary discharge diagnosis of COPD

4. Blais et al.

(Beauchesne,

Lalande, Fillion, \&

Blais, 2005). 
Level 1 No exacerbations

Level 2 Exacerbation requiring only antibiotic treatment (ciprofloxacin $500 \mathrm{mg}$ or $750 \mathrm{mg}$, amoxicillin $250 \mathrm{mg}$ or $500 \mathrm{mg}$, ampicillin $250 \mathrm{mg}$ or 500 $\mathrm{mg}$, trimethoprimsulfamethoxazole $20 \mathrm{mg}$ to $100 \mathrm{mg}$, 80 to $400 \mathrm{mg}$, or $160 \mathrm{mg}$ to $800 \mathrm{mg}$, cefaclor $250 \mathrm{mg}$ or $500 \mathrm{mg}$, cefadroxil $500 \mathrm{mg}$, cefixime $200 \mathrm{mg}$ or $400 \mathrm{mg}$, cefprozil $250 \mathrm{mg}$ or $500 \mathrm{mg}$, cefuroxime $250 \mathrm{mg}$ or $500 \mathrm{mg}$, cephalexin $250 \mathrm{mg}$ or $500 \mathrm{mg}$, azithromycin 250 $\mathrm{mg}$, clarithromycin $250 \mathrm{mg}$ or $500 \mathrm{mg}$, and erythromycin $250 \mathrm{mg}$ or more.

Level 3 Exacerbation requiring a course of oral corticosteroids with or without antibiotics

Level 4 Exacerbation requiring a visit to an emergency department

Level 5 Exacerbation requiring hospitalization

\section{Inhaled Bronchodilators: Long-acting Beta-agonists and Short-acting Anti-cholinergics}

Ipratropium, a short-acting bronchodilator (anti-cholinergic) has been considered as firstline therapy in COPD before the introduction of long-acting bronchodilators. The recent update to the GOLD guidelines suggests that long-acting beta-agonists and long-acting anticholinergics are more effective and convenient for maintenance therapy than the short-acting anti-cholinergic, ipratropium, based on data from clinical trials (Fabbri et al., 2003). The evidence for long-acting beta-agonists relative to ipratropium in particular is inconsistent, and will be reviewed in this sub-section. The long-acting anti-cholinergic, tiotropium, cannot be evaluated relative to ipratropium because the drug was launched in the U.S. in 2004 and data for the proposed study is available only till 2003. The results of the Lung Health Study established that inhaled bronchodilators do not modify the rate of decline of $\mathrm{FEV}_{1}$ or mortality, and hence only exacerbations will be considered for this class of drugs.

\section{Clinical Trials and Meta-Analyses:}

Compared to numerous trials conducted in comparison to placebo, only four comparative trials have been identified for the long-acting beta-agonists, salmeterol and formoterol, (two each). All four trials were parallel group, 12-week trials, and included lung function, dyspnea score, or exercise capacity as the primary efficacy variable (Mahler et al., 1999; Rennard et al., 2001; Dahl et al., 2001; Wadbo et al., 2002). Only three trials (2 salmeterol and 1 formoterol) have reported exacerbations as secondary outcomes that differed in the definition and manner of reporting (Mahler et al., 1999; Rennard et al., 2001; Dahl et al., 2001). The salmeterol studies generally used a definition based on need for medical 
treatment, while the formoterol study used a definition based on symptoms and need for medical treatment. In the trial of Mahler et al., salmeterol had a significantly longer time to an exacerbation relative to both ipratropium and placebo (Mahler et al., 1999). Also, the proportions of patients experiencing exacerbations were lowest for salmeterol (20.7\%) compared to ipratropium (30.8\%) and placebo (32.9\%), which was not statistically tested. Rennard et al. only considered exacerbations that required treatment with oral corticosteroids for 14 days or less, and found that the proportion of patients with exacerbations did not differ among the treatment groups (Rennard et al., 2001). Dahl et al. specified three levels of an exacerbation (mild, moderate, and severe). Only the first level (mild exacerbations) reported as percentage of bad days was significantly smaller in the formoterol treatment arm compared to both placebo and ipratropium (Dahl et al., 2001).

Two studies have pooled data from clinical trials in a meta-analysis. However, these data have been summarized for long-acting beta-agonists compared to placebo. Both metaanalyses have shown inconsistent results. Sin et al. pooled data from eight randomized, controlled trials to determine the magnitude of risk reduction for exacerbations relative to placebo. Of the eight trials, only one showed a significant reduction of 37 percent (Sin et al., $2003 \mathrm{c}$ ). The pooled risk ratio however, showed a significant reduction of 21 percent (RR: 0.79; $95 \%$ CI: 0.69 to 0.90 ). The second meta-analysis, a Cochrane review, concluded that long-acting beta-agonists did not impact the rate of exacerbations compared to placebo (Appleton, Poole, Smith, Veale, \& Bara, 2002). Unlike the meta-analysis by Sin et al., the Cochrane review restricted the pooled study population to those with $<15 \% \mathrm{FEV}_{1}$ reversibility after bronchodilator administration. Also, the review only included trials for salmeterol, since data from formoterol trials were not yet published at the time of the review. The study found an insignificant risk ratio of 0.69 (95\% CI: 0.47 to 1.03 ) for $50 \mu \mathrm{g}$ of salmeterol and 0.98 (95\% CI: 0.64 to 1.52$)$ for $100 \mu \mathrm{g}$ of salmeterol in the incidence of COPD exacerbations.

One can hypothesize that patients with COPD with high symptom scores, high use of rescue medications, high dyspnea scores, and poorer quality-of-life and lung function would incur higher costs. Although a positive correlation has been established for quality of life (Fan et al., 2002) and lung function as measured by $\mathrm{FEV}_{1}$ (Hilleman et al., 2000), the same has not been shown for the other outcomes. However, it is plausible that patients with COPD 
who incur higher costs have high symptom scores, dyspnea scores, and use of rescue medication. Thus, COPD-related costs can be considered a summative measure that represents these outcomes. The proposed study seeks to compare long-acting beta-agonists to ipratropium on COPD-related costs. Thus, data on lung function, exercise capacity, dyspnea, symptom scores, and HRQol will be reviewed from the four trials comparing longacting beta-agonist to ipratropium mentioned above. The equal efficacy of long-acting betaagonists to ipratropium is also reflected in these four outcome measures. Of the four trials, only one trial found significant differences between long-acting beta-agonists and ipratropium in lung function. This trial reported normalized $\mathrm{FEV}_{1} \mathrm{AUC}$ (area under the curve) at 12 weeks, and found a statistically significant difference of $57 \mathrm{ml}$ and $86 \mathrm{ml}$ between ipratropium and $12 \mu \mathrm{g}$ of formoterol and $24 \mu \mathrm{g}$ of formoterol, respectively (Dahl et al., 2001). However, the study also reported an a priori difference of $120 \mathrm{ml}$ as clinically relevant, which was not observed between formoterol and ipratropium. Another study of salmeterol reported change in $\mathrm{FEV}_{1} \mathrm{AUC}$ from baseline at hourly intervals, and found salmeterol to be statistically different from ipratropium at 4 and 6 hours after bronchodilator administration at the end of 12 weeks (Mahler et al., 1999).

A timed walking test is a measure of functional exercise capacity that indicates the level of dyspnea. Both salmeterol trials have found no differences between salmeterol and ipratropium in either the distance walked or the post-walk dyspnea scores using the 6 minute walking test (Mahler et al., 1999; Rennard et al., 2001). Formoterol also showed similar results on exercise capacity as measured using the shuttle walking test, with 41 percent in the formoterol group and 38 percent of patients in the ipratropium group reaching a clinically significant improvement of $\geq 30 \mathrm{~m}$ after 12 weeks (difference between groups not statistically significant) (Wadbo et al., 2002). Dyspnea scores using the Baseline Dyspnea Index (BDI) and the Transitional Dyspnea Index (TDI) did not differ significantly between salmeterol and ipratropium groups in both trials. Only one formoterol trial assessed daytime and nighttime dyspnea scores using a symptom scale, and also found insignificant differences compared with ipratropium (Wadbo et al., 2002).

Ipratropium and long-acting beta-agonists reported similar symptom scores at the end of 12 weeks in 3 trials (Mahler et al., 1999; Rennard et al., 2001; Wadbo et al., 2002). In one trial, the daily symptom score was 0.3 units lower in the formoterol group (only for $12 \mu \mathrm{g}$ and 
not $24 \mu \mathrm{g}$ ) compared to the ipratropium group that was statistically significant (Dahl et al., 2001). Increase in symptoms necessitates increased use of rescue medications, measured as daily number of puffs or inhalations. Therefore, the trials that showed no differences in symptom scores also showed no differences in daily number of puffs or inhalations of rescue medication required (Mahler et al., 1999; Rennard et al., 2001; Wadbo et al., 2002), while the formoterol trial showed significant differences (Dahl et al., 2001). Dahl et al. showed the mean daily number of puffs of inhaled albuterol during the treatment period to be $1.2,1.7$, 2.5, and 2.0 in the $12 \mu \mathrm{g}, 24 \mu \mathrm{g}$ formoterol, ipratropium and placebo group, respectively (Dahl et al., 2001). Both doses of formoterol produced a significant reduction in the need for rescue medication versus ipratropium $(\mathrm{p} \leq 0.014)$.

In terms of HRQol, no differences were seen in the total Chronic Respiratory Questionnaire (CRQ) score between salmeterol and ipratropium (Mahler et al., 1999; Rennard et al., 2001). Formoterol again showed inconsistent results on the St. George's Respiratory Questionnaire (SGRQ). The trial conducted by Dahl et al. found that formoterol differed statistically from ipratropium by -3.79 units on total SGRQ score, less than the minimum clinically important difference of four units (Dahl et al., 2001). The other formoterol trial reported no statistically significant differences between groups (Wadbo et al., 2002).

\section{Observational Studies:}

Two observational studies have compared the use of salmeterol relative to ipratropium on exacerbations requiring hospitalization, using a similar cohort study design, but different databases. Five therapy cohorts were compared based on initiation with an index prescription during a defined period: ipratropium or combination of ipratropium and albuterol, salmeterol alone, inhaled corticosteroid alone, combination of inhaled corticosteroid and ipratropium, and combination of inhaled corticosteroid and salmeterol. The presence of combination therapy was based on patterns of medication utilization defined in the first 60 days after the index date. The first study used the Pharmetrics database, which is a collection of managed care medical and prescription claims. The study found that the use of salmeterol alone did not significantly impact the risk of a COPD-related hospitalization compared to ipratropium (HR: 0.82; 95\% CI: 0.59 to 1.13) (Burney et al., 
2003). The second study using a state Medicaid database reported similar findings (HR: 0.76; $95 \%$ CI: 0.54 to 1.06 ) (Rascati et al., 2005).

\section{Inhaled Corticosteroids}

The decline in $\mathrm{FEV}_{1}$ will be discussed for ICS as the impact on this outcome affects recommendations for use of these drugs in COPD, and is important to understanding the impact of these drugs on mortality (Pauwels et al., 2001).

\section{Annual Decline in $\mathrm{FEV}_{1}$}

The rate of decline in $\mathrm{FEV}_{1}$ indicates progression of the disease, and is usually represented as a negative number in $\mathrm{ml} /$ year. A treatment effect is defined as a unit difference in the annual rate of decline between groups. Therefore, when comparing a treatment to a placebo group a positive difference is evidence of a treatment effect. Almost all studies measured rate of decline in $\mathrm{FEV}_{1}$ for each treatment group in terms of an $\mathrm{FEV}_{1}$ slope. This slope was obtained by averaging individual regression coefficients of a model regressing $\mathrm{FEV}_{1}$ on time for each individual in the study. Thus, a treatment effect for each study is determined by calculating a difference in $\mathrm{FEV}_{1}$ slope between treatment groups. Clinical Trials and Meta-Analyses:

Two long-term, uncontrolled studies showed that systemic corticosteroids slowed the progression of decline in lung function in patients with moderate-to-severe COPD. These preliminary findings prompted the scientific community to determine the role of ICS for COPD, since the inhaled route has a lower probability for long-term side-effects compared to the systemic route. A number of long-term clinical trials have since then been conducted to determine the impact of ICS on lung function decline. Earlier trials generally included asthma patients, did not conduct an intent-to-treat analysis, or lacked sufficient power (Dompeling et al., 1993; Rutten-van Molken, Van Doorslaer, Jansen, Kerstjens, \& Rutten, 1995; Weir et al., 1999; Renkema et al., 1996). To elucidate the impact of ICS in COPD, it was important to avoid confounding by the beneficial effect of ICS in asthma. Thus, clinical trials conducted later, specifically used criteria to exclude patients with asthma based on a diagnosis, evidence of atopy, or significant bronchodilator or steroid reversibility. 
A meta-analysis of three earlier trials with duration of at least 2 years, re-analyzed the original clinical data of these trials for patients with COPD without evidence of asthma, who had a mean $\mathrm{FEV}_{1}$ of $46 \%$ predicted ( $\mathrm{n}=183=1 / 3 \mathrm{rd}$ of the total) (van Grunsven et al., 1999). Results of the meta-analysis showed that treatment with ICS resulted in a difference of $+0.0341 /$ year in the annual rate of decline in pre-bronchodilator $\mathrm{FEV}_{1}(\mathrm{p}=0.026)$. When the treatment effect was analyzed by dose of inhaled corticosteroid, the estimate was +0.002 $1 /$ year (NS) for the low dose $(800 \mu \mathrm{g} /$ day $)$ and $+0.0391 /$ year $(\mathrm{p}=0.043)$ for the high dose $(1500 \mu \mathrm{g} /$ day $-1600 \mu \mathrm{g} /$ day $)$. It should be noted that the sample size for the low-dose group was only eight.

Following these preliminary findings, several major clinical trials have investigated the impact of ICS on annual rate of decline in $\mathrm{FEV}_{1}$ in COPD-only patients (Vestbo et al., 1999; Pauwels et al., 1999; Lung Health Study Group, 2000; Burge et al., 2000; Paggiaro et al., 1998; Bourbeau et al., 1998). All of these trials showed a trend toward a lower rate of annual decline in $\mathrm{FEV}_{1}$ among the treatment groups. None of these differences, which ranged from a minimum of $2.8 \mathrm{ml} /$ year to a maximum of $36.3 \mathrm{ml} /$ year, achieved significance. Even though a majority of the trials that included only patients with COPD without asthma showed that use of inhaled corticosteroid did not significantly impact the rate of $\mathrm{FEV}_{1}$ decline, two meta-analyses pooling data from these trials reached opposite conclusions (Highland et al., 2003; Sutherland et al., 2003). A meta-analysis by Highland et al. pooled data from studies by Renkema et al., Weir et al., the Copenhagen study, the European Respiratory Society Study on COPD (EUROSCOP), the Lung Health Study (LHS), and the Inhaled Steroids in Obstructive Lung Disease (ISOLDE) study (Highland et al., 2003). Data on a total of 3,571 patients were included (1,784 and 1,787 in the treatment and placebo groups, respectively). A fixed-effects model was used to combine treatment effects from each study, and the variance of each study was taken into account by weighting the pooled estimate of the treatment effect. The pooled estimate for differences in $\mathrm{FEV}_{1}$ decline between groups was found to be $+5.31 \pm 3.0 \mathrm{ml} /$ year $(95 \% \mathrm{CI}:-1.2$ to $11.2 \mathrm{ml} /$ year) showing a statistically and clinically insignificant impact of ICS on progression of COPD. In addition, the study results showed that the baseline $\mathrm{FEV}_{1}$ did not impact the significance or magnitude of treatment effect. Thus, those that had a baseline $\mathrm{FEV}_{1}<51 \%$ predicted showed a treatment effect of $+11 \mathrm{ml} /$ year (95\% CI: -1.0 to $23.1 \mathrm{ml} /$ year), whereas those with baseline $\mathrm{FEV}_{1} \geq 51 \%$ 
predicted showed a treatment effect of $+2.9 \mathrm{ml} /$ year $(95 \% \mathrm{CI}:-4.3$ to $10.1 \mathrm{ml} / \mathrm{year})$. This is

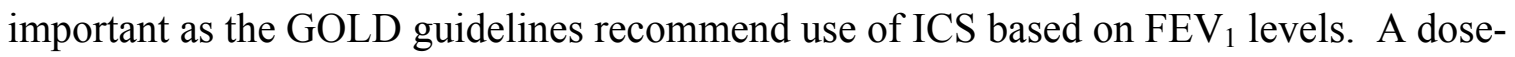
response effect was not analyzed in this meta-analysis.

Five studies included in the meta-analysis by Highland et al. were also included in the second meta-analysis by Sutherland et al. (Sutherland et al., 2003). A sixth study included in the latter was the data from the meta-analysis conducted by van Grunsven et al. (van Grunsven et al., 1999) which included the Renkema et al. study (Renkema et al., 1996). Thus, this meta-analysis actually included data from subjects enrolled in eight individual trials. A total of 3,715 patients were included in this study. Using a random-effects model and in contrast to almost all trials that were included, a significant difference of $+7.7 \mathrm{ml} /$ year (95\% CI: 1.3 to $14.2 \mathrm{ml} /$ year) was found, the clinical significance of which is questionable. Although an explicit dose-response analysis was not conducted, a meta-analysis of trials with high-dose steroid regimens showed a greater reduction of $+9.9 \mathrm{ml} /$ year (95\% CI: 2.3 to 17.5 $\mathrm{ml} /$ year). Similar to the findings by Highland et al. (Highland et al., 2003), a non-significant reduction in the rate of $\mathrm{FEV}_{1}$ decline of $18.3 \mathrm{ml} /$ year $(95 \% \mathrm{CI}$ : -1.5 to $38.0 \mathrm{ml} /$ year) was seen for subjects with a baseline $\mathrm{FEV}_{1} \leq 50 \%$ predicted.

\section{Mortality}

\section{Clinical Trials and Meta-Analyses:}

Four major clinical trials have assessed the impact of ICS on mortality (Vestbo et al., 1999; Pauwels et al., 1999; Burge et al., 2000; Lung Health Study Group, 2000). All four trials showed a trend for reduced risk of mortality; however none found a significant effect. The primary outcome of all these trials was the annual rate of $\mathrm{FEV}_{1}$ decline. None conducted a power analysis to determine if they had sufficient power to detect a difference in the mortality outcome. A meta-analysis by Alsaeedi et al. combined data from these trials, and similarly found an overall trend for decreased risk of 0.84 (95\% CI: 0.6 to 1.18 ) (Alsaeedi et al., 2002). Another scientific review pooled mortality data and found an insignificant reduced risk for mortality of 0.78 (95\%CI: 0.58 to 1.05$)$ (Sin et al., 2003c).

\section{Observational Studies:}

In contrast to the above evidence, a few observational studies have found ICS to reduce mortality by 25 percent to almost 75 percent. One of the first observational studies by Sin 
and Tu analyzed data on hospital discharges, ER and outpatient visits, and prescription medications for residents of Ontario to determine the relationship between use of ICS and mortality in elderly patients with COPD who were recently hospitalized for their disease (Sin et al., 2001). A longitudinal cohort study design was used in which the exposed and nonexposed groups were defined as those being prescribed or not being prescribed ICS within 90 days of discharge from an index hospitalization, respectively. Follow-up began on the date of discharge of the index hospitalization and continued till death occurred. The study results showed that after adjusting for relevant covariates, patients who received ICS had a 29 percent lower risk of death compared to the control group. One of the main limitations of the study was that the follow-up started from the date of discharge of the index admission. Thus, although events were counted from this date for the non-exposed group, events for the exposed group were counted from the date of receipt of the prescription of inhaled corticosteroid. This aspect of the study design creates a particular kind of bias called immortal time bias which basically refers to the creation of an immortal time period for the exposed group. This period is created as a result of the definition of exposure status, and is termed immortal because no events can be counted for the exposed group during this time period. This bias has the effect of increasing the number of events counted in the nonexposed group, thereby under-estimating the risk ratio. This bias was exemplified using the same study design but with another database by Suissa et al. (Suissa, 2003). In response to Suissa's analysis, the authors addressed the impact of immortal time bias by excluding all who died within the first 90 days of discharge. The results did not change with relative risk estimates for all-cause mortality 0.79 (95\% CI: 0.71-0.88).

Sin et al. also analyzed the impact of varying doses (low, medium and high) of ICS on mortality over a 3 year follow-up period from the date of an index hospitalization (Sin et al., 2003a). In this study design, the exposure was defined as having any prescription for an ICS anytime from the date of discharge of the index hospitalization to the end of follow-up (date of death or 3 years). A daily dose was computed using the total amount dispensed for the first prescription and the period of time elapsed between the first two prescriptions. Since all ICS were considered, the daily dose was calculated in terms of beclomethasone equivalents. The cohort was then categorized into five mutually exclusive categories: not dispensed, low $(\leq 500 \mu \mathrm{g} /$ day $)$, medium $(501-1000 \mu \mathrm{g} /$ day $)$, high $(>1000 \mu \mathrm{g} /$ day $)$, and indeterminate dose. 
Similar to the previous analysis, therapy with ICS showed a 25 percent reduction in all-cause mortality rate. When analyzed by dose level, those on low-dose, medium-dose or high-dose experienced a 23 percent, 52 percent and 45 percent reduction in all-cause mortality compared to those who did not receive any ICS. The authors tried to address immortal time bias by performing a subgroup analysis excluding all patients who had less than 90 days, 6, 9 and 12 months of follow-up, and hence a lower probability of receiving ICS. However, in this case, the follow-up time was 3 years. Thus, there is a slight possibility that those who were categorized as not receiving ICS were classified as such because they died. This has the impact of increasing the events in the non-exposed group and a consequent treatment effect for the exposed group.

Another group of researchers using the UK General Practice Research Database (GPRD) assessed the impact of fluticasone, an ICS, on mortality (Soriano et al., 2002). A cohort study design was used by defining drug exposure on an intent-to-treat basis. Thus, users of fluticasone were physician-diagnosed patients with COPD who received at least three prescriptions for these drugs over an initial 6-month period. The reference group comprised patients who had not received ICS since diagnosed with COPD, but had at least three prescriptions for other COPD-related drugs over an initial 6-month period. To address the issue of immortal time bias, patient follow-up time only started a day after the 6-month period during which exposure was assessed and continued till the patient died or 3 years were complete. The adjusted survival analyses showed that the use of fluticasone alone resulted in a 38 percent reduction in mortality risk over a 3 year period compared to the reference group. An additional analysis comparing the use of any ICS demonstrated a class effect with an adjusted survival rate of 77.9 percent. A secondary objective of the study was to assess if a dose-dependent effect on survival could be demonstrated. To assess this objective, a nested case-control study design was used. The total number of prescriptions during the entire period of follow-up was used to calculate the cumulative amount of exposure to each treatment type, and then assess a mean annual exposure to treatment. The results of the doseresponse analysis mirrored the results of the cohort study design. The use of each additional annual prescription of fluticasone significantly reduced the risk of death by 13 percent. These same authors further conducted another analysis using the study design by Sin et al. to assess the use of ICS on death or re-hospitalization (Soriano et al., 2003). Thus, exposure 
was defined as use of at least one prescription for an ICS within 90 days after a first (index) hospitalization for a COPD-related condition. Follow-up was assessed from the date of discharge of the index hospitalization to a maximum of 1 year after this date or till death or re-hospitalization occurred. In this study, immortal time bias was addressed by restricting the analyses to patients who survived for a period of 30 days after the index hospitalization. Adjusted survival analyses showed that the use of ICS alone reduced the risk of rehospitalization or death by 16 percent. A separate risk ratio for death was not computed.

Two more studies assessing the impact of ICS on mortality were conducted. The results of these two studies were not formally published but were published as part of a paper from a symposium discussing the issues of observational studies in COPD (Burney et al., 2003). Therefore, details of the study designs are not available, and only certain results are mentioned. One study was conducted by researchers in the Netherlands using data from the Integrated Primary Care Information (IPCI) database. A similar cohort design used in the study by Soriano et al. (Soriano et al., 2002) was used where exposure was defined as having at least two prescriptions of fluticasone after a COPD diagnosis. To ensure that patients had at least moderate-to-severe COPD, patients 50 years of age and older were required to have at least two bronchodilators. Study results showed that exposure to fluticasone significantly reduced the risk for mortality by 66 percent (Hazards ratio $(\mathrm{HR})=0.34 ; 95 \% \mathrm{CI}$ : 0.18 to 0.66), a risk reduction more than twice as high than that seen in the study by Soriano et al. (Soriano et al., 2003; Soriano et al., 2002). The other study in the U.S. used administrative databases from two health maintenance organizations (HMO). A cohort design was used where a person was considered exposed if they had at least 90 days (not necessarily consecutive) of prescription fills. Study results showed a significant survival advantage for exposure to ICS $(\mathrm{HR}=0.59 ; \mathrm{p}=0.0001)$. However, the impact of ICS alone on mortality was no longer significant when the cohort was restricted to those without a prior diagnosis of asthma $(\mathrm{HR}=0.74 ; \mathrm{p}=0.1)$.

Unlike the observational studies described above, and similar to the findings of randomized controlled trials, two studies did not find a protective effect of ICS on mortality. These studies used specific study designs or analytical techniques that would account for immortal time bias. The first study by Suissa et al. was described earlier (Suissa, 2003). Another study by Fan et al. used medical and prescription claims data from a Veterans 
Affairs health system (Fan et al., 2003). The patients were identified if they had an outpatient clinic visit or a hospitalization with a primary or secondary diagnosis of COPD between February 1997 and December 1999. This visit date was then considered the index date, and defined their time of entry into the cohort. To ensure that these were patients with COPD, only those over 45 years of age and with at least one pulmonary medication in the 90 day period before the index visit were included. Study subjects were required to have a minimum of a year of follow-up to assess the outcome of death. A cohort study design was used using three methods to define exposure to ICS depending on timing of drug use. In all three methods, a person was considered exposed if prescriptions were filled for more than 80 percent of days during a defined time interval of 90 days. Thus, exposure was defined in terms of baseline use, average use and recent use. Immortal time bias was accounted by using the concept of a 90-day interval before event occurrence to determine exposure status. Survival analysis using time-dependent methods showed that exposure to ICS was not associated with a decrease in mortality, regardless of the dose of ICS used (low/high). Although the rate ratios were less than 1 showing a trend towards decreased mortality, a null effect was found with confidence intervals including 1 . The timing of drug use (baseline, average, or recent) did not impact the magnitude or direction of the risk ratios.

\section{Exacerbations}

\section{Clinical Trials and Meta-Analyses:}

The meta-analysis by van Grunsven et al. found ICS to modify the rate of $\mathrm{FEV}_{1}$ decline but did not find any beneficial effect on the rate of exacerbations. The treatment group had 0.9 exacerbations per year and the placebo group had 1.0 exacerbation per year (van Grunsven et al., 1999). They attributed the lack of effect to differences in definitions of exacerbations among the included trials. Alsaeedi et al. conducted a meta-analysis by combining data from six recent randomized, placebo-controlled trials of at least six months duration using the DerSimonian-Laird random-effects model. Of the six trials, two showed no reduction in risk (Vestbo et al., 1999; Bourbeau et al., 1998) while four showed statistically significant effects (Burge et al., 2000; Paggiaro et al., 1998; Weir et al., 1999; Lung Health Study Group, 2000) as calculated from data in the published studies by the authors. Of the four studies that showed an effect in the meta-analysis, published articles of 
two of the trials did not show any differences in exacerbations. One of the trials showed a trend towards decreased exacerbations that was not statistically significant (Weir et al., 1999). The primary reason was that the sample size was very low $(n=98)$. For the second trial, the meta-analysis defined exacerbations as hospitalization for respiratory conditions, and calculated a significant risk reduction of 0.46 (95\% CI: 0.26 to 0.80$)$. However, the published article of this trial did not clearly define nor specify exacerbation as an outcome measure (Lung Health Study Group, 2000). This article reported that there were no significant differences between the treatment groups in unscheduled visits, ER visits, or hospitalizations.

Like the meta-analysis by van Grunsven et al., the definitions of exacerbations also differed among all the included trials (Alsaeedi et al., 2002) in the meta-analysis by Alsaeedi et al. However, the latter showed that the use of ICS led to a 30 percent reduction in exacerbations with a relative risk $=0.70(95 \% \mathrm{CI}: 0.58$ to 0.84$)$. The test for heterogeneity was significant $(\mathrm{p}=0.03)$, indicating that the effects varied among studies. The Copenhagen City Lung Study was primarily responsible for the heterogeneity, and reported a risk ratio of 0.96 (95\%CI: 0.77 to 1.20 ) (Vestbo et al., 1999). This may have been due to the study definition of an exacerbation (more cough or phlegm than usual instead of worsening of symptoms with the need for additional medication). Exclusion of this study further lowered the relative risk to 0.67 (95\% CI: 0.63 to 0.71 ) without evidence of heterogeneity. A doseresponse effect could not be demonstrated because of the similarity in doses used in the trials, and neither was a separate analysis done based on disease severity. Another recent scientific review (Sin et al., 2003c) by some of the same authors who conducted the above metaanalysis, combined data from all six trials included in the above meta-analysis as well as an additional study (van, V, Monninkhof, van der, Zielhuis, \& van Herwaarden, 2002) published in 2002. Summary effect estimates of the review showed a 24 percent reduction in exacerbations with a risk ratio of 0.76 (95\% CI: 0.72 to 0.80$)$.

The lack of effect in the Copenhagen study (Vestbo et al., 1999) has also been attributed to the mild disease severity level of the clinical trial population. This led to the hypothesis that the beneficial effect of ICS may be modified by disease severity. Evidence from two sources reveals that ICS significantly reduced the risk for exacerbations in those with moderate-to-severe COPD compared to patients with mild COPD. A secondary analysis of 
the ISOLDE clinical trial data showed that the total exacerbation rate differed significantly between the fluticasone and placebo group (1.47/year vs. 1.75/year) in patients with moderate-to-severe COPD $\left(<50 \% \mathrm{FEV}_{1}\right.$ predicted) (Jones, Willits, Burge, \& Calverley, 2003). On the other hand, patients with mild COPD with $\geq 50 \% \mathrm{FEV}_{1}$ predicted showed no difference in total exacerbation rate (fluticasone: 0.67/year and placebo: 0.92/year). Although not an explicit comparison, the scientific review mentioned above (Sin et al., 2003c) noted that the Copenhagen City Lung study (Vestbo et al., 1999), which had the highest mean $\mathrm{FEV}_{1}$ value (2.38L), failed to demonstrate a beneficial effect of ICS on exacerbation rate compared to the other studies which had a mean $\mathrm{FEV}_{1}<70 \%$ predicted.

Data from the clinical trials that have demonstrated a beneficial impact of ICS on exacerbations also showed that this impact was more prominent for moderate to severe than mild exacerbations. A six-month trial conducted by Paggiaro et al. (Paggiaro et al., 1998) that assessed the effect of fluticasone propionate on the rate of exacerbations, showed no significant differences in the total number of patients who had at least one exacerbation or the total number of exacerbations. However, a significantly higher proportion of patients in the placebo (86\%) compared to the fluticasone group (60\%) experienced a moderate-tosevere exacerbation. Similar to the trial by Paggiaro et al., the proportion of patients in the ISOLDE trial experiencing at least one exacerbation was not different between the treatment groups (Burge et al., 2000). However, among patients experiencing moderate exacerbations (exacerbations requiring oral corticosteroids), the number of patients was reduced by 50 percent in those treated with fluticasone compared to placebo, regardless of the underlying COPD severity. Thus, results from these studies suggest that ICS may significantly impact the severity of the exacerbation but not the overall frequency.

Discontinuation of ICS therapy has been shown to be detrimental for patients with COPD as seen in a recent clinical trial called COPE. The COPE study enrolled patients from an outpatient pulmonary clinic to assess the impact of discontinuing maintenance therapy with inhaled fluticasone (van, $V$ et al., 2002). In this study, 244 patients were treated with inhaled fluticasone $(1000 \mu \mathrm{g} / \mathrm{day})$ for four months. After this phase, 123 were randomized to continue and 121 to discontinue with their fluticasone therapy for 6 months. Unlike the previous studies, where difference in the exacerbation rate was assessed, the outcome in this study was risk of a first and second exacerbation after discontinuation. A hazard ratio of 1.5 
( $95 \%$ CI: 1.05 to 2.1 ) for a first exacerbation and of 2.4 (95\% CI: 1.5 to 3.9 ) for a second exacerbation in favor of the fluticasone group was found. Also, 21.5 percent of patients in the placebo group experienced recurrent exacerbations compared to 4.9 percent in the fluticasone group. Effect modification based on disease severity was also seen in this study with the hazard ratio of a first exacerbation for those who discontinue fluticasone being 2.1 (95\% CI: 1.1 to 3.6$)$ among patients with $\mathrm{FEV}_{1}<50 \%$ predicted compared to $1.2(95 \% \mathrm{CI}$ : 0.8 to 2.0 ) among those $\geq 50 \%$ predicted.

\section{Observational Studies:}

Observational studies have provided conflicting evidence regarding the impact of ICS on the risk of exacerbations. Some of the observational studies that assessed the impact of ICS on death discussed in the previous section have also assessed the impact on exacerbations. Four studies focused on exacerbations that require hospitalizations, and found a significant effect. Similar to their findings on mortality, Sin et al. (Sin et al., 2001) and Soriano et al. (Soriano et al., 2003) found that ICS reduced the risk of an exacerbation requiring a hospitalization by 24 percent and 16 percent, respectively. Another study attempted to compare hospitalization risk of various initial treatment regimens using the Pharmetrics database (Burney et al., 2003). The study results showed that compared to the ipratropium therapy cohort, patients treated with ICS alone reduced the risk for a COPD-related hospitalization in the 12-month follow-up period by 29 percent. Analysis of a state Medicaid database using a similar cohort design as the Pharmetrics study found a 16 percent reduction in risk of hospitalization that was not statistically significant (HR:0.84; $95 \%$ CI: 0.69 to 1.03 ) (Rascati et al., 2005).

Two observational studies did not find a significant impact of ICS on exacerbations. Fan et al. found an insignificant effect of ICS on exacerbations requiring hospitalization (Fan et al., 2003). Bourbeau et al. analyzed administrative data of the Saskatechewan universal healthcare system using a nested case-control study design (Bourbeau et al., 2003), and reached the same conclusion as Fan et al. A nested case-control uses the case-control analytical technique within a cohort of patients. The cohort in this study included patients with COPD on regular therapy with COPD-related drugs and those with a first hospitalization with a primary diagnosis of COPD, ensuring a moderate-to-severe subgroup of patients with COPD. The outcome of interest was a re-hospitalization for COPD, and thus 
the case patients were defined as those who were re-hospitalized with a primary diagnosis of COPD. Use of ICS was defined in relation to the timing of the outcome (re-hospitalization) and in terms of dose. Thus, any use was defined as the dispensing of one or more prescriptions in the year prior to the index date. Current use was defined as a prescription being dispensed within 60 and 30 days of the index date for the high-dose and low-dose formulations, respectively. Past use was defined as the exposure for subjects with any use but not current use. In addition, similar to the dose-response analysis by Sin et al., a mean daily dose was calculated in the prior year, and categorized as $\leq 400 \mu \mathrm{g} / \mathrm{day}, 401-800 \mu \mathrm{g} / \mathrm{day}$, and $>800 \mu \mathrm{g} /$ day. Conditional logistic regression analysis revealed that exposure to ICS defined as any, current or past use did not result in any benefit in preventing exacerbations requiring hospitalizations. This result is in direct contrast to the afore-mentioned studies, and does not support findings from a few randomized controlled trials of ICS (Burge et al., 2000; Paggiaro et al., 1998).

Acute exacerbations in COPD are defined as an increase in symptoms of COPD that often require a change in regular medication. These exacerbations, regardless of whether they require hospitalization, are important since they impact morbidity and quality of life (Seemungal et al., 1998; Donaldson et al., 2002). Only two studies assessed the impact of ICS on exacerbations that do not require hospitalization, and found no effect. A study conducted by de Melo et al. using the Saskatechewan database, assessed the impact of ICS on the rate of a first acute exacerbation (de Melo et al., 2004a). Thus, a cohort of patients with COPD was defined by selecting individuals $\geq 55$ years who had received at least three prescriptions for COPD-related medications during a year, between January 1, 1990 and December 31, 1997, and who had not experienced an acute exacerbation before this criteria was met. On cohort entry, individuals were followed from the date of the third prescription to the date of a first exacerbation, death, emigration from the province, or December 31 , 1999 to allow for a minimum follow-up of 2 years. The first occurrence of an acute exacerbation was chosen as the outcome of interest since recurring exacerbations cause a persistent deterioration in overall well-being, and hence it was felt important to determine if ICS could delay the time to a first acute exacerbation. An acute exacerbation was defined in terms of the extent of healthcare use based on the availability of data. A moderate exacerbation was defined by prescriptions for a systemic antibiotic and an oral corticosteroid 
on the same day, whereas a severe exacerbation was defined as a hospitalization with a primary discharge diagnosis of COPD. A nested case-control design was used where cases were defined as those who experienced an acute exacerbation. Use of ICS was defined similar to the study by Bourbeau et al. in terms of any use, current use, and past use. The only difference was that a prodromic phase of a COPD exacerbation was considered as a period of 15 days before the occurrence of an exacerbation during which dispensing of an ICS could occur. This period was excluded for defining any and past use, while current use was analyzed by including and excluding this period. Results of the conditional logistic regression analysis showed that exposure to ICS defined in terms of any use, current use and past use exhibited an increase in the risk for a first exacerbation. Thus, rate ratios ranged from 1.27 to 1.52 to describe the increased risk of a first exacerbation due to ICS.

Similar to these findings, Fan et al. also assessed the effect of ICS on exacerbations not requiring a hospitalization and found a null effect. In contrast to the definition used by de Melo et al. (de Melo et al., 2004a), Fan et al. (Fan et al., 2003) defined moderate exacerbations as outpatient visits with a primary ICD-9 code for COPD combined with a new prescription for a 14 (or fewer) day course of either prednisone or a commonly used antibiotic (amoxicillin, sulfa drugs, cephalosporins, quinolones, tetracyclines, and macrolides) that was dispensed and filled within 24-48 hours of the clinic visit.

\section{Combination of Inhaled Corticosteroid and a Long-Acting Beta-Agonist}

Long-acting beta-agonists and ICS have different mechanisms of action, and target different aspects of the pathophysiologic processes in COPD. Therefore, it is hypothesized that their combination can be expected to have a synergistic or additive effect. Molecular evidence of this interaction between the drug classes shows that corticosteroids increase beta2 receptor synthesis through which long-acting beta-agonists exert their action, and longacting beta-agonists prime the glucocorticoid receptor for steroid-dependent activation and nuclear translocation (Sin et al., 2004). Two combination products have been evaluated in

clinical trials. One combination product consisting of the long-acting beta-agonist salmeterol and the ICS, fluticasone has recently been approved by the Food and Drug Administration (FDA) for COPD. The other combination product, not yet approved by the FDA for COPD, includes the long-acting beta-agonist formoterol and the ICS, budesonide. At least four 
randomized, clinical trials have been conducted to determine if the interaction effect of this combination translates into important outcomes such as reductions in exacerbations and mortality. Some of the observational studies discussed in the prior section analyzing the effectiveness of ICS, have also assessed the impact of the combination of ICS and longacting beta-agonists. Therefore, the study designs will not be reiterated in this section, and only results of the combination therapy will be detailed.

\section{Exacerbations}

\section{Clinical Trials and Meta-Analyses:}

Three randomized clinical trials that have assessed the impact of combination therapy on the rate of exacerbations have found a significant reduction compared to placebo therapy, with reductions ranging from 25 percent to 30 percent (Calverley et al., 2003a; Calverley et al., 2003b; Szafranski et al., 2003). Moderate exacerbations were defined as worsening of symptoms requiring treatment with antibiotics and/or oral corticosteroids, while severe exacerbations were generally those requiring hospitalization for respiratory conditions. Only one study found that combination therapy also significantly reduced the risk for mild exacerbations (Szafranski et al., 2003). They defined mild exacerbations as days with at least four inhalations of reliever medications above the mean use, and were calculated from daily diary cards. These trials also individually assessed the impact of combination therapy on exacerbations requiring only oral corticosteroid courses, and found risk reductions of higher magnitude ranging from 31 percent to 45 percent compared to the risk reduction for overall exacerbations (Szafranski et al., 2003; Calverley et al., 2003a; Calverley et al., 2003b).

Three meta-analyses pooled data from these trials. Sin et al. pooled data from two trials that assessed the impact of the combination of budesonide/formoterol and another of fluticasone/salmeterol on exacerbations, and found that combination therapy reduced the risk by 30 percent ( $\mathrm{RR}=0.7,95 \% \mathrm{CI}: 0.62$ to 0.78 ) (Sin et al., 2003c). A second meta-analysis conducted later by Sin et al. pooled data from four trials, including those from the previous analysis (Sin et al., 2004). However, one of the trials (Mahler et al., 2002) that was included in estimating the pooled risk ratios of reduced exacerbations withdrew patients if they experienced more than one exacerbation resulting in hospitalization or treated with an inhaled or oral corticosteroid. This criterion could reduce estimation of any treatment effect. 
It is unclear how the inclusion of this study affected the pooled risk ratio of exacerbations. However, the proportions withdrawn due to exacerbation were similar across treatment groups in that trial (Mahler et al., 2002). This meta-analysis showed that the combination of ICS and long-acting beta-agonists resulted in a 48 percent reduction in exacerbations $(95 \%$ CI: $60 \%$ to $32 \%$ ). Unlike the other meta-analyses discussed so far, a Cochrane review pooled data from three clinical trials (Calverley et al., 2003a; Calverley et al., 2003b; Szafranski et al., 2003) that had similar definitions of exacerbations, and found a significantly reduced risk of 24 percent for combination therapy compared to placebo ( $\mathrm{RR}=0.76$; 95\% CI: 0.68 to 0.84 ) (Nannini et al., 2003). Thus, magnitude of risk reduction of 25 percent to 30 percent for moderate to severe exacerbation from two meta-analyses mirror that obtained in the original trials for combination therapy of ICS and long-acting betaagonists.

It is also important to know the effectiveness of the combination compared to the monotherapy components to determine any additive or synergistic effects. Most of the data from the clinical trials showed no difference in reducing exacerbations between the combination therapy and the ICS component. Only one trial reported a significant risk reduction of 22.7 percent for exacerbations for the combination therapy when compared to ICS monotherapy (Szafranski et al., 2003). Pooled risk ratios from two meta-analyses, showed no difference in the rate of exacerbations. Similar magnitudes of risk ratios were noted in these studies; Sin et al. (Sin et al., 2003c): $R R=0.90$ (95\% CI: 0.80 to 1.02) and Cochrane review (Nannini et al., 2003): RR=0.91 (95\% CI: 0.81 to 1.02). Another metaanalysis by Sin et al. however, found a significantly lower risk for exacerbations compared to that of ICS alone ( $R R=0.74 ; 95 \%$ CI: 0.55 to 0.99 ) (Sin et al., 2004).

The data comparing the combination therapy to the long-acting beta-agonist component however, shows significant differences. Two trials found that combination therapy (both were budesonide/formoterol) had a significant risk reduction of 23 percent to 31 percent compared to the long-acting beta-agonist (Szafranski et al., 2003; Calverley et al., 2003b). Results from all three meta-analysis showed that combination therapy was significantly better compared to long-acting beta-agonist alone. Pooled risk ratios reported were: Sin et al. (Sin et al., 2003c), 0.8 (95\% CI: 0.71 to 0.90); Sin et al. (Sin et al., 2004), 0.67 (95\% CI: 0.51 to 0.89 ), and the Cochrane review (Nannini et al., 2003), 0.85 (95\% CI: 0.77 to 0.95 ). 


\section{Observational Studies:}

Observational studies have assessed the impact of combination therapy on only severe exacerbations requiring hospitalizations, and not moderate exacerbations. One study by Soriano et al. assessed the impact of receiving a prescription for a combination therapy of ICS and long-acting beta-agonist within 90 days of a hospitalization on a combined outcome of re-hospitalization or death (Note: Separate risk ratios for these outcomes were not reported). A 41 percent reduction in risk was found for this dual outcome (Soriano et al., 2003). Two studies using similar cohort designs compared hospitalization risk of various initial treatment regimens (Burney et al., 2003) relative to ipratropium. One study used the Pharmetrics database, which is a collection of medical and prescription claims from several managed care plans (Burney et al., 2003). The study results showed that compared to the ipratropium therapy cohort, patients with the combination of ICS and salmeterol had the greatest risk reduction (74\%) in the 12-month follow-up period. The other study used a state Medicaid database (Rascati et al., 2005), and found that the combination of ICS with salmeterol had a significant risk reduction of 35 percent $(\mathrm{HR}=0.653 ; 95 \% \mathrm{CI}=0.43$ to 0.99$)$ similar in magnitude to the study by Soriano et al. (Soriano et al., 2003). As can be noted, this risk reduction was borderline significant. It is not clear why the magnitude of the risk reduction seen in the Pharmetrics study was almost twice as high compared to that seen in clinical trials and the other observational studies.

The observational studies do not provide results comparing the combination therapy with ICS monotherapy or long-acting beta-agonist monotherapy. However, the presence of any additive or synergistic effects can be ascertained by the magnitude of risk reduction relative to the reference group using only short-acting bronchodilators. Two of the three observational studies mentioned above found a significant risk reduction for the combination therapy and ICS monotherapy compared to the reference group using only short-acting bronchodilators. The risk reduction for combined outcome of re-hospitalization or death relative to a reference group using short-acting bronchodilators in the Soriano study for the combination therapy and ICS monotherapy was 41 percent and 16 percent (Soriano et al., 2003). The Pharmetrics study also reported a higher risk reduction for the combination therapy than that obtained with ICS monotherapy compared to ipratropium (74\% vs. 29\%, respectively) (Burney et al., 2003). The Medicaid analysis found a significant risk reduction 
for the combination therapy, but an insignificant risk reduction of 16 percent for ICS monotherapy compared to ipratropium (Rascati et al., 2005).

All the three observational studies found insignificant risk reductions for the long-acting beta-agonist monotherapy (salmeterol) compared to the respective reference group. In the study by Soriano et al., an insignificant effect was observed for salmeterol and a significant effect for the combination therapy on the dual outcome of death or hospitalization compared to a reference group using short-acting bronchodilators (Soriano et al., 2003). Thus, the addition of ICS may be responsible for a survival advantage, where one may not be present for long-acting beta-agonist monotherapy such as salmeterol. It should be noted that the reference group in the other two observational studies was ipratropium, and the insignificant reductions in those studies may reflect the fact that long-acting beta-agonists are as effective as ipratropium (Burney et al., 2003; Rascati et al., 2005).

\section{Mortality}

Clinical Trials and Meta-Analyses:

In the second meta-analysis by Sin et al., mortality was also assessed as an outcome (Sin et al., 2004). Three of six trials assessed mortality as a secondary outcome, and did not provide information regarding the power to determine differences. However, pooling data from these trials showed a trend towards lower mortality compared to placebo. $(\mathrm{RR}=0.66$; $95 \%$ CI: 0.32 to 1.38 ). These findings are in contrast to that obtained from three observational studies that contradict clinical trial findings.

\section{Observational Studies:}

Two studies by Soriano et al. assessed the impact of combination fluticasone and salmeterol on mortality using two different study designs using the UK General Practice Research Database (GPRD) (Soriano et al., 2002; Soriano et al., 2003). One study that assessed the combined outcome of death and re-hospitalization was already discussed in the section on the impact of the combination therapy on exacerbations, and will not be repeated here (Soriano et al., 2003). The other study used physician-diagnosed patients with COPD, and defined exposure based on the receipt of at least three prescriptions during an initial sixmonth period (Soriano et al., 2002). Treatment cohorts were defined based on the type of drugs received during this period. Patients were followed after the six-month period until 
three years or until the death of the patient. The adjusted survival analyses showed that the combined use of fluticasone and salmeterol resulted in a 52 percent reduction in mortality risk over a 3 year period compared to the reference group using only short-acting bronchodilators. An additional analysis comparing the use of any ICS with salmeterol demonstrated a class effect with an adjusted survival rate of 82.1 percent. A secondary objective of the study assessed the presence of a dose-dependent effect on survival using a nested case-control design. The total number of prescriptions during the entire period of follow-up was used to calculate the cumulative amount of exposure to each treatment type, and then assess a mean annual exposure to treatment. The use of each additional annual combined prescription of fluticasone with salmeterol significantly reduced the risk of death by 13 percent.

Two more studies assessing the impact of ICS and long-acting beta-agonists on mortality were published as part of a paper from a symposium discussing the issues of observational studies in COPD (Burney et al., 2003). A cohort study using claims data from two HMOs in the U.S. defined a person using a combination of an ICS and long-acting beta-agonist as one that had a total of at least 90 days (not necessarily consecutive) of overlapping prescription fills of the two drugs, or of the combination product. Study results showed a significant survival advantage for exposure to combination therapy similar to the findings of Soriano et al. Furthermore, the magnitude of the hazard ratios for the combination therapy ranged from 0.34 to 0.11 , which suggests a 66 percent to 89 percent reduction in mortality, substantially higher compared to the Soriano et al. studies. The second study conducted by researchers in Netherlands using data from the Integrated Primary Care Information (IPCI) database, used the cohort design similar to that of Soriano et al.(Soriano et al., 2002). Exposure was defined as having at least two prescriptions of the salmeterol, fluticasone, or a combination of salmeterol and fluticasone after a COPD diagnosis. Study results showed that exposure to fluticasone and salmeterol significantly reduced the risk of mortality by 63 percent (HR=0.37; 95\% CI: 0.21 to 0.67$)$.

The combination therapy was shown to have a higher reduction in mortality risk compared to using ICS alone in both the Soriano studies and the U.S. HMO study (Soriano et al., 2003; Soriano et al., 2002; Burney et al., 2003). The IPCI study, however, reported similar reductions in risk for both therapies. In one study by Soriano et al. the use of 
combination therapy reduced the risk of death by 52 percent while using ICS monotherapy reduced the risk by 38 percent compared to the reference group using only short-acting bronchodilators (Soriano et al., 2002). The dose-response analysis also showed higher risk reduction for the combination therapy as seen in the Kaplan-Meier survival curve, however no specific values were reported. The U.S. HMO study reported a risk reduction of 66 percent and 41 percent for the combination therapy and ICS monotherapy, respectively (Burney et al., 2003). However, when the study population was restricted to those without a prior diagnosis of asthma, the combination therapy remained significant with a risk ratio of 0.11 ( $89 \%$ reduction), while that of ICS alone was no longer significant ( $\mathrm{HR}=0.74)$. The IPCI study showed a significant risk reduction of approximately 64 percent for both groups (Burney et al., 2003).

Both the cohort and the dose-response analyses in the study by Soriano et al., showed an increased trend for survival, albeit insignificant for the use of salmeterol alone compared to the reference group (Soriano et al., 2002). The U.S. HMO study was probably the only one that showed the use of salmeterol alone significantly impacted mortality $(H R=0.55 ; p=0.02)$ compared to a reference group using short-acting bronchodilators and no ICS (Burney et al., 2003). This effect however, disappeared when the study population was restricted to those without a prior diagnosis of asthma $(\mathrm{HR}=0.57 ; \mathrm{p}=0.1)$. The IPCI study did not report results for salmeterol (Burney et al., 2003). 


\section{CHAPTER 3: METHODOLOGY}

Chapter 3 outlines the methodology used for conducting the three phases of the study. The study sample, study design, and analyses are specific to each study objective, and are individually detailed for each study objective. All phases of the study however, had a common data source: paid medical and prescription claims from West Virginia and Kentucky Medicaid. A description of the data source and the data cleaning procedures is provided below. All raw claims data were manipulated through various processes in order to extract relevant information using SAS v. 9.1.3. Statistical analyses were conducted using STATA 9.0 and SPSS 13.0.

\section{DATA DESCRIPTION \& VARIABLE EXTRACTION}

\section{Data Source}

A list of raw claims data files used for the study in both states is provided in Table 6 . The Bureau for Medical Services of the West Virginia Department of Health and Human Resources (WV-DHHR) provided the data for WV Medicaid. Similarly, the Kentucky Department of Medicaid Services of the Kentucky Cabinet for Health and Family Services (KY-CHFS) provided the data for KY Medicaid. Data are available from July 1, 1997 to June 30, 2003 for WV Medicaid and from January 1, 1998 to December 31, 2003 for KY Medicaid. Three main types of files were obtained for each state: medical claims, pharmacy claims, and eligibility files. All claims data were linked by an encrypted identification number to protect recipient confidentiality. Three types of medical claims were available: UB-92, HCFA-1500 files, and Nursing home claims files. The first two types of files included first and last dates of service, International Code of Diseases-Clinical Modification

(ICD-9 CM) diagnosis codes, Current Procedural Terminology (CPT) procedure codes, claim (extract) indicator, and total amount paid. Additionally, the UB-92 file included revenue codes, and Diagnosis Related Group (DRG). The nursing home claims files only included first and last dates of service, and total amount paid. Each record of the prescription claims data included details of a drug filled such as National Drug Codes (NDC), date dispensed, quantity dispensed, days' supply, and total amount paid. The eligibility files included 
demographic information for recipients such as date of birth, gender, race, county, and dates of enrollment. In addition to these three types of files, there were three files with provider information, drug information, and procedure code information, details of which are provided in Table 6.

TABLE 6: List of raw claims data files

\begin{tabular}{|c|c|c|c|}
\hline File Name & Details & File Name & Details \\
\hline \multicolumn{2}{|c|}{ West Virginia } & \multicolumn{2}{|c|}{ Kentucky } \\
\hline UB-92 claims & $\begin{array}{l}\text { July } 1,1997 \text { to } \\
\text { June } 30,2003\end{array}$ & UB-92 claims & $\begin{array}{l}\text { January } 1,1998 \text { to } \\
\text { December } 31,2003\end{array}$ \\
\hline $\begin{array}{l}\text { HCFA-1500 } \\
\text { claims }\end{array}$ & $\begin{array}{l}\text { July } 1,1997 \text { to } \\
\text { June } 30,2003\end{array}$ & $\begin{array}{l}\text { HCFA-1500 claims \& } \\
\text { Homehealth care claims }\end{array}$ & $\begin{array}{l}\text { January } 1,1998 \text { to } \\
\text { December } 31,2003\end{array}$ \\
\hline $\begin{array}{l}\text { Nursing home } \\
\text { claims }\end{array}$ & $\begin{array}{l}\text { July } 1,1997 \text { to } \\
\text { June } 30,2003\end{array}$ & Nursing home claims & $\begin{array}{l}\text { January } 1,1998 \text { to } \\
\text { December } 31,2003\end{array}$ \\
\hline $\begin{array}{l}\text { Prescription } \\
\text { claims }\end{array}$ & $\begin{array}{l}\text { July } 1,1997 \text { to } \\
\text { June } 30,2003\end{array}$ & Prescription claims & $\begin{array}{l}\text { January } 1,1998 \text { to } \\
\text { December } 31,2003\end{array}$ \\
\hline $\begin{array}{l}\text { Medicaid } \\
\text { eligibility file }\end{array}$ & $\begin{array}{l}\text { Eligibility information } \\
\text { until June } 30,2003\end{array}$ & Medicaid eligibility file & $\begin{array}{l}\text { Eligibility information } \\
\text { until December } 31,2003\end{array}$ \\
\hline $\begin{array}{l}\text { Managed care } \\
\text { eligibility file }\end{array}$ & $\begin{array}{l}\text { Eligibility information } \\
\text { until June } 30,2003\end{array}$ & $\begin{array}{l}\text { Managed care } \\
\text { eligibility file }\end{array}$ & $\begin{array}{l}\text { Eligibility information } \\
\text { until December } 31,2003\end{array}$ \\
\hline Provider file & $\begin{array}{l}\text { Provider number } \\
\text { Provider specialty }\end{array}$ & Provider file & $\begin{array}{l}\text { Provider number } \\
\text { Provider specialty }\end{array}$ \\
\hline Procedure file & $\begin{array}{l}\text { CPT code } \\
\text { Procedure name }\end{array}$ & Procedure file & $\begin{array}{l}\text { CPT code } \\
\text { Procedure name }\end{array}$ \\
\hline Drug file & $\begin{array}{l}\text { NDC code } \\
\text { Generic code } \\
\text { Drug name }\end{array}$ & Drug file & $\begin{array}{l}\text { NDC code } \\
\text { Generic code } \\
\text { Drug name } \\
\text { Therapeutic class code } \\
\text { Therapeutic class name }\end{array}$ \\
\hline
\end{tabular}

\section{Data Cleaning}

The UB-92 claims file contain facility claims for inpatient visits, emergency visits, and outpatient encounters, whereas the HCFA-1500 claims file contain claims submitted by health care professionals for services rendered in the outpatient (physician office or hospital outpatient), emergency room, and home-healthcare settings. These two files were 
manipulated using the following algorithm to classify all claims for hospitalizations, emergency room (ER) visits, and outpatient encounters:

\section{For WV Medicaid:}

1. Hospitalization claims in the UB92 file were identified by the presence of a DRG code not equal to '000'. Also, some claims related to a hospitalization such as those of professional services e.g. surgeon's fees will be present in the HCFA1500 file. These claims were identified by using recipient identification number (ID) and date of admission information, and included in the "final hospitalization claims" file.

2. ER claims in the UB-92 file were identified by the presence of revenue codes 450459. It is important to note that not all of the claims occurring during an ER visit will have this code since other line items will have revenue codes depending on the service/procedure provided. Hence, all claims identified to be occurring for an individual on the same date as that of claims with revenue code 450-459 will be included in the final ER claims file. Similar to hospitalization scenario, all those claims occurring on the same date for an individual for an ER visit in the HCFA1500 file will be included in the final ER claims file. ER visits for which no claim exists in the UB92 file, but claims exist in the HCFA1500 file, were identified by the presence of CPT procedure codes 99281-99285, 99288, and all claims occurring on the dates of these claims are included in the ER file. The "final ER claims" file included all the claims identified from both UB92 and HCFA1500 files.

3. Outpatient claims in the UB92 file were identified as those not classified as related to either a hospitalization or ER visit, and constitute claims for services provided in an outpatient setting (e.g. outpatient clinic, home-healthcare use, and physician office). Similarly, outpatient claims in the HCFA1500 file were identified as those not classified as related to either a hospitalization or ER visit. The "final outpatient claims" file included all the claims identified from both UB92 and HCFA1500 files. For each ID, the number of unique dates constituted the total number of outpatient encounters for that ID. An outpatient encounter is defined as any service that generated a CPT code other than 99281-99285, 99288. For outpatient claims, if all the claims for an ID and date were for laboratory or transportation, then these were excluded from the count of outpatient encounters. 
4. ER visits occurring on the same date as that of a hospitalization usually indicate that a decision was made to hospitalize the person based on the ER visit. Hence, all claims from the "final ER claims" file that met this criterion were transferred to the "final hospitalization claims" file.

5. The WV Medicaid medical and prescription files had duplicate claims reflecting the claim adjudication process (such as line items that reflect reversed claims), and were appropriately deleted from the database.

\section{For KY Medicaid:}

1. Hospitalization claims in the UB92 file were identified using the INOUT indicator='IN' (DRG not available in KY files). Also, some claims related to a hospitalization such as those of professional services (e.g. surgeon's fees) will be present in the HCFA1500 file. These claims were identified by using ID and date of admission information, and included in the "final hospitalization claims" file.

2. ER claims in the UB-92 file were identified using the ERIND indicator=' $Y$ '. Similar to hospitalization scenario, all those claims occurring on the same date for an individual for an ER visit in the HCFA1500 file will be included in the final ER claims file. ER visits for which no claim exists in the UB92 file, but claims exist in the HCFA1500 file, were identified by the presence of CPT procedure codes 9928199285, 99288, and all claims occurring on the dates of these claims are included in the ER file. The "final ER claims" file included all the claims identified from both UB92 and HCFA1500 files.

3. ER visits occurring on the same date as that of a hospitalization usually indicate that a decision was made to hospitalize the person based on the ER visit. Hence, all claims from the "final ER claims" file that met this criterion were transferred to the "final hospitalization claims" file.

4. Outpatient claims in the UB92 file were identified as those not classified as related to either a hospitalization or ER visit, and constitute claims for services provided in an outpatient setting (outpatient clinic, home-healthcare use, physician office, etc). Similarly, outpatient claims in the HCFA1500 file were identified as those not classified as related to either a hospitalization or ER visit. The "final outpatient claims" file included all the claims identified from both UB92 and HCFA1500 files. 
For each ID, the number of unique dates constituted the total number of outpatient encounters for that ID. An outpatient encounter is defined as any service that generated a CPT code other than 99281-99285, 99288. For outpatient claims, if all the claims for an ID and date were for laboratory or transportation, then these were excluded from the count of outpatient encounters.

\section{Measurement of Independent Variables}

The multivariate analyses for all phases of the study controlled for several confounding factors, and can be grouped into categories such as demographics, comorbidity, and COPD severity. A description of these covariates follows, and use of these covariates as appropriate is mentioned in the analyses section of each phase.

$\underline{\text { Demographics }}$

1. Age: Calculated as the age at the index date for each phase of the study.

2. Gender: The field 'recipient gender' in the Medicaid eligibility file was used to identify recipient's gender.

3. Race: The field 'recipient race' in the Medicaid eligibility file was used to identify a recipient's race. Race was classified as 'White', 'Black', and 'Other'.

4. County_smokerate: The county_smokerate is a dichotomous variable of the smoking prevalence rate of each recipient's county with values of $0=<31 \%$ and $1=\geq 31 \%$. The cutoff of $31 \%$ was chosen based on the distribution of values in the data. The field 'recipient county' was obtained from the Medicaid eligibility file. County smoking prevalence rates were obtained from statewide-county level Behavioral Risk Factor Surveillance System (BRFSS) data (WVBPH, 2005; KYDPH, 2001; KYDPH, 2002; KYDPH, 2003; KYDPH, 2004). The BRFSS is a telephone health survey sponsored by the Centers for Disease Control and Prevention (CDC) and conducted by each state in the U.S. every year. Current smoking prevalence was obtained in response to the BRFSS question: "Have you smoked at least 100 cigarettes in lifetime and now smoke every day or some days"?

5. State: Data were compiled from two state Medicaid programs: WV and KY for Phase II and III of the study, and the variable denotes the residential state of each recipient. 


\section{Comorbidity}

1. COPD-related comorbid disorders: The presence of seven specific conditions found in higher prevalence in recipients with COPD, and considered to increase healthcare costs for recipients with COPD was determined by presence of a diagnosis on a medical claim (Grasso et al., 1998; Ward et al., 2000). These conditions include upper respiratory tract infections ${ }^{2}$, lower respiratory tract infections ${ }^{3}$, septicemia, allergic rhinitis, other diseases of the lung ${ }^{4}$, congestive heart failure, and disorders of fluid, electrolyte, and acid-base imbalance.

2. Charlson comorbidity index with D'Hoore modification (D'Hoore-CCI): The Charlson index is a validated method of classifying comorbidity to predict short-term and longterm mortality from medical records (Charlson, Pompei, Ales, \& MacKenzie, 1987). The index, as computed, assigns weights (obtained empirically based on relative-risk estimates for death) for 19 major conditions that an individual may have in addition to COPD (see table 7 below). Thus, the index score is the total of assigned weights, and represents a measure of burden of comorbid disease. Therefore, if a person has no chronic conditions then the CCI index score will be 0 . If a person has dementia, ulcer disease, and metastatic solid tumor then the CCI index score will be $1+1+6=8$. Thus, the higher the index score, the more chronic conditions are present. The CCI is computed using the ICD-9 CM codes which include the fourth and fifth digits (Deyo, Cherkin, \& Ciol, 1992). The D'Hoore modification on the other hand, uses the ICD-9 CM codes with only the first three digits for these conditions(D'Hoore, Bouckaert, \& Tilquin, 1996).

The D'Hoore-CCI was computed excluding diagnosis codes for COPD (491, 492, 496) for all analyses. Analyses in which the presence of asthma or CHF was included as a separate variable excluded the codes for asthma and CHF when calculating the D'Hoore CCI.

\footnotetext{
${ }^{2}$ Includes influenza, acute bronchitis and bronchiolitis, bronchitis not specified as acute or chronic, acute nasopharyngitis, acute laryngitis, acute tracheitis, acute laryngotracheitis, acute epiglottis, supraglottis, acute laryngopharyngitis, acute upper respiratory infections of multiple and unspecified sites.

${ }^{3}$ Includes pneumonia from bacterial, viral, other specified organisms, and organism unspecified.

${ }^{4}$ Includes pleurisy, pneumothorax, abscess of lung and mediastinum, pulmonary congestion and hypostasis, pulmonary collapse, interstitial emphysema, compensatory emphysema, pulmonary eosinophilia, acute edema of lung, pulmonary insufficiency following trauma and surgery, allergic bronchopulmonary aspegillosis, and acute and chronic respiratory failure.
} 
TABLE 7: Scoring algorithm for D'Hoore-Charlson comorbidity index

\begin{tabular}{cll}
\hline Weights & \multicolumn{1}{c}{ Conditions } & \multicolumn{1}{c}{ ICD-9 CM codes } \\
\hline 1 & Myocardial infarct & 410,411 \\
& Congestive heart failure & $398,402,428$ \\
& Peripheral vascular disease & $440-447$ \\
& Dementia & $290,291,294$ \\
& Cerebrovascular disease & $430-433,435$ \\
& Chronic Pulmonary disease & $491-493$ \\
& Connective tissue disease & $710,714,725$ \\
& Ulcer disease & 531,534 \\
& Mild liver disease & 571,573 \\
& Hemiplegia & $342,434,436,437$ \\
& Moderate or severe renal disease & $403,404,580-586$ \\
& Diabetes & 250 \\
& Any tumor & $140-195$ \\
& Leukemia & $204-208$ \\
& Lymphoma & $200,202,203$ \\
& Moderate or severe liver disease & $070,570,572$ \\
& Metastatic solid tumor & $196-199$
\end{tabular}

3. Number of other chronic conditions: The number of other chronic conditions not included in the D'Hoore-CCI was also computed to comprehensively assess comorbid burden by the presence of a diagnosis on a medical claim for these conditions. These conditions include hypercholesterolemia, hypertension, other heart disease, AIDS, rheumatoid arthritis, osteoarthritis, gall bladder disease, depression and anxiety, and schizophrenia.

COPD severity in pre-index period

1. Number of canisters of inhaled short-acting beta-agonists (SABA): A prescription claim for an inhaled SABA can be for one or more than one canister. Accordingly, the number of canisters was computed by dividing the quantity dispensed in mg by $\mathrm{mg}$ contained per canister. SABAs included levalbuterol, albuterol, metaproterenol, bitolterol, and terbutaline.

2. Number of canisters of inhaled ipratropium or ipratropium/albuterol: A prescription claim for inhaled ipratropium can be for one or more than one canister. Accordingly, the number of canisters was computed by dividing the quantity dispensed in mg by $\mathrm{mg}$ contained per canister.

3. Number of prescriptions for other short-acting bronchodilators (SABD): The number of prescription claims was computed for other SABD that includes theophylline and oral and powder dosage forms of SABAs.

4. Number of prescriptions for oral corticosteroids (OCS): The number of prescription claims was computed for OCS. OCS includes prednisone, prednisolone, 
methylprednisolone, cortisone, hydrocortisone, dexamethasone, betamethasone, oral triamcinolone, and oral budesonide.

5. Use of nebulized short-acting bronchodilators (SABD): The use of nebulized dosage forms of SABD was categorized as a binary variable ( $0=$ no use; $1=$ use)

6. Use of home oxygen therapy: The use of home oxygen therapy as determined from CPT codes for home oxygen therapy on medical claims was categorized as a binary variable ( $0=$ no use; $1=$ use).

7. Number of Hosp/ER visits for COPD or COPD-related comorbid disorders: The number of hospitalizations and ER visit claims with a primary or secondary diagnosis for a COPD-related comorbid disorder was determined. COPD-related comorbid disorders included asthma, upper respiratory tract infections ${ }^{5}$, lower respiratory tract infections ${ }^{6}$, septicemia, allergic rhinitis, other diseases of the lung $^{7}$, congestive heart failure, and disorders of fluid, electrolyte, and acid-base imbalance.

8. Number of physician visits for COPD or COPD-related comorbid disorders: The number of physician visit claims with a primary or secondary diagnosis for a COPD-related comorbid disorder was determined. COPD-related comorbid disorders included asthma, upper respiratory tract infections ${ }^{8}$, lower respiratory tract infections ${ }^{9}$, septicemia, allergic rhinitis, other diseases of the lung ${ }^{10}$, congestive heart failure, and disorders of fluid, electrolyte, and acid-base imbalance.

9. Presence of asthma: Recipients were classified as having asthma if they had at least one hospitalization, emergency room (ER) visit, or at least two outpatient encounters on different dates in 2002 with a diagnosis code (primary or secondary) for asthma (493.xx).

\footnotetext{
${ }^{5}$ Includes influenza, acute bronchitis and bronchiolitis, bronchitis not specified as acute or chronic, acute nasopharyngitis, acute laryngitis, acute tracheitis, acute laryngotracheitis, acute epiglottis, supraglottis, acute laryngopharyngitis, acute upper respiratory infections of multiple and unspecified sites.

${ }^{6}$ Includes pneumonia from bacterial, viral, other specified organisms, and organism unspecified.

${ }^{7}$ Includes pleurisy, pneumothorax, abscess of lung and mediastinum, pulmonary congestion and hypostasis, pulmonary collapse, interstitial emphysema, compensatory emphysema, pulmonary eosinophilia, acute edema of lung, pulmonary insufficiency following trauma and surgery, allergic bronchopulmonary aspegillosis, and acute and chronic respiratory failure.

${ }^{8}$ Includes influenza, acute bronchitis and bronchiolitis, bronchitis not specified as acute or chronic, acute nasopharyngitis, acute laryngitis, acute tracheitis, acute laryngotracheitis, acute epiglottis, supraglottis, acute laryngopharyngitis, acute upper respiratory infections of multiple and unspecified sites.

${ }^{9}$ Includes pneumonia from bacterial, viral, other specified organisms, and organism unspecified.

${ }^{10}$ Includes pleurisy, pneumothorax, abscess of lung and mediastinum, pulmonary congestion and hypostasis, pulmonary collapse, interstitial emphysema, compensatory emphysema, pulmonary eosinophilia, acute edema
} 
Costs in the pre-index period have been used as a measure of severity in pharmacoecnomic studies using retrospective databases in other disease states. However, the same was not used in the present study for two reasons:

1. In other disease areas, severity measures using retrospective databases are generally restricted to the presence of concomitant conditions/diseases. Prescription drug use is not generally considered as a measure of severity. Hence, these studies include costs that help to serve as a proxy measure of severity. In the case of COPD however, the presence of concomitant conditions/diseases, as well as drugs used in the exacerbated state in COPD serve as an adequate measure of severity in COPD, eliminating the need to include costs in the pre-index period.

2. From an analytical perspective, inclusion of costs in the regression models may introduce violations of assumption, particularly that of multicollinearity and linearity.

\section{Measurement of Outcome Variables}

1. Severe exacerbation: A severe exacerbation was defined as a hospitalization with a primary diagnosis for COPD (ICD-9: 491.xx, 492.xx, 496.xx).

2. Moderate exacerbation: A moderate exacerbation was defined as the presence of an ER visit or a physician visit with a primary diagnosis for COPD (ICD-9: 491.xx, 492.xx, 496.xx) and a prescription for an oral corticosteroid with or without an antibiotic within 3 days following the visit. An exacerbation occurring within 14 days of a previous exacerbation was not counted as a separate exacerbation when computing number of exacerbations (Burge et al., 2003)

3. Total healthcare costs: Total healthcare costs were computed using all the medical and prescription claims of the study sample regardless of diagnoses code. Costs were computed using the amounts reimbursed by Medicaid given on each medical and prescription claim $^{11}$.

of lung, pulmonary insufficiency following trauma and surgery, allergic bronchopulmonary aspegillosis, and acute and chronic respiratory failure.

${ }^{11}$ The hospitalizations claims for WV Medicaid did not include the amount reimbursed by Medicaid, and WV Medicaid reimbursement rates for each DRG was used to calculate hospital costs. For a few DRG codes that did not have a reimbursement value from WV Medicaid, the relative weight of the DRG was used to assign a cost for the DRG. 
4. COPD-related costs: COPD-related costs were computed from the paid amounts of the medical claims with a primary or secondary diagnosis of $\mathrm{COPD}^{11}$, and all the prescription medications used for COPD. COPD-related prescription medications were classified into the following categories: anticholinergics (ipratropium/combination product of ipratropium and albuterol), short-acting beta-agonists (SABA), long-acting beta-agonists (LABA), inhaled corticosteroids (ICS), inhaled corticosteroids/long-acting beta-agonists combination, methylxanthines, oral corticosteroids (OCS), and antibiotics for respiratory infections. COPD-related nursing home costs could not be calculated due to the absence of diagnoses codes on nursing home claims.

5. Death: Death due to any cause except those resulting from an accident, homicide, suicide, or due to injury at work was obtained from the death records from the Bureau of Vital Statistics. 


\section{Analyses of Cost Data}

The following paragraph details the analytical approach used for multivariate analyses when the dependent variable was the cost incurred by sample recipients. For cost data where $0 \%$ or less than $2 \%$ of the sample incurred costs (e.g. total healthcare costs or outpatient costs), only one regression model was estimated. Since cost data were positively skewed, costs were log-transformed, and consequently semi-logarithmic ordinary least squares (OLS) regression was used (Manning WG \& Mullahy J, 2001). Adjusted cost estimates were computed by retransformation of the log-transformed costs using Duan's smearing correction factor (Duan N, 1983). The presence of heteroskedasticity in the regression models was determined using the White's test or the Breusch-Pagan/Cook-Weisburg test and also by examining the graphical distribution of the residuals. If the semi-logarithmic OLS model demonstrated heteroskedasticity, precluding retransformation without introducing bias (Manning WG, 1998), a generalized linear model (GLM) using a gamma distribution with a $\log$ link was used. The latter have the advantage of estimating the adjusted costs directly without the need for retransformation while simultaneously using log-transformed costs in its estimation, provided the log-scale error distribution is not kurtotic (Manning WG et al., 2001). In a few instances where the GLM model was not found to be a good fit, the heteroskedastic semi-log OLS model was used, since a bias would only be reflected in the retransformed costs and not the impact of the primary independent variable. In all of these instances, the heteroskedasticity was mild as depicted in a graph of the residuals versus the fitted values.

Unlike total healthcare costs, where all recipients had some costs (because all recipients are identified by presence of a medical claim), individual cost components like hospitalization costs are not present for all recipients. Thus, these data have two primary characteristics: a significant proportion with zero costs, and a positively skewed distribution for those with costs. Therefore, a two-part model was used with a logistic model for the first part, and a semi-log OLS model or a GLM model for the second part (Duan N, Manning WG, Morris CN, \& Newhouse JP, 1983). Adjusted costs estimated from two-part models were computed by multiplying the adjusted probability obtained from the logistic regression model (part 1) with the retransformed costs estimated from the semi-log OLS or the predicted cost from the GLM model (part 2) (Duan N et al., 1983). 
The beta coefficients of all semi-log OLS and GLM models were interpreted using a correction by Halverson and Palmquist with a modification by Kennedy (Halvorsen R \& Palmquist R, 1980; Kennedy P, 1981). Outliers were excluded as considered appropriate using cutoff values for residuals and Cook's distance. All regression models were tested for multicollinearity using cutoff values of the variance inflation factor for each variable. None of the models had multicollinearity issues. All statistical analyses were evaluated at $\alpha=0.05$. 


\section{DESIGN AND ANALYSIS OF STUDY GOALS AND OBJECTIVES}

\section{Phase I}

Study design: A cross-sectional design was used for both objectives in phase I using data for calendar year 2002 (most recent annual data available).

\section{Research Objective 1: To estimate mean incremental costs incurred by recipients with COPD enrolled in West Virginia and Kentucky Medicaid.}

Study sample: Medicaid recipients having at least one hospitalization, emergency room (ER) visit, or at least two outpatient encounters on different dates with a primary diagnosis code for COPD (ICD-9: 491.xx, 492.xx, and 496.xx) in 2002 were identified as having COPD. Prescription claims were not used for purposes of identifying the COPD population because of the possibility of diagnostic misclassification with patients with bronchitis and asthma. Only recipients between 35-64 years of age and those continuously eligible during 2002 were included. Recipients without COPD were identified based on the absence of a claim with an ICD-9 CM code for COPD in any diagnosis field. For each recipient with COPD, one nonCOPD recipient was matched on age and gender.

Outcome measure: Based on the type of medical care received, total healthcare costs were classified into five main components: hospital, ER, outpatient, prescription, and nursing home. Physician visit costs were also computed as a sub-component of outpatient costs.

Analyses: A description of the study sample by COPD status in terms of their demographic and comorbidity characteristics was provided using means and proportions as considered appropriate. Proportions of recipients with at least one hospitalization visit, ER visit, outpatient encounter, physician visit, and any use of nursing home were reported by COPD status. The number and cost of hospitalization visits, ER visits, outpatient encounters, physician visits, and nursing home (number of nursing home visits per recipient could not be

calculated from data source) per recipient was compared between recipients with and without COPD. The mean total healthcare cost incurred by recipients with COPD was calculated as the difference in mean total healthcare costs between recipients with and without COPD. 
COPD-related utilization and costs in relation to total healthcare costs for recipients with COPD was also analyzed. McNemar test was used to compare proportions of recipients with and without COPD using different healthcare cost components, and the Wilcoxon signed rank test was used for the number and cost per recipient of cost components. Multivariate analyses were also conducted controlling for race, county_smokerate, D'Hoore-CCI, and number of other chronic conditions to determine differences in total healthcare costs as well as individual cost components between recipients with and without COPD.

\section{Research Objective 2: To compare mean COPD-related costs among recipients with COPD with and without concomitant asthma enrolled in West Virginia and Kentucky Medicaid.} Study sample: Recipients with COPD identified in objective 1 were classified as having asthma if they had at least one hospitalization, emergency room (ER) visit, or at least two outpatient encounters on different dates in 2002 with a diagnosis code (primary or secondary) for asthma (493.xx).

Outcome measure: COPD-related total costs as well as for each component (hospital, ER, outpatient, and prescription) were computed for recipients with COPD with and without a concomitant diagnosis of asthma from the paid amounts of the medical claims with a primary or secondary diagnosis of COPD, and all the prescription medications used for COPD. COPD-related nursing home costs could not be calculated due to the absence of diagnoses codes on nursing home claims.

Analyses: A description of the study sample by asthma status in terms of their demographic and comorbidity characteristics was provided using means and proportions as considered appropriate. Proportions of recipients with at least one COPD-related hospitalization visit, ER visit, outpatient encounter, physician visit, and any use of nursing home were reported by concomitant asthma status. The number and cost of COPD-related hospitalization visits, ER visits, outpatient encounters, and physician visits per recipient was compared between recipients with COPD with and without concomitant asthma (number of nursing home visits per recipient could not be calculated from data source). The incremental cost per recipient due to asthma for recipients with COPD was calculated as the difference in total COPD- 
related costs per recipient between recipients with COPD with and without concomitant asthma. Chi-square tests were used to compare proportions of recipients with and without asthma using different healthcare cost components, and the Mann-Whitney U test or t-test (as appropriate for the distribution) was used for the number and cost per recipient of cost components. Multivariate analyses were also conducted controlling for age, gender, race, county_smokerate, presence of upper respiratory tract infections, lower respiratory tract infections, septicemia, allergic rhinitis, other diseases of the lung, congestive heart failure, and disorders of fluid, electrolyte, and acid-base imbalance, D'Hoore-CCI excluding asthma and $\mathrm{CHF}$, and number of other chronic conditions to determine differences in total healthcare costs as well as individual cost components between recipients with and without COPD.

\section{Phase II}

Phase II of the study compares costs and exacerbations of recipients initiating drug therapy. Part 1 of phase II compares these outcomes between recipients initiating maintenance therapy with ipratropium (i.e. use of combination product of ipratropium and albuterol, IPR/ALB) or monotherapy with inhaled long-acting beta-agonist (LABA).

Part 1

Study sample: The study sample for both objectives in part 1 of Phase II was identified as shown in figure 6. Sample recipients were identified as those having a prescription claim for the combination product of ipratropium and albuterol (IPR/ALB) or inhaled long-acting betaagonist (LABA) between WV: July 1, 1998 to June 30, 2002 and KY: January 1, 1999 and December 31, 2002. An index prescription was defined as the first chronologically occurring prescription for which no other claims for these index drugs are found in the 12 months preceding the date of the index prescription. This procedure identified an inception cohort of new users of medication to indicate the start of drug therapy. The date of the index prescription was termed as the index date, and the 12 months before the index date was designated as the pre-index period. The follow-up period for outcome assessment was defined as the 12 months after the index date. The study period spanned 24 months for each recipient. An intent-to-treat approach, similar to that used in randomized controlled trials was used for the analyses. Thus, recipients with COPD identified to be in a drug therapy 
group were considered as using that therapy during the entire follow-up periiod regardless of therapy discontinuations that may occur beyond the index date.

FIGURE 6: Identification of study sample for Part 1 of Phase II

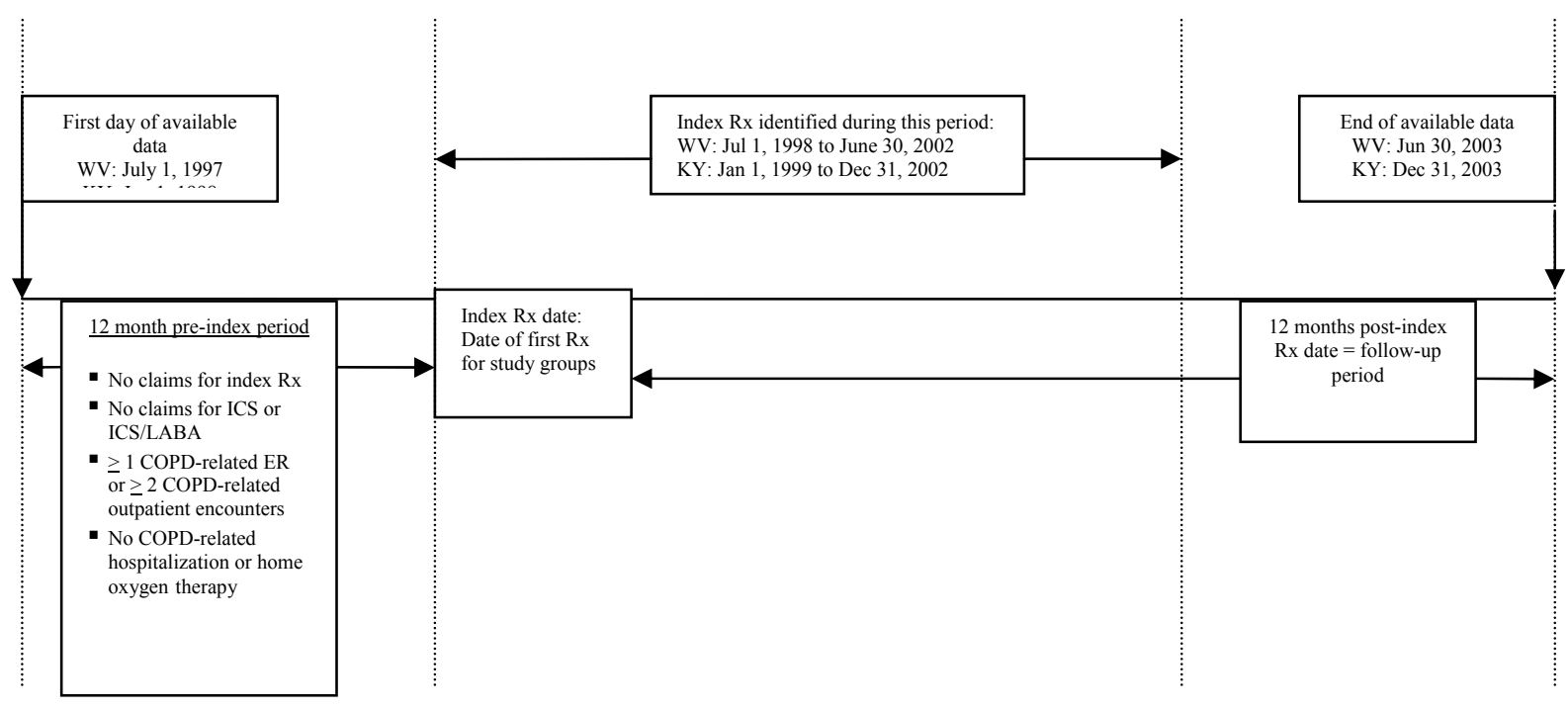

Drug therapy groups:

- IPR/ALB: Recipients who fill a prescription for the combination product of ipratropium/albuterol, and no claims for inhaled long-acting beta-agonists in the 30-day post-index period. Additionally, recipients are required to have one more prescription claim for IPR/ALB in the follow-up period.

- LABA: Recipients with a prescription claim for inhaled long-acting beta-agonist and no prescription claims for ipratropium or for the combination product of ipratropium/albuterol in the post-index 30-day period. Additionally, recipients are required to have one more prescription claim for LABA in the follow-up period.

- Both drug therapy groups could have prescriptions claims for short-acting beta-agonists, methylxanthines, and oral corticosteroids.

\section{Inclusion criteria:}

- Recipients with at least one ER visit or at least two outpatient encounters on different dates in the pre-index period with a primary or secondary diagnosis of COPD (ICD-9: 491.xx, 492.xx, 496.xx).

- Recipients at least 35 years of age at the index date. 
- Recipients continuously eligible one year before and one year after the index date. Exclusion criteria:

- Recipients with claims for a hospitalization with a primary or secondary diagnosis of COPD (ICD-9: 491.xx, 492.xx, 496.xx) in the pre-index period.

- Recipients with claims for home oxygen therapy in the pre-index period.

- Recipients with claims for an inhaled corticosteroid (ICS) or a combination product of an inhaled corticosteroid and a long-acting beta-agonist (ICS/LABA) in the pre-index period, or in the 30 days following the index date.

- Recipients with a diagnosis of respiratory cancer, cystic fibrosis, fibrosis due to tuberculosis, and bronchiectasis during the study period were excluded to avoid misdiagnosis.

- Recipients over 64 years of age and those with Medicare eligibility were excluded because of insufficient capture of claims.

- Managed care recipients were excluded as they do not have all their utilization information in the claims database.

\section{Research Objective 3: To compare the risk and frequency of exacerbations between recipients with COPD who initiated maintenance therapy with ipratropium or monotherapy with inhaled long-acting beta-agonist.}

Study design and outcome measure: A historical cohort design was used for objective 3 . The outcome was an exacerbation (moderate or severe) for COPD. Differences between groups in risk of an exacerbation following the index date were determined as shown in figure 7. Starting with the index date, recipients were followed until an exacerbation occurred or till the end of the 12-month follow-up period, whichever occurred first. 
FIGURE 7: Historical cohort design to determine differences in time to first exacerbation (Phase II - Part 1)

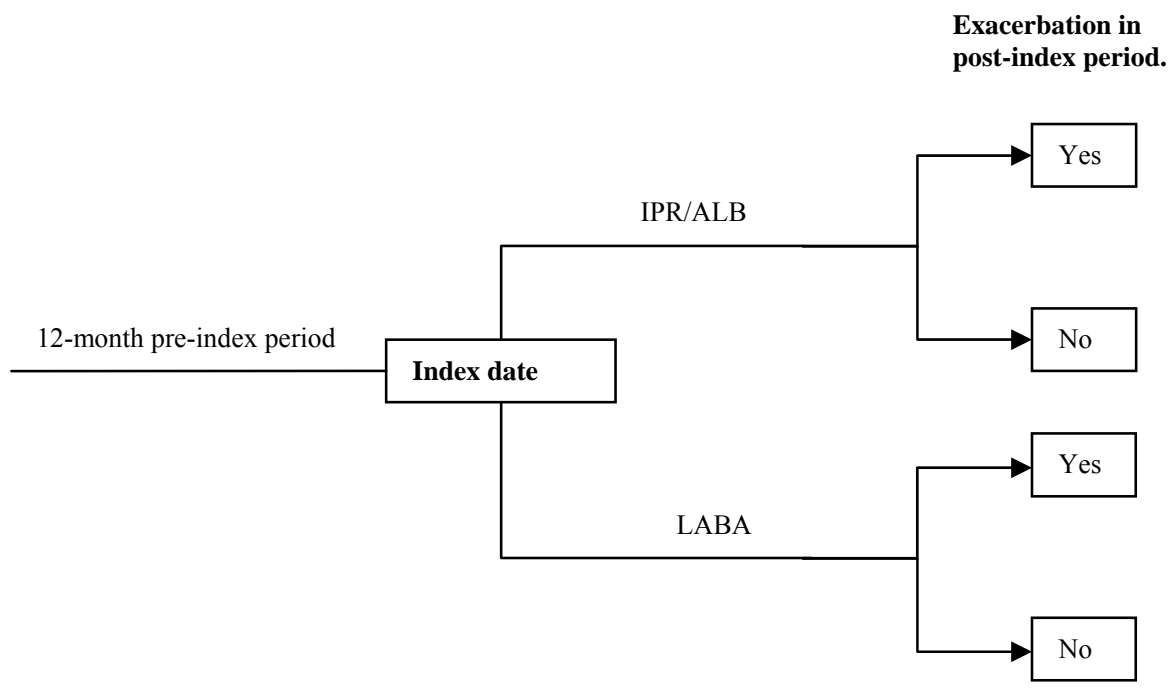

A difference in frequency of exacerbations following the index date was determined as shown in figure 8 . Starting with the index date, each recipient was followed for a total of 12 months and the total number of exacerbations occurring in this period was calculated.

FIGURE 8: Historical cohort design to determine differences in number of exacerbations (Phase II - Part 1)

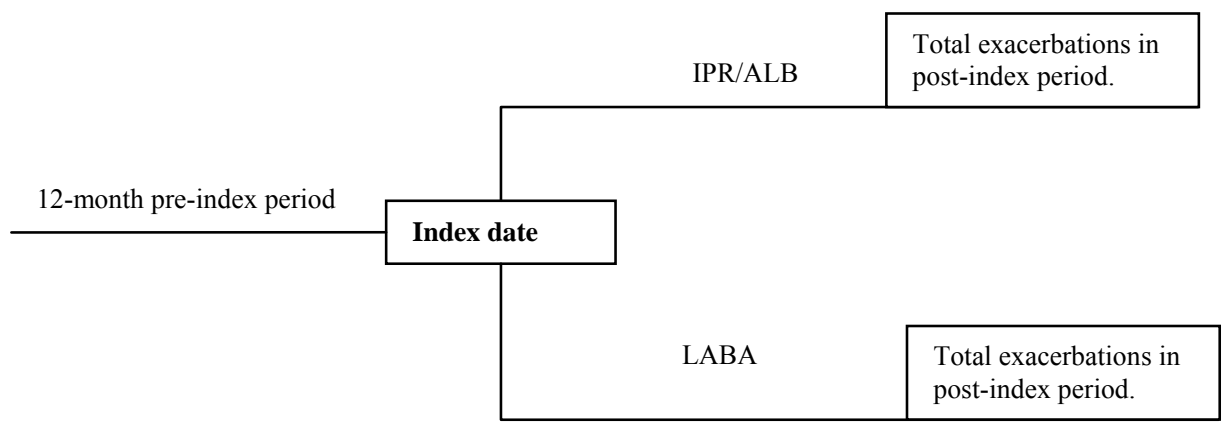


Research Objective 4: To compare COPD-related costs between recipients with COPD who initiated maintenance therapy with ipratropium or monotherapy with inhaled long-acting beta-agonist.

Study design and outcome measure: A historical cohort design was used for objective 4 as shown in figure 9. COPD-related costs accruing in the post-index period were computed for each drug therapy group.

FIGURE 9: Historical cohort design to determine differences in COPD-related costs (Phase II Part 1)

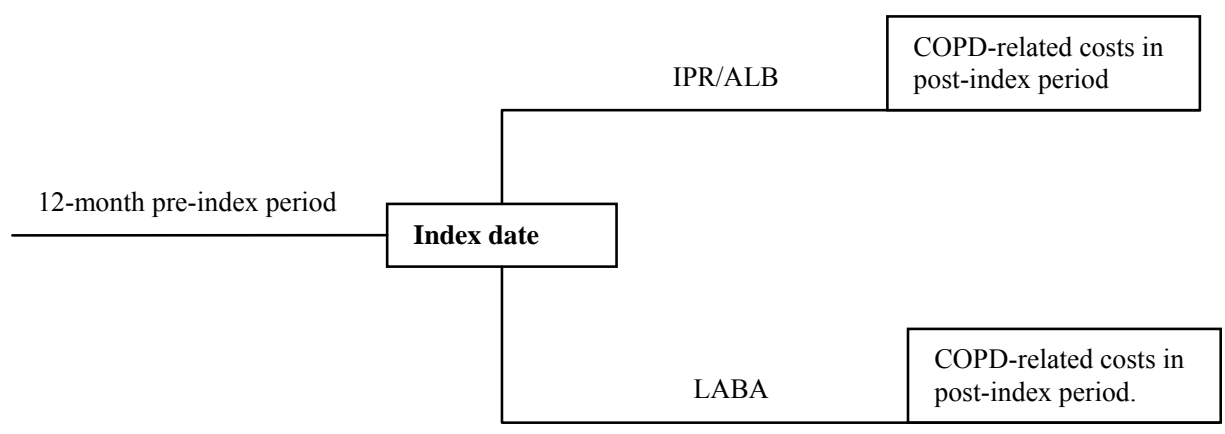

Analyses for research objective $3 \& 4$ :

Description of the study sample at baseline was done using descriptive statistics. Univariate differences in proportion experiencing an exacerbation were analyzed using chisquare tests, and time to exacerbation was analyzed using the log-rank test. Differences in total COPD-related costs and individual cost components were determined using MannWhitney tests. Multivariate analyses controlled for covariates in the pre-index period including demographic characteristics (age, gender, race, state, county smoking prevalence rate), overall comorbidity (D'Hoore CCI, number of other chronic conditions), and measures of COPD severity (number of canisters of inhaled short-acting beta-agonists (SABA), number of prescriptions of other short-acting bronchodilators (SABD), number of prescriptions of oral corticosteroids (OCS), use of nebulized prescriptions of SABD, number of hospitalizations/ER visits for COPD or COPD-related comorbid disorders, number of physician visits for COPD or COPD-related comorbid disorders, and presence of asthma). Survival analysis techniques were used to determine differences in time to an exacerbation. The proportional hazards assumption was tested using the global test of proportional hazards, and the assumption was satisfied. Consequently, a Cox-proportional hazards regression was 
used to determine differences in time to an exacerbation controlling for the above-mentioned covariates. A logistic regression was used to determine differences in the probability of experiencing an exacerbation controlling for the above-mentioned covariates.

The frequency of exacerbations represents count level data i.e. a non-negative integervalued random variable, and accordingly differences in the frequency of exacerbations can be determined using the Poisson or the negative binomial regression model (depending on the relationship between the variance and the mean) (Long JS, 1997). However, 84\% of our sample have no exacerbations (i.e. number of exacerbations $=0$ ). In such a situation, a zeroinflated Poisson or zero-inflated negative binomial regression model is appropriate (Greene WH, 1994). The zero-inflated models allow overdispersion by changing the mean structure to explicitly model the production of zero counts. The suitability of a zero-inflated model was tested by the Vuong statistic. If it is positively significant, then it favors the zeroinflated models, while those that are significantly negative favor the original Poisson or negative binomial regression model. Our model showed a significantly positive Vuong's statistic, and accordingly a zero-inflated model was used. The choice between Poisson and negative binomial model was determined by the likelihood ratio test of $\alpha=0$. Since the value of $\alpha$ was found to be 0 , and the $p$-value $=1.00$, the zero-inflated Poisson model was used to determine differences in frequency of exacerbations. Multivariate differences in COPDrelated costs were analyzed using the approach mentioned for analyses of cost data. 


\section{Part 2}

The second part of phase II compares outcomes of exacerbations and costs between recipients initiating combination therapy with an inhaled corticosteroid and long-acting betaagonist (ICS/LABA), inhaled corticosteroid monotherapy (ICS alone), long-acting betaagonist monotherapy (LABA alone), and those on short-acting bronchodilators (SABD). Study sample: The study sample for objectives 5, 6 and 7 in part 2 of phase II, was identified as shown in figure 10. Sample recipients were identified as those having a prescription claim for a combination product of inhaled corticosteroid and long-acting beta-agonist (ICS/LABA), inhaled corticosteroid (ICS), inhaled long-acting beta-agonist (LABA), or short-acting bronchodilators (SABD) including anticholinergics (IPR or IPR/ALB), shortacting beta-agonists, and methylxanthines between WV: July 1, 1998 to June 30, 2002 and KY: January 1, 1999 and December 31, 2002. An index prescription was defined as the first chronologically occurring prescription for which no other claims for ICS/LABA, ICS, or LABA are found in the 12 months preceding the date of the index prescription. This procedure identified an inception cohort of new users of medication to indicate the start of drug therapy. The date of the index prescription was termed as the index date, and the 12 months before the index date was designated as the pre-index period. The follow-up period for outcome assessment was defined as the 12 months after the index date. The study period spanned 24 months for each recipient. An intent-to-treat approach was used for the analyses. Thus, recipients with COPD identified to be in a drug therapy group were considered to be using that therapy during the entire follow-up period regardless of therapy discontinuations that may occur beyond the index date.

FIGURE 10: Identification of study sample for Part 2 of Phase II

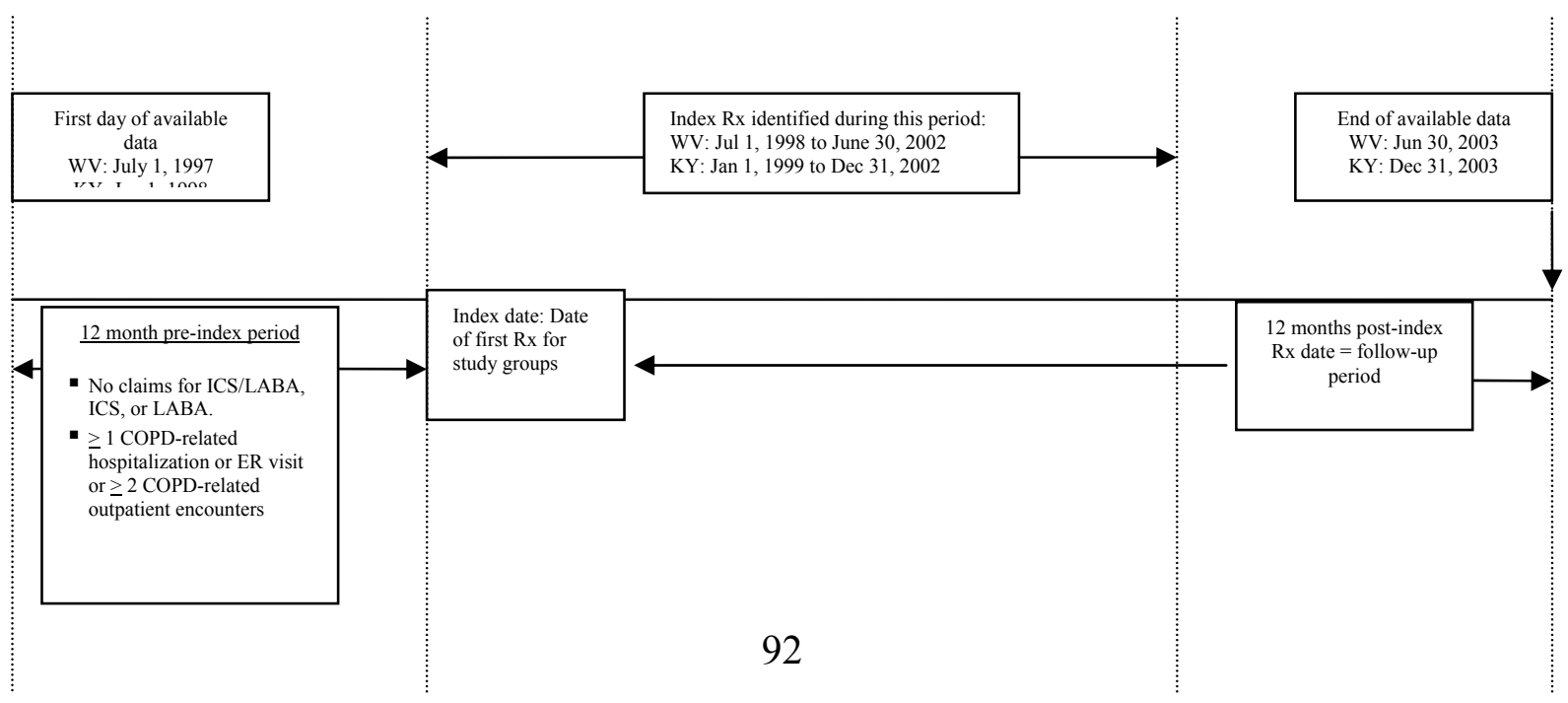




\section{Drug therapy groups:}

- ICS/LABA: Recipients who filled a prescription for the combination product of an ICS/LABA. Additionally, recipients are required to have one more prescription claim for combination product of ICS/LABA in the follow-up period.

- ICS: Recipients who filled a prescription for an ICS and no prescription for LABA or combination product of ICS/LABA through the end of the follow-up period.

Additionally, recipients are required to have one more prescription claim for ICS in the follow-up period.

- LABA: Recipients who filled a prescription for LABA and no prescription for an ICS or combination product of ICS/LABA through the end of the follow-up period. Additionally, recipients are required to have one more prescription claim for LABA in the follow-up period.

- SABD: Recipients who filled a prescription for anticholinergics (IPR or IPR/ALB), shortacting beta-agonists, or methylxanthines but did not fill a prescription for ICS, LABA, or combination product of ICS/LABA through the end of the follow-up period. The SABD group was required to have at least two prescriptions of short-acting bronchodilators in the pre-index period of which at least one was for ipratropium. This was done to avoid the possibility of including recipients with mild COPD who use SABD on an as-needed basis. Additionally, recipients are required to have one more prescription claim for $\mathrm{SABD}$ in the follow-up period.

- All therapy groups could also have prescriptions for short-acting bronchodilators.

\section{Inclusion criteria:}

- Recipients with at least one hospitalization or ER visit or at least two outpatient encounters on different dates in the pre-index period with a primary or secondary diagnosis of COPD (ICD-9: 491.xx, 492.xx, 496.xx).

- Recipients at least 35 years of age at the index date.

- Recipients continuously eligible one year before and one year after the index date. Exclusion criteria:

- Recipients with prescription claims for ICS, LABA, or ICS/LABA combination product in the pre-index period. 
- Recipients with a diagnosis of respiratory cancer, cystic fibrosis, fibrosis due to tuberculosis, and bronchiectasis during the study period were excluded to avoid misdiagnosis.

- Recipients over 64 years of age and those with Medicare eligibility were excluded because of insufficient capture of claims.

- Managed care recipients were excluded as managed care recipients do not have all their utilization information in the claims database.

Research Objective 5: To compare the risk of a severe exacerbation among recipients with COPD who initiate therapy with a combination of an inhaled corticosteroid and a longacting beta-agonist, inhaled corticosteroid alone, inhaled long-acting beta-agonist alone, or only short-acting bronchodilators.

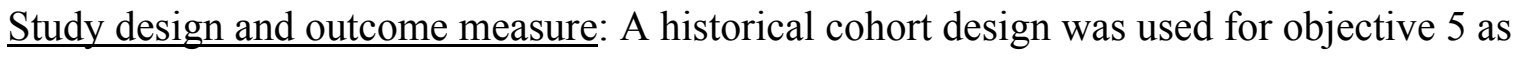
shown in figure 11. The outcome was a severe exacerbation of COPD. Recipients were followed for a period of 12 months until a severe exacerbation occurred or till the end of 12month follow-up period, whichever occurred first. Exacerbations were counted 14 days after the index date to avoid protopathic bias i.e. the bias that results when the exposure occurs as a direct result of the outcome. Thus, if an exacerbation was the reason that an ICS was prescribed, this might inadvertently increase the number of exacerbations for the group being prescribed a drug with an inhaled corticosteroid component. 
FIGURE 11: Historical cohort design to determine differences in time to first severe exacerbation (Phase II - Part 2)

Severe exacerbation in post-index period.

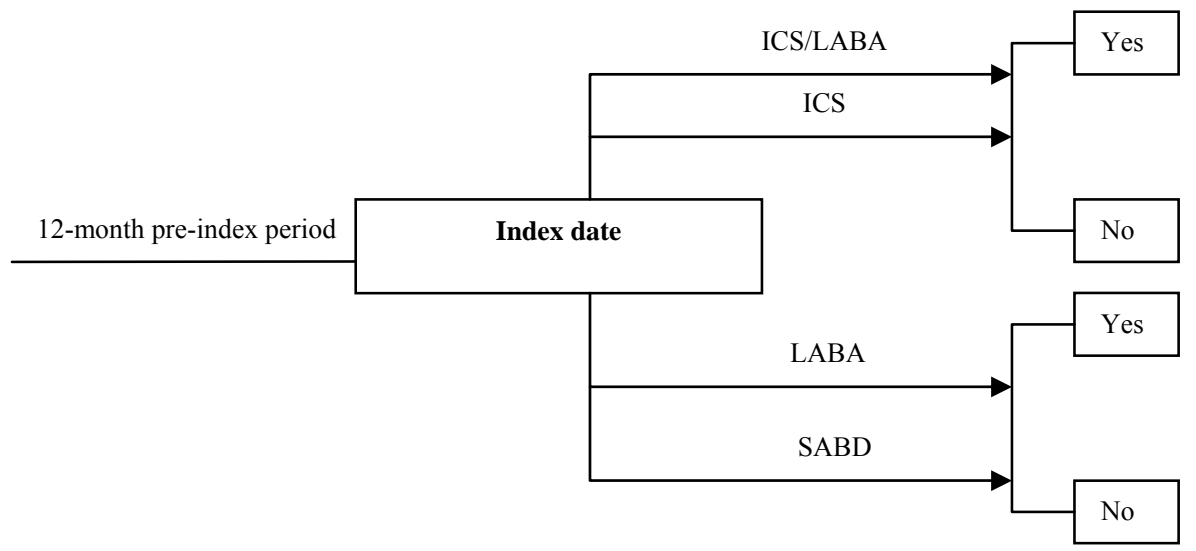

Research Objective 6: To compare the frequency of moderate exacerbations among recipients with COPD who initiate therapy with a combination of an inhaled corticosteroid and a long-acting beta-agonist, inhaled corticosteroid alone, inhaled long-acting betaagonist alone, or only short-acting bronchodilators.

Study design and outcome measure: A historical cohort design was used for objective 6 . The outcome was a moderate exacerbation of COPD. Each recipient was followed for a period of 12 months after the index date and the total number of moderate exacerbations occurring in this period was calculated for each individual group as shown in figure 12. Exacerbations were counted 14 days after the index date to avoid protopathic bias i.e. the bias that results when the exposure occurs as a direct result of the outcome. Thus, if an exacerbation was the reason that an ICS was prescribed, this might inadvertently increase the number of exacerbations for the group being prescribed a drug with an inhaled corticosteroid component. 
FIGURE 12: Historical cohort design to determine differences in number of moderate exacerbations (Phase II - Part 2)

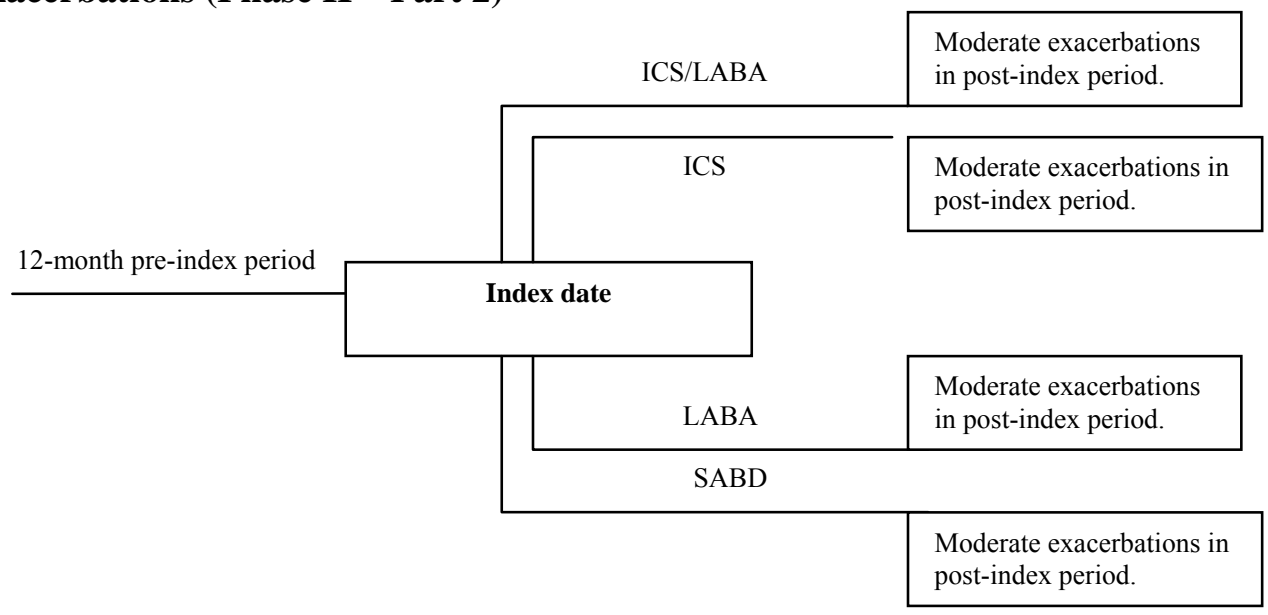

Research Objective 7: To compare COPD-related costs among recipients with COPD who initiate therapy with a combination of an inhaled corticosteroid and a long-acting betaagonist, inhaled corticosteroid alone, inhaled long-acting beta-agonist alone, or only short-acting bronchodilators.

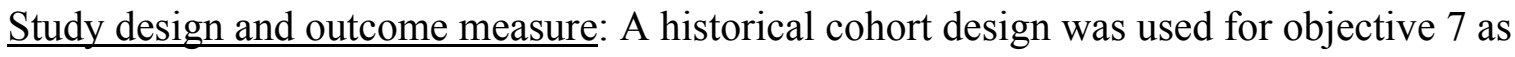
shown in figure 13. COPD-related costs accruing in the 12 months follow-up period were computed for each drug therapy group.

FIGURE 13: Historical cohort design to determine differences in COPD-related costs (Phase II Part 2)

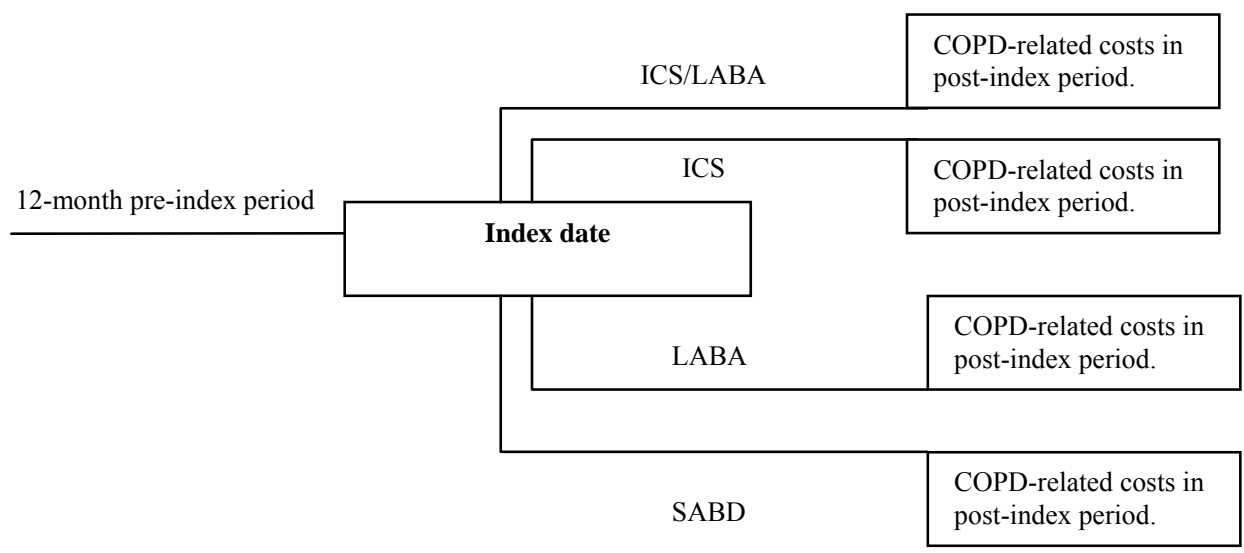


Analyses for research objective 5,6, and 7:

Four drug therapy cohorts were formed for part 2 of Phase II. Three primary comparisons were of interest:

1. ICS/LABA vs. SABD

2. ICS monotherapy vs. SABD

3. ICS/LABA vs. LABA

The first two comparisons assess whether therapy with ICS improves outcomes relative to therapy with SABD. The third comparison assesses if addition of ICS to LABA monotherapy improves outcomes.

Since the study is observational in nature, there is an inherent selection bias in the creation of these drug therapy cohorts. Specifically, all three comparisons share a common aspect with regard to the comparator groups: one group has been given an ICS. The treatment algorithm for COPD reserves therapy with inhaled corticosteroids for those with severe COPD. Hence, by definition there exist differences on observed characteristics between the groups that might lead to biased estimates of the effect of treatment. Several techniques exist to account for the impact of selection bias on treatment effects. The use of propensity scores is one such technique that was used to account for selection bias when analyzing outcomes for part 2 of Phase II (D'Agostino, Jr., 1998).

The propensity score for an individual is defined as the probability of being treated with an ICS conditional on (or based only on) the individual's covariate values. Thus, if two persons, one found to be in the ICS group and the other in the non-ICS group, had the same propensity score, then these two persons would have the same predicted probability of being assigned to the ICS group (Rubin, 1997). Thus, it a measure of the likelihood that a person would have been treated using only their covariate scores. It is obtained from a logistic regression that predicts the probability of being in the ICS therapy group based on several factors $^{12}$. The propensity score so obtained provides a scalar summary of the covariate

\footnotetext{
${ }^{12}$ Factors considered to predict the probability of being treated with an ICS included demographic characteristics (age, gender, race, state, county smoking prevalence rate), overall comorbidity (D'Hoore CCI, number of other chronic conditions), and measures of COPD severity (number of canisters of inhaled shortacting beta-agonists (SABA), number of prescriptions of other short-acting bronchodilators (SABD), number of prescriptions of oral corticosteroids (OCS), use of nebulized prescriptions of SABD, use of hospitalizations/ER visits for COPD or COPD-related comorbid disorders, number of physician visits for COPD or COPD-related comorbid disorders, presence of asthma, and use of home oxygen therapy).
} 
information, and represents the probability of being treated. Using this probability to adjust the estimated treatment effect has the advantage of creating a 'quasi-randomized' experiment. In observational studies, the propensity scores are used primarily to reduce bias and increase precision, using three common techniques: matching, stratification, and regression adjustment (D'Agostino, Jr., 1998).

For the two comparisons in part 2 of Phase II, matched samples were created using the technique of nearest available matching on the estimated propensity score (Parsons L.S., 2000). This matching method consists of randomly ordering the treated and control subjects, then selecting the first treated subject and finding the control subject with the closest propensity score. Both subjects are then removed from consideration for matching and the next treated subject is selected. For both comparisons, The ICS comparator groups had a larger number of people compared to those without ICS. Thus, each recipient in the non-ICS groups (control) were matched to recipients from the ICS group (treated) based on the propensity score using the nearest neighbor matching technique.

Before matching, baseline characteristics were compared between therapy groups using ttests or Mann-Whitney tests for continuous variables and chi-square tests for categorical variables. After matching, baseline characteristics were compared between therapy groups using Wilcoxon signed rank tests for continuous and McNemar's test for categorical variables. The ability of propensity scores to reduce bias was assessed by assessing the balance in covariates after matching. Since a balance was found for all covariates, univariate statistics was used to compare therapy groups on outcomes of exacerbations and costs.

Research objective 5 compares the risk of a severe exacerbation between therapy groups, and McNemar's test was used to assess statistical significance and obtain the odds ratio estimate. Research objective 6 compares the frequency of moderate exacerbations between therapy groups, and the Wilcoxon signed rank test was used to determine differences in number of moderate exacerbations in the follow-up period. Similarly, for comparison of COPD-related costs in research objective 7, the Wilcoxon signed rank test was used. 


\section{Phase III}

Phase III assesses the impact of ICS on mortality using two different study designs. Objective 8 uses a case-control and Objective 9 uses a historical cohort study design to ascertain this association. Objective 9 also attempts to test the hypothesis that any impact of ICS on mortality is mediated by the impact of ICS on severe exacerbations.

\section{Research Objective 8: To compare the odds of exposure to inhaled corticosteroids between those who died and those who did not die during the study period (case-control study design).}

Study sample: The study sample for objective 8 of phase III was identified as shown in figure 14. An initial subset of recipients between 35-64 years of age having at least one medical claim with a primary diagnosis code for COPD (ICD-9: 491.xx, 492.xx, and 496.xx) between the period WV: July 1, 1997 to June 30, 2003 and KY: January 1, 1998 to December 31, 2003 were identified. Unique identification numbers of the recipients from this initial subset were then linked to death records from the Bureau of Vital Statistics to determine recipients who died between WV: July 1, 1998 to June 30, 2003 and KY: January 1, 1999 to December $31,2003$.

Recipients who died were designated as cases, and all other recipients were eligible to be selected as controls, which are the comparators in a case-control study design. Inclusion and exclusion criteria were applied to determine the number of cases and controls meeting study eligibility criteria. Eligible controls were selected and matched to each case by age, gender, and ethnicity to obtain the final study sample for objective 8 . The index date is the date of death for a case. The index date for each control is the date of death of its matched case. The 12 month period prior to the index date is the pre-index period, and also represents the study period for each recipient. 
FIGURE 14: Identification of study sample for research objective 8 of Phase III

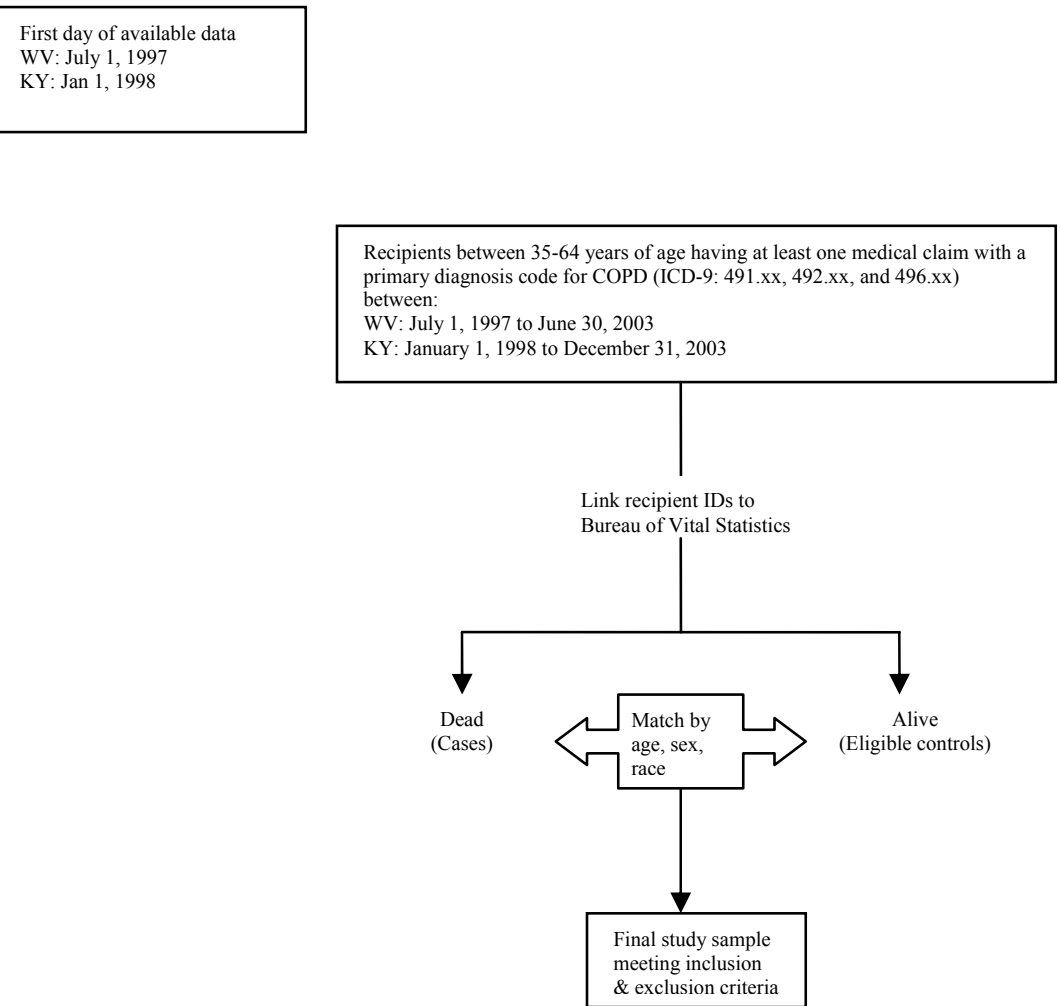

Inclusion criteria:

- Recipients with at least one hospitalization, emergency room (ER) visit, or at least two outpatient encounters on different dates with a primary diagnosis code for COPD (ICD-9: 491.xx, 492.xx, and 496.xx) were identified as having COPD.

- Recipients at least 35 years of age at the first day of available data.

- Recipients continuously eligible for one year before the index date.

\section{Exclusion criteria:}

- Recipients with a diagnosis of respiratory cancer, cystic fibrosis, fibrosis due to tuberculosis, and bronchiectasis during the study period were excluded.

- Recipients over 64 years of age and those with Medicare eligibility were excluded because of insufficient capture of claims.

- Managed care recipients were excluded as managed care recipients do not have all their utilization information in the claims database.

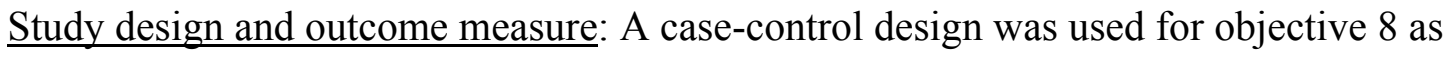
demonstrated in figure 15. In accordance with the case-control design, prescription claims in 
the 12 months prior to the index date were examined for cases and controls. Recipients with at least two prescription claims for ICS in this retrospective period were classified as ICS users, and those without any claims were classified as non-ICS users. Recipients with only one prescription claim for ICS in the pre-index period were excluded to avoid the possibility of misclassification.

FIGURE 15: Case-control design to determine impact of ICS on mortality

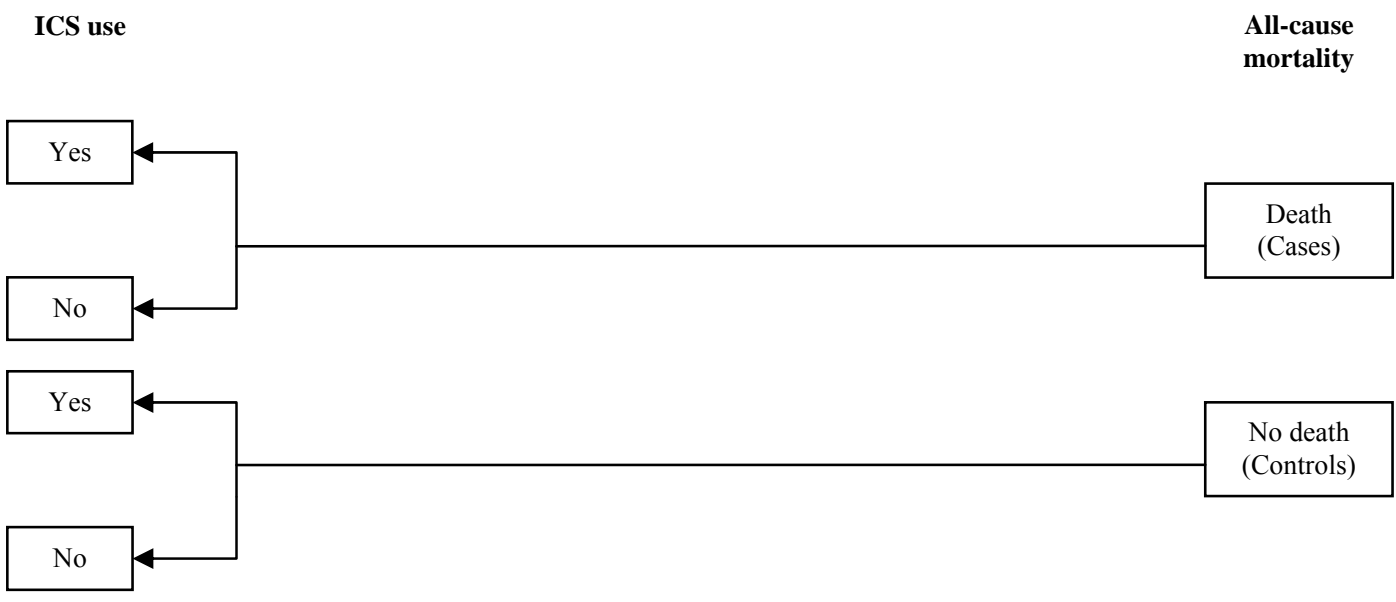

Analyses for Research Objective 8:

Description of the study sample in the pre-index period was done using descriptive statistics. McNemar test was used to compare cases and controls on categorical variables, and the Wilcoxon signed rank test was used for continuous variables. A conditional logistic regression was used controlling for covariates in the pre-index period including county smoking prevalence rate, overall comorbidity (D'Hoore CCI, number of other chronic conditions), and measures of COPD severity (number of canisters of inhaled short-acting beta-agonists (SABA), number of canisters of inhaled ipratropium (IPR), number of prescriptions of other doseform short-acting bronchodilators (SABD), number of prescriptions of oral corticosteroids (OCS), use of nebulized prescriptions of SABD, number of hospitalizations/ER visits for COPD or COPD-related comorbid disorders, number of physician visits for COPD or COPD-related comorbid disorders, presence of asthma, and use of home oxygen therapy). 


\section{Research Objective 9: To conduct a mediation analysis to examine the relationship}

between use of ICS, severe exacerbations, and death (historical cohort study design).

Study sample: The study sample for objective 9 of phase III was identified as shown in figure 16. Sample recipients were identified as those having a prescription claim for an inhaled corticosteroid (defined as a prescription claim for inhaled corticosteroid or for the combination product of an inhaled corticosteroid and a long-acting beta-agonist), or use of other COPD-related drugs (defined as a prescription claim for long-acting beta-agonists, anticholinergics (ipratropium or combination product of ipratropium and albuterol), shortacting beta-agonists, or methylxanthines). An index prescription was defined as the first chronologically occurring prescription for which no other claims for these index drugs are found in the 12 months preceding the date of the index prescription. This procedure identified an inception cohort of new users of medication to indicate the start of drug therapy. The date of the index prescription was termed as the index date, and the 12 months before the index date was designated as the pre-index period. The follow-up period was defined as 12 months after index date. The study period spanned 24 months for each recipient. An intentto-treat approach was used for the analyses. Thus, recipients with COPD identified to be in a drug therapy group were considered to be using that therapy during the entire follow-up period regardless of therapy discontinuations that may occur in the follow-up period.

FIGURE 16: Identification of study sample for research objective 9 of Phase III

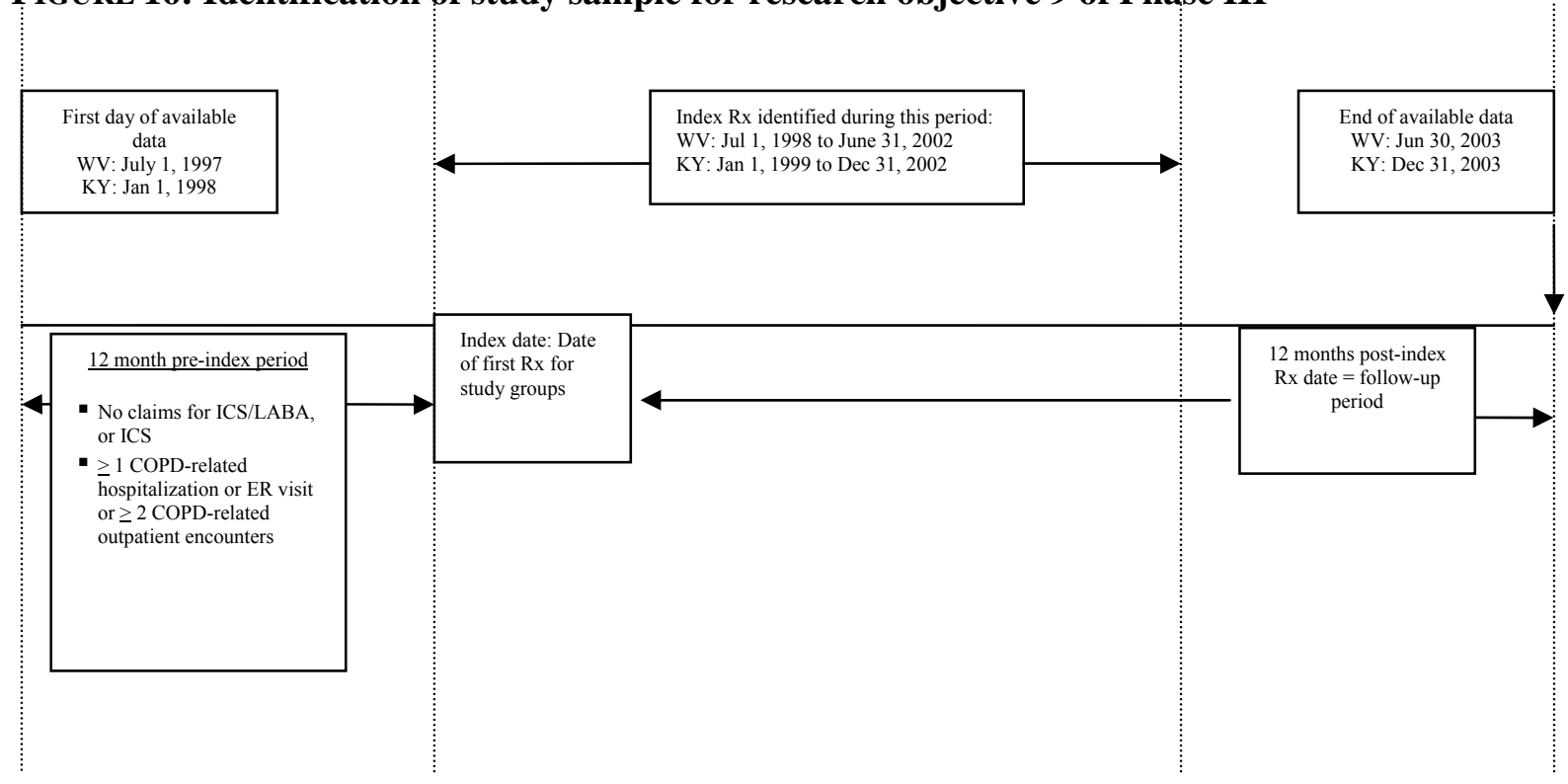




\section{Drug therapy groups:}

- ICS: Recipients who filled a prescription for an ICS or a combination product of an ICS/LABA. Additionally, recipients are required to have one more prescription claim for ICS or the combination product of ICS/LABA in the follow-up period.

- no ICS: Recipients who filled a prescription for other COPD-related drugs (inhaled longacting beta-agonists, anticholinergics (ipratropium or combination product of ipratropium and albuterol), short-acting beta-agonists, or methylxanthines) but did not fill a prescription for ICS during entire study period. Recipients in the non-ICS group without long-acting beta-agonists were required to have at least two prescriptions of short-acting bronchodilators in the pre-index period of which at least one was for ipratropium. This was done to avoid the possibility of including recipients with mild COPD who use SABD on an as-needed basis. Additionally, recipients were required to have one more prescription claim for other COPD-related drugs in the follow-up period.

\section{Inclusion criteria:}

- Recipients with at least one hospitalization, ER visit or at least two outpatient encounters in the pre-index period with a primary or secondary diagnosis of COPD (ICD-9: 491.xx, 492.xx, 496.xx).

- Recipients at least 35 years at the index date.

- Recipients had to be continuously eligible during the year before the index date. Additionally, recipients found to have died during the 12-month follow-up period were required to be continuously eligible till the date of death, and recipients who did not die during the 12-month follow-up were required to be continuously eligible till the end of the 12-month follow-up period.

\section{Exclusion criteria:}

- Recipients who died within 30 days post-index date were excluded to avoid recipients with very severe COPD.

- Recipients with a diagnosis of respiratory cancer, cystic fibrosis, fibrosis due to tuberculosis, and bronchiectasis during the study period were excluded to avoid misdiagnosis. 
- Recipients over 64 years of age and those with Medicare eligibility were excluded because of insufficient capture of claims.

- Managed care recipients were excluded as managed care recipients do not have all their utilization information in the claims database.

Study design and outcome measure: A historical cohort design was used for objective 9 as demonstrated in figure 17. The outcome was death, and recipients were followed for a period of 12 months until they died or till the end of 12-month follow-up period, whichever occurred first. Additionally, the presence of a severe exacerbation before the date of death or end of follow-up was assessed. Exacerbations were counted 14 days after the index date to avoid protopathic bias i.e. the bias that results when the exposure occurs as a direct result of the outcome. Thus, if an exacerbation was the reason that an ICS was prescribed, this might inadvertently increase the number of exacerbations for the group being prescribed a drug with an inhaled corticosteroid component.

FIGURE 17: Historical cohort design to determine differences in mortality

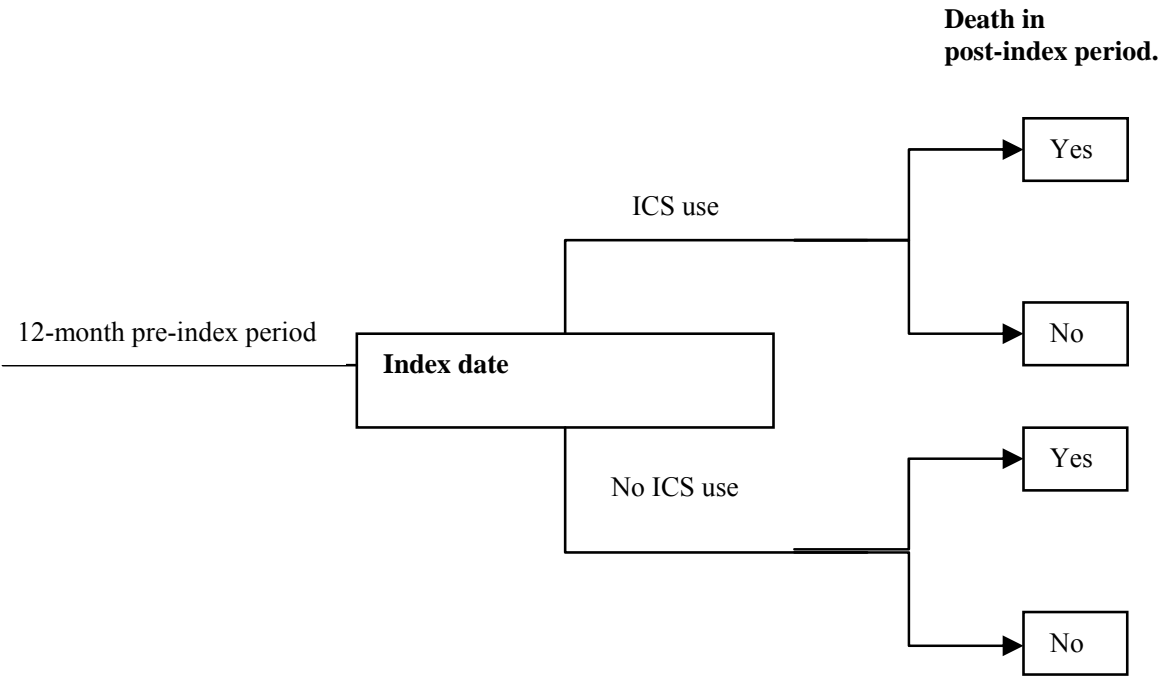




\section{Analyses for research objective 9:}

Objective 9 attempts to understand if any relationship found between ICS use and mortality may be mediated by the impact of ICS on severe exacerbations. Hence, the impact of ICS on mortality as well as on severe exacerbations in the follow-up period was assessed. Description of the study sample in the pre-index period was done using descriptive statistics. Univariate differences in the proportion of those who died and those who experienced a severe exacerbation were analyzed using chi-square tests, and time to death and time to severe exacerbation was analyzed using the log-rank test.

Similar to the analytical approach for part 2 of Phase II, propensity scores were used to account for selection bias in the analyses for research objective 9. However, the matching technique was not used; rather the propensity score was used as a covariate in the regression models (D'Agostino, Jr., 1998). There was a twofold reason for this approach: one was to conserve sample size; and the second was to permit a mediation analysis that can only be done by assessing changes in coefficients of the equations. The approach for regression adjustment involves using the raw propensity score or the propensity score quintile as a covariate in the model. The propensity score quintile for each person is obtained by dividing the study sample into five equally sized strata based on quintiles of the propensity score distribution. The quintile rather than the raw score was used since it permitted one to test whether balance had been achieved in the covariates between the two groups. This was done by conducting two-way ANOVAs using the drug therapy variable (ICS vs. no ICS), propensity score quintile, and interaction of propensity score quintile and drug therapy variable for the continuous variables, and Cochran-Mantel Haenzel tests for the categorical variables (D'Agostino, Jr., 1998).

Survival analysis techniques were used to determine differences in time to death and time to a severe exacerbation. The proportional hazards assumption was tested using the global test of proportional hazards, and the assumption was satisfied. Consequently, a Coxproportional hazards regression was used to determine differences in time to death and time to a severe exacerbation controlling for propensity score quintile and recipient's state. Although state was included in the propensity score models, it was included in the Cox proportional hazards model due to differences in mortality rates between the two states (Hoyert DL, Heron MP, Murphy SL, \& Kung H, 2006). A mediation analysis as suggested 
by Baron and Kenny (MacKinnon, 1994) was originally proposed provided the above analyses demonstrated that ICS impacted both risk of mortality and severe exacerbation. However, as no relationship between use of ICS and severe exacerbation was found, no mediation analysis was conducted. 


\section{CHAPTER 4: RESUlTS \& DisCUSSION}

Chapter 4 details the results and discussion of each objective of the study. As each phase of the study involved different extraction criteria based on the study design, different subsets of recipients were obtained for each objective. Accordingly, the results of each phase are preceded by the resultant extraction process from the raw data.

\section{Phase I}

Phase I of the study involved the determination of costs incurred by recipients with COPD during a year, and claims data for the year 2002 were used to achieve this goal. Table 8 outlines the sample extraction process depending on the study design mentioned in Chapter 3. The process follows the order in which the inclusion criteria were applied.

\section{TABLE 8: Sample extraction for Phase I}

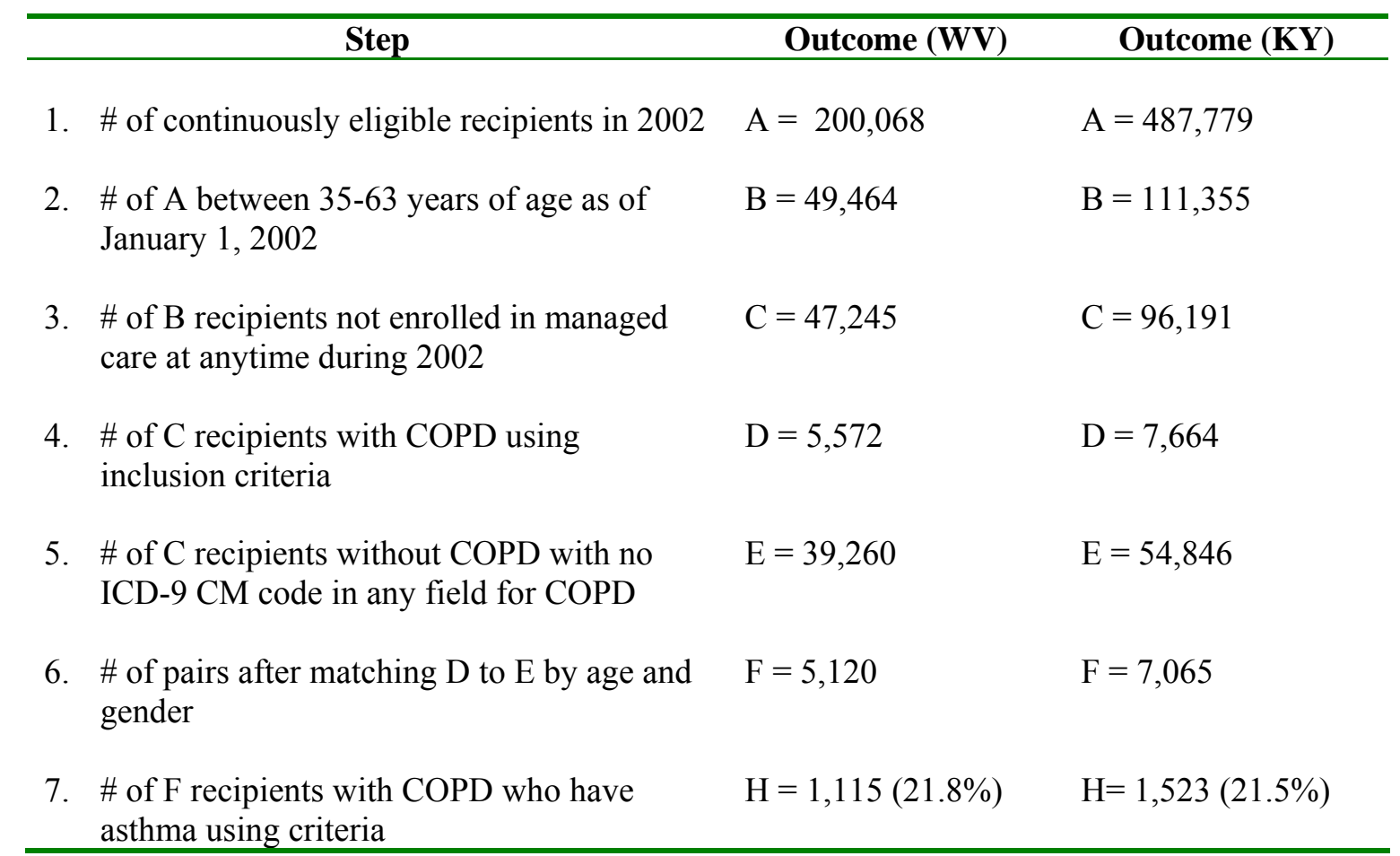

Research Objective 1: To estimate mean incremental costs incurred due to COPD by recipients enrolled in West Virginia Medicaid and Kentucky Medicaid.

The extraction process identified 5,572 and 7,664 recipients with COPD in WV and KY Medicaid, respectively. Each of these recipients were matched by exact age and gender to a 
recipient identified as not having COPD. A total of 5,120 and 7,065 matched pairs were obtained in WV and KY Medicaid, respectively (Table 8). Both states had a similar age and gender distribution. The average age of a recipient with COPD was 52 years in WV and 51 years in KY, and both states had a majority of females (Table 9: 60\% in WV and 64\% in $\mathrm{KY}$ ). Table 9 presents the characteristics of recipients with and without COPD in WV and KY Medicaid. Of recipients with race information, 95 percent were white in both states with a slightly higher proportion for recipients with COPD compared to those without COPD (Table 9_WV: $96.4 \%$ vs. 94.3\%; Table 9_KY: 96.7\% vs. 93.1\%). A slightly higher but significant proportion of recipients with COPD resided in counties with a smoking prevalence rate of at least $31 \%$ compared to those without COPD in both states (Table 9_WV: $24.1 \%$ vs. $22.7 \%$; Table 9_KY: $89.3 \%$ vs. $86.0 \%$ ). Recipients with COPD in both states had a significantly higher D'Hoore CCI index with $50 \%$ of the sample having an index of 2.0 vs 1.0 for non-COPD recipients. Similar patterns were seen comparing the number of chronic conditions. 
TABLE 9: Characteristics of recipients with and without COPD in WV and KY Medicaid

\begin{tabular}{|c|c|c|c|c|c|c|}
\hline \multirow[t]{2}{*}{ Characteristics } & \multicolumn{3}{|c|}{$\begin{array}{l}\text { WV Medicaid } \\
(\mathrm{N}=10,240)\end{array}$} & \multicolumn{3}{|c|}{$\begin{array}{c}\text { KY Medicaid } \\
(\mathbf{N}=\mathbf{1 4 , 1 3 0 )}\end{array}$} \\
\hline & $\begin{array}{c}C O P D \\
(N=5,120)\end{array}$ & $\begin{array}{c}\text { non-COPD } \\
(N=5,120)\end{array}$ & $\begin{array}{c}\text { Test } \\
\text { Statistic } \\
\text { (p-value) }\end{array}$ & $\begin{array}{c}C O P D \\
(N=7,065)\end{array}$ & $\begin{array}{c}\text { non-COPD } \\
(N=7,065)\end{array}$ & $\begin{array}{c}\text { Test } \\
\text { Statistic } \\
\text { (p-value) }\end{array}$ \\
\hline \multicolumn{7}{|l|}{$\begin{array}{l}\text { Demographic } \\
\text { Race, \% (n) }\end{array}$} \\
\hline White & $\begin{array}{l}96.4 \% \\
(4,861)\end{array}$ & $\begin{array}{l}94.3 \% \\
4,731)\end{array}$ & ${ }^{1}(0.000)^{*}$ & $\begin{array}{l}96.7 \% \\
(5,965)\end{array}$ & $\begin{array}{l}93.1 \% \\
(5,778)\end{array}$ & ${ }^{1}(0.000)^{*}$ \\
\hline $\begin{array}{l}\text { Countysmokerate } \\
>>=31 \%\end{array}$ & $\begin{array}{l}24.1 \% \\
(1,236)\end{array}$ & $\begin{array}{l}22.7 \% \\
(1,164)\end{array}$ & ${ }^{1}(0.000)^{*}$ & $\begin{array}{l}89.3 \% \\
(6,311)\end{array}$ & $\begin{array}{l}86.0 \% \\
(6,076)\end{array}$ & ${ }^{1}(0.000)^{*}$ \\
\hline $\begin{array}{l}\text { Overall } \\
\text { comorbidity } \\
\text { D'Hoore-CCI }^{\text {a }}\end{array}$ & & & & & & \\
\hline $\begin{array}{r}\text { Mean (SD) } \\
\text { Median }\end{array}$ & $\begin{array}{c}2.4(2.4) \\
2.0\end{array}$ & $\begin{array}{c}1.4(1.9) \\
1.0\end{array}$ & $\begin{array}{l}-23.82^{2} \\
(0.000)^{*}\end{array}$ & $\begin{array}{c}2.2(2.3) \\
2.0\end{array}$ & $\begin{array}{c}1.2(1.7) \\
1.0\end{array}$ & $\begin{array}{l}-28.95^{2} \\
(0.000)^{*}\end{array}$ \\
\hline $\begin{array}{l}\text { Number of other } \\
\text { chronic } \\
\text { conditions }^{b}\end{array}$ & & & & & & \\
\hline Mean $(\mathrm{SD})$ & $2.1(1.4)$ & $1.6(1.3)$ & $-20.33^{2}$ & $2.0(1.4)$ & $1.4(1.3)$ & $-27.68^{2}$ \\
\hline Median & 2.0 & 1.0 & $(0.000)^{*}$ & 2.0 & 0.0 & $(0.000) *$ \\
\hline
\end{tabular}

$* \mathrm{p}<0.05$

${ }^{\text {a }}$ Excludes COPD

${ }^{\mathrm{b}}$ Includes hypercholesterolemia, hypertension, other heart disease, AIDS, rheumatoid arthritis, osteoarthritis, gall bladder disease, depression and anxiety, and schizophrenia.

${ }^{1} \mathrm{McNemar}$ test, test statistic not available; ${ }^{2}$ Wilcoxon signed rank test statistic 
Comparison of mean total healthcare utilization and costs between recipients with and without COPD in WV and KY Medicaid

Tables 10 and 11 compare the mean total healthcare use and costs of recipients with and without COPD in WV and KY Medicaid, respectively. Almost half of the recipients with COPD had at least one hospitalization visit compared to those without COPD in both states (Table 10_WV: $47.7 \%$ vs. $16.3 \%$; Table $11 \_$KY: $46.3 \%$ vs. $13.0 \%$ ). The number of visits per recipient was also significantly higher in recipients with COPD (Table 10_WV: 0.98 vs. 0.28 ; Table 11_KY: 1.12 vs. 0.22 ). Similar trends were seen for proportions with versus without COPD with at least one ER visit (Table 10_WV: 61.5\% vs. 40.1\%; Table 11_KY: $59.4 \%$ vs. $32.3 \%$ ) and mean number of ER visits (Table 10_WV: 2.31 vs. 1.04; Table 11_KY: 2.33 vs. 0.81 ) in both states. A significantly higher proportion of recipients with COPD had at least one physician visit in both states (Table 10_WV: 90.3\% vs. 79.2\%; Table 11_KY $94.0 \%$ vs. $77.4 \%$ ) with a significantly higher mean number of physician visits (Table 10_WV: 9.08 vs. 5.53; Table 11_KY: 11.75 vs. 6.25) compared to those without COPD. Recipients with COPD also had a significantly higher average number of prescription fills compared to those without COPD in both states, with KY Medicaid recipients filling more prescriptions than WV Medicaid recipients (Table 10_WV:78.91 vs. 50.27; Table 11_KY: 99.18 vs. 67.42 ). Overall, less than $5 \%$ of the sample used nursing home care in 2002. Contrary to other healthcare cost components, recipients without COPD in both states incurred significantly higher nursing home costs than recipients with COPD.

The mean incremental total healthcare cost incurred by recipients with COPD was calculated as the difference in mean total healthcare costs incurred by recipients with and without COPD. Thus, recipients with COPD incurred \$6,589 per recipient in WV Medicaid (Table 12). In multivariate analyses after controlling for confounders, recipients with COPD were found to incur almost $74 \%$ higher total healthcare costs compared to recipients without COPD with an incremental cost of $\$ 3,952$ per recipient (Table 12). Recipients with COPD in KY Medicaid incurred 53\% higher total healthcare costs with an incremental cost of $\$ 4,979$ per recipient with COPD (Table 13). For both states, adjusted analyses showed that a majority of the costs for recipients with COPD was due to the higher cost of hospitalizations for recipients with COPD, followed by costs for outpatient encounters, prescription drugs, and ER visits. In both states, nursing home costs were found to be lower for recipients with 
COPD compared to those without COPD; however, this difference was statistically significant only in the KY Medicaid sample. For both states, the maximum percentage difference in costs between recipients with and without COPD was seen for hospitalization and ER costs.

TABLE 10: Total healthcare utilization and costs for recipients with and without COPD in WV Medicaid

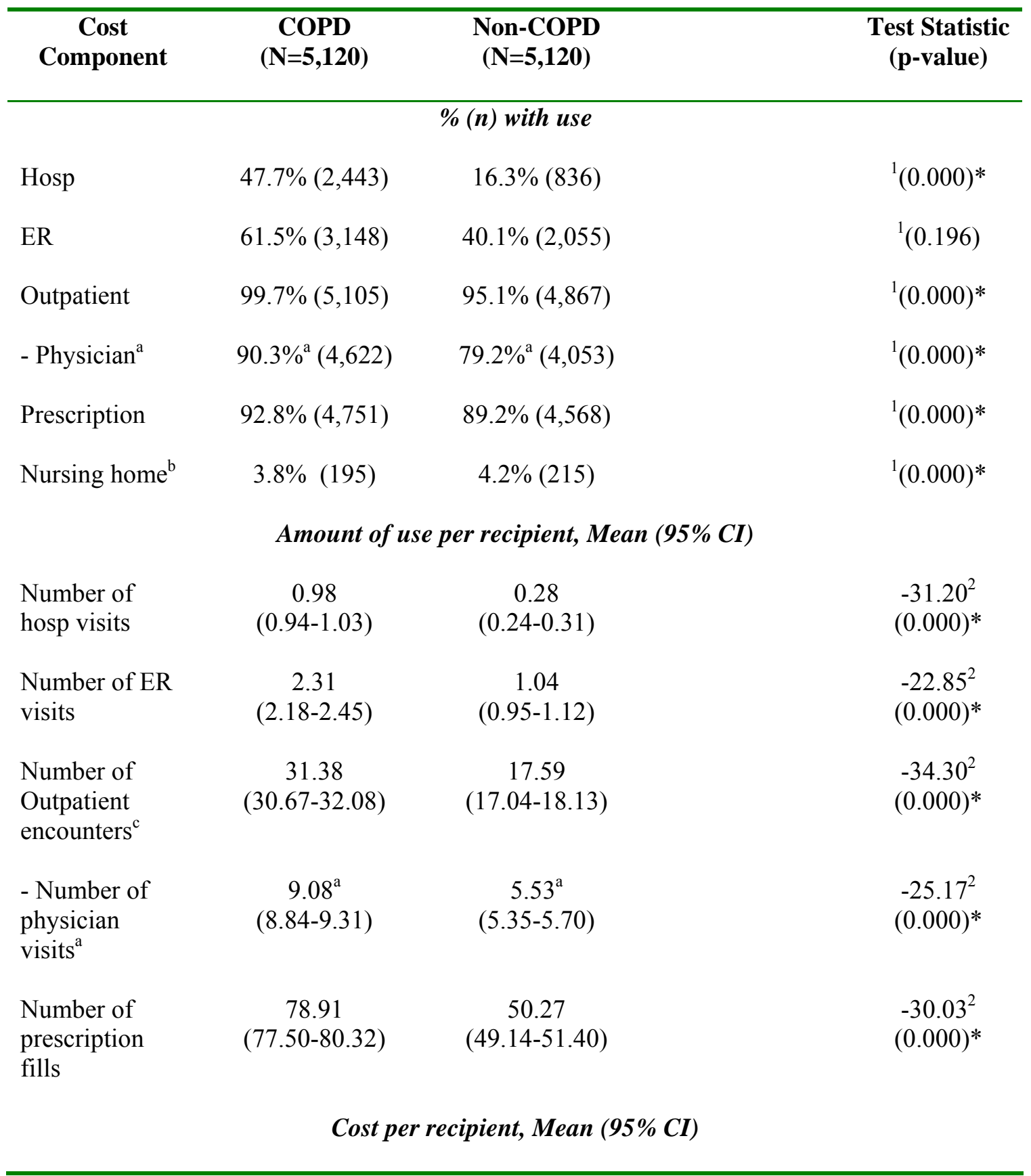




\begin{tabular}{|c|c|c|c|c|}
\hline Hosp & $\begin{array}{c}\$ 5,265 \\
(\$ 4,978-\$ 5,552)\end{array}$ & $\begin{array}{c}\$ 1,538 \\
(\$ 1,370-\$ 1,707)\end{array}$ & $\begin{array}{c}\text { Difference } \\
\$ 3,727 \\
(\$ 3,392-\$ 4,061)\end{array}$ & $\begin{array}{l}-28.85^{2} \\
(0.000)^{*}\end{array}$ \\
\hline ER visit & $\begin{array}{c}\$ 787 \\
(\$ 737-\$ 836)\end{array}$ & $\begin{array}{c}\$ 300 \\
(\$ 275-\$ 324)\end{array}$ & $\begin{array}{c}\$ 487 \\
(\$ 431-\$ 542)\end{array}$ & $\begin{array}{l}-23.70^{2} \\
(0.000)^{*}\end{array}$ \\
\hline Outpatient & $\begin{array}{c}\$ 4,240 \\
(\$ 4,004-\$ 4,477)\end{array}$ & $\begin{array}{c}\$ 2,687 \\
(\$ 2,461-\$ 2,913)\end{array}$ & $\begin{array}{c}\$ 1,554 \\
(\$ 1,226-\$ 1,881)\end{array}$ & $\begin{array}{l}-26.72^{2} \\
(0.000)^{*}\end{array}$ \\
\hline $\begin{array}{l}\text { - Physician } \\
\text { visit }^{\mathrm{a}}\end{array}$ & $\begin{array}{c}\$ 653^{\mathrm{a}} \\
(\$ 616-\$ 691)\end{array}$ & $\begin{array}{c}\$ 362^{\mathrm{a}} \\
(\$ 336-\$ 387)\end{array}$ & $\begin{array}{c}\$ 292 \\
(\$ 246-\$ 337)\end{array}$ & $\begin{array}{l}-20.40^{2} \\
(0.000)^{*}\end{array}$ \\
\hline $\begin{array}{l}\text { Prescription } \\
\text { drugs }\end{array}$ & $\begin{array}{c}\$ 4,058 \\
(\$ 3,962-\$ 4,154)\end{array}$ & $\begin{array}{c}\$ 2,528 \\
(\$ 2,450-\$ 2,606)\end{array}$ & $\begin{array}{c}\$ 1,531 \\
(\$ 1,410-\$ 1,652)\end{array}$ & $\begin{array}{l}-26.45^{2} \\
(0.000)^{*}\end{array}$ \\
\hline Nursing home & $\begin{array}{c}\$ 1,000 \\
(\$ 823-\$ 1,178)\end{array}$ & $\begin{array}{c}\$ 1,709 \\
(\$ 1,445-\$ 1,972)\end{array}$ & $\begin{array}{c}-\$ 709 \\
(-\$ 1,029-\quad-\$ 388)\end{array}$ & $\begin{array}{c}-3.89^{2} \\
(0.000)^{*}\end{array}$ \\
\hline Total costs & $\begin{array}{c}\$ 15,350 \\
(\$ 14,839-\$ 15,861)\end{array}$ & $\begin{array}{c}\$ 8,761 \\
(\$ 8,328-\$ 9,193)\end{array}$ & $\begin{array}{c}\$ 6,590 \\
(\$ 5,916-\$ 7,263)\end{array}$ & $\begin{array}{l}-29.44^{2} \\
(0.000)^{*}\end{array}$ \\
\hline
\end{tabular}

$* \mathrm{p}<0.05$

${ }^{a}$ Physician cost component is a sub-component of the Outpatient cost component.

${ }^{\mathrm{b}}$ Number of nursing home visits cannot be computed from data source

${ }^{c}$ An outpatient encounter is defined as any service for a particular recipient on a unique date that generated a CPT code other than 99281-99285, 99288.

${ }^{1} \mathrm{McNemar}$ test, test statistic not available; ${ }^{2}$ Wilcoxon signed rank test statistic 
TABLE 11: Total healthcare utilization and costs for recipients with and without COPD in KY Medicaid

\begin{tabular}{|c|c|c|c|c|}
\hline $\begin{array}{c}\text { Cost } \\
\text { Component }\end{array}$ & $\begin{array}{c}\text { COPD } \\
(\mathrm{N}=7,065)\end{array}$ & $\begin{array}{c}\text { Non-COPD } \\
(\mathrm{N}=7,065)\end{array}$ & & $\begin{array}{c}\text { Test Statistic } \\
\text { (p-value) }\end{array}$ \\
\hline \multicolumn{5}{|c|}{$\%$ (n) with use } \\
\hline Hosp & $46.3 \%(3,271)$ & $13.0 \%(918)$ & & ${ }^{1}(0.000)^{*}$ \\
\hline ER & $59.4 \%(4,200)$ & $32.3 \%(2,283)$ & & ${ }^{1}(0.000)^{*}$ \\
\hline Outpatient & $99.4 \%(7,024)$ & $96.5 \%(6,817)$ & & ${ }^{1}(0.000)^{*}$ \\
\hline - Physician ${ }^{\mathrm{a}}$ & $94.0 \%{ }^{\mathrm{a}}(6,639)$ & $77.4 \%{ }^{\mathrm{a}}(5,468)$ & & ${ }^{1}(0.000)^{*}$ \\
\hline Prescription & $99.2 \%(7,006)$ & $97.0 \%(6,855)$ & & ${ }^{1}(0.000)^{*}$ \\
\hline Nursing home ${ }^{\mathrm{b}}$ & $3.4 \%(240)$ & $3.0 \%(211)$ & & ${ }^{1}(0.000)^{*}$ \\
\hline \multicolumn{5}{|c|}{ Amount of use per recipient, Mean (95\% CI) } \\
\hline $\begin{array}{l}\text { Number of } \\
\text { hosp visits }\end{array}$ & $\begin{array}{c}1.12 \\
(1.08-1.17)\end{array}$ & $\begin{array}{c}0.22 \\
(0.20-0.24)\end{array}$ & & $\begin{array}{l}-38.81^{2} \\
(0.000)^{*}\end{array}$ \\
\hline $\begin{array}{l}\text { Number of ER } \\
\text { visits }\end{array}$ & $\begin{array}{c}2.33 \\
(2.21-2.45)\end{array}$ & $\begin{array}{c}0.81 \\
(0.76-0.86)\end{array}$ & & $\begin{array}{l}-32.89^{2} \\
(0.000)^{*}\end{array}$ \\
\hline $\begin{array}{l}\text { Number of } \\
\text { Outpatient } \\
\text { encounters }^{c}\end{array}$ & $\begin{array}{c}35.66 \\
(34.66-36.66)\end{array}$ & $\begin{array}{c}29.02 \\
(27.64-30.41)\end{array}$ & & $\begin{array}{l}-27.62^{2} \\
(0.000)^{*}\end{array}$ \\
\hline $\begin{array}{l}\text { - Number of } \\
\text { physician } \\
\text { visits }^{\mathrm{a}}\end{array}$ & $\begin{array}{c}11.75^{\mathrm{a}} \\
(11.54-11.97)\end{array}$ & $\begin{array}{c}6.25^{\mathrm{a}} \\
(6.09-6.41)\end{array}$ & & $\begin{array}{c}-40.03^{2} \\
(0.000)^{*}\end{array}$ \\
\hline $\begin{array}{l}\text { Number of } \\
\text { prescription } \\
\text { fills }\end{array}$ & $\begin{array}{c}99.18 \\
(97.69-100.67)\end{array}$ & $\begin{array}{c}67.42 \\
(66.16-68.68)\end{array}$ & & $\begin{array}{l}-31.74^{2} \\
(0.000)^{*}\end{array}$ \\
\hline \multicolumn{5}{|c|}{ Cost per recipient, Mean (95\% CI) } \\
\hline Hosp & $\begin{array}{c}\$ 4,495 \\
(\$ 4,252-\$ 4,738)\end{array}$ & $\begin{array}{c}\$ 922 \\
(\$ 817-\$ 1,028)\end{array}$ & $\begin{array}{c}\text { Difference } \\
\$ 3,573 \\
(\$ 3,308-\$ 3,837)\end{array}$ & $\begin{array}{l}-37.14^{2} \\
(0.000)^{*}\end{array}$ \\
\hline ER visit & $\begin{array}{c}\$ 858 \\
(\$ 815-\$ 900)\end{array}$ & $\begin{array}{c}\$ 274 \\
(\$ 255-\$ 293)\end{array}$ & $\begin{array}{c}\$ 584 \\
(\$ 538-\$ 630)\end{array}$ & $\begin{array}{l}-33.78^{2} \\
(0.000)^{*}\end{array}$ \\
\hline Outpatient & $\$ 7,283$ & $\$ 8,115$ & $-\$ 831$ & $-34.09^{2}$ \\
\hline
\end{tabular}




\begin{tabular}{|c|c|c|c|c|}
\hline & $(\$ 6,986-\$ 7,580)$ & $(\$ 7,176-\$ 9,053)$ & $(-\$ 1,816-\$ 153)$ & $(0.000)^{*}$ \\
\hline $\begin{array}{l}\text { - Physician } \\
\text { visit }^{\mathrm{a}}\end{array}$ & $\begin{array}{c}\$ 1,024^{\mathrm{a}} \\
(\$ 991-\$ 1,056)\end{array}$ & $\begin{array}{c}\$ 491^{\mathrm{a}} \\
(\$ 471-\$ 510)\end{array}$ & $\begin{array}{c}\$ 533 \\
(\$ 495-\$ 571)\end{array}$ & $\begin{array}{l}-37.26^{2} \\
(0.000)^{*}\end{array}$ \\
\hline $\begin{array}{l}\text { Prescription } \\
\text { drugs }\end{array}$ & $\begin{array}{c}\$ 5,303 \\
(\$ 5,198-\$ 5,408)\end{array}$ & $\begin{array}{c}\$ 3,529 \\
(\$ 3,444-\$ 3,614)\end{array}$ & $\begin{array}{c}\$ 1,774 \\
(\$ 1,640-\$ 1,909)\end{array}$ & $\begin{array}{c}-28.45^{2} \\
(0.000)^{*}\end{array}$ \\
\hline Nursing home & $\begin{array}{c}\$ 631 \\
(\$ 525-\$ 737)\end{array}$ & $\begin{array}{c}\$ 1,240 \\
(\$ 1,014-\$ 1,467)\end{array}$ & $\begin{array}{c}-\$ 610 \\
(-\$ 859--\$ 361)\end{array}$ & $\begin{array}{c}-2.66^{2} \\
(0.008)^{*}\end{array}$ \\
\hline Total costs & $\begin{array}{c}\$ 18,569 \\
(\$ 18,041-\$ 19,098)\end{array}$ & $\begin{array}{c}\$ 14,080 \\
(\$ 13,091-\$ 15,069)\end{array}$ & $\begin{array}{c}\$ 4,489 \\
(\$ 3,368-\$ 5,610)\end{array}$ & $\begin{array}{l}-36.01^{2} \\
(0.000)^{*}\end{array}$ \\
\hline
\end{tabular}

$* \mathrm{p}<0.05$

${ }^{a}$ Physician cost component is a sub-component of the Outpatient cost component.

${ }^{\mathrm{b}}$ Number of nursing home visits cannot be computed from data source

${ }^{\mathrm{c}}$ An outpatient encounter is defined as any service for a particular recipient on a unique date that generated a CPT code other than 99281-99285, 99288.

${ }^{1} \mathrm{McNemar}$ test, test statistic not available; ${ }^{2} \mathrm{Wilcoxon}$ signed rank test statistic 
TABLE 12: Mean incremental total healthcare costs incurred by recipients with COPD [Total healthcare costs incurred by recipients with COPD - Total healthcare costs incurred by recipients without COPD] in WV Medicaid

\begin{tabular}{|c|c|c|c|c|}
\hline Cost & Unadjusted & \multicolumn{3}{|c|}{ Adjusted $^{\mathrm{a}}$} \\
\hline & $\begin{array}{c}\text { Difference in } \\
\text { cost per recipient }\end{array}$ & $\begin{array}{c}\text { Difference in } \\
\text { cost per recipient }\end{array}$ & $\begin{array}{l}\text { Percentage difference } \\
\text { in costs }{ }^{b}\end{array}$ & $\begin{array}{c}\text { Model } \\
\text { Details: see } \\
\text { Appendix } \\
\text { table }\end{array}$ \\
\hline Hosp & $\$ 3,727^{*}$ & $\$ 2,424 *$ & $+178.2 \% *$ & A1 \\
\hline ER visit & $\$ 487^{*}$ & $\$ 218^{*}$ & $+80.7 \% *$ & $\mathrm{~A} 2$ \\
\hline Outpatient & $\$ 1,554^{*}$ & $\$ 1,111^{*}$ & $+63.8 \% *$ & A3 \\
\hline $\begin{array}{l}\text { - Physician } \\
\text { visit }^{\mathrm{c}}\end{array}$ & $\$ 292^{\mathrm{c} *}$ & $\$ 127^{\mathrm{c} *}$ & $+26.2 \%{ }^{\mathrm{c} *}$ & A4 \\
\hline $\begin{array}{l}\text { Prescription } \\
\text { drugs }\end{array}$ & $\$ 1,531 *$ & $\$ 1,026^{*}$ & $+36.5 \% *$ & A5 \\
\hline Nursing home & - \$709* & $-\$ 536$ & $-35.7 \%$ & A6 \\
\hline Total costs & $\$ 6,590 *$ & $\$ 3,952^{d_{*}}$ & 73.5\%* & A7 \\
\hline
\end{tabular}

$* \mathrm{p}<0.05$

${ }^{a}$ Covariates include race, countysmokingrate, D'Hoore-CCI, and number of other chronic conditions.

${ }^{\mathrm{b}}$ (Cost per COPD recipient - Cost per non-COPD recipient)/Cost per non-COPD recipient. + indicates that COPD recipients have higher costs than those without COPD. * indicates that the difference was statistically significant.

${ }^{\mathrm{c}}$ Physician cost component is a sub-component of the Outpatient cost component.

${ }^{\mathrm{d}}$ Total adjusted cost may not reflect the sum of individual cost components because estimates are obtained from individual regression models for each component. 
TABLE 13: Mean incremental total healthcare costs incurred by recipients with COPD [Total healthcare costs incurred by recipients with COPD - Total healthcare costs incurred by recipients without COPD] in KY Medicaid

\begin{tabular}{|c|c|c|c|c|}
\hline Cost & Unadjusted & & Adjusted $^{\mathrm{a}}$ & \\
\hline Hosp & $\begin{array}{c}\text { Difference in } \\
\text { cost per recipient } \\
\$ 3,573 *\end{array}$ & $\begin{array}{c}\text { Difference in } \\
\text { cost per recipient }\end{array}$ & $\begin{array}{l}\text { Percentage difference } \\
\text { in costs } \\
+280.4 \% *\end{array}$ & $\begin{array}{l}\text { Details of } \\
\text { Model in } \\
\text { Appendix } \\
\text { A8 }\end{array}$ \\
\hline ER visit & $\$ 584 *$ & $\$ 381 *$ & $+146.1 \% *$ & A9 \\
\hline Outpatient & $-\$ 831^{*}$ & $\$ 1,785^{*}$ & $+52.9 \% *$ & A10 \\
\hline $\begin{array}{l}\text { - Physician } \\
\text { visit }^{\mathrm{c}}\end{array}$ & $\$ 533^{\mathrm{c} *}$ & $\$ 304 *$ & $+57.9 \% *$ & A11 \\
\hline $\begin{array}{l}\text { Prescription } \\
\text { drugs }\end{array}$ & $\$ 1,774^{*}$ & $\$ 945^{*}$ & $+24.2 \% *$ & A12 \\
\hline Nursing home & $-\$ 610^{*}$ & $-\$ 549 *$ & $-45.6 \% *$ & A13 \\
\hline Total costs & $\$ 4,489 *$ & $\$ 4,979^{d}$ & $+53.1 \%$ & A14 \\
\hline
\end{tabular}

$* \mathrm{p}<0.05$

a Covariates include race, countysmokingrate, D'Hoore-CCI, and number of other chronic conditions.

${ }^{\mathrm{b}}$ (Cost per COPD recipient - Cost per non-COPD recipient)/Cost per non-COPD recipient. + indicates that COPD recipients have higher costs than those without COPD. * indicates that the difference was statistically significant.

${ }^{\mathrm{c}}$ Physician cost component is a sub-component of the Outpatient cost component.

${ }^{\mathrm{d}}$ Total adjusted cost may not reflect the sum of individual cost components because estimates are obtained from individual regression models for each component. 
Mean COPD-related utilization and costs in relation to mean total healthcare costs for recipients with COPD in $W V \& K Y$ Medicaid

Tables 14 and 15 compare the mean COPD-related utilization and costs for recipients with COPD in relation to their mean total healthcare costs in WV and KY Medicaid, respectively. Both states showed lower rates of COPD-related use and costs for recipients with COPD compared to the recipients' total healthcare use and costs. The mean COPDrelated costs for recipients with COPD in WV Medicaid was $\$ 4,999$, and constituted approximately 35 percent of total healthcare costs. The mean COPD related costs for recipients with COPD in KY Medicaid was higher $(\$ 6,346)$ but the proportion of total healthcare costs $(35 \%)$ was similar to WV Medicaid. In both states, the average cost for a COPD-related hospitalization consumed a greater proportion of the average cost for total healthcare hospitalizations compared to other cost components. Recipients in KY Medicaid had a higher mean number of COPD-related hospitalizations compared to those in WV Medicaid.

Table 16 presents the proportion of Medicaid recipients with COPD in both states using each of eight COPD-related drug classes. A higher proportion of recipients with COPD in WV Medicaid had no COPD-related prescription drug use compared to recipients with COPD in KY Medicaid (11\% vs. $3 \%$ ). The use of all drug categories was higher in KY Medicaid than that in WV Medicaid. Anticholinergics, inhaled long-acting beta-agonists, inhaled corticosteroids alone or in combination with long-acting beta-agonists, and methylxanthines are used as maintenance drug therapy in COPD. The use of ipratropium, the gold standard COPD drug treatment, alone or in combination with albuterol, occurred in 53 percent of recipients with COPD in KY Medicaid compared to 43 percent in WV Medicaid. The use of the other maintenance drug therapies occurred in less than half of the study sample in both states. Almost 80 to 90 percent were found to be using antibiotics indicated for respiratory infections. Use of short-acting beta-agonists and oral corticosteroids was also high.

The use and costs of home oxygen therapy were also calculated. Costs for home oxygen therapy can be included in either the hospitalization, ER, or outpatient components based on the nature of billing claims in the U.S. Hence, costs of home oxygen therapy were calculated using all claims with HCPCS codes for oxygen therapy. Thirty-two percent of the recipients 
with COPD in WV Medicaid used home oxygen therapy incurring an average cost of $\$ 516$. Corresponding numbers in KY Medicaid were 26.2 percent and $\$ 622$.

TABLE 14: Total healthcare and COPD-related ${ }^{\mathrm{a}}$ utilization and cost per recipient for recipients with COPD $(N=5,120)$ in WV Medicaid

\begin{tabular}{|c|c|c|c|}
\hline Cost Component & Total healthcare & COPD-related & \\
\hline \multicolumn{4}{|c|}{$\%$ (n) with use } \\
\hline Hosp & $47.7 \%(2,443)$ & $16.5 \%(1,688)$ & \\
\hline ER & $61.5 \%(3,148)$ & $15.4 \%(1,266)$ & \\
\hline Outpatient & $99.7 \%(5,105)$ & $46.0 \%(4,713)$ & \\
\hline - Physician ${ }^{\mathrm{b}}$ & $90.3 \% \%^{b}(4,622)$ & $63.3 \%$ b $(3,239)$ & \\
\hline Prescription & $92.8 \%(4,751)$ & $89.3 \%(4,572)$ & \\
\hline \multicolumn{4}{|c|}{ Amount of use per recipient, Mean (95\% CI) } \\
\hline Number of hosp visits & $\begin{array}{c}0.98 \\
(0.94-1.03)\end{array}$ & $\begin{array}{c}0.16 \\
(0.55-0.52)\end{array}$ & \\
\hline Number of ER visits & $\begin{array}{c}2.31 \\
(2.18-2.45)\end{array}$ & $\begin{array}{c}0.54 \\
(0.50-0.58)\end{array}$ & \\
\hline $\begin{array}{l}\text { Number of Outpatient } \\
\text { encounters }\end{array}$ & $\begin{array}{c}31.38 \\
(30.67-32.08)\end{array}$ & $\begin{array}{c}8.52 \\
(8.24-8.80)\end{array}$ & \\
\hline $\begin{array}{l}\text { - Number of physician } \\
\text { visits }^{b}\end{array}$ & $\begin{array}{c}9.08^{\mathrm{b}} \\
(8.84-9.31)\end{array}$ & $\begin{array}{c}2.11^{\mathrm{b}} \\
(2.03-2.18)\end{array}$ & \\
\hline $\begin{array}{l}\text { Number of prescription } \\
\text { fills }\end{array}$ & $\begin{array}{c}78.91 \\
(77.50-80.32)\end{array}$ & $\begin{array}{c}16.16 \\
(15.71-16.61)\end{array}$ & \\
\hline \multicolumn{4}{|c|}{ Cost per recipient, Mean (95\% CI) } \\
\hline & & & $\begin{array}{c}\% \text { of total } \\
\text { healthcare related } \\
\text { to COPD }\end{array}$ \\
\hline Hosp & $\begin{array}{c}\$ 5,265 \\
(\$ 4,978-\$ 5,552)\end{array}$ & $\begin{array}{c}\$ 2,860 \\
(\$ 2,656-\$ 3,064)\end{array}$ & $54.3 \%$ \\
\hline ER visit & $\begin{array}{c}\$ 787 \\
(\$ 737-\$ 836)\end{array}$ & $\begin{array}{c}\$ 228 \\
(\$ 208-\$ 248)\end{array}$ & $29.0 \%$ \\
\hline
\end{tabular}


Outpatient

- Physician visit ${ }^{\mathrm{b}}$

Prescription drugs

Total costs excluding nursing home costs
$\$ 4,240$

$(\$ 4,004-\$ 4,477)$

$\$ 653^{\mathrm{b}}$

(\$616-\$691)

$\$ 4,058$

$(\$ 3,962-\$ 4,154)$

$\$ 14,350$

(\$13,886-

$\$ 14,814)$
$23.9 \%$

(\$964-\$1,061)

$\$ 155^{b}$
$\$ 144-\$ 165)$

$\$ 899$

$22.2 \%$

(\$869-\$928)

\section{$\$ 4,999$}

$34.8 \%$

${ }^{a}$ COPD-related costs were computed from the paid amounts of the medical claims with a primary or secondary diagnosis of COPD, and prescription claims of COPD-related drug classes. COPD-related drug classes include: anticholinergics(ipratropium, ipratropium/albuterol), inhaled long-acting beta-agonists, short-acting beta-agonists, inhaled corticosteroids, inhaled corticosteroid/long-acting beta-agonist combination, methylxanthines, oral corticosteroids, and antibiotics for respiratory infections. COPD-related nursing home costs could not be calculated due to the absence of diagnoses codes on nursing home claims.

${ }^{\mathrm{b}}$ Physician cost component is a sub-component of the Outpatient cost component.

${ }^{c}$ An outpatient encounter is defined as any service for a particular recipient on a unique date that generated a CPT code other than 99281-99285, 99288. 


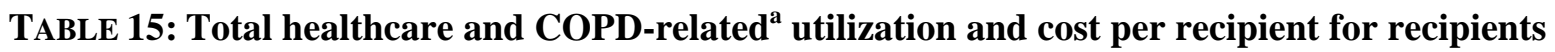
with COPD $(\mathrm{N}=7,065)$ in $\mathrm{KY}$ Medicaid

\begin{tabular}{|c|c|c|c|}
\hline Cost Component & Total healthcare & COPD-related & \\
\hline \multicolumn{4}{|c|}{$\%$ (n) with use } \\
\hline Hosp & $46.3 \%(3,271)$ & $34.0 \%(2,404)$ & \\
\hline ER & $59.4 \%(4,200)$ & $29.6 \%(2,092)$ & \\
\hline Outpatient & $99.4 \%(7,024)$ & $83.7 \%(5,914)$ & \\
\hline - Physician ${ }^{\mathrm{b}}$ & $94.0 \%{ }^{\mathrm{b}}(6,639)$ & $75.2 \% \%^{\mathrm{b}}(5,315)$ & \\
\hline Prescription & $99.2 \%(7,006)$ & $96.7 \%(6,832)$ & \\
\hline \multicolumn{4}{|c|}{ Amount of use per recipient, Mean (95\% CI) } \\
\hline Number of hosp visits & $\begin{array}{c}1.12 \\
(1.08-1.17)\end{array}$ & $\begin{array}{c}0.63 \\
(0.60-0.67)\end{array}$ & \\
\hline Number of ER visits & $\begin{array}{c}2.33 \\
(2.21-2.45)\end{array}$ & $\begin{array}{c}0.52 \\
(0.49-0.56)\end{array}$ & \\
\hline $\begin{array}{l}\text { Number of Outpatient } \\
\text { encounters }^{c}\end{array}$ & $\begin{array}{c}35.66 \\
(34.66-36.66)\end{array}$ & $\begin{array}{c}10.08 \\
(9.46-10.69)\end{array}$ & \\
\hline $\begin{array}{l}\text { - Number of physician } \\
\text { visits }^{\mathrm{b}}\end{array}$ & $\begin{array}{c}11.75^{\mathrm{b}} \\
(11.54-11.97)\end{array}$ & $\begin{array}{c}3.05^{\mathrm{b}} \\
(2.97-3.13)\end{array}$ & \\
\hline $\begin{array}{l}\text { Number of prescription } \\
\text { fills }\end{array}$ & $\begin{array}{c}99.18 \\
(97.69-100.67)\end{array}$ & $\begin{array}{c}22.55 \\
(22.08-23.01)\end{array}$ & \\
\hline \multicolumn{4}{|c|}{ Cost per recipient, Mean (95\% CI) } \\
\hline & & & $\begin{array}{l}\% \text { of total } \\
\text { healthcare } \\
\text { related to } \\
\text { COPD }\end{array}$ \\
\hline Hosp & $\begin{array}{c}\$ 4,495 \\
(\$ 4,252-\$ 4,738)\end{array}$ & $\begin{array}{c}\$ 2,404 \\
(\$ 2,249-\$ 2,560)\end{array}$ & $53.5 \%$ \\
\hline ER visit & $\begin{array}{c}\$ 858 \\
(\$ 815-\$ 900)\end{array}$ & $\begin{array}{c}\$ 253 \\
(\$ 234-\$ 271)\end{array}$ & $29.5 \%$ \\
\hline Outpatient & $\begin{array}{c}\$ 7,283 \\
(\$ 6,986-\$ 7,580)\end{array}$ & $\begin{array}{c}\$ 2,368 \\
(\$ 2,266-\$ 2,469)\end{array}$ & $32.5 \%$ \\
\hline
\end{tabular}




\begin{tabular}{lccc}
\hline - Physician visit $^{\mathrm{b}}$ & $\begin{array}{c}\$ 1,024^{\mathrm{b}} \\
(\$ 991-\$ 1,056)\end{array}$ & $\begin{array}{c}\$ 287^{\mathrm{b}} \\
(\$ 275-\$ 299)\end{array}$ & $28 . \%^{\mathrm{b}}$ \\
Prescription drugs & $\$ 5,303$ & $\$ 1,321$ & $24.9 \%$ \\
& $(\$ 5,198-\$ 5,408)$ & $(\$ 1,289-\$ 1,354)$ & \\
$\begin{array}{l}\text { Total costs excluding } \\
\text { nursing home costs }\end{array}$ & $\mathbf{\$ 1 7 , 9 3 9}$ & $\mathbf{\$ 6 6 , 3 4 6}$ & $\mathbf{3 5 . 4 \%}$ \\
\end{tabular}

${ }^{a}$ COPD-related costs were computed from the paid amounts of the medical claims with a primary or secondary diagnosis of COPD, and prescription claims of COPD-related drug classes. COPD-related drug classes include: anticholinergics(ipratropium, ipratropium/albuterol), inhaled long-acting beta-agonists, short-acting beta-agonists, inhaled corticosteroids, inhaled corticosteroid/long-acting beta-agonist combination, methylxanthines, oral corticosteroids, and antibiotics for respiratory infections. COPD-related nursing home costs could not be calculated due to the absence of diagnoses codes on nursing home claims.

${ }^{\mathrm{b}}$ Physician cost component is a sub-component of the Outpatient cost component.

${ }^{\mathrm{c}}$ An outpatient encounter is defined as any service for a particular recipient on a unique date that generated a CPT code other than 99281-99285, 99288. 
TABLE 16: Utilization of COPD-related prescription drugs and home oxygen therapy for recipients with COPD in WV $(N=5,120)$ and $K Y(N=7,065)$ Medicaid

\begin{tabular}{lcc}
\hline \multicolumn{1}{c}{ Prescription drug category } & WV Medicaid & KY Medicaid \\
\hline $\begin{array}{l}\text { 1. Any drug use } \\
\text { 2. Non-COPD related prescription drug use }\end{array}$ & $92.8 \%(4,751)$ & $99.2 \%(7,006)$ \\
3. COPD-related prescription drug use & $92.3 \%(4,724)$ & $98.8 \%(5,108)$ \\
a. Anticholinergics (Ipratropium, & $89.3 \%(4,572)$ & $96.7 \%(6,832)$ \\
$\quad$ Ipratropium/Albuterol combination) & $43.6 \%(2,233)$ & $53.4 \%(3,775)$ \\
b. Inhaled Long-acting beta-agonists & $16.5 \%(845)$ & $22.0 \%(1,554)$ \\
c. Short-acting beta-agonists (all doseforms) & $58.9 \%(3,017)$ & $70.1 \%(4,952)$ \\
d. Inhaled corticosteroids & $27.9 \%(1,430)$ & $35.0 \%(2,473)$ \\
e. Inhaled corticosteroids/Long-acting beta-agonist & $25.6 \%(1,312)$ & $24.7 \%(1,744)$ \\
$\quad$ combination (Fluticasone/Salmeterol) & & \\
f. Methylxanthines & $21.1 \%(1,084)$ & $27.1 \%(1,916)$ \\
g. Oral corticosteroids & $42.2 \%(2,160)$ & $49.5 \%(3,494)$ \\
h. Antibiotics for respiratory infections & $79.0 \%(4,047)$ & $88.6 \%(6,258)$ \\
4. Use of home oxygen therapy & $32.0 \%(1,640)$ & $26.2 \%(1,851)$ \\
\hline
\end{tabular}




\section{Discussion for Research Objective 1:}

Objective 1 estimated three annual cost estimates for recipients with COPD: the total healthcare costs, the COPD-related costs, and the incremental cost due to COPD. The three provide different pieces of information that can be used by policymakers. The total healthcare cost provides the annual average cost incurred by a recipient with COPD for any healthcare use. The COPD-related costs are computed using only claims with a primary and secondary diagnosis of $\mathrm{COPD}$, and prescription drugs indicated for treating COPD. The total healthcare cost is obviously not an accurate representation of how much a recipient with COPD incurs due to COPD. The estimate of COPD-related costs as obtained from claims data may under-estimate the costs incurred due to COPD. The reason is that costs incurred by conditions resulting from a consequence of COPD are not captured. For instance, congestive heart failure can be considered a COPD-related comorbidity, and may be exacerbated in the presence of COPD or could also result from COPD. However, attributing costs to different conditions as a result of COPD runs the risk of making untenable assumptions, and consequently misestimating costs. Therefore, an incremental approach that compares the costs incurred by recipients with COPD versus without COPD provides a better representation of the costs incurred due to COPD. However, the difference in costs between these two groups should be adjusted for other comorbid conditions and relevant characteristics in multivariate analyses.

The results of objective 1 showed recipients with COPD to incur $\$ 15,350$ to $\$ 18,569$ per person in total healthcare costs in the year 2002 using Medicaid claims data from two states. Only two published studies have computed the total healthcare costs for recipients with COPD. A study conducted by Strassels et al. estimated a person with COPD to cost $\$ 6,500$ in 1987 (Strassels et al., 2001). At that time, hospitalizations accounted for the majority (70\%) of the total mean cost followed by that for outpatient (22\%), while prescription drugs accounted for a mere 8 percent of the total mean COPD expenditures. The other more recent study by Mapel et al. in 1997 estimated an average of \$11,678 with hospitalizations and outpatient encounters accounting for an equivalent proportion of the total mean cost ( 43\%), and prescription drugs accounting for 13.2 percent of the total mean cost (Mapel et al., 2000a). Similar results were seen in the present study with hospitalizations (WV: $34.2 \%$ \& KY: 24.2\%) and outpatient encounters (WV: 27.6\% \& KY: 39.2\%) accounting for the 
majority of costs in both states. Prescription drugs accounted for approximately $26-28$ percent of the total healthcare costs in both states. The increase since the 1987 study in the proportion accounted by prescription drugs this study can be explained by two reasons. For one, at least 50 percent of recipients with COPD have at least one chronic condition and a higher D'Hoore CCI. Several new prescription drugs have been approved since the past decade for most therapeutic categories indicated for chronic conditions, and could explain the increase in non-COPD related prescription drug use. Also, the pharmacological alternatives for treating COPD have increased considerably in the past 5 years with the introduction of long-acting beta-agonists and inhaled corticosteroids for COPD. This is in contrast to Strassels' 1987 study, where only ipratropium was primarily used for COPD.

It is of interest to note that in the two studies by Strassels et al. and Mapel et al., the proportion of costs related to COPD was only $\sim 25 \%$ of the total healthcare costs incurred by recipients with COPD (Strassels et al., 2001; Mapel et al., 2000a). In the present study, COPD-related costs represented 35 percent of the total healthcare costs incurred by recipients with COPD (WV: \$4,999 \& KY: \$6,346). For most cost components, the proportion of the COPD-related cost to the total cost component ranged from 25 percent to 32 percent. However, in the case of hospitalizations, costs for COPD-related hospitalizations comprised almost 50 percent of the costs for all hospitalizations for recipients with COPD. This finding emphasizes the impact that COPD can have on the hospitalization cost component compared to other cost components.

In the present study, home oxygen therapy was used by 32 percent of the WV Medicaid sample, and by 26 percent of the KY Medicaid sample. The mean cost computed represented approximately 10 percent of the total COPD-related cost in both states. Three studies conducted in the U.S. have provided information on the cost of home oxygen therapy. Ward et al. determined home oxygen therapy to account for 35 percent of total COPD-related direct medical costs in the U.S. in 1996 (Ward et al., 2000). Their exceedingly high estimate may be due to the fact that it is based on the prevalence estimate of 616,000 persons receiving home oxygen therapy in the U.S. in 1996, with the assumption that all have COPD. A recent survey in the U.S. (Confronting COPD survey) found 26 percent of the sample to be using home oxygen therapy, similar to our sample proportions, however, unlike our cost estimates, home oxygen therapy was only $1 \%$ of the total direct medical costs (Halpern et al., 2003). 
The discrepancy maybe attributed to the fact that oxygen use was adjusted by the number of days of use of oxygen therapy (Halpern, Musin, \& Sondhi, 2003). Our estimates are similar to a study by Hilleman et al. where oxygen therapy accounted for 15 to 20 percent of total costs for patients with moderate and severe COPD, respectively (Hilleman et al., 2000).

In the present study, the incremental cost due to COPD was $\$ 3,952$ per recipient in WV Medicaid, and \$4,979 per recipient in KY Medicaid after controlling for demographic characteristics and other comorbid chronic conditions. Our estimates are very similar to the recent cost of COPD study done in the U.S- the Confronting COPD survey, in which the mean direct medical cost was $\$ 4,120$ per patient (Halpern et al., 2003). This study is considered the most comprehensive of all cost-of-illness studies in COPD since it also included undiagnosed patients with COPD whose data are frequently not available in other cost of illness studies including ours. The Confronting COPD survey found hospitalizations to comprise 70 percent of the total direct medical costs with less than 17 percent and 1 percent accounted for by outpatient and prescription drug costs (Halpern et al., 2003). In our study, hospitalizations still comprised the majority $(\sim 50 \%)$, however, outpatient and prescription drugs comprised $\sim 30$ percent and $\sim 20$ percent, respectively of the total incremental cost. The inclusion of undiagnosed patients in the Confronting COPD survey may have reduced the proportion seeking treatment, and consequently the costs of the latter components.

Our study showed that a recipient with COPD cost almost 1.5 to 1.7 times more than a recipient without COPD. Similar estimates were found by Mapel et al. (ratio of 2.0) and Grasso et al. (ratio of 2.4) (Mapel et al., 2000a; Grasso et al., 1998). The greatest difference in costs was due to differences in costs for hospitalizations and ER costs emphasizing the severe nature of the condition. In our study, nursing home costs was the only component where recipients with COPD were found to incur lower costs than non-COPD recipients. Available studies in the literature have not incorporated this cost component, and consequently we have no studies to which we can compare our results. However, the lower cost can probably be attributed to a policy initiative by Medicaid of reducing costs by shifting to home-based care from nursing home care (Wiener, Tilly, \& Alecxih, 2002). Kentucky was one of seven states in the U.S. to participate in a home and community based waiver system for older people and younger adults with physical disabilities, and might 
explain why a significant difference was found in the nursing home cost component as opposed to that seen in the West Virginia sample (Wiener et al., 2002). 
Research Objective 2: To compare mean COPD-related costs among recipients with COPD with and without a concomitant diagnosis of asthma enrolled in West Virginia and Kentucky Medicaid.

Of the recipients with COPD identified in objective 1, 22 percent had concomitant asthma in both states. A demographic distribution of these recipients with COPD by concomitant asthma status is given in Table 17. Recipients with COPD with asthma in both states were on average three years younger than those without asthma. Although statistically significant, this difference is of no practical significance. Recipients with COPD with asthma had a significantly higher proportion of females compared to those without asthma in both states. In WV Medicaid, recipients with COPD with asthma were also significantly more likely to be white compared to those without asthma. No differences were seen by race in KY Medicaid. In terms of overall comorbidity, the two groups had similar D'Hoore CCI (excluding asthma and CHF) and number of chronic conditions. In terms of COPD-related comorbid disorders however, a significantly higher proportion of recipients with COPD with asthma in both states were found to have upper respiratory tract infections, lower respiratory tract infections, allergic rhinitis, other disease of the lung, and disorders of fluid, electrolyte, and acid-base balance compared to recipients with COPD without asthma. The groups did not differ in the proportions having septicemia and CHF, although in KY Medicaid, there was a slightly higher proportion of recipients with COPD with asthma having CHF. 
TABLE 17: Characteristics of recipients with COPD with and without concomitant asthma in WV and KY Medicaid

\begin{tabular}{|c|c|c|c|c|c|c|}
\hline \multirow[t]{2}{*}{ Characteristics } & \multicolumn{3}{|c|}{$\begin{array}{l}\text { WV Medicaid } \\
(\mathrm{N}=5,120)\end{array}$} & \multicolumn{3}{|c|}{$\begin{array}{l}\text { KY Medicaid } \\
\quad(\mathrm{N}=7,065)\end{array}$} \\
\hline & $\begin{array}{c}\text { COPD } \\
\text { with } \\
\text { asthma } \\
(N=1,115)\end{array}$ & $\begin{array}{c}\text { COPD } \\
\text { without } \\
\text { asthma } \\
(N=4,005)\end{array}$ & $\begin{array}{c}\text { Test } \\
\text { Statistic } \\
\text { (p-value) }\end{array}$ & $\begin{array}{c}\text { COPD } \\
\text { with } \\
\text { asthma } \\
(N=1,523)\end{array}$ & $\begin{array}{c}\text { COPD } \\
\text { without } \\
\text { asthma } \\
(N=5,542)\end{array}$ & $\begin{array}{c}\text { Test } \\
\text { Statistic } \\
\text { (p-value) }\end{array}$ \\
\hline $\begin{array}{l}\text { Demographic } \\
\text { Age, } \\
\text { mean years (SD) }\end{array}$ & $49(8.0)$ & $52(7.7)$ & $\begin{array}{c}11.43^{1} \\
(0.000)^{*}\end{array}$ & $49(7.3)$ & $51(7.5)$ & $\begin{array}{c}11.20^{1} \\
(0.000)^{*}\end{array}$ \\
\hline Female, \% (n) & $\begin{array}{l}74.2 \% \\
(809)\end{array}$ & $\begin{array}{l}56.6 \% \\
(2,235)\end{array}$ & $\begin{array}{c}109.97^{2} \\
(0.000)^{*}\end{array}$ & $\begin{array}{l}79.0 \% \\
(1,203)\end{array}$ & $\begin{array}{l}60.2 \% \\
(3,335)\end{array}$ & $\begin{array}{c}184.03^{2} \\
(0.000)^{*}\end{array}$ \\
\hline $\begin{array}{r}\text { Race, } \%(n) \\
\text { White }\end{array}$ & $\begin{array}{l}95.1 \% \\
(1,051)\end{array}$ & $\begin{array}{l}96.7 \% \\
(3,810)\end{array}$ & $\begin{array}{c}6.19^{2} \\
(0.013)^{*}\end{array}$ & $\begin{array}{l}96.5 \% \\
(1,282)\end{array}$ & $\begin{array}{l}96.8 \% \\
(4,683)\end{array}$ & $\begin{array}{c}0.41^{2} \\
(0.521)\end{array}$ \\
\hline $\begin{array}{r}\text { Countysmokerate } \\
>=31 \%\end{array}$ & $\begin{array}{l}24.9 \% \\
(278)\end{array}$ & $\begin{array}{l}23.9 \% \\
(958)\end{array}$ & $\begin{array}{c}0.489 \\
(0.485)\end{array}$ & $\begin{array}{l}88.6 \% \\
(1,350)\end{array}$ & $\begin{array}{l}89.5 \% \\
(4,961)\end{array}$ & $\begin{array}{c}0.96^{2} \\
(0.327)\end{array}$ \\
\hline $\begin{array}{l}\text { Overall } \\
\text { comorbidity } \\
\text { D'Hoore-CCI }^{\mathrm{a}}\end{array}$ & & & & & & \\
\hline $\begin{array}{r}\text { Mean }(S D) \\
\text { Median }\end{array}$ & $\begin{array}{c}1.9(2.1) \\
2.0\end{array}$ & $\begin{array}{c}1.8(2.2) \\
1.0\end{array}$ & $\begin{array}{l}-1.170 \\
(0.242)\end{array}$ & $\begin{array}{c}1.8(2.0) \\
2.0\end{array}$ & $\begin{array}{c}1.6(2.1) \\
1.0\end{array}$ & $\begin{array}{c}-4.21^{3} \\
(0.000)^{*}\end{array}$ \\
\hline $\begin{array}{l}\text { Number of other } \\
\text { chronic } \\
\text { conditions } \\
\text { Mean (SD) } \\
\text { Median }\end{array}$ & $\begin{array}{c}2.4(1.4) \\
2.0\end{array}$ & $\begin{array}{c}2.1(1.4) \\
2.0\end{array}$ & $\begin{array}{c}-6.839 \\
(0.000)^{*}\end{array}$ & $\begin{array}{c}2.3(1.4) \\
2.0\end{array}$ & $\begin{array}{c}2.0(1.4) \\
2.0\end{array}$ & $\begin{array}{c}-7.2^{3} \\
(0.000)^{*}\end{array}$ \\
\hline $\begin{array}{l}\text { COPD-related } \\
\text { comorbid } \\
\text { disorders }\end{array}$ & & & & & & \\
\hline $\begin{array}{l}\text { Upper respiratory } \\
\text { tract infections }\end{array}$ & $\begin{array}{l}67.1 \% \\
(748)\end{array}$ & $\begin{array}{l}47.3 \% \\
(1,896)\end{array}$ & $\begin{array}{l}136.15^{1} \\
(0.000)^{*}\end{array}$ & $\begin{array}{l}77.8 \% \\
(1,185)\end{array}$ & $\begin{array}{l}57.8 \% \\
(3,203)\end{array}$ & $\begin{array}{l}203.30^{1} \\
(0.000)^{*}\end{array}$ \\
\hline $\begin{array}{l}\text { Lower respiratory } \\
\text { tract infections }\end{array}$ & $\begin{array}{l}27.1 \% \\
(302)\end{array}$ & $\begin{array}{l}19.5 \% \\
(780)\end{array}$ & $\begin{array}{c}30.30^{1} \\
(0.000)^{*}\end{array}$ & $\begin{array}{l}32.8 \% \\
(499)\end{array}$ & $\begin{array}{l}19.4 \% \\
(1,074)\end{array}$ & $\begin{array}{l}123.67^{1} \\
(0.000)^{*}\end{array}$ \\
\hline Septicimia & $1.3 \%(15)$ & $2.0 \%(82)$ & $\begin{array}{c}2.31^{1} \\
(0.128)\end{array}$ & $2.6 \%(39)$ & $2.1 \%(117)$ & $\begin{array}{c}1.118 \\
(0.290)\end{array}$ \\
\hline Allergic rhinitis & $19.5 \%$ & $10.0 \%$ & $73.87^{1}$ & $22.1 \%$ & $11.8 \%$ & 105.64 \\
\hline
\end{tabular}




\begin{tabular}{|c|c|c|c|c|c|c|}
\hline & (217) & (400) & $(0.000)^{*}$ & (337) & (654) & $(0.000)^{*}$ \\
\hline $\begin{array}{l}\text { Other diseases of } \\
\text { the lung }\end{array}$ & $\begin{array}{l}37.0 \% \\
(413)\end{array}$ & $\begin{array}{l}28.0 \% \\
(1,120)\end{array}$ & $\begin{array}{c}34.25^{1} \\
(0.000)^{*}\end{array}$ & $\begin{array}{c}35.8 \% \\
(545)\end{array}$ & $\begin{array}{c}27.5 \% \\
(1,523)\end{array}$ & $\begin{array}{c}39.79^{1} \\
(0.000)^{*}\end{array}$ \\
\hline $\begin{array}{r}\text { Congestive heart } \\
\text { failure }\end{array}$ & $\begin{array}{l}22.2 \% \\
(248)\end{array}$ & $\begin{array}{l}21.8 \% \\
(873)\end{array}$ & $\begin{array}{l}0.101^{1} \\
(0.751)\end{array}$ & $\begin{array}{c}24.8 \% \\
(377)\end{array}$ & $\begin{array}{l}20.7 \% \\
(1,149)\end{array}$ & $\begin{array}{c}11.41^{1} \\
(0.001)^{*}\end{array}$ \\
\hline $\begin{array}{l}\text { Disorders of fluid, } \\
\text { electrolyte, and } \\
\text { acid-base balance }\end{array}$ & $\begin{array}{c}24.0 \% \\
(268)\end{array}$ & $\begin{array}{l}19.1 \% \\
(764)\end{array}$ & $\begin{array}{c}13.33^{1} \\
(0.000)^{*}\end{array}$ & $\begin{array}{c}23.0 \% \\
(350)\end{array}$ & $\begin{array}{c}17.4 \% \\
(964)\end{array}$ & $\begin{array}{c}24.63^{1} \\
(0.000)^{*}\end{array}$ \\
\hline
\end{tabular}

$* \mathrm{p}<0.05$

${ }^{a}$ Excludes asthma and congestive heart failure

${ }^{\mathrm{b}}$ Includes hypercholesterolemia, hypertension, other heart disease, AIDS, rheumatoid arthritis, osteoarthritis, gall bladder disease, depression and anxiety, and schizophrenia.

${ }^{\mathrm{c}}$ Includes influenza, acute bronchitis and bronchiolitis, bronchitis not specified as acute or chronic, acute nasopharyngitis, acute laryngitis, acute tracheitis, acute laryngotracheitis, acute epiglottis, supraglottis, acute laryngopharyngitis, acute upper respiratory infections of multiple and unspecified sites.

${ }^{\mathrm{d}}$ Includes pneumonia from bacterial, viral, other specified organisms, and organism unspecified.

${ }^{\mathrm{e}}$ Includes pleurisy, pneumothorax, abscess of lung and mediastinum, pulmonary congestion and hypostasis, pulmonary collapse, interstitial emphysema, compensatory emphysema, pulmonary eosinophilia, acute edema of lung, pulmonary insufficiency following trauma and surgery, allergic bronchopulmonary aspegillosis, and acute and chronic respiratory failure.

${ }^{1}$ t-test statistic ${ }^{2}$ Chi-square test statistic ${ }^{3}$ Mann-Whitney U-statistic

Tables 18 and 19 compare the COPD-related utilization and costs for recipients with COPD with and without concomitant asthma in WV and KY Medicaid, respectively. A significantly higher proportion of recipients with COPD with concomitant asthma in both states had at least one COPD-related hospitalization compared to those without asthma (Table 18 _WV: $42.8 \%$ vs. $30.2 \%$; Table 19_KY: 46.5\% vs. $30.6 \%$ ) together with a higher mean number of COPD-related hospitalizations per recipient. Similar trends were seen for ER visits in both states (Table 18_WV: 42.5\% vs. 27.7\%; Table 19_KY: 40.1\% vs. 26.7\%). Similar proportions of recipients with COPD with and without asthma had at least one physician visit in WV Medicaid; however the mean number of COPD-related physician visits was significantly higher for those with asthma (Table 18_WV: 2.32 vs. 2.05). These measures were both significantly higher for KY Medicaid recipients with COPD with asthma compared to those without asthma. Recipients with COPD with concomitant asthma had a significantly higher mean number of fills in both states. On average, recipients with COPD with asthma were prescribed one additional COPD-related drug class compared to those 
without asthma in both states (Table 18_WV: 4 vs. 3; Table 19_KY: 5 vs. 4). This difference was statistically significant.

Tables 20 and 21 present the proportion of recipients with COPD using each of eight COPD-related drug classes and home oxygen therapy in WV and KY Medicaid, respectively. A higher proportion of recipients with COPD with concomitant asthma were found to be using drugs for COPD in all classes of COPD-related drugs in both states (Table 20 and Table 21). Of note, the maximum difference in proportion between the two groups was seen for the use of short-acting beta-agonists (Table 20_WV: 74.7\% vs. 54.5\%; Table 21_KY: $86.1 \%$ vs. $65.7 \%$ ) and oral corticosteroids (Table 20_WV: 63.7\% vs. 36.2\%; Table 21_KY: $71.8 \%$ vs. $43.3 \%$ ). Another interesting observation was that at least 50 percent of recipients with COPD with asthma were found to be using inhaled corticosteroids in KY Medicaid compared to 39 percent in WV Medicaid. Additionally, the difference in proportions using inhaled corticosteroids between recipients with COPD with and without asthma was comparable to that for short-acting beta-agonists and oral corticosteorids (Table 21_KY: $50.5 \%$ vs. 30.7\%). This difference in proportion was lower in WV Medicaid (Table 20_WV: $38.9 \%$ vs. $24.9 \%$ ). In WV Medicaid, similar proportions of recipients with COPD with and without concomitant asthma were found to be using home oxygen therapy. In KY Medicaid, however, a significantly higher proportion of recipients with COPD with asthma were using home oxygen therapy (Table 21_KY: 31.7\% vs. 24.6\%) compared to those without asthma.

The presence of an asthma diagnosis significantly increased the average COPD-related cost of a recipient with COPD by $\$ 2,271$ in WV Medicaid (Table 22) and by $\$ 3,518$ (Table 23 ) in KY Medicaid. For both states, univariate analyses showed statistically significant higher cost for all COPD-related cost components for recipients with COPD with asthma compared to those without asthma. In multivariate analyses, recipients with COPD with asthma had a higher cost for all COPD-related cost components. However, the cost difference per recipient was statistically significant only for prescription drugs in both states. Recipients with COPD with asthma were found to incur almost 50\% higher costs in COPDrelated prescription drug costs compared to those without asthma (Table 22_WV: 48.5\%; Table 23_KY: 50.7\%). No difference was seen in both states between recipients with COPD with and without asthma in costs for physician visits. Contrary to the results found for the WV Medicaid sample, costs for hospitalization, ER, and outpatient was found to be 
statistically significantly higher for recipients with COPD with asthma compared to those without asthma (Table 22_WV \& Table 23_KY). However, it is interesting to note that asthma significantly increased the odds of a COPD-related hospitalization in both states, as noted in part 1 of the two-part cost model (See Appendix A: Table A15_WV: OR=1.561 \& Table A21_KY: OR=1.633). A similar pattern was seen for a COPD-related ER visit (See Appendix A: Table A16_WV: OR=1.608 \& Table A22_KY: OR=1.448). 
TABLE 18: COPD-related ${ }^{\mathrm{a}}$ utilization and costs per recipient for recipients with COPD with and without concomitant asthma in WV Medicaid

\begin{tabular}{|c|c|c|c|c|}
\hline $\begin{array}{c}\text { COPD- } \\
\text { Related }^{\text {a }} \text { Cost } \\
\text { Component } \\
\text { (unit of use) }\end{array}$ & $\begin{array}{l}\text { COPD with } \\
\text { asthma } \\
(\mathrm{N}=1,115)\end{array}$ & $\begin{array}{l}\text { COPD without } \\
\text { asthma } \\
(\mathrm{N}=\mathbf{4 , 0 0 5})\end{array}$ & & $\begin{array}{c}\text { Test Statistic } \\
\text { (p-value) }\end{array}$ \\
\hline \multicolumn{5}{|c|}{$\%(n)$ with use } \\
\hline Hosp & $42.8 \%(477)$ & $30.2 \%(1,211)$ & & $62.09^{1}(0.000)^{*}$ \\
\hline ER & $42.5 \%(474)$ & $27.7 \%(1,108)$ & & $90.03^{1}(0.000)^{*}$ \\
\hline Outpatient & $91.3 \%(1,018)$ & $92.3 \%(3,695)$ & & $1.10^{1}(0.295)$ \\
\hline - Physician ${ }^{\mathrm{b}}$ & $65.6 \%{ }^{\mathrm{b}}(731)$ & $62.6 \%{ }^{\mathrm{b}}(2,508)$ & & $3.24^{1}(0.07)$ \\
\hline Prescription & $93.2 \%(1,039)$ & $88.2 \%(3,533)$ & & $22.53^{1}(0.000)^{*}$ \\
\hline \multicolumn{5}{|c|}{ Amount of use, Mean per recipient (95\% CI) } \\
\hline $\begin{array}{l}\text { Number of } \\
\text { hosp visits }\end{array}$ & $\begin{array}{c}0.80 \\
(0.72-0.89)\end{array}$ & $\begin{array}{c}0.48 \\
(0.45-0.51)\end{array}$ & & $\begin{array}{c}1923298.5^{2} \\
(0.000)^{*}\end{array}$ \\
\hline $\begin{array}{l}\text { Number of ER } \\
\text { visits }\end{array}$ & $\begin{array}{c}0.86 \\
(0.76-0.97)\end{array}$ & $\begin{array}{c}0.45 \\
(0.40-0.49)\end{array}$ & & $\begin{array}{c}1859533.5^{2} \\
(0.000)^{*}\end{array}$ \\
\hline $\begin{array}{l}\text { Number of } \\
\text { Outpatient } \\
\text { encounters }^{\mathrm{c}}\end{array}$ & $\begin{array}{c}9.14 \\
(8.53-9.75)\end{array}$ & $\begin{array}{c}8.35 \\
(8.04-8.66)\end{array}$ & & $\begin{array}{l}2148398.0^{2} \\
\quad(0.052)\end{array}$ \\
\hline $\begin{array}{l}\text { - Number of } \\
\text { physician } \\
\text { visits }^{b}\end{array}$ & $\begin{array}{c}2.32^{\mathrm{b}} \\
(2.15-2.49)\end{array}$ & $\begin{array}{c}2.05^{\mathrm{b}} \\
(1.97-2.13)\end{array}$ & & $\begin{array}{c}2120443.0^{2} \\
(0.008)^{*}\end{array}$ \\
\hline $\begin{array}{l}\text { Number of } \\
\text { prescription } \\
\text { fills }\end{array}$ & $\begin{array}{c}21.33 \\
(20.31-22.36)\end{array}$ & $\begin{array}{c}14.72 \\
(14.23-15.21)\end{array}$ & & $\begin{array}{c}1626838.0^{2} \\
(0.000)^{*}\end{array}$ \\
\hline $\begin{array}{l}\text { - Number of } \\
\text { unique COPD- } \\
\text { related drug } \\
\text { classes }\end{array}$ & $\begin{array}{c}4.04 \\
(3.93-4.14)\end{array}$ & $\begin{array}{c}2.90 \\
(2.85-2.96)\end{array}$ & & $\begin{array}{l}-18.07^{3} \\
(0.000)^{*}\end{array}$ \\
\hline \multicolumn{5}{|c|}{ Costs, Mean per recipient (95\% CI) } \\
\hline Hosp & $\begin{array}{c}\$ 4,041 \\
(\$ 3,492-\$ 4,590)\end{array}$ & $\begin{array}{c}\$ 2,531 \\
(\$ 2,321-\$ 2,741)\end{array}$ & $\begin{array}{l}\text { Difference } \\
\$ 1,510\end{array}$ & $\begin{array}{c}1941964.5^{2} \\
(0.000)^{*}\end{array}$ \\
\hline
\end{tabular}




\begin{tabular}{lcccc}
\hline ER visit & $\begin{array}{l}\$ 365 \\
(\$ 314-\$ 417)\end{array}$ & $\begin{array}{c}\$ 190 \\
(\$ 169-\$ 211)\end{array}$ & $\$ 175$ & $\begin{array}{c}1875579.5^{2} \\
(0.000)^{*}\end{array}$ \\
Outpatient & $\$ 1,114$ & $\$ 984$ & $\$ 130$ & $2095245.5^{2}$ \\
& $(\$ 1,012-\$ 1,216)$ & $(\$ 929-\$ 1,039)$ & & $(0.000)^{*}$ \\
- Physician & $\$ 187^{\mathrm{b}}$ & $\$ 146^{\mathrm{b}}$ & $\$ 41^{\mathrm{b}}$ & $2045981.5^{2}$ \\
visit & $(\$ 161-\$ 212)$ & $(\$ 134-\$ 157)$ & & $(0.000)^{*}$ \\
$\begin{array}{l}\text { Prescription } \\
\text { drugs }\end{array}$ & $\$ 1,227$ & $\$ 807$ & $\$ 420$ & $1635821.5^{2}$ \\
& $(\$ 1,154-\$ 1,299)$ & $(\$ 776-\$ 839)$ & & $(0.000)^{*}$ \\
Total costs & $\$ 6,747$ & $\$ 4,513$ & $\$ 2,234$ & $1725798.0^{2}$ \\
& $(\$ 6,122-\$ 7,372)$ & $(\$ 4,265-\$ 4,761)$ & & $(0.000)^{*}$ \\
\hline
\end{tabular}

$* \mathrm{p}<0.05$

${ }^{a}$ COPD-related costs were computed from the paid amounts of the medical claims with a primary or secondary diagnosis of COPD, and prescription claims of COPD-related drug classes. COPD-related drug classes include: anticholinergics(ipratropium, ipratropium/albuterol), inhaled long-acting beta-agonists, short-acting beta-agonists, inhaled corticosteroids, inhaled corticosteroid/long-acting beta-agonist combination, methylxanthines, oral corticosteroids, and antibiotics for respiratory infections. COPD-related nursing home costs could not be calculated due to the absence of diagnoses codes on nursing home claims.

${ }^{\mathrm{b}}$ Physician cost component is a sub-component of the Outpatient cost component.

${ }^{\mathrm{c}}$ An outpatient encounter is defined as any service for a particular recipient on a unique date that generated a CPT code other than 99281-99285, 99288.

${ }^{1}$ Chi-square test statistic; ${ }^{2}$ Mann-Whitney U-statistic; ${ }^{3} \mathrm{t}$ statistic 
TABLE 19: COPD-related ${ }^{\mathrm{a}}$ utilization and costs per recipient for recipients with COPD with and without concomitant asthma in KY Medicaid

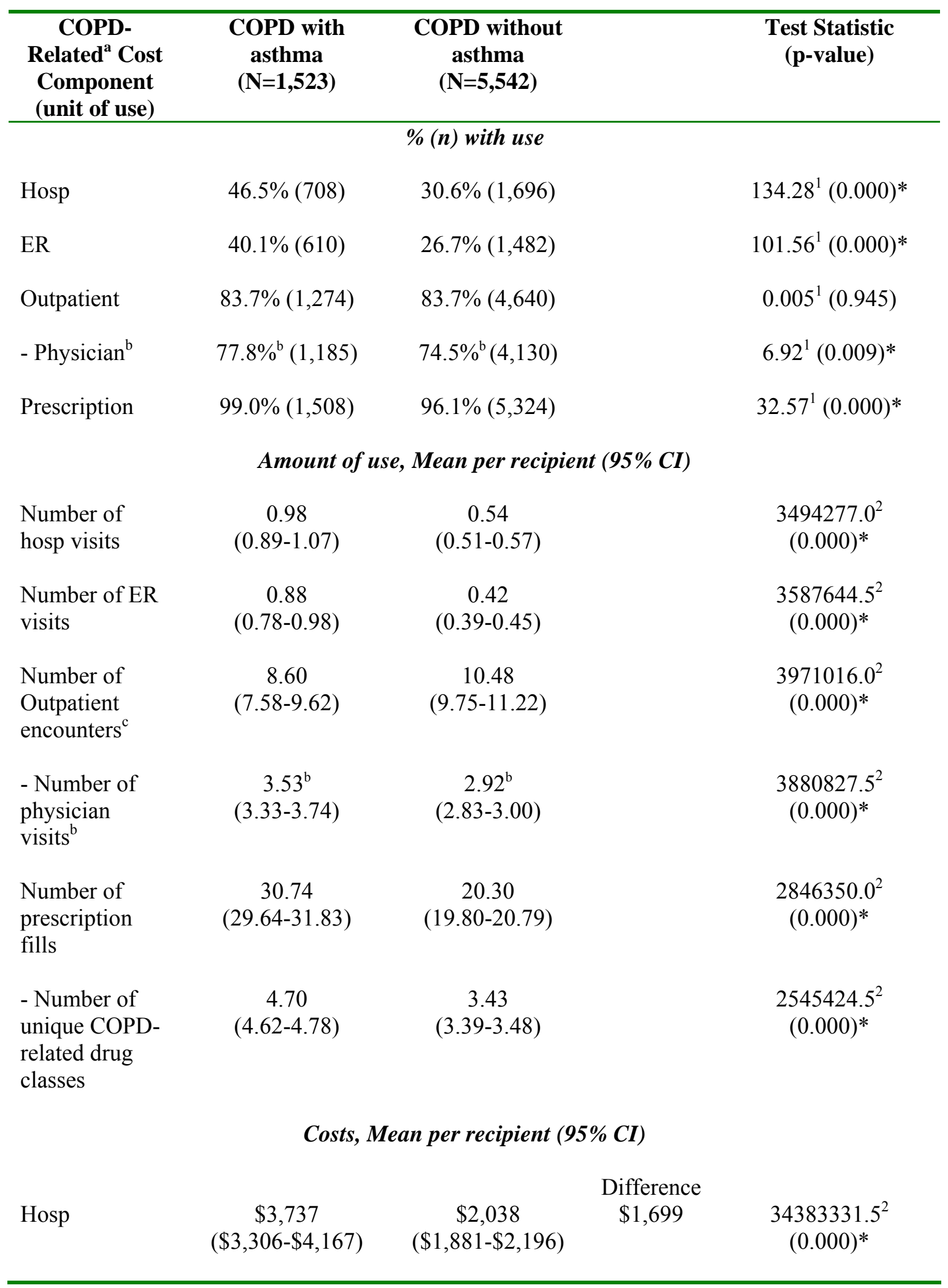




\begin{tabular}{lcccc}
\hline ER visit & $\$ 402$ & $\$ 212$ & $\$ 190$ & $3603196.5^{2}$ \\
& $(\$ 349-\$ 454)$ & $(\$ 193-\$ 231)$ & & $(0.000)^{*}$ \\
Outpatient & $\$ 2,653$ & $\$ 2,289$ & $\$ 364$ & $3870278.0^{2}$ \\
& $(\$ 2,411-\$ 2,896)$ & $(\$ 2,178-\$ 2,400)$ & & $(0.000)^{*}$ \\
- Physician & $\$ 343^{\mathrm{b}}$ & $\$ 271^{\mathrm{b}}$ & $\$ 72^{\mathrm{b}}$ & $3896708.0^{2}$ \\
visit $^{\mathrm{b}}$ & $(\$ 313-\$ 374)$ & $(\$ 259-\$ 284)$ & & $(0.000)^{*}$ \\
$\begin{array}{l}\text { Prescription } \\
\text { drugs }\end{array}$ & $\$ 1,826$ & $\$ 1,182$ & $\$ 644$ & $2875742.0^{2}$ \\
& $(\$ 1,749-\$ 1,902)$ & $(\$ 1,148-\$ 1,217)$ & & $(0.000)^{*}$ \\
Total costs & $\$ 8,617$ & $\$ 5,721$ & $\$ 2,896$ & $3151009.5^{2}$ \\
& $(\$ 7,982-\$ 9,253)$ & $(\$ 5,480-\$ 5,963)$ & & $(0.000)^{*}$ \\
\hline
\end{tabular}

$* \mathrm{p}<0.05$

${ }^{a}$ COPD-related costs were computed from the paid amounts of the medical claims with a primary or secondary diagnosis of COPD, and prescription claims of COPD-related drug classes. COPD-related drug classes include: anticholinergics(ipratropium, ipratropium/albuterol), inhaled long-acting beta-agonists, short-acting beta-agonists, inhaled corticosteroids, inhaled corticosteroid/long-acting beta-agonist combination, methylxanthines, oral corticosteroids, and antibiotics for respiratory infections. COPD-related nursing home costs could not be calculated due to the absence of diagnoses codes on nursing home claims.

${ }^{\mathrm{b}}$ Physician cost component is a sub-component of the Outpatient cost component.

${ }^{\mathrm{c}}$ An outpatient encounter is defined as any service for a particular recipient on a unique date that generated a CPT code other than 99281-99285, 99288.

${ }^{1}$ Chi-square test statistic; ${ }^{2}$ Mann-Whitney U-statistic; ${ }^{3} \mathrm{t}$ statistic 
TABLE 20: Utilization of COPD-related prescription drugs and home oxygen therapy for recipients with COPD with and without concomitant asthma in WV Medicaid

\begin{tabular}{|c|c|c|c|c|}
\hline \multicolumn{2}{|r|}{ Prescription drug category } & \multirow{2}{*}{ 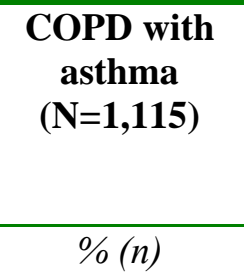 } & \multirow{2}{*}{$\begin{array}{c}\begin{array}{c}\text { COPD } \\
\text { without } \\
\text { asthma } \\
(\mathbf{N}=\mathbf{4 , 0 0 5 )}\end{array} \\
\%(n)\end{array}$} & \multirow{2}{*}{$\begin{array}{c}\text { Test Statistic } \\
\text { (p-value) }\end{array}$} \\
\hline & & & & \\
\hline 1. C & COPD-related prescription drug use & $93.2 \%(1,039)$ & $88.2 \%(3,533)$ & $\begin{array}{c}22.53^{1} \\
(0.000)^{*}\end{array}$ \\
\hline & $\begin{array}{l}\text { anticholinergics (Ipratropium, } \\
\text { Ipratropium/Albuterol combination) }\end{array}$ & $53.1 \%(592)$ & $41.0 \%(1,641)$ & $\begin{array}{c}52.10^{1} \\
(0.000)^{*}\end{array}$ \\
\hline & b. Long-acting beta-agonists & $22.6 \%(252)$ & $14.8 \%(593)$ & $\begin{array}{c}38.45^{1} \\
(0.000)^{*}\end{array}$ \\
\hline & c. Short-acting beta-agonists & $74.7 \%(833)$ & $54.5 \%(2,184)$ & $\begin{array}{c}146.70^{1} \\
(0.000)^{*}\end{array}$ \\
\hline & d. Inhaled corticosteroids & $38.9 \%(434)$ & $24.9 \%(996)$ & $\begin{array}{c}85.59^{1} \\
(0.000)^{*}\end{array}$ \\
\hline & $\begin{array}{l}\text { Inhaled corticosteroids/Long-acting } \\
\text { beta-agonist combination } \\
\text { (Fluticasone/Salmeterol) }\end{array}$ & $36.9 \%(411)$ & $22.5 \%(901)$ & $\begin{array}{c}94.42^{1} \\
(0.000)^{*}\end{array}$ \\
\hline & f. Methylxanthines & $27.4 \%(305)$ & $19.5 \%(779)$ & $\begin{array}{c}32.65^{1} \\
(0.000)^{*}\end{array}$ \\
\hline & g. Oral corticosteroids & $63.7 \%(710)$ & $36.2 \%(1,450)$ & $\begin{array}{l}269.90^{1} \\
(0.000)^{*}\end{array}$ \\
\hline $\mathrm{h}$ & $\begin{array}{l}\text { h. Antibiotics for respiratory } \\
\text { infections }\end{array}$ & $86.4 \%(963)$ & $77.0 \%(3,084)$ & $\begin{array}{c}46.17^{1} \\
(0.000)^{*}\end{array}$ \\
\hline 2. L & Use of home oxygen therapy & $31.5 \%(351)$ & $29.5 \%(1,181)$ & $\begin{array}{l}1.65^{1} \\
(0.199)\end{array}$ \\
\hline
\end{tabular}

$* \mathrm{p}<0.05$

${ }^{1}$ Chi-square test statistic 
TABLE 21: Utilization of COPD-related prescription drugs and home oxygen therapy for recipients with COPD with and without concomitant asthma in KY Medicaid

\begin{tabular}{|c|c|c|c|}
\hline Prescription drug category & $\begin{array}{l}\text { COPD with } \\
\text { asthma } \\
(\mathrm{N}=1,523)\end{array}$ & $\begin{array}{l}\text { COPD without } \\
\text { asthma } \\
(\mathrm{N}=5,542)\end{array}$ & $\begin{array}{l}\text { Test } \\
\text { Statistic (p- } \\
\text { value) }\end{array}$ \\
\hline & $\%(n)$ & $\%(n)$ & \\
\hline 1. COPD-related prescription drug use & $100.0 \%(1,523)$ & $96.6 \%(4,894)$ & $\begin{array}{l}3.62^{1} \\
(0.06)\end{array}$ \\
\hline $\begin{array}{l}\text { a. Anticholinergics (Ipratropium, } \\
\text { Ipratropium/Albuterol combination) }\end{array}$ & $62.3 \%(949)$ & $51.0 \%(2,826)$ & $\begin{array}{c}61.51^{1} \\
(0.000)^{*}\end{array}$ \\
\hline b. Long-acting beta-agonists & $30.5 \%(464)$ & $19.7 \%(1,090)$ & $\begin{array}{c}81.19^{1} \\
(0.000)^{*}\end{array}$ \\
\hline c. Short-acting beta-agonists & $86.1 \%(1,312)$ & $65.7 \%(3,640)$ & $\begin{array}{c}238.7^{1} \\
(0.000)^{*}\end{array}$ \\
\hline d. Inhaled corticosteroids & $50.5 \%(769)$ & $30.7 \%(1,704)$ & $\begin{array}{l}204.7^{1} \\
(0.000)^{*}\end{array}$ \\
\hline $\begin{array}{l}\text { e. Inhaled corticosteroids/Long-acting } \\
\text { beta-agonist combination } \\
\text { (Fluticasone/Salmeterol) }\end{array}$ & $38.1 \%(581)$ & $21.0 \%(1,163)$ & $\begin{array}{c}189.2^{1} \\
(0.000)^{*}\end{array}$ \\
\hline f. Methylxanthines & $35.9 \%(547)$ & $24.7 \%(1,369)$ & $\begin{array}{c}76.01^{1} \\
(0.000)^{*}\end{array}$ \\
\hline g. Oral corticosteroids & $71.8 \%(1,094)$ & $43.3 \%(2,400)$ & $\begin{array}{l}388.9^{1} \\
(0.000)^{*}\end{array}$ \\
\hline h. Antibiotics for respiratory infections & $94.6 \%(1,440)$ & $86.9 \%(4,818)$ & $\begin{array}{c}68.46^{1} \\
(0.000)^{*}\end{array}$ \\
\hline 2. Use of home oxygen therapy & $31.7 \%(483)$ & $24.6 \%(1,365)$ & $\begin{array}{c}34.04^{1} \\
(0.000)^{*}\end{array}$ \\
\hline
\end{tabular}

$* \mathrm{p}<0.05$

${ }^{1}$ Chi-square test statistic 
TABLE 22: Mean incremental COPD-related cost per recipient for recipients with COPD with and without concomitant asthma in WV Medicaid

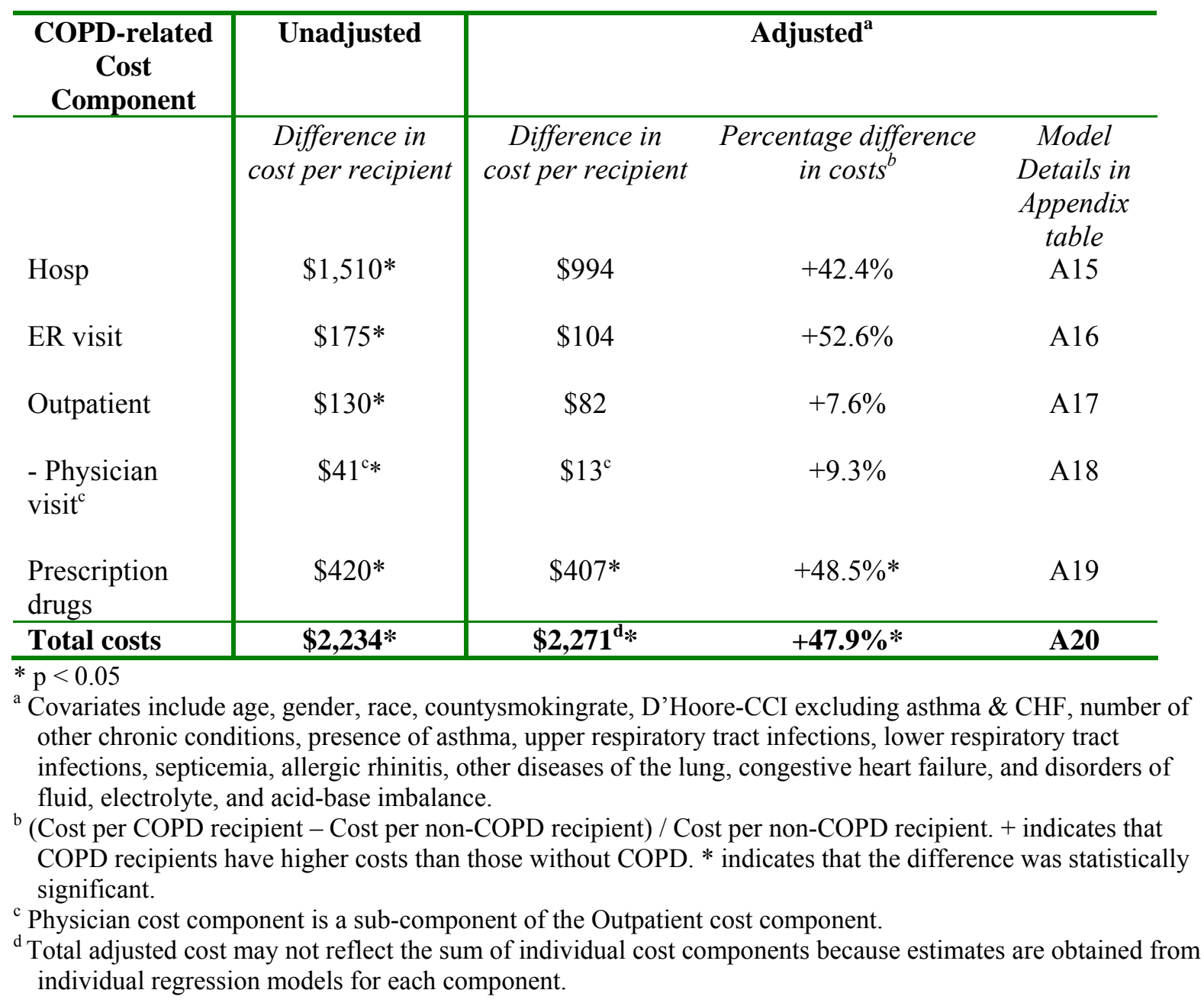


TABLE 23: Mean incremental COPD-related costs per recipient for recipients with COPD with and without concomitant asthma in KY Medicaid

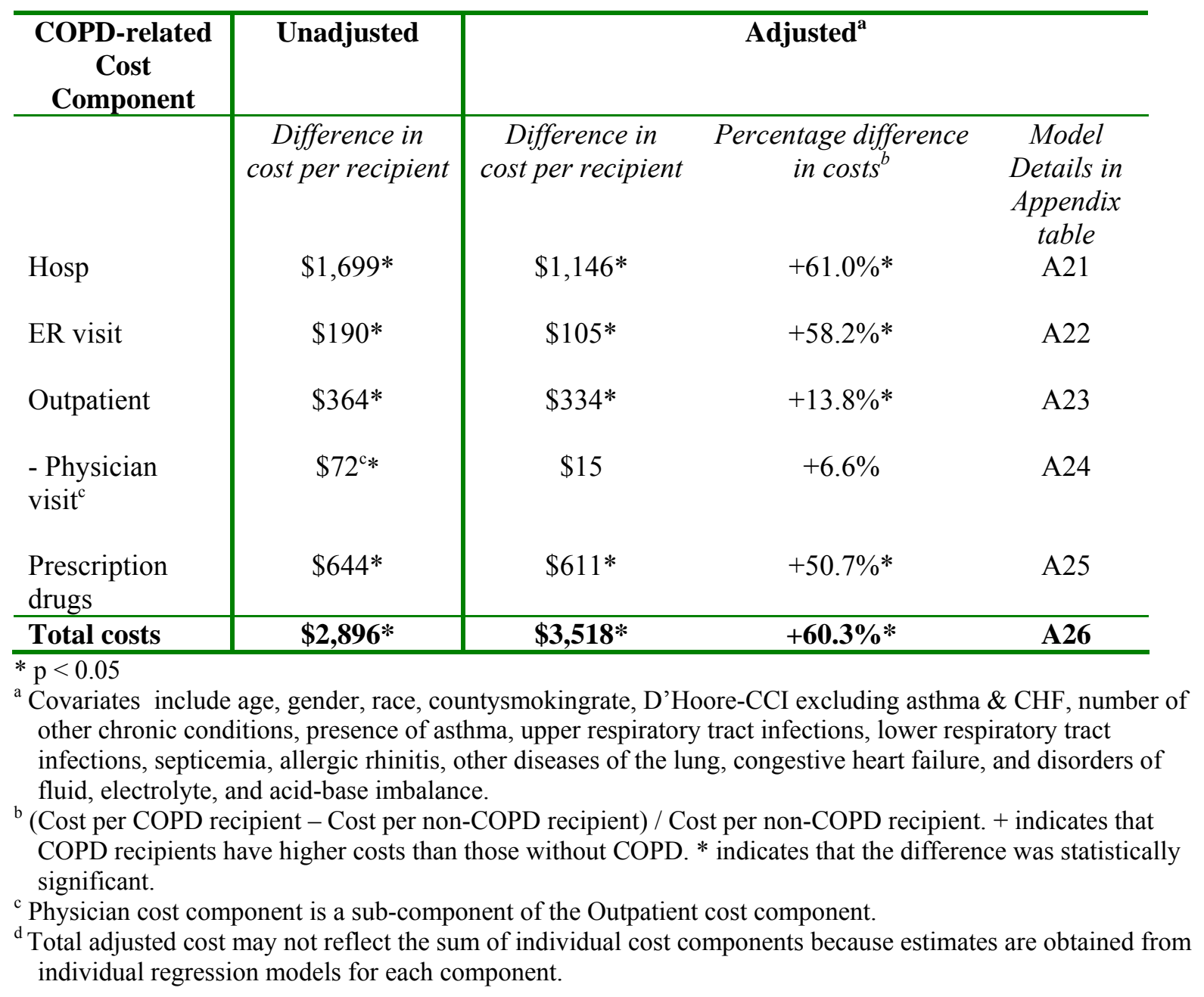




\section{Discussion for Research Objective 2}

The airflow limitation in COPD was initially thought to be completely irreversible while that in asthma was thought to be completely reversible. However, studies conducted in the past few years that have shown the presence of significant reversibility or partial reversibility in patients with COPD, and of non-reversible airflow obstruction in patients with asthma demonstrate that these conditions can coexist (Mannino, 2002). In our study, 22 percent of the total recipients identified as having COPD also had a concomitant diagnosis of asthma similar to the 21 percent found in the 1996 National Health Interview Survey (NHIS) (Mannino, 2002). A higher proportion was found in another study that analyzed data from medical records of patients with COPD with almost half of all patients with COPD having asthma (Mapel, 2004).

There are no studies in the literature that have determined if the presence of asthma increases the economic burden for patients with COPD although some preliminary comparisons from the 1996 NHIS data provides some indication (Mannino, 2002). In that study, the lung function, respiratory symptoms, and activity limitation was compared among patients with COPD with asthma versus those without asthma. A total of 29.9 percent reportedly had low lung function compared to 17.4 percent of patients with COPD without asthma. Additionally, differences were seen in almost all characteristic COPD symptoms of cough, phlegm, wheeze, and shortness of breath with at least 95 percent versus 84 percent experiencing any symptom for those with and without asthma, respectively. The significantly increased prevalence of other respiratory conditions found in our study for recipients with COPD with concomitant asthma may explain the higher proportions experiencing symptoms in the NHIS. The NHIS only documented indicators of indirect costs between COPD patients with and without asthma, and found significantly more days of restricted activity, bed confinement, and work loss.

Our study documented only direct medical costs and showed that recipients with COPD with asthma have increased use of COPD-related healthcare resources than those without asthma. The marginal cost of asthma was quite substantial, and ranged from $\$ 2,200$ in WV Medicaid to $\$ 3,500$ in KY Medicaid, reflecting a percentage difference of 50 to 60 percent. Of note, asthma significantly increased the odds of having a COPD-related hospitalization and ER visit for recipients with COPD in both states. Although the cost differences for 
hospitalization, ER, and outpatient cost components were found to be higher for those with a concomitant diagnosis of asthma in both states, this difference was statistically significant only in the KY Medicaid sample. This discrepancy may point to a difference in treatment approaches of recipients with COPD with asthma versus without asthma in the two states. An indication of this differential approach can be seen in the use of inhaled corticosteroids and home oxygen therapy in the two states. Specifically, in KY Medicaid, at least 50 percent of the recipients with COPD with asthma were found to be using inhaled corticosteroids compared to 39 percent in WV Medicaid. Additionally, the difference in proportions using inhaled corticosteroids between recipients with COPD with and without asthma in KY Medicaid (Table 21_KY: $50.5 \%$ vs. $30.7 \%$ ) was lower to the difference in proportion in WV Medicaid (Table 20_WV: $38.9 \%$ vs. $24.9 \%$ ). Also, the proportion using home oxygen therapy was significantly higher for the recipients with COPD with concomitant asthma in the KY Medicaid sample contrary to the WV Medicaid sample where similar proportions were found. Thus, it is probable that recipients with COPD in KY Medicaid with concomitant asthma are treated using more resources compared to those in WV Medicaid, and may explain the statistically significant higher COPD-related cost components found in the former sample.

Another interesting finding was that after controlling for demographic characteristics and comorbid conditions, the presence of asthma significantly increased the costs of prescription drugs by 50 percent. It should be noted that a higher proportion of recipients with COPD with asthma were using all COPD-related drug classes, and not solely those that are more commonly used for asthma (e.g. an increase was seen for both ipratropium (primarily indicated for COPD) and inhaled corticosteroid (primarily indicated for asthma). This may indicate that the drug treatment is not specifically targeted to treating asthma but treating the respiratory syndrome as a whole. 


\section{Phase II \\ Part 1}

Phase II of the study compares costs and exacerbations of recipients initiating drug therapy. Part 1 of phase II compares these outcomes between recipients initiating therapy with a combination product of ipratropium and albuterol (IPR/ALB) or monotherapy with inhaled long-acting beta-agonist (LABA). Table 24 outlines the extraction process from raw data to obtain the study sample.

TABLE 24: Sample extraction for Phase II - Part 1

\begin{tabular}{llll}
\hline \multicolumn{1}{c}{ Step } & \multicolumn{1}{c}{ Outcome WV } & \multicolumn{1}{c}{ Outcome KY } \\
\hline 1. & & \\
\# with a Rx claim for IPR/ALB or LABA & $\mathrm{A}=19,257$ & $\mathrm{~A}=43,256$ \\
and KY WV-Jan 1, Jul 1, 1998 to Jun 30, 2002 & -IPR/ALB $=8,204$ & -IPR/ALB $=21,265$ \\
& -LABA 31,2002 & -LABA $=21,991$
\end{tabular}

2. The date of first chronologically occurring prescription claim was defined as an index date for each person

3. \# of A between 35-64 years, continuously eligible for Medicaid and not enrolled in

$$
\begin{array}{ll}
\mathrm{B}=6,646 & \mathrm{~B}=14,533 \\
-\mathrm{IPR} / \mathrm{ALB}=3,012 & -\mathrm{IPR} / \mathrm{ALB}=7,727 \\
-\mathrm{LABA}=3,634 & -\mathrm{LABA}=6,806
\end{array}
$$
managed care at anytime during study period

4. \# of B without a Rx claim for IPR/ALB or IPR, LABA, or ICS in the pre-index period and those without a Rx claim for LABA

$\mathrm{C}=2,505$ -IPR/ALB $=1,696$ $-\mathrm{LABA}=809$ $\mathrm{C}=5,364$ -IPR/ALB $=4,136$ $-\mathrm{LABA}=1,228$ (IPR/ALB group), IPR/ALB or IPR (LABA group) or ICS in the 30 days post-index period

5. \# of $\mathrm{C}$ without Home $\mathrm{O}_{2}$ therapy in preindex period

$\mathrm{D}=2,401$
$-\mathrm{IPR} / \mathrm{ALB}=1,618$
$-\mathrm{LABA}=783$

$\mathrm{E}=2,345$

-IPR/ALB $=1,581$

$-\mathrm{LABA}=764$

$\mathrm{F}=374$

-IPR/ALB $=250$

$-\mathrm{LABA}=124$
$\mathrm{D}=5,230$

$-\mathrm{IPR} / \mathrm{ALB}=4,030$

$-\mathrm{LABA}=1,200$

$\mathrm{E}=5,132$

$-\mathrm{IPR} / \mathrm{ALB}=3,952$

$-\mathrm{LABA}=1,180$

$\mathrm{F}=780$

$-\mathrm{IPR} / \mathrm{ALB}=624$

$-\mathrm{LABA}=156$

COPD but do not have a hospitalization with a primary or secondary diagnosis of COPD in pre-index period 


$$
\begin{array}{lll}
\text { 8. \# of } F \text { with at least } 1 \text { additional Rx claim of } \\
\begin{array}{ll}
\mathrm{index} \text { drug therapy in follow-up period. } \\
\end{array} & \begin{array}{l}
\mathrm{G}=213 \\
\text {-IPR/ALB }=130
\end{array} & \begin{array}{l}
\mathrm{G}=478 \\
\text {-IPR/ALB }=376 \\
\text {-LABA }=83
\end{array} \\
& \begin{array}{l}
\text { Total }=691 \\
\text { F. Final study sample size }
\end{array} \\
& \begin{array}{l}
\text {-IPR/ALB }=506 \\
\text {-LABA }=185
\end{array}
\end{array}
$$

A total of 691 recipients met the inclusion criteria for the study design of Part 1 of Phase II. A majority of the recipients were initiated on combination ipratropium/albuterol (73.2\%). The majority of the long-acting beta-agonist group was initiated on salmeterol, and only 11 people had formoterol as their index LABA as formoterol was introduced in 2002. The study design selected recipients with relatively less severe COPD by excluding those with a hospitalization for COPD and home oxygen therapy in the pre-index period. The design ensured almost comparable drug therapy groups at baseline. As can be seen in table 25, which outlines the baseline characteristics of the study sample, the groups were similar in terms of demographic characteristics except in the smoking prevalence rate of their residential county. A significantly higher proportion of recipients initiated on combination ipratropium/albuterol resided in counties with a smoking prevalence rate of at least $31 \%$. No differences were observed in overall comorbidity burden. The groups differed on two measures of COPD severity: the use and number of canisters of inhaled short-acting betaagonists (SABA), and in the use and number of prescriptions of nebulized short-acting bronchodilators (SABD). In particular, the LABA group was found to have a significantly higher proportion of recipients using inhaled SABA and nebulized SABD. No differences were seen for other measures. 
TABLE 25: Baseline characteristics of study sample by drug therapy status (IPR/ALB vs. LABA: Phase II - Part 1)

\begin{tabular}{|c|c|c|c|}
\hline Characteristic & $\begin{array}{c}\text { IPR/ALB } \\
(N=506)\end{array}$ & $\begin{array}{c}\text { LABA } \\
(\mathrm{N}=185)\end{array}$ & $\begin{array}{c}\text { Test Statistic } \\
\text { (p-value) } \\
*=\text { values }<\mathbf{0 . 0 5} \\
\end{array}$ \\
\hline \multicolumn{4}{|l|}{ Demographic } \\
\hline Age, mean years (SD) & $52(7.5)$ & $51(7.6)$ & $1.593^{1}(0.112)$ \\
\hline Female, $\%(n)$ & $56.8 \%(287)$ & $55.4 \%(102)$ & $0.107^{2}(0.744)$ \\
\hline White, \% (n) & $96.5 \%(440)$ & $97.7 \%(171)$ & $0.616^{2}(0.432)$ \\
\hline \multicolumn{4}{|l|}{ State, $\%(n)$} \\
\hline$W V$ & $25.7 \%(130)$ & $44.9 \%(83)$ & $23.355^{2}(0.000)^{*}$ \\
\hline$K Y$ & $74.3 \%(376)$ & $55.1 \%(102)$ & \\
\hline Countysmokerate $>=31 \%$ & $72.3 \%(366)$ & $63.2 \%(117)$ & $5.319^{2}(0.021)^{*}$ \\
\hline \multicolumn{4}{|l|}{ Overall Comorbidity } \\
\hline \multicolumn{4}{|l|}{ D'Hoore-CCI ${ }^{\mathrm{a}}$} \\
\hline $\begin{array}{r}\text { Mean (SD) } \\
\text { Median }\end{array}$ & $\begin{array}{c}1.2(1.7) \\
0.0\end{array}$ & $\begin{array}{c}1.1(1.8) \\
0.0\end{array}$ & $1.257^{3}(0.209)$ \\
\hline $\begin{array}{r}\text { Number of other chronic conditions }{ }^{\mathrm{b}} \\
\text { Mean (SD) } \\
\text { Median }\end{array}$ & $\begin{array}{c}1.8(1.3) \\
2.0\end{array}$ & $\begin{array}{c}1.9(1.4) \\
2.0\end{array}$ & $-0.580^{3}(0.562)$ \\
\hline \multicolumn{4}{|l|}{ COPD severity } \\
\hline $\begin{array}{r}\text { Use of inhaled SABA }{ }^{\mathrm{c}} \text { canisters, } \%(\mathrm{n}) \\
\text { Mean \# of canisters (SD) } \\
\text { Median }\end{array}$ & $\begin{array}{c}51.4 \%(260) \\
2.9(5.1)\end{array}$ & $\begin{array}{c}63.2 \%(117) \\
4.4(6.5)\end{array}$ & $\begin{array}{c}7.686^{2}(0.006)^{*} \\
-3.330^{3}(0.001)^{*}\end{array}$ \\
\hline $\begin{array}{r}\text { Use of other doseform SABD }{ }^{\mathrm{d}}, \%(\mathrm{n}) \\
\text { Mean \# of Rx (SD) } \\
\text { Median }\end{array}$ & $\begin{array}{c}25.3 \%(128) \\
1.3(3.2)\end{array}$ & $\begin{array}{c}28.7 \%(53) \\
1.7(4.0)\end{array}$ & $\begin{array}{c}0.787^{2}(0.375) \\
-0.922^{3}(0.357)\end{array}$ \\
\hline $\begin{array}{l}\text { Use of } \mathrm{OCS}^{\mathrm{e}}, \%(\mathrm{n}) \\
\text { Mean \# of Rx (SD) } \\
\text { Median }\end{array}$ & $\begin{array}{c}29.1 \%(147) \\
0.6(1.6)\end{array}$ & $\begin{array}{c}23.8 \%(44) \\
0.6(1.7)\end{array}$ & $\begin{array}{l}1.879^{2}(0.170) \\
1.347^{3}(0.178)\end{array}$ \\
\hline $\begin{array}{r}\text { Use of nebulized SABD }{ }^{\mathrm{d}}, \%(\mathrm{n}) \\
\text { Mean \# of Rx (SD) } \\
\text { Median }\end{array}$ & $\begin{array}{c}10.1 \%(51) \\
0.3(1.4) \\
0.0\end{array}$ & $\begin{array}{l}18.9 \%(35) \\
0.4(1.4) \\
0.0\end{array}$ & $\begin{array}{c}9.715^{2}(0.002)^{*} \\
-2.923^{3}(0.004)^{*}\end{array}$ \\
\hline Use of Hosp/ER for COPD-related & $43.7 \%(221)$ & $42.2 \%(78)$ & $0.127^{2}(0.722)$ \\
\hline
\end{tabular}


Research Objective 3: To compare the risk and frequency of exacerbations between recipients with COPD who initiated therapy with ipratropium/albuterol or monotherapy with inhaled long-acting beta-agonist.

A total of $141(20.4 \%)$ recipients experienced at least one exacerbation in the follow-up period, with a majority of the exacerbations being moderate in nature. The chi-square test and the log-rank test showed no differences between the drug therapy groups in the proportion experiencing an exacerbation or in the time to exacerbation, respectively, as seen in table 26. Similar results were seen in the multivariate analyses. Following no violation of the proportional hazards assumption, the Cox-proportional hazards regression model was used to determine differences between drug therapy groups in their time to exacerbation (Table 27). Similar to findings from univariate analyses, those initiated on a LABA had a lower hazard of experiencing an exacerbation compared to the IPR/ALB group, but this difference was not statistically significant (Figure 18 and Table 27). A logistic regression analyses was also conducted that determined differences in the probability of experiencing an exacerbation, and similar results were seen (Table 28). The zero-inflated Poisson model showed no differences in the frequency of exacerbations between drug therapy groups (Table 29)

Research Objective 4: To compare COPD-related costs between recipients with COPD who initiated therapy with ipratropium/albuterol or monotherapy with inhaled long-acting betaagonist.

Table 26 also lists the total COPD-related costs incurred by recipients in the two drug therapy groups. No differences were observed in total COPD-related costs, as well as in individual COPD-related cost components except for COPD-related prescription drug costs. In univariate analyses, recipients initiated on a long-acting beta-agonist were found to incur $\$ 140$ in incremental COPD-related prescription drug costs compared to those initiated on combination IPR/ALB. Multivariate semi-log OLS models were run to determine differences in total COPD-related costs, and no differences were seen (Table 30). Following the significant difference found between drug therapy groups for COPD-related prescription drug costs in univariate analyses, multivariate analyses were done only for the COPD-related prescription drug cost component, and again recipients initiated on LABA therapy were 
found to incur 23.7 percent higher costs with an adjusted incremental cost of \$163 (Table $31)$.

TABLE 26: Exacerbations and costs in follow-up period by drug therapy status (IPR/ALB vs. LABA: Phase II - Part 1)

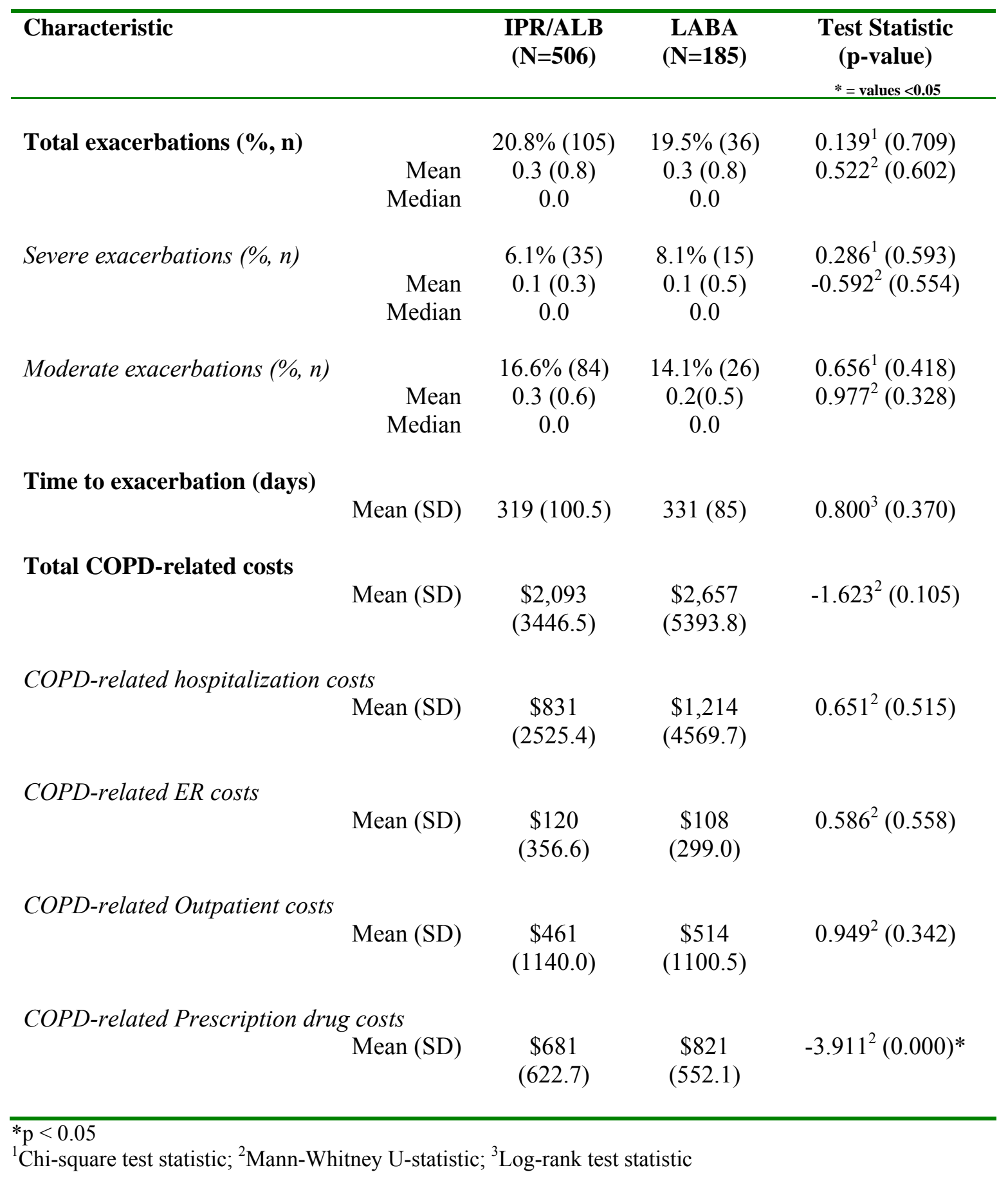


TABLE 27: Cox-Proportional hazards regression of the impact of drug therapy (IPR/ALB or LABA) on time to exacerbation

\begin{tabular}{|c|c|c|c|}
\hline \multirow[b]{2}{*}{ Variable } & \multicolumn{3}{|c|}{ DV: Time to exacerbation } \\
\hline & Hazard ratio (SE) & $95 \% C I$ & $\begin{array}{c}p \\
\text { value }\end{array}$ \\
\hline \multicolumn{4}{|l|}{ Drug therapy (Ref: IPR/ALB) } \\
\hline LABA & $0.871(0.212)$ & $0.541-1.403$ & 0.570 \\
\hline \multicolumn{4}{|l|}{ Demographic } \\
\hline Age (years) & $1.015(0.014)$ & $0.987-1.044$ & 0.281 \\
\hline Gender (Ref: Male) & & & \\
\hline Female & $1.771(0.393)$ & $1.146-2.738$ & $0.010 *$ \\
\hline Race (Ref: non-White) & & & \\
\hline White & $1.225(0.883)$ & $0.298-5.034$ & 0.779 \\
\hline State (Ref: WV ) & & & \\
\hline $\mathrm{KY}$ & $1.166(0.311)$ & $0.692-1.965$ & 0.564 \\
\hline County_Smokerate (Ref: $<31 \%$ ) & & & \\
\hline$>=31 \%$ & $1.324(0.357)$ & $0.781-2.245$ & 0.297 \\
\hline \multicolumn{4}{|l|}{ Overall comorbidity } \\
\hline D’Hoore-CCI & $0.931(0.066)$ & $0.810-1.070$ & 0.313 \\
\hline Number of other chronic conditions & $0.788(0.071)$ & $0.660-0.941$ & $0.008^{*}$ \\
\hline \multicolumn{4}{|l|}{ COPD severity } \\
\hline$\#$ of inhaled SABA canisters & $1.017(0.020)$ & $0.979-1.057$ & 0.389 \\
\hline \# of other doseform SABD prescriptions & $0.993(0.032)$ & $0.931-1.059$ & 0.826 \\
\hline \# of OCS prescriptions & $1.071(0.048)$ & $0.981-1.059$ & 0.127 \\
\hline Use of nebulized SABD & $1.140(0.333)$ & $0.643-2.021$ & 0.653 \\
\hline $\begin{array}{r}\text { \# of Hosp/ER visits for COPD-related } \\
\text { comorbid disorders }\end{array}$ & $1.373(0.091)$ & $1.205-1.564$ & $0.000^{*}$ \\
\hline $\begin{array}{r}\text { \# of Physician visits for COPD-related } \\
\text { comorbid disorders }\end{array}$ & $1.016(0.035)$ & $0.951-1.086$ & 0.632 \\
\hline Presence of asthma & $1.218(0.393)$ & $0.646-2.294$ & 0.542 \\
\hline
\end{tabular}


FIGURE 18: Adjusted Kaplan-Meier survival curve of time to exacerbation by drug therapy group (IPR/ALB vs. LABA: Phase II - Part 1)

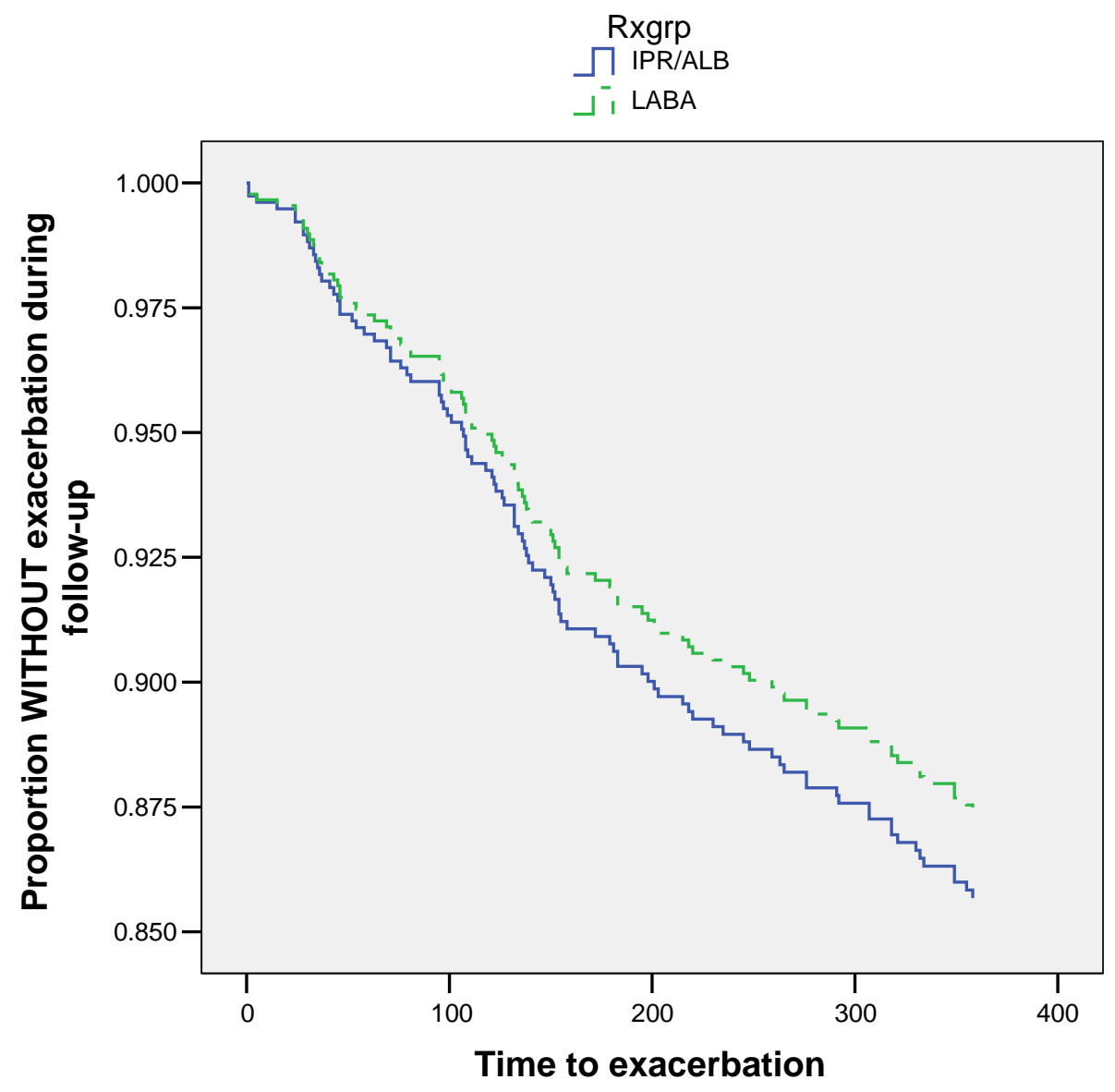


TABLE 28: Logistic regression of the impact of drug therapy (IPR/ALB or LABA) on probability of exacerbation

\begin{tabular}{|c|c|c|c|}
\hline \multirow[b]{2}{*}{ Variable } & \multicolumn{3}{|c|}{ DV: Probability of exacerbation } \\
\hline & Odds ratio $(S E)$ & $95 \% C I$ & $\begin{array}{c}p \\
\text { value }\end{array}$ \\
\hline \multicolumn{4}{|l|}{ Drug therapy (Ref: IPR/ALB) } \\
\hline LABA & $0.844(0.232)$ & $0.493-1.446$ & 0.538 \\
\hline \multicolumn{4}{|l|}{ Demographic } \\
\hline Age (years) & $1.017(0.016)$ & $0.986-1.049$ & 0.299 \\
\hline Gender (Ref: Male) & & & \\
\hline Female & $1.920(0.478)$ & $1.179-3.127$ & $0.009^{*}$ \\
\hline Race (Ref: non-White) & & & \\
\hline White & $1.697(1.385)$ & $0.343-8.401$ & 0.517 \\
\hline State (Ref: WV ) & & & \\
\hline KY & $1.125(0.331)$ & $0.632-2.002$ & 0.689 \\
\hline County_Smokerate (Ref: $<31 \%)$ & & & \\
\hline$>=31 \%$ & $1.381(0.413)$ & $0.768-2.482$ & 0.281 \\
\hline \multicolumn{4}{|l|}{ Overall comorbidity } \\
\hline D'Hoore-CCI & $0.926(0.074)$ & $0.792-1.083$ & 0.334 \\
\hline Number of other chronic conditions & $0.762(0.077)$ & $0.625-0.930$ & $0.007 *$ \\
\hline \multicolumn{4}{|l|}{ COPD severity } \\
\hline \# of inhaled SABA canisters & $1.025(0.022)$ & $0.983-1.069$ & 0.252 \\
\hline$\#$ of other SABD prescriptions & $0.994(0.036)$ & $0.926-1.068$ & 0.878 \\
\hline \# of OCS prescriptions & $1.082(0.062)$ & $0.967-1.210$ & 0.168 \\
\hline Use of nebulized SABD & $1.194(0.404)$ & $0.615-2.318$ & 0.601 \\
\hline $\begin{array}{r}\text { \# of Hosp/ER visits for COPD-related } \\
\text { comorbid disorders }\end{array}$ & $1.500(0.144)$ & $1.242-1.812$ & $0.000^{*}$ \\
\hline $\begin{array}{r}\text { \# of Physician visits for COPD-related } \\
\text { comorbid disorders }\end{array}$ & $1.021(0.041)$ & $0.944-1.105$ & 0.599 \\
\hline Presence of asthma & $1.167(0.443)$ & $0.555-2.455$ & 0.684 \\
\hline
\end{tabular}

$* \mathrm{p}<0.05$

Log likelihood $=-250.56 ; \chi^{2}=49.86 ; \mathrm{p}=0.000 *$

Pseudo R ${ }^{2}$ : 9.1\% 
TABLE 29: Zero-inflated Poisson regression model of the impact of drug therapy (IPR/ALB or LABA) on the frequency of exacerbations

\begin{tabular}{|c|c|c|c|}
\hline \multirow[b]{2}{*}{ Variable } & \multicolumn{3}{|c|}{ DV: Number of exacerbations } \\
\hline & Coeff (SE) & $95 \% C I$ & $\begin{array}{c}p \\
\text { value }\end{array}$ \\
\hline \multicolumn{4}{|l|}{ Drug therapy (Ref: IPR/ALB) } \\
\hline LABA & $0.209(0.284)$ & $-0.347-0.766$ & 0.461 \\
\hline \multicolumn{4}{|l|}{ Demographic } \\
\hline Age (years) & $0.049(0.017)$ & $0.016-0.081$ & $0.003 *$ \\
\hline Gender (Ref: Male) & & & \\
\hline Female & $0.254(0.321)$ & $-0.375-0.884$ & 0.428 \\
\hline Race (Ref: non-White) & & & \\
\hline White & $0.818(0.482)$ & $-0.126-1.762$ & 0.090 \\
\hline State (Ref: WV ) & & & \\
\hline $\mathrm{KY}$ & $0.446(0.269)$ & $-0.082-0.973$ & 0.098 \\
\hline County_Smokerate (Ref: $<31 \%$ ) & & & \\
\hline$>=31 \%$ & $-0.774(0.275)$ & $-1.313--0.235$ & $0.005 *$ \\
\hline \multicolumn{4}{|l|}{ Overall comorbidity } \\
\hline D’Hoore-CCI & $0.020(0.088)$ & $-0.152-0.192$ & 0.817 \\
\hline Number of other chronic conditions & $-0.251(0.091)$ & $-0.429--0.073$ & $0.006^{*}$ \\
\hline \multicolumn{4}{|l|}{ COPD severity } \\
\hline \# of inhaled SABA canisters & $0.034(0.016)$ & $0.004-0.065$ & $0.029 *$ \\
\hline \# of other SABD prescriptions & $-0.010(0.031)$ & $-0.072-0.051$ & 0.445 \\
\hline \# of OCS prescriptions & $-0.091(0.047)$ & $-0.183-0.001$ & 0.748 \\
\hline Use of nebulized SABD & $-0.211(0.277)$ & $-0.754-0.331$ & 0.054 \\
\hline $\begin{array}{r}\text { \# of Hosp/ER visits for COPD-related } \\
\text { comorbid disorders }\end{array}$ & $0.262(0.072)$ & $0.120-0.403$ & $0.000 *$ \\
\hline $\begin{array}{r}\text { \# of Physician visits for COPD-related } \\
\text { comorbid disorders }\end{array}$ & $0.056(0.040)$ & $0.162--0.022$ & 0.162 \\
\hline Presence of asthma & $0.207(0.349)$ & $-0.477-0.892$ & 0.552 \\
\hline
\end{tabular}


TABLE 30: Two part semi-logarithmic regression of the impact of drug therapy (IPR/ALB or LABA) on total COPD-related costs

\begin{tabular}{|c|c|c|}
\hline \multirow[b]{2}{*}{ Variable } & \multicolumn{2}{|c|}{ DV: Total COPD-related costs } \\
\hline & $\begin{array}{l}\beta \text { coefficient } \\
(S E)\end{array}$ & $\begin{array}{c}p \\
\text { value }\end{array}$ \\
\hline \multicolumn{3}{|l|}{ Drug therapy (Ref: IPR/ALB) } \\
\hline LABA & $0.124(0.098)$ & 0.206 \\
\hline \multicolumn{3}{|l|}{ Demographic } \\
\hline Age (years) & $0.018(0.006)$ & $0.002 *$ \\
\hline Gender (Ref: Male) & & \\
\hline Female & $0.105(0.088)$ & 0.230 \\
\hline Race (Ref: non-White) & & \\
\hline White & $0.058(0.244)$ & 0.811 \\
\hline State (Ref: WV ) & & \\
\hline $\mathrm{KY}$ & $0.101(0.107)$ & 0.344 \\
\hline County_Smokerate (Ref: <31\%) & & \\
\hline$>=31 \%$ & $0.057(0.107)$ & 0.592 \\
\hline \multicolumn{3}{|l|}{ Overall comorbidity } \\
\hline D’Hoore-CCI & $0.030(0.028)$ & 0.286 \\
\hline Number of other chronic conditions & $-0.034(0.036)$ & 0.314 \\
\hline \multicolumn{3}{|l|}{ COPD severity } \\
\hline \# of inhaled SABA canisters & $0.025(0.008)$ & $0.003 *$ \\
\hline \# of other SABD prescriptions & $0.032(0.013)$ & $0.016^{*}$ \\
\hline \# of OCS prescriptions & $-0.002(0.026)$ & 0.952 \\
\hline Use of nebulized SABD & $0.392(0.133)$ & $0.003 *$ \\
\hline $\begin{array}{r}\text { \# of Hosp/ER visits for COPD-related comorbid } \\
\text { disorders }\end{array}$ & $0.130(0.040)$ & $0.001 *$ \\
\hline $\begin{array}{r}\text { \# of Physician visits for COPD-related } \\
\text { comorbid disorders }\end{array}$ & $0.041(0.016)$ & $0.010^{*}$ \\
\hline Presence of asthma & $0.077(0.155)$ & 0.617 \\
\hline
\end{tabular}


TABLE 31: Two part semi-logarithmic regression of the impact of drug therapy (IPR/ALB or LABA) on COPD-related prescription drug costs

\begin{tabular}{|c|c|c|}
\hline \multirow[b]{2}{*}{ Variable } & \multicolumn{2}{|c|}{$\begin{array}{l}\text { DV: COPD-related } \\
\text { prescription drug costs }\end{array}$} \\
\hline & $\begin{array}{l}\beta \text { coefficient } \\
(S E)\end{array}$ & $\begin{array}{c}p \\
\text { value }\end{array}$ \\
\hline \multicolumn{3}{|l|}{ Drug therapy (Ref: IPR/ALB) } \\
\hline LABA & $0.216(0.062)$ & $0.001 *$ \\
\hline \multicolumn{3}{|l|}{ Demographic } \\
\hline Age (years) & $0.005(0.004)$ & 0.183 \\
\hline Gender (Ref: Male) & & \\
\hline Female & $0.045(0.055)$ & 0.411 \\
\hline Race (Ref: non-White) & & \\
\hline White & $0.145(0.154)$ & 0.346 \\
\hline State (Ref: WV ) & & \\
\hline $\mathrm{KY}$ & $0.196(0.067)$ & $0.004 *$ \\
\hline County_Smokerate (Ref: <31\%) & & \\
\hline$>=31 \%$ & $0.058(0.067)$ & 0.389 \\
\hline \multicolumn{3}{|l|}{ Overall comorbidity } \\
\hline D’Hoore-CCI & $0.017(0.017)$ & 0.335 \\
\hline Number of other chronic conditions & $-0.009(0.022)$ & 0.695 \\
\hline \multicolumn{3}{|l|}{ COPD severity } \\
\hline \# of inhaled SABA canisters & $0.036(0.005)$ & $0.000^{*}$ \\
\hline \# of other SABD prescriptions & $0.034(0.008)$ & $0.000^{*}$ \\
\hline \# of OCS prescriptions & $0.015(0.016)$ & 0.374 \\
\hline Use of nebulized SABD & $0.272(0.084)$ & $0.001 *$ \\
\hline $\begin{array}{r}\# \text { of Hosp/ER visits for COPD-related comorbid } \\
\text { disorders }\end{array}$ & $-0.008(0.025)$ & 0.742 \\
\hline $\begin{array}{r}\text { \# of Physician visits for COPD-related } \\
\text { comorbid disorders }\end{array}$ & $0.023(0.010)$ & $0.019^{*}$ \\
\hline Presence of asthma & $0.122(0.098)$ & 0.214 \\
\hline
\end{tabular}




\section{Discussion for Research Objectives 3 and 4}

The 2004 update to the GOLD guidelines suggested that long-acting beta-agonists were more effective and convenient for maintenance therapy than the short-acting anti-cholinergic, ipratropium (GOLD, 2004). However, a review of the literature showed the evidence for long-acting beta-agonists relative to ipratropium to be inconsistent. Therefore, Part 1 of Phase II compared initiation of monotherapy with long-acting beta-agonist relative to therapy with a combination of ipratropium/albuterol. The combination therapy with ipratropium/albuterol has been demonstrated to be comparable to using ipratropium alone for maintenance therapy in terms of both costs and effects. The former was chosen as the comparator in this study because of the difficulty in designating use of ipratropium alone as maintenance therapy from claims data.

The results of objective 3 showed that ipratropium was as effective as LABA in the proportions of recipients experiencing an exacerbation. These results are similar to results from Rennard et al. and Dahl et al. in which there were no differences in the proportion of patients with exacerbations requiring oral corticosteroids, but contrast those from a trial by Mahler et al. which showed a significantly longer time to an exacerbation relative to ipratropium (Rennard et al., 2001; Dahl et al., 2001; Mahler et al., 1999). In two studies using observational data such as the present study, a trend towards a lower hazard for a severe exacerbation (hospitalization) was seen for patients initiating therapy on salmeterol compared to those on ipratropium (Burney et al., 2003; Rascati et al., 2005). In both studies, the hazard ratio ranged from 0.76 to 0.82 . In our study, the hazard ratio was 0.87 . However, these results are not comparable as our study population was a relatively less severe population, while those in the other observational studies were at different severity levels. Additionally, our results assessed the impact not only on severe exacerbations but also on moderate exacerbations. There are no observational studies that have seen the effect of a long-acting beta-agonist relative to ipratropium in a moderately severe COPD population. In contrast to the above studies and our study, another study using the Pharmetrics database found ipratropium to have a significantly higher risk for a COPD-related $E R(O R=1.9)$ or hospitalization $(\mathrm{OR}=1.5)$ visit compared to salmeterol (Howard KB et al., 2001). However, the authors do mention the possibility that the ipratropium group was probably sicker. Neither the study design nor the analyses attempted to control the selection bias. 
The data source for the current study precluded relevant clinical measures of symptom scores, dyspnea scores, quality of life, and lung function. However, the data do provide information on COPD-related costs. Research has shown that those with poorer quality of life, and low lung function incur higher costs (Fan et al., 2002; Hilleman et al., 2000), and it is plausible that patients who incur higher costs have higher symptom and dyspnea scores. Hence, COPD-related costs, which can be considered as a summative measure representing these outcomes, were compared between the drug therapy groups. Results of objective 4 showed that both therapy groups incurred similar total COPD-related costs in the follow-up period. Although total COPD-related costs were not statistically different, recipients initiated on long-acting beta-agonists were found to incur 24\% higher COPD-related prescription drug costs.

The literature cites three pharmacoeconomic studies comparing COPD-related costs between patients using salmeterol versus ipratropium. One of the studies by Howard et al. mentioned above, which found ipratropium to have a higher risk of a COPD-related hospitalization or ER visit, found no difference in total COPD-related costs between the groups (Howard KB et al., 2001). Friedman et al. conducted a hypothetical analysis based on data from two different trials of ipratropium and salmeterol (Friedman et al., 2001). The trials found similar reductions in exacerbations for salmeterol and ipratropium (37\% vs. 33\%, respectively). The authors did not empirically assess, but estimated that salmeterol would have higher annual costs $(\$ 1,059)$ compared to ipratropium $(\$ 788)$. In our study, recipients initiated on long-acting beta-agonists had an annual cost of $\$ 2,291$ compared to $\$ 2,023$ for those on ipratropium. None of the aforementioned two studies individually examined cost components. A study by Hogan et al. used information from a clinical trial comparing ipratropium to formoterol on measures of $\mathrm{FEV}_{1}$ and quality of life to conduct a pharmacoeconomic analysis (Hogan et al., 2003). The major limitation of this analysis was that no a priori economic data were collected during the trial. Instead, only the cost of the study drugs and rescue medications were considered. The results showed those on formoterol to have incremental costs of $\$ 1,611$ per additional change in $\mathrm{FEV}_{1}$ and $\$ 25$ per additional change in units of the quality of life instrument. The results imply that patients on formoterol incurred higher COPD-related prescription drug costs compared to those on 
ipratropium. However, our study included other COPD-related drug classes when computing the cost for COPD-related prescription drugs.

As was mentioned earlier, COPD-related costs can be considered a summative measure representing outcomes of lung function, exercise capacity, dyspnea, symptom scores, and health-related quality of life. The result of no difference in total COPD-related costs can be explained by the equal efficacy of long-acting beta-agonists to ipratropium found on these measures in four clinical trials. Of the four trials, only one trial found significant differences on lung function. Salmeterol was shown to be statistically different from ipratropium at 4 and 6 hours after bronchodilator administration at the end of 12 weeks in change in $\mathrm{FEV}_{1} \mathrm{AUC}$ (area under curve) (Mahler et al., 1999). Another trial found a statistical but not a clinically relevant difference in normalized $\mathrm{FEV}_{1} \mathrm{AUC}$ (area under the curve) at 12 weeks between ipratropium and formoterol (Dahl et al., 2001).

A timed walking test is a measure of functional exercise capacity that indicates the level of $\underline{d y s p n e a}$. Both salmeterol trials have found no differences between salmeterol and ipratropium in either the distance walked or the post-walk dyspnea scores using the 6 minute walking test (Mahler et al., 1999; Rennard et al., 2001). Formoterol also showed similar results on exercise capacity as measured using the shuttle walking test, with 41 percent in the formoterol group and 38 percent of patients in the ipratropium group reaching a clinically significant improvement of $\geq 30 \mathrm{~m}$ after 12 weeks (difference between groups not statistically significant) (Wadbo et al., 2002). Dyspnea scores using the Baseline Dyspnea Index (BDI) and the Transitional Dyspnea Index (TDI) did not differ significantly between salmeterol and ipratropium groups in both trials. Only one formoterol trial assessed daytime and nighttime dyspnea scores using a symptom scale, and also found insignificant differences compared with ipratropium (Wadbo et al., 2002).

In terms of HRQol, no differences were seen in the total Chronic Respiratory Questionnaire (CRQ) score between salmeterol and ipratropium (Mahler et al., 1999; Rennard et al., 2001). Formoterol again showed inconsistent results on the St. George's Respiratory Questionnaire (SGRQ). The trial conducted by Dahl et al. found that formoterol differed statistically from ipratropium by -3.79 units on total SGRQ score, less than the minimum clinically important difference of four units (Dahl et al., 2001). The other 
formoterol trial reported no statistically significant differences between groups (Wadbo et al., 2002).

Patients on ipratropium and long-acting beta-agonists reported similar symptom scores at the end of 12 weeks in 3 trials (Mahler et al., 1999; Rennard et al., 2001; Wadbo et al., 2002). In one trial, the daily symptom score was 0.3 units lower in the formoterol group (only for $12 \mu \mathrm{g}$ and not $24 \mu \mathrm{g}$ ) compared to the ipratropium group that was statistically significant (Dahl et al., 2001). Increase in symptoms necessitates increased use of rescue medications, measured as daily number of puffs or inhalations. Therefore, the trials that showed no differences in symptom scores also showed no differences in daily number of puffs or inhalations of rescue medication required (Mahler et al., 1999; Rennard et al., 2001; Wadbo et al., 2002), while the formoterol trial showed significant differences (Dahl et al., 2001). Dahl et al. showed the mean daily number of puffs of inhaled albuterol during the treatment period to be $1.2,1.7,2.5$, and 2.0 in the $12 \mu \mathrm{g}, 24 \mu \mathrm{g}$ formoterol, ipratropium and placebo group, respectively (Dahl et al., 2001). Both doses of formoterol produced a significant reduction in the need for rescue medication versus ipratropium $(\mathrm{p} \leq 0.014)$. 
Part 2

The second part of phase II compares outcomes of exacerbations and costs between

recipients initiating combination therapy with an inhaled corticosteroid and long-acting betaagonist (ICS/LABA), inhaled corticosteroid monotherapy (ICS alone), inhaled long-acting beta-agonist monotherapy (LABA alone), and those on short-acting bronchodilators (SABD). Table 32 outlines the extraction process from raw data to obtain the study sample.

TABLE 32: Sample extraction for Phase II - Part 2

\begin{tabular}{|c|c|c|}
\hline Step & Outcome WV & Outcome KY \\
\hline $\begin{array}{l}\text { 1. \# with a Rx claim for combination } \\
\text { product of ICS/LABA, ICS, LABA, or } \\
\text { SABD during: WV- Jul 1, } 1998 \text { to Jun 30, } \\
2002 \text { and KY-Jan } 1,1999 \text { to Dec } 31,2002\end{array}$ & $\begin{array}{l}\mathrm{A}=159,514 \\
-\mathrm{ICS} / \mathrm{LABA}=6,328 \\
-\mathrm{ICS} \text { alone }=26,620 \\
-\mathrm{LABA} \text { alone }=11,053 \\
-\mathrm{SABD}=115,513\end{array}$ & $\begin{array}{l}\mathrm{A}=323,567 \\
-\mathrm{ICS} / \mathrm{LABA}=16,938 \\
-\mathrm{ICS} \text { alone }=60,407 \\
-\mathrm{LABA} \text { alone }=21,991 \\
-\mathrm{SABD}=224,231\end{array}$ \\
\hline \multicolumn{3}{|c|}{$\begin{array}{l}\text { 2. The date of first chronologically occurring prescription claim was defined as an index date for } \\
\text { each person. The drug therapy groups represent numbers with respective prescription claim at } \\
\text { index date. }\end{array}$} \\
\hline $\begin{array}{l}\text { 3. \# of A between } 35-64 \text { years, continuously } \\
\text { eligible for Medicaid and not enrolled in } \\
\text { managed care at anytime during study } \\
\text { period }\end{array}$ & $\begin{array}{l}\mathrm{B}=29,281 \\
-\mathrm{ICS} / \mathrm{LABA}=2,307 \\
-\mathrm{ICS} \text { alone }=6,703 \\
-\mathrm{LABA} \text { alone }=3,634 \\
-\mathrm{SABD}=16,637\end{array}$ & $\begin{array}{l}\mathrm{B}=56,128 \\
-\mathrm{ICS} / \mathrm{LABA}=5,500 \\
-\mathrm{ICS} \text { alone }=12,460 \\
-\mathrm{LABA} \text { alone }=6,806 \\
-\mathrm{SABD}=31,362\end{array}$ \\
\hline $\begin{array}{l}\text { 4. \# of B with prescription drug patterns } \\
\text { specified in study criteria. }\end{array}$ & $\begin{array}{l}\mathrm{C}=14,477 \\
-\mathrm{ICS} / \mathrm{LABA}=962 \\
-\mathrm{ICS} \text { alone }=3,298 \\
-\mathrm{LABA} \text { alone }=853 \\
-\mathrm{SABD}=9,364\end{array}$ & $\begin{array}{l}\mathrm{C}=27,450 \\
-\mathrm{ICS} / \mathrm{LABA}=2,439 \\
-\mathrm{ICS} \text { alone }=5,771 \\
-\mathrm{LABA} \text { alone }=1,436 \\
-\mathrm{SABD}=17,804\end{array}$ \\
\hline $\begin{array}{l}\text { 5. \# of } \mathrm{C} \text { without diagnosis of cystic fibrosis, } \\
\text { fibrosis due to tuberculosis, respiratory } \\
\text { cancer, and bronchiectasis during study } \\
\text { period }\end{array}$ & $\begin{array}{l}\mathrm{D}=14,153 \\
-\mathrm{ICS} / \mathrm{LABA}=922 \\
-\mathrm{ICS} \text { alone }=3,210 \\
-\mathrm{LABA} \text { alone }=824 \\
-\mathrm{SABD}=9,197\end{array}$ & $\begin{array}{l}\mathrm{D}=26,961 \\
-\mathrm{ICS} / \mathrm{LABA}=2,393 \\
-\mathrm{ICS} \text { alone }=5,645 \\
-\mathrm{LABA} \text { alone }=1,404 \\
-\mathrm{SABD}=17,519\end{array}$ \\
\hline $\begin{array}{l}\text { 6. \# of D with at least one hospitalization or } \\
\text { ER visit or at least two outpatient } \\
\text { encounters on different dates with a } \\
\text { primary or secondary diagnosis of COPD } \\
\text { in pre-index period }\end{array}$ & $\begin{array}{l}\mathrm{E}=2,869 \\
-\mathrm{ICS} / \mathrm{LABA}=331 \\
-\mathrm{ICS} \text { alone }=1,060 \\
-\mathrm{LABA} \text { alone }=248 \\
-\mathrm{SABD}=1,230\end{array}$ & $\begin{array}{l}\mathrm{E}=5,246 \\
-\mathrm{ICS} / \mathrm{LABA}=654 \\
-\mathrm{ICS} \text { alone }=1,709 \\
-\mathrm{LABA} \text { alone }=394 \\
-\mathrm{SABD}=2,489\end{array}$ \\
\hline
\end{tabular}


7. \# of E who had at least one additional prescription claim of index drug therapy in follow-up period.

8. Final study sample size

$\begin{array}{ll}\mathrm{F}=1,184 & \mathrm{~F}=2,274 \\ -\mathrm{ICS} / \mathrm{LABA}=236 & -\mathrm{ICS} / \mathrm{LABA}=494 \\ -\mathrm{ICS} \text { alone }=645 & -\mathrm{ICS} \text { alone }=1,174 \\ -\mathrm{LABA} \text { alone }=150 & \text {-LABA alone = 264 } \\ -\mathrm{SABD}=153 & -\mathrm{SABD}=342\end{array}$

$\mathrm{I}=3,458$

$-\mathrm{ICS} / \mathrm{LABA}=730$

-ICS alone $=1,819$

$-\mathrm{LABA}$ alone $=414$

$-\mathrm{SABD}=495$

Four drug therapy cohorts were formed for Part 2 of Phase II. Three primary comparisons were of interest:

1. ICS/LABA vs. SABD

2. ICS monotherapy vs. SABD

3. ICS/LABA vs. LABA

Two combination ICS/LABA products are now available: combination fluticasone/salmeterol commonly called Advair ${ }^{\circledR}$, and combination budesonide/formoterol commonly called Symbicort $\AA$. In the present study, only Advair $\AA$ was available during the the study time period, and consequently ICS/LABA refers to the combination of fluticasone/salmeterol.

Since the study is observational in nature, there is an inherent selection bias in the creation of these drug therapy cohorts. Hence, propensity scores were used to account for selection bias. The logistic regression model predicting the probability of being in the drug cohort with an ICS is presented in table B1, B2, and B3 in appendix B for comparison 1, 2, and 3 respectively. The results for objective 5, 6, and 7 are presented for each of the three above comparisons.

ICS/LABA vs. SABD: Table 33 outlines the comparison of characteristics of the study sample before and after matching for the analyses comparing ICS/LABA vs. SABD. A total of 173 matched pairs were obtained. Before matching, the groups differed significantly on demographic and comorbid characteristics. Recipients who were prescribed ICS/LABA were more likely to be younger females, and reside in counties with a lower prevalence of 
smoking. Although the D'Hoore Charlson comorbidity index showed no differences, recipients in the ICS/LABA group had a higher mean number of other chronic conditions (1.9 vs. 1.6). Recipients with ICS/LABA were also more likely to have a hospitalization or ER visit for COPD or a COPD-related disorder in the pre-index period compared to the SABD group (52.9\% vs. $44.2 \%)$, and had a slightly higher but significant number of prescriptions of oral corticosteroids (1.2 vs. 1.1). As expected, recipients with concomitant asthma were also more likely to have been prescribed ICS/LABA (23.0\% vs. $14.6 \%)$. Recipients in the SABD group had a higher number of canisters of SABA and IPR, and a higher number of prescriptions of other dose forms of short-acting bronchodilators. However, this increased use may be a reflection of the study design, since those in the SABD group were required to have at least two SABD prescriptions in the pre-index period of which one was for IPR. This was done in order to avoid including a relatively mild COPD population who use SABD on an acute as-needed basis. The matching process was successful in balancing the covariates at baseline as can be seen from the significance tests after matching.

ICS alone vs. SABD: Table 34 outlines the comparison of characteristics of the study sample before and after matching for the analyses comparing ICS alone vs. SABD. A total of 335 matched pairs were obtained. Recipients using ICS alone were more likely to be younger females, and reside in counties with a lower prevalence of smoking. Recipients with ICS alone also had a significantly higher D'Hoore CCI as well as number of other chronic conditions. Also, a significantly higher proportion of those using ICS alone had a hospitalization or ER visit for COPD or COPD-related comorbid disorders $(53.6 \%$ vs. $44.2 \%)$, and were found to have asthma ( $21.8 \%$ vs. $14.6 \%)$. Similar to the comparison of ICS/LABA vs. SABD, recipients in the SABD group had a higher number of canisters of SABA and IPR, and a higher number of prescriptions of other dose forms of short-acting bronchodilators compared to those using ICS alone. Of note however, a significantly higher proportion of recipients in the SABD group used home oxygen therapy (18.4\% vs. $13.3 \%)$. The matching process was not completely successful in balancing the covariates at baseline as can be seen from the significance tests after matching. Specifically, recipients in the SABD group still had a significantly higher number of canisters of SABA (4.7 vs. 3.9) and 
IPR (4.2 vs. 3.5) after matching. However, the use of home oxygen therapy was balanced between the groups. Hence, the difference in the use of SABA and IPR canisters was considered an artifact of the study design rather than to any differences in severity between the groups. However, the researcher conducted a separate analysis on the full sample (1819+495) using the propensity score quintile, and number of canisters of SABA and IPR in the regression model (data not shown). Since the results were no different from that of the analysis of the matched sample, only the analysis of the matched sample is shown.

ICS/LABA vs. LABA alone: Table 35 outlines the comparison of characteristics of the study sample before and after matching for the analyses comparing ICS/LABA vs. LABA alone. A total of 240 matched pairs were obtained. Recipients prescribed combination ICS/LABA were more likely to be younger females, and have asthma. However, few differences in COPD severity measures were seen between therapy groups. Specifically, recipients being prescribed ICS/LABA had a slightly higher mean number of prescriptions for oral corticosteroids (1.2 vs. 1.1), and were more likely to have used nebulized SABD (29.3\% vs. $20.5 \%$ ) compared to those using LABA alone. No differences were seen in the use of hospitalization/ER visit for COPD or a COPD related comorbid disorder (52.9\% vs. $48.6 \%$ ). However, recipients using ICS/LABA had a significantly higher mean number of physician visits for COPD or COPD-related disorders (4.0 vs. 3.6). The matching process was successful in balancing the covariates at baseline as can be seen from the significance tests after matching. 
TABLE 33: Baseline characteristics of study sample by drug therapy status (ICS/LABA vs. SABD: Phase II-Part 2)

\begin{tabular}{|c|c|c|c|c|c|c|}
\hline \multirow[b]{2}{*}{ Characteristic } & \multicolumn{3}{|c|}{ Before Matching } & \multicolumn{3}{|c|}{ After Matching } \\
\hline & $\begin{array}{c}\text { ICS/LABA } \\
(\mathrm{N}=730)\end{array}$ & $\begin{array}{c}\text { SABD } \\
(\mathrm{N}=495)\end{array}$ & $\begin{array}{c}\text { Test } \\
\text { Statistic } \\
\text { (p-value) }\end{array}$ & $\begin{array}{c}\text { ICS/LABA } \\
(\mathrm{N}=173)\end{array}$ & $\begin{array}{c}\text { SABD } \\
(\mathrm{N}=173)\end{array}$ & $\begin{array}{c}\text { Test } \\
\text { Statistic } \\
\text { (p-value) }\end{array}$ \\
\hline \multicolumn{7}{|l|}{ Demographic } \\
\hline $\begin{array}{l}\text { Age, mean years } \\
\text { (SD) }\end{array}$ & $51(7.9)$ & $53(7.4)$ & $\begin{array}{c}5.449^{1} \\
(0.000)^{*}\end{array}$ & $52(7.2)$ & $52(8.1)$ & $\begin{array}{c}0.152^{4} \\
(0.879)\end{array}$ \\
\hline Female, \% (n) & $\begin{array}{l}66.9 \% \\
(486)\end{array}$ & $\begin{array}{l}58.0 \% \\
(286)\end{array}$ & $\begin{array}{c}9.875^{2} \\
(0.002)^{*}\end{array}$ & $\begin{array}{l}60.7 \% \\
(105)\end{array}$ & $\begin{array}{l}60.7 \% \\
(105)\end{array}$ & $\begin{array}{l}0.000^{5} \\
(1.000)\end{array}$ \\
\hline White, \% (n) & $\begin{array}{l}96.6 \% \\
(627)\end{array}$ & $\begin{array}{l}98.2 \% \\
(439)\end{array}$ & $\begin{array}{l}2.546^{2} \\
(0.111)\end{array}$ & $\begin{array}{l}96.5 \% \\
(167)\end{array}$ & $\begin{array}{c}96.5 \% \\
(167)\end{array}$ & $\begin{array}{c}0.000^{5} \\
(1.000)\end{array}$ \\
\hline State $(\mathrm{KY}), \%(\mathrm{n})$ & $\begin{array}{l}67.7 \% \\
(494)\end{array}$ & $\begin{array}{l}69.1 \% \\
(342)\end{array}$ & $\begin{array}{l}0.274^{2} \\
(0.600)\end{array}$ & $\begin{array}{l}62.4 \% \\
(108)\end{array}$ & $\begin{array}{l}65.9 \% \\
(114)\end{array}$ & $\begin{array}{c}0.430^{5} \\
(0.586)\end{array}$ \\
\hline $\begin{array}{l}\text { Countysmokerate } \\
>=31 \%, \% \text { (n) }\end{array}$ & $\begin{array}{l}60.6 \% \\
(442)\end{array}$ & $\begin{array}{l}70.9 \% \\
(351)\end{array}$ & $\begin{array}{c}13.872^{2} \\
(0.000)^{*}\end{array}$ & $\begin{array}{l}65.9 \% \\
(114)\end{array}$ & $\begin{array}{l}65.9 \% \\
(114)\end{array}$ & $\begin{array}{c}0.000^{5} \\
(1.000)\end{array}$ \\
\hline $\begin{array}{l}\text { Overall } \\
\text { Comorbidity }\end{array}$ & & & & & & \\
\hline $\begin{array}{l}\mathrm{D}^{\prime} \text { Hoore-CCI } \\
\text { mean }(\mathrm{SD})\end{array}$ & $1.5(1.8)$ & $1.3(1.8)$ & $\begin{array}{l}-1.315^{3} \\
(0.189)\end{array}$ & $1.2(1.8)$ & $1.4(1.9)$ & $\begin{array}{l}-1.053 \\
(0.293)\end{array}$ \\
\hline $\begin{array}{l}\text { Number of other } \\
\text { chronic } \\
\text { conditions }^{\mathrm{b}} \\
\text { mean (SD) }\end{array}$ & $1.9(1.3)$ & $1.6(1.3)$ & $\begin{array}{l}-7.029^{3} \\
(0.000)^{*}\end{array}$ & $1.8(1.3)$ & $1.9(1.3)$ & $\begin{array}{l}-0.352 \\
(0.725)\end{array}$ \\
\hline COPD severity & & & & & & \\
\hline $\begin{array}{l}\# \text { of inhaled } \\
\text { SABA }^{c} \text { canisters, } \\
\text { mean }(\mathrm{SD})\end{array}$ & $3.9(7.4)$ & $5.4(6.9)$ & $\begin{array}{c}6.250^{1} \\
(0.006)^{*}\end{array}$ & $3.6(6.8)$ & $3.8(5.0)$ & $\begin{array}{l}-1.932^{4} \\
(0.053)\end{array}$ \\
\hline $\begin{array}{l}\text { \# of inhaled IPR } \\
\text { canisters, } \\
\text { mean (SD) }\end{array}$ & $2.3(5.0)$ & $5.9(6.6)$ & $\begin{array}{c}16.121^{1} \\
(0.000)^{*}\end{array}$ & $2.9(5.3)$ & $3.2(3.6)$ & $\begin{array}{l}-2.404^{4} \\
(0.016)^{*}\end{array}$ \\
\hline $\begin{array}{l}\text { \# of Rx of other } \\
\text { doseform } \\
\mathrm{SABD}^{\mathrm{c}} \text {, }\end{array}$ & $2.0(4.2)$ & $3.8(5.1)$ & $\begin{array}{c}7.897 \\
(0.000)^{*}\end{array}$ & $2.3(4.3)$ & $2.5(4.4)$ & $\begin{array}{l}-0.919^{4} \\
(0.358)\end{array}$ \\
\hline
\end{tabular}




\begin{tabular}{|c|c|c|c|c|c|c|}
\hline \multicolumn{7}{|l|}{ mean (SD) } \\
\hline $\begin{array}{l}\# \text { of } \mathrm{Rx} \text { of } \mathrm{OCS}^{\mathrm{d}}, \\
\text { mean }(\mathrm{SD})\end{array}$ & $1.2(2.4)$ & $1.1(2.6)$ & $\begin{array}{l}-2.421^{1} \\
(0.016)^{*}\end{array}$ & $1.0(2.2)$ & $1.1(2.3)$ & $\begin{array}{r}0.095^{4} \\
(0.924)\end{array}$ \\
\hline $\begin{array}{l}\text { Use of nebulized } \\
\mathrm{SABD}^{\mathrm{c}}, \%(\mathrm{n})\end{array}$ & $\begin{array}{c}29.3 \% \\
(214)\end{array}$ & $\begin{array}{l}29.3 \% \\
(145)\end{array}$ & $\begin{array}{c}0.000^{2} \\
(0.993)\end{array}$ & $\begin{array}{l}23.7 \% \\
(41)\end{array}$ & $\begin{array}{l}31.2 \% \\
(54)\end{array}$ & $\begin{array}{l}2.770^{5} \\
(0.124)\end{array}$ \\
\hline $\begin{array}{l}\text { Use of Hosp/ER } \\
\text { for COPD or } \\
\text { COPD-related } \\
\text { comorbid } \\
\text { disorders }{ }^{e}, \%(n)\end{array}$ & $\begin{array}{l}52.9 \% \\
(386)\end{array}$ & $\begin{array}{l}44.2 \% \\
(219)\end{array}$ & $\begin{array}{c}8.780^{2} \\
(0.003)^{*}\end{array}$ & $\begin{array}{c}50.9 \% \\
(88)\end{array}$ & $\begin{array}{c}50.3 \% \\
(87)\end{array}$ & $\begin{array}{l}0.010^{5} \\
(1.000)\end{array}$ \\
\hline $\begin{array}{l}\text { \# of Physician } \\
\text { visits for COPD } \\
\text { or COPD-related } \\
\text { comorbid } \\
\text { disorders } \\
\text { mean }(\mathrm{SD})\end{array}$ & $4.0(3.9)$ & $3.8(3.5)$ & $\begin{array}{l}-1.016^{2} \\
(0.310)\end{array}$ & $3.8(3.9)$ & $3.8(3.7)$ & $\begin{array}{l}-0.052^{4} \\
(0.959)\end{array}$ \\
\hline $\begin{array}{l}\text { Presence of } \\
\text { asthma, \% (n) }\end{array}$ & $\begin{array}{c}23.0 \% \\
(168)\end{array}$ & $\begin{array}{c}14.6 \% \\
(72)\end{array}$ & $\begin{array}{l}13.428^{2} \\
(0.000)^{*}\end{array}$ & $\begin{array}{c}15.0 \% \\
(26)\end{array}$ & $\begin{array}{c}16.8 \% \\
(29)\end{array}$ & $\begin{array}{c}0.230^{5} \\
(0.749)\end{array}$ \\
\hline $\begin{array}{l}\text { Use of Home } \\
\text { oxygen therapy, } \\
\%(n)\end{array}$ & $\begin{array}{l}17.1 \% \\
(125)\end{array}$ & $\begin{array}{l}18.4 \% \\
(91)\end{array}$ & $\begin{array}{l}0.323^{2} \\
(0.570)\end{array}$ & $\begin{array}{c}16.8 \% \\
(29)\end{array}$ & $\begin{array}{c}15.6 \% \\
(27)\end{array}$ & $\begin{array}{c}0.010^{5} \\
(0.878)\end{array}$ \\
\hline \multicolumn{7}{|c|}{$\begin{array}{l}\text { *p }<0.05 \\
\text { a Excludes asthma } \\
\text { b Includes hypercholesterolemia, hypertension, other heart disease, AIDS, rheumatoid arthritis, osteoarthritis, } \\
\text { gall bladder disease, depression and anxiety, and schizophrenia. } \\
\text { c Short-acting beta-agonist: includes levalbuterol, albuterol, metaproterenol, bitolterol, terbutaline. } \\
{ }^{\mathrm{d}} \text { Short-acting bronchodilators: includes levalbuterol, albuterol, metaproterenol, bitolterol, terbutaline, } \\
\text { theophylline, and ipratropium. } \\
\text { d }{ }^{\mathrm{O}} \text { Oral corticosteroids: includes prednisone, prednisolone, methylprednisolone, cortisone, hydrocortisone, } \\
\text { dexamethasone, betamethasone, oral triamcinolone, oral budesonide. } \\
\mathrm{e}^{\mathrm{e}} \text { Includes COPD, asthma, upper respiratory tract infections, lower respiratory tract infections, septicemia, } \\
\text { allergic rhinitis, other diseases of the lung, congestive heart failure, and disorders of fluid, electrolyte, and acid- } \\
\text { base imbalance. } \\
{ }^{1} \text { t statistic; }{ }^{2} \text { Chi-square test statistic; }{ }^{3} \text { Mann-Whitney U-statistic; }{ }^{4} \text { Wilcoxon signed rank test statistic; }{ }^{5} \text { McNemar } \\
\text { test statistic. }\end{array}$} \\
\hline
\end{tabular}


TABLE 34: Baseline characteristics of study sample by drug therapy status (ICS vs. SABD: Phase II-Part 2)

\begin{tabular}{|c|c|c|c|c|c|c|}
\hline \multirow[b]{2}{*}{ Characteristic } & \multicolumn{3}{|c|}{ Before Matching } & \multicolumn{3}{|c|}{ After Matching } \\
\hline & $\begin{array}{c}\text { ICS } \\
(\mathrm{N}=\mathbf{1 , 8 1 9})\end{array}$ & $\begin{array}{c}\text { SABD } \\
(\mathrm{N}=495)\end{array}$ & $\begin{array}{c}\text { Test } \\
\text { Statistic } \\
\text { (p-value) }\end{array}$ & $\begin{array}{c}\text { ICS } \\
(\mathrm{N}=335)\end{array}$ & $\begin{array}{c}\text { SABD } \\
(\mathrm{N}=335)\end{array}$ & $\begin{array}{c}\text { Test } \\
\text { Statistic } \\
\text { (p-value) }\end{array}$ \\
\hline \multicolumn{7}{|l|}{ Demographic } \\
\hline $\begin{array}{l}\text { Age, mean years } \\
\text { (SD) }\end{array}$ & $52(7.5)$ & $53(7.4)$ & $\begin{array}{c}4.028^{1} \\
(0.000)^{*}\end{array}$ & $53(7.1)$ & $53(7.7)$ & $\begin{array}{r}0.589^{4} \\
(0.556)\end{array}$ \\
\hline Female, \% (n) & $\begin{array}{l}64.0 \% \\
(1,159)\end{array}$ & $\begin{array}{l}58.0 \% \\
(286)\end{array}$ & $\begin{array}{c}6.009^{2} \\
(0.014)^{*}\end{array}$ & $\begin{array}{l}59.7 \% \\
(200)\end{array}$ & $\begin{array}{l}58.5 \% \\
(196)\end{array}$ & $\begin{array}{c}0.010^{5} \\
(0.816)\end{array}$ \\
\hline White, \% (n) & $\begin{array}{l}96.4 \% \\
(1,585)\end{array}$ & $\begin{array}{l}98.2 \% \\
(439)\end{array}$ & $\begin{array}{l}3.668^{2} \\
(0.055)\end{array}$ & $\begin{array}{c}96.5 \% \\
(167)\end{array}$ & $\begin{array}{c}96.5 \% \\
(167)\end{array}$ & $\begin{array}{c}0.330^{5} \\
(0.774)\end{array}$ \\
\hline State $(\mathrm{KY}), \%(\mathrm{n})$ & $\begin{array}{r}64.5 \% \\
(1,174)\end{array}$ & $\begin{array}{l}69.1 \% \\
(342)\end{array}$ & $\begin{array}{c}0.274^{2} \\
(0.600)\end{array}$ & $\begin{array}{l}63.9 \% \\
(214)\end{array}$ & $\begin{array}{l}65.1 \% \\
(218)\end{array}$ & $\begin{array}{l}0.120^{5} \\
(0.796)\end{array}$ \\
\hline $\begin{array}{l}\text { Countysmokerate } \\
>=31 \%, \% \text { (n) }\end{array}$ & $\begin{array}{r}64.9 \% \\
(1,180)\end{array}$ & $\begin{array}{l}70.9 \% \\
(351)\end{array}$ & $\begin{array}{c}13.872^{2} \\
(0.000)^{*}\end{array}$ & $\begin{array}{l}66.5 \% \\
(223)\end{array}$ & $\begin{array}{l}69.3 \% \\
(232)\end{array}$ & $\begin{array}{c}0.570^{5} \\
(0.504)\end{array}$ \\
\hline Overall Comorbidity & & & & & & \\
\hline $\begin{array}{l}\mathrm{D}^{\prime} \text { Hoore-CCI } \\
\text { mean }(\mathrm{SD})\end{array}$ & $1.5(1.8)$ & $1.3(1.8)$ & $\begin{array}{l}-2.051^{3} \\
(0.040)^{*}\end{array}$ & $1.4(1.8)$ & $1.4(1.8)$ & $\begin{array}{l}-1.200 \\
(0.841)\end{array}$ \\
\hline $\begin{array}{l}\text { Number of other } \\
\text { chronic conditions } \\
\text { mean }(\mathrm{SD})\end{array}$ & $1.9(1.3)$ & $1.6(1.3)$ & $\begin{array}{l}-5.543^{3} \\
(0.000)^{*}\end{array}$ & $1.6(1.3)$ & $1.7(1.3)$ & $\begin{array}{c}-0.952 \\
(0.341)\end{array}$ \\
\hline COPD severity & & & & & & \\
\hline $\begin{array}{l}\# \text { of inhaled } \mathrm{SABA}^{\mathrm{c}} \\
\text { canisters, mean (SD) }\end{array}$ & $4.2(6.9)$ & $5.4(6.9)$ & $\begin{array}{c}5.107^{1} \\
(0.000)^{*}\end{array}$ & $3.9(6.0)$ & $4.7(5.7)$ & $\begin{array}{c}-2.490^{4} \\
(0.012)^{*}\end{array}$ \\
\hline $\begin{array}{l}\text { \# of inhaled IPR } \\
\text { canisters, mean (SD) }\end{array}$ & $2.7(5.7)$ & $5.9(6.6)$ & $\begin{array}{c}16.768^{1} \\
(0.000)^{*}\end{array}$ & $3.5(5.5)$ & $4.2(4.6)$ & $\begin{array}{l}-3.904^{4} \\
(0.001)^{*}\end{array}$ \\
\hline $\begin{array}{l}\text { \# of Rx of other } \\
\text { doseform } \mathrm{SABD}^{\mathrm{c}} \text {, } \\
\text { mean (SD) }\end{array}$ & $2.3(4.4)$ & $3.8(5.1)$ & $\begin{array}{c}7.286 \\
(0.000)^{*}\end{array}$ & $3.1(5.0)$ & $3.1(4.7)$ & $\begin{array}{l}0.220^{4} \\
(0.826)\end{array}$ \\
\hline $\begin{array}{l}\# \text { of } \mathrm{Rx} \text { of } \mathrm{OCS}^{\mathrm{d}} \text {, } \\
\text { mean }(\mathrm{SD})\end{array}$ & $1.0(2.1)$ & $1.1(2.6)$ & $\begin{array}{l}-1.901^{1} \\
(0.057)\end{array}$ & $1.2(2.6)$ & $1.1(2.6)$ & $\begin{array}{l}2.028^{4} \\
(0.053)\end{array}$ \\
\hline
\end{tabular}




\begin{tabular}{|c|c|c|c|c|c|c|}
\hline $\begin{array}{l}\text { Use of nebulized } \\
\text { SABD }^{\mathrm{c}}, \%(\mathrm{n})\end{array}$ & $\begin{array}{l}26.5 \% \\
(214)\end{array}$ & $\begin{array}{l}29.3 \% \\
(145)\end{array}$ & $\begin{array}{l}1.539^{2} \\
(0.215)\end{array}$ & $\begin{array}{l}28.7 \% \\
(96)\end{array}$ & $\begin{array}{l}26.9 \% \\
(90)\end{array}$ & $\begin{array}{l}0.270^{5} \\
(0.664)\end{array}$ \\
\hline $\begin{array}{l}\text { Use of Hosp/ER for } \\
\text { COPD or COPD- } \\
\text { related comorbid } \\
\text { disorders }, \%(n)\end{array}$ & $\begin{array}{l}53.6 \% \\
(974)\end{array}$ & $\begin{array}{l}44.2 \% \\
(219)\end{array}$ & $\begin{array}{c}13.484^{2} \\
(0.000)^{*}\end{array}$ & $\begin{array}{c}47.2 \% \\
(158)\end{array}$ & $\begin{array}{c}46.6 \% \\
(156)\end{array}$ & $\begin{array}{l}0.030^{5} \\
(0.930)\end{array}$ \\
\hline $\begin{array}{l}\text { \# of Physician visits } \\
\text { for COPD or COPD- } \\
\text { related comorbid } \\
\text { disorders }{ }^{\mathrm{e}} \text {, mean (SD) }\end{array}$ & $3.8(3.6)$ & $3.8(3.5)$ & $\begin{array}{c}0.148^{2} \\
(0.883)\end{array}$ & $3.8(3.3)$ & $3.7(3.4)$ & $\begin{array}{l}0.559^{4} \\
(0.576)\end{array}$ \\
\hline $\begin{array}{l}\text { Presence of asthma, } \\
\% \text { (n) }\end{array}$ & $\begin{array}{l}21.8 \% \\
(396)\end{array}$ & $\begin{array}{c}14.6 \% \\
(72)\end{array}$ & $\begin{array}{c}12.588^{2} \\
(0.000)^{*}\end{array}$ & $\begin{array}{c}14.6 \% \\
(49)\end{array}$ & $\begin{array}{l}17.3 \% \\
(58)\end{array}$ & $\begin{array}{l}0.890^{5} \\
(0.402)\end{array}$ \\
\hline $\begin{array}{l}\text { Use of Home oxygen } \\
\text { therapy, \%(n) }\end{array}$ & $\begin{array}{l}13.3 \% \\
(242)\end{array}$ & $\begin{array}{c}18.4 \% \\
(91)\end{array}$ & $\begin{array}{c}8.150^{2} \\
(0.004)^{*}\end{array}$ & $\begin{array}{l}17.6 \% \\
(59)\end{array}$ & $\begin{array}{c}15.5 \% \\
(52)\end{array}$ & $\begin{array}{l}0.520^{5} \\
(0.538)\end{array}$ \\
\hline
\end{tabular}

$* \mathrm{p}<0.05$

${ }^{\mathrm{a}}$ Excludes asthma

${ }^{\mathrm{b}}$ Includes hypercholesterolemia, hypertension, other heart disease, AIDS, rheumatoid arthritis, osteoarthritis, gall bladder disease, depression and anxiety, and schizophrenia.

${ }^{\mathrm{c}}$ Short-acting beta-agonist: includes levalbuterol, albuterol, metaproterenol, bitolterol, terbutaline.

${ }^{\mathrm{d}}$ Short-acting bronchodilators: includes levalbuterol, albuterol, metaproterenol, bitolterol, terbutaline, theophylline, and ipratropium.

${ }^{\mathrm{d}}$ Oral corticosteroids: includes prednisone, prednisolone, methylprednisolone, cortisone, hydrocortisone, dexamethasone, betamethasone, oral triamcinolone, oral budesonide.

${ }^{\mathrm{e}}$ Includes COPD, asthma, upper respiratory tract infections, lower respiratory tract infections, septicemia, allergic rhinitis, other diseases of the lung, congestive heart failure, and disorders of fluid, electrolyte, and acidbase imbalance.

${ }^{1}$ t statistic; ${ }^{2}$ Chi-square test statistic; ${ }^{3}$ Mann-Whitney U-statistic; ${ }^{4}$ Wilcoxon signed rank test statistic; ${ }^{5} \mathrm{McNemar}$ test statistic. 
TABLE 35: Baseline characteristics of study sample by drug therapy status (ICS/LABA vs. LABA: Phase II-Part 2)

\begin{tabular}{|c|c|c|c|c|c|c|}
\hline \multirow[b]{2}{*}{ Characteristic } & \multicolumn{3}{|c|}{ Before Matching } & \multicolumn{3}{|c|}{ After Matching } \\
\hline & $\begin{array}{c}\text { ICS/LABA } \\
(\mathrm{N}=730)\end{array}$ & $\begin{array}{c}\text { LABA } \\
(\mathrm{N}=414)\end{array}$ & $\begin{array}{c}\text { Test } \\
\text { Statistic } \\
\text { (p-value) }\end{array}$ & $\begin{array}{c}\text { ICS/LABA } \\
(N=240)\end{array}$ & $\begin{array}{c}\text { LABA } \\
(\mathrm{N}=\mathbf{2 4 0})\end{array}$ & $\begin{array}{c}\text { Test } \\
\text { Statistic } \\
\text { (p-value) }\end{array}$ \\
\hline \multicolumn{7}{|l|}{ Demographic } \\
\hline $\begin{array}{l}\text { Age, mean years } \\
\text { (SD) }\end{array}$ & $51(7.9)$ & $52(7.5)$ & $\begin{array}{c}2.557^{1} \\
(0.011)^{*}\end{array}$ & $52(7.6)$ & $52(7.4)$ & $\begin{array}{l}-0.064^{4} \\
(0.949)\end{array}$ \\
\hline Female, \% (n) & $\begin{array}{l}66.9 \% \\
(486)\end{array}$ & $\begin{array}{l}59.8 \% \\
(247)\end{array}$ & $\begin{array}{c}5.693^{2} \\
(0.017)^{*}\end{array}$ & $\begin{array}{l}61.7 \% \\
(148)\end{array}$ & $\begin{array}{c}58.3 \% \\
(140)\end{array}$ & $\begin{array}{l}0.680^{5} \\
(0.471)\end{array}$ \\
\hline White, \% (n) & $\begin{array}{l}96.6 \% \\
(627)\end{array}$ & $\begin{array}{l}97.4 \% \\
(368)\end{array}$ & $\begin{array}{c}0.438^{2} \\
(0.508)\end{array}$ & $\begin{array}{l}97.9 \% \\
(235)\end{array}$ & $\begin{array}{l}98.3 \% \\
(236)\end{array}$ & $\begin{array}{l}0.110^{5} \\
(1.000)\end{array}$ \\
\hline State $(\mathrm{KY}), \%(\mathrm{n})$ & $\begin{array}{r}67.7 \% \\
(494)\end{array}$ & $\begin{array}{l}63.8 \% \\
(264)\end{array}$ & $\begin{array}{c}1.800^{2} \\
(0.180)\end{array}$ & $\begin{array}{l}60.0 \% \\
(144)\end{array}$ & $\begin{array}{l}63.8 \% \\
(153)\end{array}$ & $\begin{array}{c}0.770^{5} \\
(0.435)\end{array}$ \\
\hline $\begin{array}{l}\text { Countysmokerate } \\
>=31 \%, \% \text { (n) }\end{array}$ & $\begin{array}{l}60.6 \% \\
(442)\end{array}$ & $\begin{array}{l}64.3 \% \\
(266)\end{array}$ & $\begin{array}{l}1.536^{2} \\
(0.215)\end{array}$ & $\begin{array}{l}58.8 \% \\
(141)\end{array}$ & $\begin{array}{l}62.1 \% \\
(149)\end{array}$ & $\begin{array}{c}0.570^{5} \\
(0.509)\end{array}$ \\
\hline $\begin{array}{l}\text { Overall } \\
\text { Comorbidity }\end{array}$ & & & & & & \\
\hline $\begin{array}{l}\mathrm{D}^{\prime} \text { Hoore-CCI } \\
\text { mean }(\mathrm{SD})\end{array}$ & $1.5(1.8)$ & $1.5(1.8)$ & $\begin{array}{c}0.053^{3} \\
(0.958)\end{array}$ & $1.5(1.8)$ & $1.4(1.8)$ & $\begin{array}{c}0.490 \\
(0.624)\end{array}$ \\
\hline $\begin{array}{l}\text { Number of other } \\
\text { chronic } \\
\text { conditions }^{\text {b }} \\
\text { mean (SD) }\end{array}$ & $2.1(1.4)$ & $2.0(1.4)$ & $\begin{array}{l}-1.599^{3} \\
(0.110)\end{array}$ & $2.2(1.3)$ & $1.9(1.3)$ & $\begin{array}{c}1.147 \\
(0.252)\end{array}$ \\
\hline COPD severity & & & & & & \\
\hline $\begin{array}{l}\# \text { of inhaled } \\
\text { SABA }^{c} \text { canisters, } \\
\text { mean }(\mathrm{SD})\end{array}$ & $3.9(7.4)$ & $3.9(8.5)$ & $\begin{array}{l}-0.703^{3} \\
(0.482)\end{array}$ & $2.9(5.8)$ & $3.3(6.3)$ & $\begin{array}{l}-0.002^{4} \\
(0.999)\end{array}$ \\
\hline $\begin{array}{l}\text { \# of inhaled IPR } \\
\text { canisters, } \\
\text { mean (SD) }\end{array}$ & $2.3(5.0)$ & $2.6(6.1)$ & $\begin{array}{l}0.356^{3} \\
(0.722)\end{array}$ & $1.9(4.2)$ & $2.4(5.4)$ & $\begin{array}{l}-0.488^{4} \\
(0.625)\end{array}$ \\
\hline $\begin{array}{l}\text { \# of Rx of other } \\
\text { doseform } \\
\text { SABD }^{c}\end{array}$ & $2.0(4.3)$ & $2.0(4.2)$ & $\begin{array}{l}-0.225^{3} \\
(0.822)\end{array}$ & $1.8(4.1)$ & $2.3(4.5)$ & $\begin{array}{l}-1.690^{4} \\
(0.091)\end{array}$ \\
\hline
\end{tabular}




\begin{tabular}{|c|c|c|c|c|c|c|}
\hline mean (SD) & & & & & & \\
\hline $\begin{array}{l}\text { \# of Rx of OCS }{ }^{\mathrm{d}} \text {, } \\
\text { mean (SD) }\end{array}$ & $1.2(2.4)$ & $1.1(2.7)$ & $\begin{array}{c}-3.215^{3} \\
(0.001)^{*}\end{array}$ & $1.2(2.5)$ & $1.2(2.9)$ & $\begin{array}{l}1.715^{4} \\
(0.086)\end{array}$ \\
\hline $\begin{array}{l}\text { Use of nebulized } \\
\mathrm{SABD}^{\mathrm{c}}, \%(\mathrm{n})\end{array}$ & $\begin{array}{l}29.3 \% \\
(214)\end{array}$ & $\begin{array}{c}20.5 \% \\
(85)\end{array}$ & $\begin{array}{c}10.558^{2} \\
(0.001)^{*}\end{array}$ & $\begin{array}{c}17.1 \% \\
(41)\end{array}$ & $\begin{array}{c}20.8 \% \\
(50)\end{array}$ & $\begin{array}{l}1.530^{5} \\
(0.272)\end{array}$ \\
\hline $\begin{array}{l}\text { Use of Hosp/ER } \\
\text { for COPD or } \\
\text { COPD-related } \\
\text { comorbid } \\
\text { disorders }^{e}, \% \text { (n) }\end{array}$ & $\begin{array}{l}52.9 \% \\
(386)\end{array}$ & $\begin{array}{l}48.6 \% \\
(201)\end{array}$ & $\begin{array}{c}1.979^{2} \\
(0.160)\end{array}$ & $\begin{array}{l}51.7 \% \\
(124)\end{array}$ & $\begin{array}{l}47.9 \% \\
(115)\end{array}$ & $\begin{array}{l}0.740^{5} \\
(0.444)\end{array}$ \\
\hline $\begin{array}{l}\text { \# of Physician } \\
\text { visits for COPD } \\
\text { or COPD-related } \\
\text { comorbid } \\
\text { disorders } \\
\text { mean (SD) }\end{array}$ & $4.0(3.9)$ & $3.6(3.6)$ & $\begin{array}{c}-2.207^{3} \\
(0.027)^{*}\end{array}$ & $3.6(3.4)$ & $3.6(3.5)$ & $\begin{array}{l}-0.367^{4} \\
(0.713)\end{array}$ \\
\hline $\begin{array}{l}\text { Presence of } \\
\text { asthma, \% (n) }\end{array}$ & $\begin{array}{l}23.0 \% \\
(168)\end{array}$ & $\begin{array}{c}15.9 \% \\
(66)\end{array}$ & $\begin{array}{c}8.120^{1} \\
(0.004)^{*}\end{array}$ & $\begin{array}{c}18.3 \% \\
(44)\end{array}$ & $\begin{array}{c}16.3 \% \\
(39)\end{array}$ & $\begin{array}{l}0.410^{5} \\
(0.609)\end{array}$ \\
\hline $\begin{array}{l}\text { Use of Home } \\
\text { oxygen therapy, } \\
\%(n)\end{array}$ & $\begin{array}{l}17.1 \% \\
(125)\end{array}$ & $\begin{array}{c}15.2 \% \\
(63)\end{array}$ & $\begin{array}{c}0.699^{1} \\
(0.403)\end{array}$ & $\begin{array}{c}15.4 \% \\
(37)\end{array}$ & $\begin{array}{c}14.6 \% \\
(35)\end{array}$ & $\begin{array}{l}0.060^{5} \\
(0.902)\end{array}$ \\
\hline
\end{tabular}

$* \mathrm{p}<0.05$

a Excludes asthma

${ }^{\mathrm{b}}$ Includes hypercholesterolemia, hypertension, other heart disease, AIDS, rheumatoid arthritis, osteoarthritis, gall bladder disease, depression and anxiety, and schizophrenia.

${ }^{\mathrm{c}}$ Short-acting beta-agonist: includes levalbuterol, albuterol, metaproterenol, bitolterol, terbutaline.

${ }^{\mathrm{d}}$ Short-acting bronchodilators: includes levalbuterol, albuterol, metaproterenol, bitolterol, terbutaline, theophylline, and ipratropium.

${ }^{\mathrm{d}}$ Oral corticosteroids: includes prednisone, prednisolone, methylprednisolone, cortisone, hydrocortisone, dexamethasone, betamethasone, oral triamcinolone, oral budesonide.

${ }^{\mathrm{e}}$ Includes COPD, asthma, upper respiratory tract infections, lower respiratory tract infections, septicemia, allergic rhinitis, other diseases of the lung, congestive heart failure, and disorders of fluid, electrolyte, and acidbase imbalance.

${ }^{1}$ t statistic; ${ }^{2}$ Chi-square test statistic; ${ }^{3}$ Mann-Whitney U-statistic; ${ }^{4}$ Wilcoxon signed rank test statistic; ${ }^{5}$ McNemar test statistic. 
Research Objective 5: To compare the risk of a severe exacerbation among recipients with COPD who initiate therapy with a combination of an inhaled corticosteroid and a longacting beta-agonist, inhaled corticosteroid alone, inhaled long-acting beta-agonist alone, or only short-acting bronchodilators.

Table 36 shows the proportion experiencing an exacerbation between drug therapy cohorts for each of the three comparisons. Compared to those using SABD, inhaled corticosteroids alone or in combination with LABA did not appear to impact the risk of a severe exacerbation. In the comparison of ICS/LABA with SABD, the direction of the effect favored the combination product with an odds ratio of 0.75 (95\% CI: 0.384-1.441). However, in the comparison of ICS alone with SABD, the direction of the effect favored SABD with an odds ratio of 1.256 (95\% CI: 0.826-1.920). The addition of ICS to LABA monotherapy also did not have any effect on the risk of a severe exacerbation with an odds ratio of 1.261 (95\% CI 0.704-2.282).

Research Objective 6: To compare the frequency of moderate exacerbations among recipients with COPD who initiate therapy with a combination of an inhaled corticosteroid and a long-acting beta-agonist, inhaled corticosteroid alone, inhaled long-acting betaagonist alone, or only short-acting bronchodilators.

Table 37 compares the frequency of moderate exacerbations between the drug therapy cohorts for each of the three comparisons. There was no difference in the frequency of exacerbations between those using ICS/LABA vs. those using SABD or those using LABA monotherapy. However, in the comparison of ICS vs. SABD, a slightly higher but significant number of moderate exacerbations were found for those using ICS alone ( 0.28 vs. $0.18, \mathrm{p}=0.003)$.

Research Objective 7: To compare COPD-related costs among recipients with COPD who initiate therapy with a combination of an inhaled corticosteroid and a long-acting betaagonist, inhaled corticosteroid alone, inhaled long-acting beta-agonist alone, or only short-acting bronchodilators.

Table 38 compares COPD-related costs in the follow-up period between the drug therapy cohorts for each of the three comparisons. Inhaled corticosteroids either alone (ICS) or in 
combination (ICS/LABA) increased the total COPD-related costs relative to those using SABD by approximately $\$ 200$ to $\$ 300$. This increase was primarily driven by a difference in the prescription drug cost component. In the comparison of ICS/LABA vs. LABA alone, no differences were seen in overall COPD costs. However, COPD-related prescription drug costs were higher in the ICS/LABA group.

TABLE 36: Risk of a severe exacerbation in the follow-up period by drug therapy status (Phase II-Part 2)

\begin{tabular}{|c|c|c|c|}
\hline \multicolumn{3}{|l|}{ Outcome $^{\S}$} & \multirow{2}{*}{$\begin{array}{c}\text { Test Statistic } \\
\text { (p-value) } \\
*=\text { values }<0.05 \\
\end{array}$} \\
\hline \multirow[b]{2}{*}{ Severe exacerbations $(\%, n)$} & $\begin{array}{c}\text { ICS/LABA } \\
(\mathbf{N}=\mathbf{1 7 3})\end{array}$ & $\begin{array}{c}\text { SABD } \\
(\mathrm{N}=173)\end{array}$ & \\
\hline & $\begin{array}{c}12.1 \% \\
(21)\end{array}$ & $\begin{array}{c}15.6 \% \\
(27)\end{array}$ & \multirow[t]{2}{*}{$0.860^{1}(0.441)$} \\
\hline \multirow[t]{2}{*}{ Risk of a severe exacerbation } & \multicolumn{2}{|c|}{$\mathrm{OR}=0.750(0.384-1.441)$} & \\
\hline & $\begin{array}{c}\text { ICS } \\
(\mathrm{N}=335)\end{array}$ & $\begin{array}{c}\text { SABD } \\
(\mathrm{N}=335)\end{array}$ & \\
\hline Severe exacerbations $(\%, n)$ & $\begin{array}{c}19.1 \% \\
(53)\end{array}$ & $\begin{array}{c}15.8 \% \\
(64)\end{array}$ & $1.250^{1}(0.339)$ \\
\hline \multirow[t]{2}{*}{ Risk of a severe exacerbation } & \multicolumn{2}{|c|}{$\mathrm{OR}=1.256(0.826-1.920)$} & \\
\hline & $\begin{array}{c}\text { ICS/LABA } \\
(\mathrm{N}=\mathbf{2 4 0})\end{array}$ & $\begin{array}{c}\text { LABA } \\
(\mathrm{N}=\mathbf{2 4 0})\end{array}$ & \\
\hline Severe exacerbations $(\%, n)$ & $15.4 \%(37)$ & $12.9 \%(31)$ & $0.690^{1}(0.489)$ \\
\hline Risk of a severe exacerbation & \multicolumn{2}{|c|}{$\mathrm{OR}=1.261(0.704-2.282)$} & \\
\hline \multicolumn{4}{|c|}{$\begin{array}{l}\text { *p }<0.05 \\
\S \text { Analysis of difference in outcome controlled for variables included in the propensity score model: age, gender } \\
\text { race, countysmokerate, state, D'Hoore-CCI, number of other chronic conditions, number of inhaled SABA } \\
\text { canisters, number of inhaled IPR canisters, number of other doseforms of SABD, use of nebulized SABD, use } \\
\text { of Hosp/ER for COPD or COPD-related comorbid disorders, \# of physician visits for COPD or COPD-related } \\
\text { comorbid disorders, presence of asthma, and use of home oxygen therapy. } \\
{ }^{1} \text { McNemar test statistic }\end{array}$} \\
\hline
\end{tabular}


TABLE 37: Frequency of moderate exacerbations in follow-up period by drug therapy status (Phase II-Part 2)

\begin{tabular}{|c|c|c|c|}
\hline \multirow[t]{2}{*}{ Outcome $^{\S}$} & & & Test Statistic \\
\hline & $\begin{array}{c}\text { ICS/LABA } \\
(\mathrm{N}=173)\end{array}$ & $\begin{array}{c}\text { SABD } \\
(\mathrm{N}=173)\end{array}$ & \\
\hline \multirow[t]{2}{*}{ Moderate exacerbations, mean (SD) } & $0.31(0.9)$ & $0.18(0.6)$ & $1.692^{1}(0.091)$ \\
\hline & $\begin{array}{c}\text { ICS } \\
(\mathrm{N}=335)\end{array}$ & $\begin{array}{c}\text { SABD } \\
(\mathrm{N}=335)\end{array}$ & \\
\hline \multirow[t]{2}{*}{ Moderate exacerbations, mean (SD) } & $0.28(0.7)$ & $0.18(0.6)$ & $2.962^{1}(0.003)^{*}$ \\
\hline & $\begin{array}{c}\text { ICS/LABA } \\
(\mathrm{N}=240)\end{array}$ & $\begin{array}{c}\text { LABA } \\
(\mathrm{N}=\mathbf{2 4 0})\end{array}$ & \\
\hline Moderate exacerbations, mean (SD) & $0.2(0.5)$ & $0.3(0.7)$ & $-0.513^{1}(0.608)$ \\
\hline \multicolumn{4}{|c|}{$\begin{array}{l}{ }^{*} \mathrm{p}<0.05 \\
\S \text { Analysis of difference in outcome controlled for variables included in the propensity score model: age, gender } \\
\text { race, countysmokerate, state, D’Hoore-CCI, number of other chronic conditions, number of inhaled SABA } \\
\text { canisters, number of inhaled IPR canisters, number of other doseforms of SABD, use of nebulized SABD, use } \\
\text { of Hosp/ER for COPD or COPD-related comorbid disorders, \# of physician visits for COPD or COPD-related } \\
\text { comorbid disorders, presence of asthma, and use of home oxygen therapy. }\end{array}$} \\
\hline
\end{tabular}


TABLE 38: COPD-related costs in follow-up period by drug therapy status (Phase II-Part 2)

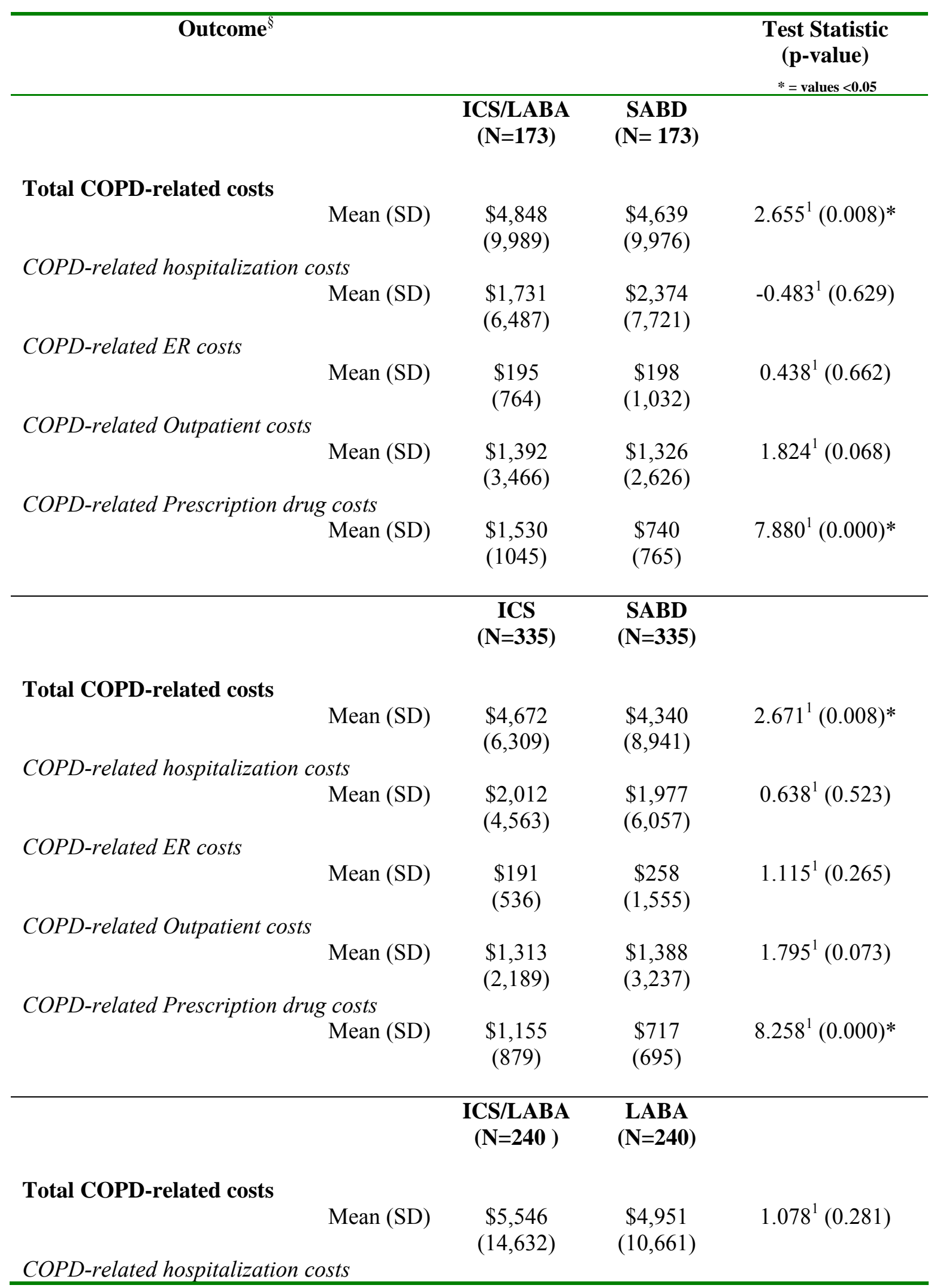




\begin{tabular}{lcccc}
\hline & Mean (SD) & $\begin{array}{c}\$ 2,763 \\
(11,688)\end{array}$ & $\begin{array}{c}\$ 1,889 \\
(6,255)\end{array}$ & $0.977^{1}(0.329)$ \\
COPD-related ER costs & & & & \\
COPD-related Outpatient costs & Mean (SD) & $\$ 129(483)$ & $\$ 254(890)$ & $-0.773^{1}(0.440)$ \\
& Mean (SD) & $\$ 1,259$ & $\$ 1,720$ & $-0.165^{1}(0.869)$ \\
COPD-related Prescription drug costs & $(3,499)$ & $(4,843)$ & \\
& Mean (SD) & $\$ 1,394$ & $\$ 1,088$ & $4.392^{1}(0.000)^{*}$ \\
& & $(986)$ & $(980)$ & \\
& & & & \\
\hline
\end{tabular}

${ }^{*} \mathrm{p}<0.05$

$\S$ Analysis of difference in outcome controlled for variables included in the propensity score model: age, gender, race, countysmokerate, state, D'Hoore-CCI, number of other chronic conditions, number of inhaled SABA canisters, number of inhaled IPR canisters, number of other doseforms of SABD, use of nebulized SABD, use of Hosp/ER for COPD or COPD-related comorbid disorders, \# of physician visits for COPD or COPD-related comorbid disorders, presence of asthma, and use of home oxygen therapy.

${ }^{1}$ Wilcoxon signed rank test statistic 


\section{Discussion for Research Objectives 5, 6, and 7}

Research objective 5 assessed the impact of ICS either alone or in combination with an inhaled long-acting beta-agonist on risk of a severe exacerbation in comparison to recipients with COPD using short-acting bronchodilators alone or using an inhaled long-acting betaagonist. The results showed that ICS either alone or in combination with an inhaled longacting beta-agonist did not reduce the risk of severe exacerbation.

Six randomized trials have reported the impact of ICS alone on exacerbations, but have not specifically differentiated between moderate and severe exacerbations. Of the six trials that reported ICS use and exacerbations, four did not show any significant difference (Weir et al., 1999) (Bourbeau et al., 1998) (Lung Health Study Group, 2000) (Vestbo et al., 1999) in reducing exacerbations. The lack of a significant difference in two of the four trials can be attributed to lower sample size ( $\mathrm{n}=79$ and $\mathrm{n}=98$ ) (Weir et al., 1999) (Bourbeau et al., 1998), and in the other two to the COPD severity level of the patients (Lung Health Study Group, 2000) (Vestbo et al., 1999). Specifically, the mean $\mathrm{FEV}_{1}$ of the patients in the latter two trials ranged from $68 \%$ to $85 \%$ of the predicted value. This is in contrast to the mean $\mathrm{FEV}_{1}$ of less than $50 \%$ of the predicted value in patients in the two trials that showed significant differences (Burge et al., 2000; Paggiaro et al., 1998). In the present study, data on $\mathrm{FEV}_{1}$ were not available, and hence it is not possible to determine the severity level based on $\mathrm{FEV}_{1}$. However, other measures of COPD severity were assessed at baseline such as use of home oxygen therapy, use of nebulized short-acting bronchodilators, and presence of hospitalization or ER visit for COPD or a COPD-related disorder. In the present study, home oxygen therapy was used by at least $15 \%$ of the cohort, nebulized short-acting bronchodilators were used by at least $20 \%$ of the cohort, and approximately $45 \%$ had at least one hospitalization or ER visit for COPD or a COPD-related comorbid disorder. Thus, the cohort in the present study was more likely to have moderate to severe COPD than mild COPD. However, within this group varying levels of severity can exist, and it was not possible to isolate a population with a higher severity level in which a beneficial effect of ICS may have been identified.

Observational studies have provided conflicting evidence regarding the impact of ICS on the risk of severe exacerbations. The impact of ICS on the risk of severe exacerbations in the present study is similar to the findings of two other observational studies. Fan et al. used a 
cohort study design, and found a risk ratio of 0.85 (95\% CI: 0.67 - 1.06) (Fan et al., 2003). Bourbeau et al. used a nested case-control study design, and found a risk ratio of 1.07 (95\% CI: $0.91-1.27$ ) (Bourbeau et al., 2003). However, Sin et al. and Soriano et al. have found ICS to reduce the risk of a severe exacerbation by 24 percent and 16 percent, respectively (Sin et al., 2001; Soriano et al., 2003). Similarly, a study using the Pharmetrics database showed that patients treated with ICS alone reduced the risk of a COPD-related hospitalization by 29 percent (Burney et al., 2003).

The discrepancy in the findings from observational studies has been considered by some researchers to be a result of creation of immortal time bias (Suissa, 2003). This reasoning has been further debated since studies that took specific measures to account for immortal time bias found a null effect of the impact of ICS on severe exacerbations (Fan et al., 2003; Bourbeau et al., 2003). Other studies where immortal time bias was found to exist were reanalyzed to eliminate this bias without any change in the study findings ( $\mathrm{Sin}, \mathrm{Man}, \& \mathrm{Tu}$, 2003b). In the present study, exposure status was defined based on the prescription received on the index date. There was no period of time where exposure status and outcome assessment were simultaneously determined, thus excluding the creation of immortal time bias.

Of two randomized trials that have assessed the effect of combination ICS/LABA on the risk of a severe exacerbation, one did not show any differences in the number of hospital admissions experienced by patients compared to a placebo group (Calverley et al., 2003a). In the other trial, a 24 percent reduction was found for severe exacerbations which was defined as the presence of hospitalizations or the requirement of oral steroids and/or antibiotics (Szafranski et al., 2003). Only three observational studies have specifically assessed the impact of combination ICS/LABA compared to the use of short-acting bronchodilators alone. One study was similar to the present study in that it used a similar cohort study design and Medicaid data from another state and found a risk reduction of 35 percent $(\mathrm{HR}=0.653 ; 95 \% \mathrm{CI}=0.43$ to 0.99$)$ (Rascati et al., 2005). The only difference between two studies was the definition of combination therapy, which in the present study referred to initiating on the combination product of ICS/LABA (Advair®), while in the Rascati et al. study, the receipt of concomitant prescriptions of ICS and LABA during a 60 day period was designated as combination therapy. 
Research objective 6 assessed the impact of ICS either alone or in combination with an inhaled long-acting beta-agonist on the frequency of moderate exacerbations in comparison to recipients with COPD using short-acting bronchodilators alone or using a long-acting betaagonist. In the present study, recipients initiated on ICS alone had a significantly higher number of moderate exacerbations ( 0.28 vs. 0.18 ; $\mathrm{p}=0.003)$. Only two observational studies have assessed the impact of ICS alone on the risk of a moderate exacerbation. Fan et al. found a null effect of $\mathrm{HR}=1.13(95 \% \mathrm{CI}=0.94-1.36)$ (Fan et al., 2003). On the other hand, a study by de Melo et al. using the Saskatechewan database found a significantly increased risk of exacerbations with an odds ratio of 1.27 (95\% CI: 1.08 to 1.48) (de Melo et al., 2004a). The authors attributed the increased risk to residual confounding due to a selection bias rather than to a detrimental effect of ICS. In the present study, propensity scores were used to account for the selection bias in those receiving ICS. However, propensity scores cannot account for unobserved factors (D'Agostino, Jr., 1998). It is well known that acute exacerbations of COPD have a heterogenous etiology and pathophysiology, and are the result of a variety of causes including bacterial and viral infections, cold weather, and interruption of regular treatment (Burge et al., 2003). Thus, it is possible that unobservable factors affecting exacerbations were not captured in the propensity score leading to residual confounding.

In one randomized trial, combination ICS/LABA (fluticasone/salmeterol) was shown to reduce the number and risk of exacerbations requiring oral corticosteroid courses (moderate exacerbations) compared to a placebo group (Calverley et al., 2003a). No observational studies have been conducted of the impact of ICS/LABA on the frequency of moderate exacerbations. In the present study, no difference was seen in the rate of moderate exacerbations between those initiated on combination ICS/LABA versus those on shortacting bronchodilators alone.

In the present study, the combination ICS/LABA (fluticasone/salmeterol) did not significantly reduce exacerbations compared to LABA (salmeterol) monotherapy. This finding is similar to a large randomized trial comparing combination ICS/LABA (fluticasone/salmeterol) to the LABA (salmeterol) component (Calverley et al., 2003a). No observational study has specifically compared the combination of ICS/LABA to the LABA component, but three observational studies found a significant risk reduction for combination 
ICS/LABA compared to an insignificant risk reduction for the LABA (salmeterol) monotherapy component in comparison to the reference short-acting bronchodilator group (Soriano et al., 2003; Burney et al., 2003; Rascati et al., 2005).

Research objective 7 compared direct costs between those initiated on ICS either alone or in combination with an inhaled long-acting beta-agonist compared to those using short-acting bronchodilators alone or an inhaled long-acting beta-agonist. The results of the economic evaluation mirrored the results of the objective 5 and 6 . Since no difference was seen in the risk of severe exacerbations and the frequency of moderate exacerbations, no differences were observed in the hospitalization, ER, or outpatient cost components. Those using ICS either alone or in combination incurred \$200 to \$300 higher COPD-related total costs compared to those using short-acting bronchodilators alone. This difference was primarily driven by differences in the prescription drug cost component. Two studies have conducted a cost-analysis of using ICS either alone or in combination with an inhaled long-acting betaagonist compared to using only short-acting bronchodilators. One study empirically determined costs in a clinical trial, and found those using ICS alone incurred lower total cost per day compared to those in a placebo group, but with no difference in the prescription drug cost component between groups (Ayres et al., 2003). The lower cost found among the ICS group was driven by a lower mean cost for hospitalizations and outpatient visits, since the ICS group was found to have a reduced risk of moderate to severe exacerbations. Another study using claims data from an HMO had similar findings to the present study (Gagnon et al., 2005). Patients using ICS either alone or in combination with an inhaled long-acting beta-agonist incurred higher costs during a 3 year period than those using short-acting bronchodilators. Also, patients using combination ICS/LABA incurred higher costs compared to those using LABA monotherapy. Statistical significance tests were not computed, and differences in cost components were not identified. 


\section{Phase III}

Phase III assesses the impact of ICS on mortality using two different study designs.

Objective 8 uses a case-control study design and Objective 9 uses a historical cohort study design to ascertain this association.

TABLE 39: Sample extraction for Phase III - Objective 8

\begin{tabular}{|c|c|c|}
\hline Step & Outcome (WV) & Outcome (KY) \\
\hline $\begin{array}{l}\text { 1. \# 35-64 years of age with at least one } \\
\text { medical claim with a primary diagnosis of } \\
\text { COPD between WV: Jul 1, } 1997 \text { to Jun 30, } \\
2003 \text { and KY: Jan 1, } 1998 \text { to Dec } 31,2003 \text {. }\end{array}$ & $A=20,193$ & $A=27,901$ \\
\hline $\begin{array}{l}\text { 2. \# of A who died between WV: Jul 1, } 1998 \\
\text { and Jun 30, } 2003 \text { and KY: Jan 1, } 1999 \text { and } \\
\text { Dec 31, 2003 }\end{array}$ & $\mathrm{B}=1,942$ & $\mathrm{~B}=2,728$ \\
\hline 3. \# of controls available for matching to cases & $\mathrm{C}=\mathrm{A}-\mathrm{B}=18,251$ & $\mathrm{C}=\mathrm{A}-\mathrm{B}=25,173$ \\
\hline $\begin{array}{l}\text { 4. \# of } \mathrm{B}+\mathrm{C} \text { continuously eligible for } \\
\text { Medicaid and not enrolled in managed care } \\
\text { at anytime } 1 \text { year before index date. }\end{array}$ & $\mathrm{D}=2,563$ & $\mathrm{D}=3,773$ \\
\hline $\begin{array}{l}\text { 5. \# of D with at least one hospitalization or } \\
\text { ER visit or at least two outpatient claims on } \\
\text { different dates with a primary diagnosis of } \\
\text { COPD in } 1 \text { year before index date }\end{array}$ & $\begin{array}{l}E=1,277 \\
- \text { Cases }=910 \\
- \text { Controls }=367\end{array}$ & $\begin{array}{l}E=1,905 \\
- \text { Cases }=1,285 \\
- \text { Controls }=620\end{array}$ \\
\hline $\begin{array}{l}\text { 6. \# of } E \text { without diagnosis of cystic fibrosis, } \\
\text { fibrosis due to tuberculosis, respiratory } \\
\text { cancer, and bronchiectasis during study } \\
\text { period }\end{array}$ & $\begin{array}{l}F=1,042 \\
- \text { Cases }=691 \\
- \text { Controls }=351\end{array}$ & $\begin{array}{l}F=1,529 \\
- \text { Cases }=927 \\
\text {-Controls }=602\end{array}$ \\
\hline 7. \# of matched pairs & $\begin{array}{l}\mathrm{G}=101 \\
- \text { Cases }=101 \\
- \text { Controls }=101\end{array}$ & $\begin{array}{l}\mathrm{G}=175 \\
- \text { Cases }=175 \\
- \text { Controls }=175\end{array}$ \\
\hline 8. Total sample size & $\begin{array}{l}\mathrm{H}=552 \\
- \text { Cases }=276 \\
- \text { Controls }=276\end{array}$ & \\
\hline
\end{tabular}


Table 39 shows that there were more cases than controls that met the study inclusion criteria for objective 8. Accordingly, a 1:1 match was done by age, gender, and race to obtain a final sample size of 276 matched pairs. The average age was 51 years with a majority of women (61\%) and whites (95\%). Table 40 outlines overall comorbidity and COPD severity of the cases and controls in the year before the index date. As expected, cases (those who died) were more likely to have been sicker with a mean D'Hoore Charlson comorbidity index of 4.6 versus 1.7 for controls. Additionally, cases had greater COPD severity as measured by the proportion using oral corticosteroids (55.7\% vs. $48.9 \%)$, nebulized short-acting bronchodilators (59.8\% vs. 38.4\%), and home oxygen therapy (44.2\% vs. $28.3 \%$ ). Of note, cases were significantly more likely to have a hospitalization or ER visit (82.6\% vs. $49.6 \%$ ), and significantly less likely to have a physician visit for COPD or COPD-related comorbid disorder (73.2\% vs. 85.6\%) compared to controls. Although cases were more likely to have asthma, this difference was not statistically significant $(20.7 \%$ vs. $15.5 \%)$. 
TABLE 40: Characteristics of study sample by case-control status in pre-index period (Phase IIIObjective 8)

\begin{tabular}{|c|c|c|c|}
\hline Characteristic & $\begin{array}{c}\text { Cases } \\
(\mathrm{N}=276)\end{array}$ & $\begin{array}{l}\text { Controls } \\
(N=276)\end{array}$ & $\begin{array}{l}\text { Test Statistic } \\
\text { (p-value) }\end{array}$ \\
\hline \multicolumn{4}{|l|}{ Demographic } \\
\hline Age, mean years (SD) & \multicolumn{2}{|c|}{$51(0.34)$} & NA \\
\hline Female, \% (n) & \multicolumn{2}{|c|}{$61.0 \%(167)$} & NA \\
\hline White, \% (n) & \multicolumn{2}{|c|}{$95.1 \%(234)$} & NA \\
\hline Countysmokerate $>=31 \%$ & $53.6 \%(148)$ & $62.0 \%(171)$ & $4.940^{1}(0.033)^{*}$ \\
\hline \multicolumn{4}{|l|}{ Overall Comorbidity } \\
\hline \multicolumn{4}{|l|}{ D'Hoore-CCI ${ }^{\mathrm{a}}$} \\
\hline $\begin{array}{r}\text { Mean (SD) } \\
\text { Median }\end{array}$ & $\begin{array}{l}4.6(3.8) \\
4.0\end{array}$ & $\begin{array}{c}1.7(2.0) \\
1.0\end{array}$ & $-9.386^{2}(0.000)^{*}$ \\
\hline $\begin{array}{r}\text { Number of other chronic conditions }{ }^{\mathrm{b}} \\
\text { Mean (SD) } \\
\text { Median }\end{array}$ & $\begin{array}{l}2.2(1.5) \\
2.0\end{array}$ & $\begin{array}{l}2.2(1.4) \\
2.0\end{array}$ & $-0.809^{2}(0.419)$ \\
\hline \multicolumn{4}{|l|}{ COPD severity } \\
\hline $\begin{array}{r}\text { Use of inhaled SABA c canisters, } \%(\mathrm{n}) \\
\text { Mean \# of canisters (SD) } \\
\text { Median }\end{array}$ & $\begin{array}{c}47.4 \%(131) \\
4.3(8.9) \\
0.0\end{array}$ & $\begin{array}{c}55.0 \%(152) \\
3.6(6.0) \\
1.0\end{array}$ & $\begin{array}{l}3.270^{1}(0.085) \\
-0.057^{2}(0.954)\end{array}$ \\
\hline $\begin{array}{r}\text { Use of inhaled IPR canisters, } \%(\mathrm{n}) \\
\text { Mean \# of canisters (SD) } \\
\text { Median }\end{array}$ & $\begin{array}{c}46.4 \%(128) \\
3.6(5.9) \\
0.0\end{array}$ & $\begin{array}{c}48.9 \%(135) \\
3.0(4.4) \\
0.0\end{array}$ & $\begin{array}{l}0.350^{1}(0.556) \\
-0.368^{2}(0.713)\end{array}$ \\
\hline $\begin{array}{r}\text { Use of other doseform } \mathrm{SABD}^{\mathrm{c}}, \%(\mathrm{n}) \\
\text { Mean \# of Rx (SD) } \\
\text { Median }\end{array}$ & $\begin{array}{c}37.3 \%(103) \\
3.0(5.1) \\
0.0\end{array}$ & $\begin{array}{c}39.1 \%(173) \\
3.2(5.1) \\
0.0\end{array}$ & $\begin{array}{l}0.180^{1}(0.736) \\
-0.547^{2}(0.584)\end{array}$ \\
\hline $\begin{array}{l}\text { Use of OCS }, \%(n) \\
\text { Mean \# of Rx (SD) } \\
\text { Median }\end{array}$ & $\begin{array}{c}55.7 \%(154) \\
3.0(4.4) \\
1.0\end{array}$ & $\begin{array}{c}48.9 \%(135) \\
1.4(2.5) \\
0.0\end{array}$ & $\begin{array}{c}2.600^{1}(0.127) \\
-4.004^{2}(0.000)^{*}\end{array}$ \\
\hline $\begin{array}{r}\text { Use of nebulized SABD }{ }^{\mathrm{c}}, \%(\mathrm{n}) \\
\text { Mean \# of Rx (SD) } \\
\text { Median }\end{array}$ & $\begin{array}{c}59.8 \%(165) \\
6.0(9.6) \\
2.0\end{array}$ & $\begin{array}{c}38.4 \%(106) \\
2.5(5.2) \\
0.0\end{array}$ & $\begin{array}{l}27.410^{1}(0.000)^{*} \\
-5.556^{2}(0.000)^{*}\end{array}$ \\
\hline $\begin{array}{l}\text { Use of Hosp/ER for COPD or COPD- } \\
\text { related comorbid disorders }{ }^{\text {e }} \% \text { (n) }\end{array}$ & $82.6 \%(228)$ & $49.6 \%(137)$ & $58.730^{1}(0.000)^{*}$ \\
\hline
\end{tabular}




\begin{tabular}{|c|c|c|c|}
\hline $\begin{array}{r}\text { Mean (SD) } \\
\text { Median }\end{array}$ & $\begin{array}{l}4.0(4.1) \\
3.0\end{array}$ & $\begin{array}{c}1.3(3.2) \\
0.0\end{array}$ & $-9.785^{2}(0.000)^{*}$ \\
\hline $\begin{array}{l}\text { Use of Physician visits for COPD or } \\
\text { COPD-related comorbid disorders }{ }^{\mathrm{e}}, \% \\
\text { (n) }\end{array}$ & $73.2 \%(202)$ & $85.6 \%(236)$ & $12.570^{1}(0.000)^{*}$ \\
\hline $\begin{array}{r}\text { Mean (SD) } \\
\text { Median }\end{array}$ & $\begin{array}{l}3.4(4.8) \\
2.0\end{array}$ & $\begin{array}{c}4.6(4.1) \\
4.0\end{array}$ & $4.396^{2}(0.000)^{*}$ \\
\hline Presence of asthma, \% (n) & $20.7 \%(57)$ & $15.5 \%(43)$ & $3.060^{1}(0.103)$ \\
\hline Use of Home oxygen therapy & $44.2 \%(122)$ & $28.3 \%(78)$ & $15.370^{2}(0.000)^{*}$ \\
\hline $\begin{array}{l}{ }^{*} \mathrm{p}<0.05 \\
{ }^{\mathrm{a}} \text { Excludes asthma } \\
\mathrm{b} \text { Includes hypercholesterolemia, hypertension } \\
\text { gall bladder disease, depression and anxiety, a } \\
{ }^{\mathrm{c}} \text { Short-acting beta-agonist: includes levalbute } \\
{ }^{\mathrm{d}} \text { Short-acting bronchodilators: includes levall } \\
\text { theophylline, and ipratropium. } \\
{ }^{\mathrm{d}} \text { Oral corticosteroids: includes prednisone, pr } \\
\text { dexamethasone, betamethasone, oral triamcin } \\
{ }^{\mathrm{e}} \text { Includes COPD, asthma, upper respiratory tr } \\
\text { allergic rhinitis, other diseases of the lung, co } \\
\text { base imbalance. } \\
{ }^{1} \text { McNemar test statistic: }{ }^{2} \text { Wilcoxon signed ran }\end{array}$ & $\begin{array}{l}\text { ther heart diseas } \\
\text { schizophrenia. } \\
\text { albuterol, meta } \\
\text { erol, albuterol, n } \\
\text { nisolone, methyl } \\
\text { ne, oral budeson } \\
\text { infections, lowe } \\
\text { estive heart failu } \\
\text { est statistic }\end{array}$ & $\begin{array}{l}\text { IDS, rheumatc } \\
\text { erenol, bitolter } \\
\text { proterenol, bitc } \\
\text { Inisolone, corti } \\
\text { spiratory tract } \\
\text { ind disorders o }\end{array}$ & $\begin{array}{l}\text { Irthritis, osteoarthritis, } \\
\text { erbutaline. } \\
\text { ol, terbutaline, } \\
\text { e, hydrocortisone, } \\
\text { ctions, septicemia, } \\
\text { id, electrolyte, and acid- }\end{array}$ \\
\hline
\end{tabular}

\section{Research Objective 8: To compare the odds of exposure to inhaled corticosteroids between those who died and those who did not die during the study period (case-control study design).}

A lower proportion of cases were found to have at least two prescriptions of inhaled corticosteroids in the pre-index period compared to controls $(39.1 \%$ vs. $46.0 \%)$ with a crude odds ratio of 0.768 (0.544-1.080) (Table 41). In adjusted analyses (Table 42) controlling for all possible confounders, recipients exposed to inhaled corticosteroids had a 40 percent lower probability of death compared to those who did not use inhaled corticosteroids $(\mathrm{OR}=0.605)$. However, this effect was not significant. A power analysis was conducted using Dupont's methodology for calculating power in matched case-control designs (Dupont, 1988). This method computes power or sample size based on the alternative odds ratio, the correlation of exposure between pairs in the case-control set, and the probability of exposure in the controls. Based on the study inputs, the analysis computed a power of $46 \%$. 
TABLE 41: Exposure to inhaled corticosteroids in pre-index period by case-control status (Phase III - Objective 8)

\begin{tabular}{lcccc}
\hline & $\begin{array}{c}\text { Cases } \\
(\mathbf{N}=\mathbf{2 7 6})\end{array}$ & $\begin{array}{c}\text { Controls } \\
(\mathbf{N}=276)\end{array}$ & $\begin{array}{c}\text { Crude Odds } \\
\text { Ratio (95\% CI) }\end{array}$ & $\begin{array}{c}\text { Test Statistic } \\
\text { (p-value) }\end{array}$ \\
\hline Exposure to ICS (\%, n) & $\begin{array}{c}39.1 \% \\
(108)\end{array}$ & $\begin{array}{c}46.0 \% \\
(127)\end{array}$ & $\begin{array}{c}0.768 \\
(0.544-1.080)\end{array}$ & $2.49(0.135)$ \\
\hline
\end{tabular}

TABLE 42: Conditional logistic regression of the impact of inhaled corticosteroids on mortality (Phase III - Objective 8)

DV: Probability of death

\begin{tabular}{|c|c|c|c|}
\hline Variable & Odds ratio $(S E)$ & $95 \% C I$ & $\begin{array}{c}p \\
\text { value }\end{array}$ \\
\hline \multicolumn{4}{|l|}{ Exposure to ICS (Ref: No) } \\
\hline 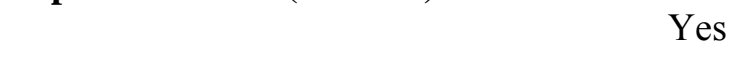 & $0.605(0.179)$ & $0.338-1.080$ & 0.089 \\
\hline \multicolumn{4}{|l|}{ Demographic } \\
\hline \multicolumn{4}{|l|}{ County_Smokerate(Ref: $<31 \%)$} \\
\hline$>=31 \%$ & $0.702(0.216)$ & $0.385-1.281$ & 0.249 \\
\hline \multicolumn{4}{|l|}{ Overall comorbidity } \\
\hline D’Hoore-CCI & $1.465(0.096)$ & $1.288-1.666$ & $0.000 *$ \\
\hline Number of other chronic conditions & $0.719(0.078)$ & $0.580-0.889$ & $0.002 *$ \\
\hline \multicolumn{4}{|l|}{ COPD severity } \\
\hline \# of inhaled SABA canisters & $1.014(0.016)$ & $0.982-1.048$ & 0.398 \\
\hline \# of inhaled IPR canisters & $1.063(0.029)$ & $1.007-1.122$ & $0.026^{*}$ \\
\hline \# of other SABD prescriptions & $1.011(0.027)$ & $0.959-1.065$ & 0.693 \\
\hline \# of OCS prescriptions & $1.107(0.049)$ & $1.015-1.208$ & $0.022 *$ \\
\hline Use of nebulized SABD & $1.619(0.469)$ & $0.920-2.857$ & 0.096 \\
\hline $\begin{array}{r}\text { \# of Hosp/ER visits for COPD or COPD- } \\
\text { related comorbid disorders }\end{array}$ & $3.160(0.976)$ & $1.725-5.787$ & $0.000 *$ \\
\hline $\begin{array}{r}\text { \# of Physician visits for COPD or COPD- } \\
\text { related comorbid disorders }\end{array}$ & $0.302(0.103)$ & $0.154-0.591$ & $0.000^{*}$ \\
\hline Presence of asthma & $0.632(0.261)$ & $0.282-1.421$ & 0.267 \\
\hline Use of Home oxygen therapy & $1.740(0.530)$ & $0.957-3.163$ & 0.069 \\
\hline
\end{tabular}

$* \mathrm{p}<0.05$

Log likelihood $=-98.34 ; \chi^{2}=185.93 ; \mathrm{p}=0.000 *$

Pseudo R ${ }^{2}: 48.6 \%$ 
TABLE 43: Sample extraction for Phase III - Objective 9

\begin{tabular}{|c|c|c|c|}
\hline \multicolumn{2}{|r|}{ Step } & Outcome WV & \multirow{2}{*}{$\begin{array}{l}\text { Outcome KY } \\
\mathrm{A}=101,967 \\
\mathrm{ICS}=77,345 \\
\text { no } \mathrm{ICS}=246,222\end{array}$} \\
\hline 1. & $\begin{array}{l}\text { \# with a Rx claim for combination product } \\
\text { of ICS/LABA or ICS, or other COPD- } \\
\text { related drugs during: WV- Jul 1, } 1998 \text { to } \\
\text { Mar 31, } 2002 \text { and KY-Jan 1, } 1999 \text { to Sep } \\
30,2002\end{array}$ & $\begin{array}{l}\mathrm{A}=159,514 \\
\mathrm{ICS}=32,948 \\
\text { no } \mathrm{ICS}=126,566\end{array}$ & \\
\hline \multicolumn{4}{|c|}{$\begin{array}{l}\text { The date of first chronologically occurring prescription claim was defined as an index date fo } \\
\text { each person. The drug therapy groups represent number with respective prescription claim at } \\
\text { index date. }\end{array}$} \\
\hline 3. & $\begin{array}{l}\text { \# of A between } 35-64 \text { years, continuously } \\
\text { eligible for Medicaid and not enrolled in } \\
\text { managed care at anytime } 1 \text { year before } \\
\text { index date }\end{array}$ & $\begin{array}{l}\mathrm{B}=33,010 \\
\mathrm{ICS}=10,058 \\
\text { no } \mathrm{ICS}=22,952\end{array}$ & $\begin{array}{l}\mathrm{B}=62,408 \\
\mathrm{ICS}=19,810 \\
\text { no } \mathrm{ICS}=42,598\end{array}$ \\
\hline 4. & $\begin{array}{l}\text { \# of B with prescription drug patterns } \\
\text { specified in study criteria }\end{array}$ & $\begin{array}{l}\mathrm{C}=16,576 \\
\mathrm{ICS}=4,797 \\
\text { no } \mathrm{ICS}=11,779\end{array}$ & $\begin{array}{l}\mathrm{C}=30,937 \\
\mathrm{ICS}=9,152 \\
\text { no } \mathrm{ICS}=21,785\end{array}$ \\
\hline 5. & $\begin{array}{l}\text { \# of } \mathrm{C} \text { without diagnosis of cystic fibrosis, } \\
\text { fibrosis due to tuberculosis, respiratory } \\
\text { cancer, and bronchiectasis during study } \\
\text { period }\end{array}$ & $\begin{array}{l}\mathrm{D}=16,103 \\
\mathrm{ICS}=4,622 \\
\text { no } \mathrm{ICS}=11,481\end{array}$ & $\begin{array}{l}\mathrm{D}=30,219 \\
\mathrm{ICS}=8,909 \\
\text { no } \mathrm{ICS}=21,310\end{array}$ \\
\hline 6. & $\begin{array}{l}\text { \# of D with at least one hospitalization or } \\
\text { ER visit or at least two outpatient claims on } \\
\text { different dates with a primary or secondary } \\
\text { diagnosis of COPD }\end{array}$ & $\begin{array}{l}\mathrm{E}=3,237 \\
\mathrm{ICS}=1,534 \\
\text { no } \mathrm{ICS}=1,703\end{array}$ & $\begin{array}{l}\mathrm{E}=5,808 \\
\mathrm{ICS}=2,610 \\
\text { no } \mathrm{ICS}=3,198\end{array}$ \\
\hline & $\begin{array}{l}\text { \# of } E \text { who did not die during } 30 \text { days } \\
\text { following index date }\end{array}$ & $\begin{array}{l}\mathrm{F}=3,220 \\
\mathrm{ICS}=1,532 \\
\text { no } \mathrm{ICS}=1,688\end{array}$ & $\begin{array}{l}\mathrm{F}=5,790 \\
\mathrm{ICS}=2,602 \\
\text { no } \mathrm{ICS}=3,188\end{array}$ \\
\hline 8. & $\begin{array}{l}\text { \# of F continuously eligible for Medicaid } \\
\text { and not enrolled in managed care at anytime } \\
\text { till date of death for those who died or till } \\
\text { end of follow-up for those who did not die }\end{array}$ & $\begin{array}{l}\mathrm{G}=2,928 \\
\mathrm{ICS}=1,420 \\
\text { no } \mathrm{ICS}=1,508\end{array}$ & $\begin{array}{l}\mathrm{G}=5,341 \\
\mathrm{ICS}=2,420 \\
\text { no } \mathrm{ICS}=2,921\end{array}$ \\
\hline & $\begin{array}{l}\text { 1. \# of } \mathrm{G} \text { who had at least one additional } \\
\text { prescription claim of index drug therapy } \\
\text { in follow-up period. }\end{array}$ & $\begin{array}{l}\mathrm{H}=1,209 \\
\mathrm{ICS}=901 \\
\text { no } \mathrm{ICS}=308\end{array}$ & $\begin{array}{l}\mathrm{H}=2,308 \\
\mathrm{ICS}=1,696 \\
\text { no } \mathrm{ICS}=616\end{array}$ \\
\hline & 2. Final study sample size & $\begin{array}{l}\mathrm{J}=3,517 \\
\mathrm{ICS}=2,594 \\
\text { no } \mathrm{ICS}=923\end{array}$ & \\
\hline
\end{tabular}


A total of 3,517 recipients met the inclusion criteria for objective 9 of Phase III, the majority of which were prescribed an inhaled corticosteroid (ICS) (Table 43). Table 44 outlines the baseline characteristics of the study sample. Recipients who were prescribed inhaled corticosteroids were more likely to be younger females. Although the D'Hoore Charlson comorbidity index showed no differences, recipients in the ICS group had a higher mean number of other chronic conditions (2.0 vs. 1.8). Recipients with ICS were also more likely to have used oral corticosteroids ( $41.9 \%$ vs. $35.4 \%)$ and home oxygen therapy (18.1\% vs. $14.6 \%$ ) in the pre-index period, and have a hospitalization or ER visit for COPD or a COPD-related disorder in the pre-index period compared to those not prescribed an ICS (53.8\% vs. $46.8 \%)$. As expected, recipients with concomitant asthma were also more likely to have been prescribed an ICS ( $22.1 \%$ vs. $14.8 \%)$. Recipients who were not prescribed an ICS had a higher number of canisters of SABA and IPR, and a higher number of prescriptions of other dose forms of short-acting bronchodilators. However, this increased use may be a reflection of the study design, since those in the non-ICS group having a shortacting bronchodilator as the index prescription were required to have at least two prescriptions for a short-acting bronchodilator in the pre-index period of which one was for ipratropium. This was done in order to avoid including a relatively mild COPD population who use short-acting bronchodilators on an acute as-needed basis. 
TABLE 44: Baseline characteristics of study sample by drug therapy status (ICS vs. no ICS: Phase III-Objective 9)

\begin{tabular}{|c|c|c|c|}
\hline Characteristic & $\begin{array}{c}\text { ICS } \\
(\mathrm{N}=2,594)\end{array}$ & $\begin{array}{l}\text { No ICS } \\
(\mathrm{N}=923)\end{array}$ & $\begin{array}{l}\text { Test Statistic } \\
\text { (p-value) }\end{array}$ \\
\hline \multicolumn{4}{|l|}{ Demographic } \\
\hline Age, mean years (SD) & $51(7.6)$ & $53(7.4)$ & $4.289^{1}(0.000)^{*}$ \\
\hline Female, $\%(n)$ & $64.9 \%(1,675)$ & $58.3 \%(537)$ & $12.576^{2}(0.000)^{*}$ \\
\hline White, $\%(n)$ & $96.4 \%(2,246)$ & $97.7 \%(818)$ & $3.489^{2}(0.062)$ \\
\hline \multicolumn{4}{|l|}{ State, $\%(n)$} \\
\hline$W V$ & $34.7 \%(901)$ & $33.4 \%(308)$ & $0.562^{2}(0.453)$ \\
\hline$K Y$ & $65.3 \%(1,693)$ & $66.6 \%(615)$ & \\
\hline Countysmokerate $>=31 \%$ & $63.4 \%(1,644)$ & $67.2 \%(620)$ & $4.276^{2}(0.039)^{*}$ \\
\hline \multicolumn{4}{|l|}{ Overall Comorbidity } \\
\hline \multicolumn{4}{|l|}{$\mathrm{D}^{\prime}$ Hoore-CCI $\mathrm{a}^{\mathrm{a}}$} \\
\hline $\begin{array}{r}\text { Mean (SD) } \\
\text { Median }\end{array}$ & $\begin{array}{l}1.5(1.8) \\
1.0\end{array}$ & $\begin{array}{c}1.5(2.0) \\
1.0\end{array}$ & $-1.092^{3}(0.275)$ \\
\hline \multicolumn{4}{|l|}{ Number of other chronic conditions ${ }^{b}$} \\
\hline $\begin{array}{r}\text { Mean (SD) } \\
\text { Median }\end{array}$ & $\begin{array}{l}2.0(1.3) \\
2.0\end{array}$ & $\begin{array}{c}1.8(1.3) \\
2.0\end{array}$ & $-4.377^{3}(0.000)^{*}$ \\
\hline \multicolumn{4}{|l|}{ COPD severity } \\
\hline $\begin{array}{r}\text { Use of inhaled SABA }{ }^{\mathrm{c}} \text { canisters, } \%(\mathrm{n}) \\
\text { Mean \# of canisters (SD) } \\
\text { Median }\end{array}$ & $\begin{array}{c}59.3 \%(1,538) \\
4.1(7.2) \\
1.0\end{array}$ & $\begin{array}{c}63.8 \%(589) \\
4.8(7.8) \\
2.0\end{array}$ & $\begin{array}{l}5.827^{2}(0.016)^{*} \\
2.818^{3}(0.005)^{*}\end{array}$ \\
\hline $\begin{array}{r}\text { Use of inhaled IPR canisters, \% (n) } \\
\text { Mean \# of canisters (SD) } \\
\text { Median }\end{array}$ & $\begin{array}{l}43.9 \%(1,138) \\
2.6(5.5) \\
0.0\end{array}$ & $\begin{array}{c}67.4 \%(622) \\
4.3(6.5) \\
2.0\end{array}$ & $\begin{array}{c}150.618^{2}(0.000)^{*} \\
12.198^{3}(0.000)^{*}\end{array}$ \\
\hline $\begin{array}{r}\text { Use of other doseform } \mathrm{SABD}^{\mathrm{c}}, \%(\mathrm{n}) \\
\text { Mean \# of Rx (SD) } \\
\text { Median }\end{array}$ & $\begin{array}{c}31.7 \%(821) \\
2.3(4.4) \\
0.0\end{array}$ & $\begin{array}{c}40.3 \%(372) \\
2.9(4.8) \\
0.0\end{array}$ & $\begin{array}{c}22.743^{2}(0.000)^{*} \\
4.833^{3}(0.000)^{*}\end{array}$ \\
\hline $\begin{array}{r}\text { Mean \# of Rx (SD) } \\
\text { Median }\end{array}$ & $\begin{array}{c}41.9 \%(1,087) \\
1.2(2.3) \\
0.0\end{array}$ & $\begin{array}{c}35.4 \%(327) \\
1.1(2.6) \\
0.0\end{array}$ & $\begin{array}{l}11.878^{1}(0.001)^{*} \\
-3.427^{3}(0.001)^{*}\end{array}$ \\
\hline Use of nebulized SABD ${ }^{\mathrm{c}}, \%(\mathrm{n})$ & $27.5 \%(713)$ & $25.7 \%(237)$ & $1.130^{1}(0.288)$ \\
\hline
\end{tabular}




\begin{tabular}{|c|c|c|c|}
\hline $\begin{array}{r}\text { Mean \# of Rx (SD) } \\
\text { Median }\end{array}$ & $\begin{array}{c}1.8(4.8) \\
0.0\end{array}$ & $\begin{array}{c}2.1(5.7) \\
0.0\end{array}$ & $-0.527^{3}(0.580)$ \\
\hline $\begin{array}{l}\text { Use of Hosp/ER for COPD or COPD- } \\
\text { related comorbid disorders }{ }^{\mathrm{e}}, \%(\mathrm{n})\end{array}$ & $53.8 \%(1,398)$ & $46.8 \%(432)$ & $13.679^{1}(0.000)^{*}$ \\
\hline Mean (SD) & $0.5(0.5)$ & $0.5(0.5)$ & $-3.698^{3}(0.000)^{*}$ \\
\hline Median & 1.0 & 0.0 & \\
\hline $\begin{array}{l}\text { Use of Physician visits for COPD or } \\
\text { COPD-related comorbid disorders }{ }^{\mathrm{e}}, \% \text { (n) }\end{array}$ & $85.1 \%(2,210)$ & $84.2 \%(778)$ & $0.429^{1}(0.513)$ \\
\hline Mean (SD) & $0.9(0.4)$ & $0.8(0.4)$ & $-0.655^{3}(0.513)$ \\
\hline Median & 1.0 & 1.0 & \\
\hline Presence of asthma, $\%$ (n) & $22.1 \%(572)$ & $14.8 \%(137)$ & $21.975^{1}(0.000)^{*}$ \\
\hline Use of Home oxygen therapy, \%(n) & $18.1 \%(167)$ & $14.6 \%(378)$ & $6.445^{1}(0.011)^{*}$ \\
\hline
\end{tabular}

*p $<0.05$

${ }^{\mathrm{a}}$ Excludes asthma

${ }^{\mathrm{b}}$ Includes hypercholesterolemia, hypertension, other heart disease, AIDS, rheumatoid arthritis, osteoarthritis, gall bladder disease, depression and anxiety, and schizophrenia.

${ }^{c}$ Short-acting beta-agonist: includes levalbuterol, albuterol, metaproterenol, bitolterol, terbutaline.

${ }^{\mathrm{d}}$ Short-acting bronchodilators: includes levalbuterol, albuterol, metaproterenol, bitolterol, terbutaline, theophylline, and ipratropium.

${ }^{\mathrm{d}}$ Oral corticosteroids: includes prednisone, prednisolone, methylprednisolone, cortisone, hydrocortisone, dexamethasone, betamethasone, oral triamcinolone, oral budesonide.

${ }^{\mathrm{e}}$ Includes COPD, asthma, upper respiratory tract infections, lower respiratory tract infections, septicemia, allergic rhinitis, other diseases of the lung, congestive heart failure, and disorders of fluid, electrolyte, and acidbase imbalance.

${ }^{1}$ t statistic; ${ }^{2}$ Chi-square test statistic; ${ }^{3}$ Mann-Whitney U-statistic

\section{Research Objective 9: To conduct a mediation analysis to examine the relationship between use of ICS, severe exacerbation and death (historical cohort study design).}

Recipients who were prescribed an inhaled corticosteroid were significantly less likely to have died in the follow-up period compared to recipients who were not prescribed and inhaled corticosteroid (Table 40: $2.0 \%$ vs. $3.7 \%$, $\mathrm{p}=0.005$ ). Propensity scores were used to account for this selection bias by incorporating it in the multivariate analyses as a covariate. The logistic regression model used to obtain propensity scores in provided in Table 46. Quintiles of the propensity score were created (Table 47), and differences in baseline characteristics between the therapy groups were analyzed within each quintile using two-way ANOVAs for continuous variables and Cochran-Mantel Haenzel statistics for categorical variables. Table 48 shows that there were no significant differences in baseline 
characteristics within each propensity score quintile. Hence the propensity score quintile was used in the multivariate models. The survival advantage persisted for recipients being prescribed an inhaled corticosteroid even after controlling for the propensity score quintile and the recipient's state (Table 49, Figure 19). Recipients prescribed an inhaled corticosteroid were $\sim 48$ percent less likely to die in the follow-up period compared to those who were not prescribed an inhaled corticosteroid. The log-rank test of time to death had a power of $95.2 \%$ (Bauer D \& Lavery R, 2004). The absolute risk reduction was

Objective 9 also wanted to assess if any survival advantage afforded by using inhaled corticosteroids was mediated by the effect of inhaled corticosteroids on severe exacerbations. Hence, the impact of inhaled corticosteroids on risk of a severe exacerbation in the follow-up period was assessed. In univariate analyses, a slightly higher but insignificant proportion of recipients prescribed an inhaled corticosteroid had a severe exacerbation compared to recipients not being prescribed an inhaled corticosteroid (Table 45: 16.7\% vs. 14.2\%; $\mathrm{p}=0.08$ ). However, since this effect might be due to a selection bias, propensity scores were used. Table 50 and Figure 20 shows that after controlling for propensity score quintile and state, there was no effect of inhaled corticosteroids on severe exacerbations in the follow-up period $(H R=1.186 ; p=0.112)$. Since no effect was found, a mediation analysis was not conducted. The log-rank test of time to death had a power of 58.0\% (Bauer D et al., 2004) 
TABLE 45: Risk of mortality and severe exacerbation in follow-up period by drug therapy status (ICS vs. no ICS: Phase III - Objective 9)

\begin{tabular}{|c|c|c|c|}
\hline Characteristic & $\begin{array}{c}\text { ICS } \\
(\mathrm{N}=2,594)\end{array}$ & $\begin{array}{l}\text { No ICS } \\
(\mathrm{N}=923)\end{array}$ & $\begin{array}{c}\text { Test Statistic } \\
\text { (p-value) }\end{array}$ \\
\hline Died $(\%, n)$ & $2.0 \%(52)$ & $3.7 \%(34)$ & $8.045^{1}(0.005)^{*}$ \\
\hline \multicolumn{4}{|l|}{ Time to death (days) } \\
\hline Mean (SE) & $363(0.5)$ & $359(1.3)$ & $8.586^{2}(0.003)^{*}$ \\
\hline Severe exacerbations $(\%, n)$ & $16.7 \%(432)$ & $14.2 \%(131)$ & $3.067^{1}(0.080)$ \\
\hline \multicolumn{4}{|l|}{ Time to severe exacerbation (days) } \\
\hline Mean (SE) & $335(2.8)$ & $327(1.8)$ & $3.220^{2}(0.073)$ \\
\hline
\end{tabular}

*p $<0.05$

${ }^{1}$ Chi-square test statistic; ${ }^{2}$ Log-rank test statistic 
TABLE 46: Logistic regression predicting the probability of being prescribed an inhaled corticosteroid

\begin{tabular}{|c|c|c|c|}
\hline \multirow[b]{2}{*}{ Variable } & \multicolumn{2}{|c|}{ DV: Probability of receiving ICS } & \multirow[b]{2}{*}{$\begin{array}{c}p \\
\text { value }\end{array}$} \\
\hline & Odds ratio (SE) & $95 \% C I$ & \\
\hline \multicolumn{4}{|l|}{ Demographic } \\
\hline Age (years) & $0.985(0.006)$ & $0.975-0.997$ & $0.010 *$ \\
\hline \multicolumn{4}{|l|}{ Gender (Ref: Male) } \\
\hline Female & $1.164(0.100)$ & $0.984-1.377$ & 0.076 \\
\hline \multicolumn{4}{|l|}{ Race (Ref: non-White) } \\
\hline White & $0.695(0.182)$ & $0.415-1.163$ & 0.166 \\
\hline \multicolumn{4}{|l|}{ State (Ref: WV) } \\
\hline $\mathrm{KY}$ & $0.930(0.099)$ & $0.755-1.146$ & 0.498 \\
\hline County_Smokerate (Ref: <31\%) & & & \\
\hline$>=31 \%$ & $0.823(0.087)$ & $0.669-1.012$ & 0.065 \\
\hline \multicolumn{4}{|l|}{ Overall comorbidity } \\
\hline D’Hoore-CCI & $0.964(0.023)$ & $0.919-1.010$ & 0.122 \\
\hline Number of other chronic conditions & $1.132(0.038)$ & $1.060-1.209$ & $0.000^{*}$ \\
\hline \multicolumn{4}{|l|}{ COPD severity } \\
\hline$\#$ of inhaled SABA canisters & $1.013(0.008)$ & $0.998-1.028$ & 0.098 \\
\hline \# of inhaled IPR canisters & $0.954(0.008)$ & $0.938-0.970$ & $0.000^{*}$ \\
\hline \# of other SABD prescriptions & $0.979(0.009)$ & $0.961-0.998$ & $0.027 *$ \\
\hline \# of OCS prescriptions & $0.998(0.018)$ & $0.963-1.034$ & 0.915 \\
\hline Use of nebulized SABD & $1.201(0.126)$ & $0.984-1.480$ & 0.071 \\
\hline $\begin{array}{r}\text { Presence of Hosp/ER visits for COPD or } \\
\text { COPD-related comorbid disorders }\end{array}$ & $1.241(0.109)$ & $1.044-1.474$ & $0.014^{*}$ \\
\hline \multicolumn{4}{|l|}{$\begin{array}{r}\text { \# of Physician visits for COPD or COPD- } \\
\text { related comorbid disorders }\end{array}$} \\
\hline Presence of asthma & $1.402(0.166)$ & $1.112-1.768$ & $0.004 *$ \\
\hline Use of Home oxygen therapy & $0.809(0.096)$ & $0.640-1.022$ & 0.075 \\
\hline
\end{tabular}


TABLE 47: Propensity score distribution by drug therapy status (ICS vs. no ICS: Phase III Objective 9)

\begin{tabular}{lcc}
\hline Characteristic & ICS & No ICS \\
& $(\mathrm{N}=2,594)$ & $(\mathrm{N}=923)$
\end{tabular}

Propensity score

$$
\text { Mean (SD) } \quad 0.75(0.08) \quad 0.71(0.09)
$$

Propensity score quintile

$$
\begin{array}{ccc}
1 & 0.61(0.08) & 0.60(0.07) \\
2 & 0.70(0.01) & 0.70(0.01) \\
3 & 0.75(0.01) & 0.75(0.01) \\
4 & 0.79(0.01) & 0.78(0.01) \\
5 & 0.84(0.02) & 0.84(0.02) \\
\hline
\end{array}
$$

TABLE 48: Statistical tests of differences in characteristics within each propensity score quintile

\begin{tabular}{lc}
\hline Characteristic & $\begin{array}{c}\text { Test Statistic } \\
\text { (p-value) } \\
*=\text { values }<0.05\end{array}$ \\
\hline Demographic & $0.070^{1}(0.795)$ \\
Age & $0.069^{2}(0.793)$ \\
Female & $0.005^{2}(0.943)$ \\
White & $0.056(0.829)$ \\
State (KY) & $0.032^{2}(0.859)$ \\
Countysmokerate $>=31 \%$ & \\
Overall Comorbidity & $0.000^{1}(0.978)$ \\
D'Hoore-CCI ${ }^{\mathrm{a}}$ & $0.040^{1}(0.845)$ \\
Number of other chronic conditions ${ }^{\mathrm{b}}$ & \\
COPD severity & $0.630^{1}(0.427)$ \\
\# of inhaled SABA ${ }^{\mathrm{c}}$ canisters & $3.300^{1}(0.069)$ \\
\hline \# of inhaled IPR canisters & $180^{\prime}$ \\
\hline
\end{tabular}




\begin{tabular}{|c|c|}
\hline$\#$ of other doseform SABD prescriptions ${ }^{\mathrm{c}}$ & $0.000^{1}(0.995)$ \\
\hline \# of OCS prescriptions ${ }^{\mathrm{d}}$ & $0.000^{1}(0.947)$ \\
\hline Use of nebulized $\mathrm{SABD}^{\mathrm{c}}$ & $0.020^{2}(0.893)$ \\
\hline $\begin{array}{l}\text { Presence of Hosp/ER for COPD or } \\
\text { COPD-related comorbid disorders }\end{array}$ & $0.050^{2}(0.816)$ \\
\hline $\begin{array}{l}\text { \# of Physician visits for COPD or COPD- } \\
\text { related comorbid disorders }{ }^{\mathrm{e}}\end{array}$ & $0.080^{1}(0.780)$ \\
\hline Presence of asthma & $0.030^{2}(0.858)$ \\
\hline Use of Home oxygen therapy & $0.040^{2}(0.850)$ \\
\hline
\end{tabular}

${ }^{1}$ Two-way ANOVA F statistic; ${ }^{2}$ Cochran-Mantel Haenzel test statistic 
TABLE 49: Cox-Proportional hazards regression of the impact of drug therapy (ICS vs. no ICS) on time to death

\begin{tabular}{lllll}
\hline & \multicolumn{3}{c}{ DV: Time to death } \\
Variable & & Hazard ratio (SE) & $95 \%$ CI & $\begin{array}{c}p \\
\text { value }\end{array}$ \\
\hline Drug therapy (Ref: no ICS) & ICS & $0.524(0.130)$ & $0.322-0.852$ & $0.009 *$ \\
Propensity score quintile & & $0.924(0.080)$ & $0.780-1.095$ & 0.361 \\
State (Ref: WV ) & & & & \\
& KY & $0.577(0.138)$ & $0.361-0.923$ & $0.022 *$ \\
\hline
\end{tabular}

$* \mathrm{p}<0.05$

Log likelihood $=-556.48 ; \chi^{2}=13.42 ; \mathrm{p}=0.004 *$

Global test of proportional hazards assumption: $\chi^{2}=1.73 ; p=0.630$

FIGURE 19: Adjusted Kaplan-Meier survival curve of time to death by drug therapy group (ICS vs. no ICS: Phase III - Objective 9)

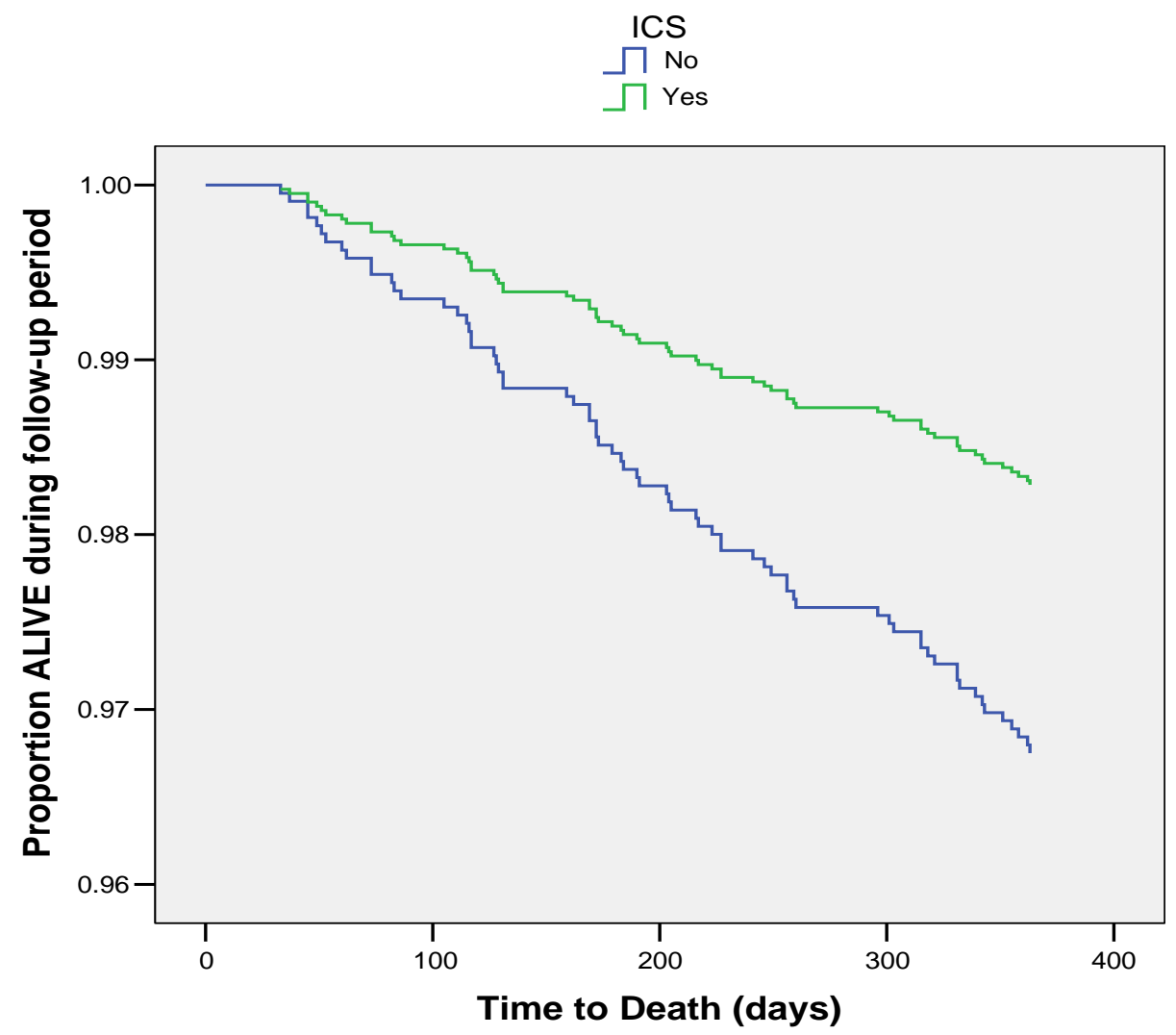


TABLE 50: Cox-Proportional hazards regression of the impact of drug therapy (ICS vs. no ICS) on time to severe exacerbation

\begin{tabular}{lcccc}
\hline \multicolumn{3}{c}{ DV: Time to severe exacerbation } \\
Variable & & Hazard ratio (SE) & $95 \% C I$ & $\begin{array}{c}p \\
\text { value }\end{array}$ \\
\hline Drug therapy (Ref: no ICS) & ICS & $1.186(0.127)$ & $0.961-1.462$ & 0.112 \\
Propensity score quintile & & $1.006(0.032)$ & $0.944-1.071$ & 0.852 \\
State (Ref: $\mathbf{W V})$ & $\mathrm{K}$ & $0.872(0.080)$ & $0.730-1.043$ & 0.135 \\
& KY & & & \\
\hline
\end{tabular}

$* \mathrm{p}<0.05$

Log likelihood $=-3986.97 ; \chi^{2}=5.18 \mathrm{p}=0.159$

Global test of proportional hazards assumption: $\chi^{2}=0.43 ; p=0.934$

FIGURE 20: Adjusted Kaplan-Meier survival curve of time to severe exacerbation by drug therapy group (ICS vs. no ICS: Phase III - Objective 9)

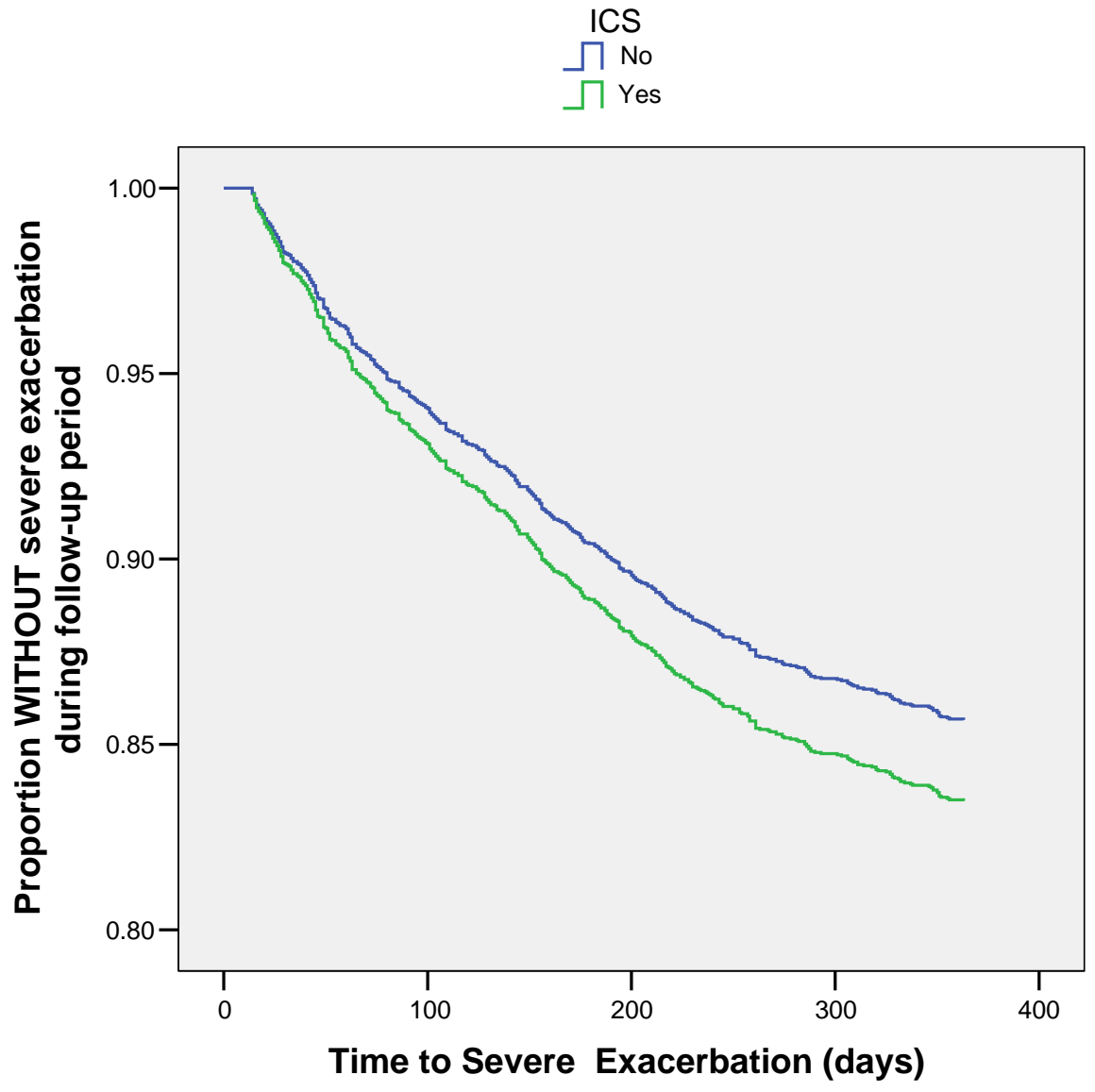




\section{Discussion for Research Objectives 8 and 9}

Research objectives 8 and 9 assessed the impact of ICS on all-cause mortality. The analyses showed ICS to reduce the risk of death by 40 percent using a case-control design, and by 48 percent using a cohort study design in recipients with COPD between 35-64 years of age. Observational studies in the literature have assessed the impact of ICS on mortality in patients with COPD who are generally over 50 years of age. In Ontario, the use of ICS after hospital discharge was associated with a $21 \%$ reduction in mortality over one year of follow-up, whereas in Alberta it was associated with a $25 \%$ reduction in mortality over three years. Soriano et al. analyzed the UK General Practice Research Database (GPRD), and found a 38 percent reduction in mortality over a three-year follow-up period. Another study using HMO claims data in the U.S. found ICS to reduce the risk of death by 41 percent in COPD patients at least 40 years of age.

Currently, a recently published study in September 2006 assessed the impact of ICS on mortality in patients in two age groups, 35-64 years, and $\geq 65$ years. In this study, ICS was found to reduce the risk of death by $25 \%$ in those $>65$ years, and by $53 \%$ in those $35-64$ years using a cohort study design (Macie, Wooldrage, Manfreda, \& Anthonisen, 2006). In the present study, a similarly higher magnitude of risk reduction (48\%) was seen using the cohort study design compared to using the case-control study design.

In contrast to the present study findings and the above study, not all observational studies have found a beneficial effect of ICS on mortality. Two studies used time-dependent methods when analyzing the data to account for immortal time bias (Suissa, 2004; Fan et al., 2003). The results of these studies indicated a trend for reduced mortality that was not significant. However, the use of time-dependent methods has been criticized in situations where protopathic bias might exist (i.e. treatment may be strongly correlated with outcome) (Sin et al., 2003b; Kiri, Vestbo, Pride, \& Soriano, 2004).

The beneficial impact of ICS on mortality that has been seen in the present study and in the majority of observational studies has yet to be validated in clinical trials. Four major clinical trials have found a trend for reduced risk of mortality; however, none found a significant effect (Vestbo et al., 1999; Pauwels et al., 1999; Burge et al., 2000; Lung Health Study Group, 2000). The primary outcome of all these trials was the annual rate of $\mathrm{FEV}_{1}$ decline. None conducted a power analysis to determine if they had sufficient power to detect 
a difference in the mortality outcome. A meta-analysis by Alsaeedi et al. combined data from these trials, and similarly found an overall trend for decreased risk of 0.84 (95\% CI: 0.6 to 1.18) (Alsaeedi et al., 2002). Another scientific review pooled mortality data and found an insignificant reduced risk for mortality of 0.78 (95\%CI: 0.58 to 1.05 ) (Sin et al., 2003c). Preliminary results from the Towards a Revolution in COPD Health (TORCH) trial report that combination ICS/LABA (fluticasone/salmeterol) reduced the risk of all-cause mortality by $17.5 \%$ ( $\mathrm{p}=0.052)$ (Calverley PM, 2006).

Given the significant reduction seen in observational studies, and in the present study, one of the objectives in the present study was also to explain if the reduction in mortality was in fact mediated by the effect of ICS on the risk of severe exacerbations (MacKinnon, 1994). The absence of an effect of ICS on the risk of severe exacerbations in the present study precludes the possibility that ICSs reduce mortality risk by their reduction in severe exacerbations at least in this study. The effect of ICS on mortality in this study can then be explained by examining the causes of death for patients with COPD, and the effect of ICS on these causes. COPD is an independent risk factor for cardiovascular mortality, with cardiovascular diseases accounting for $25 \%$ of all deaths (Anthonisen, Connett, Enright, \& Manfreda, 2002; Zielinski et al., 1997). The link between COPD and cardiovascular events can be attributed to the impact of the primary cause of COPD -- cigarette smoking. Cigarette smoking causes not only lung inflammation but also systemic inflammation (Yasue et al., 2006). Systemic inflammation plays an important role in pathogenesis of atherosclerosis and plaque rupture leading to the increased prevalence of ischemic heart disease (Ross, 1999). There is some evidence that inhaled corticosteroids reduce markers of systemic inflammation (serum C-reactive protein and serum interleukin-6 levels) (Sin, Lacy, York, \& Man, 2004; Sadowska et al., 2005). Thus, it is possible that inhaled corticosteroids reduce systemic inflammation and thereby ischemic events, thus exerting their beneficial effect on mortality. This reasoning seems even more plausible with the publication of two recent studies. The aforementioned study by Macie \& colleagues found ICS to significantly reduce cardiovascular deaths by $38 \%$ in patients over 65 years of age, and no significant reduction for deaths due to COPD and asthma (Macie et al., 2006). A recent study by Huiart et al. has also indicated that therapy with ICS is associated with a decreased risk of myocardial infarction in patients with COPD (Huiart, Ernst, Ranouil, \& Suissa, 2005). 
The relatively lower number of deaths in our study precluded testing whether the reduction in deaths was driven by reduction in cardiovascular deaths. Still, a lower proportion of those using ICS had died from cardiovascular causes compared to those not using ICS (0.009\% vs. $0.014 \%)$. Furthermore, the effect is probably more pronounced in our study population because of the prevalence of cigarette smoking in our study population. As of 2003, Kentucky and West Virginia were ranked \#1 and \#2 in the prevalence of current cigarette smoking in the U.S. (WVBPH, 2005; KYDPH, 2004).

Contrary to the relative risk reduction that gives an indication of statistical significance, the absolute risk reduction obtained from a cohort study design provides information of practical significance. In the present cohort study design, recipients initiated on inhaled corticosteroids had an absolute risk reduction of 1.7 percent, and indicates that 59 people need to be treated with inhaled corticosteroids to avoid one death. It shoud be noted that this effect was not merely restricted to a population of severe to very severe COPD since our study design included recipients with varying severity levels between moderate to very severe COPD. The number needed to treat may be lower in those who are severe. It should also be noted that the incidence of adverse effects was not accounted for in this study, and it is possible that the risk of adverse effects may eliminate the mortality benefit from using inhaled corticosteroids. Thus, this information will be useful to Medicaid policymakers who may want to consider increasing or decreasing the number of recipients being treated with inhaled corticosteroids. 


\section{Chapter 5: SUMmARY AND CONCLUSIONS}

This chapter outlines a summary of the study results, lists study limitations, draws conclusions and implications of the research, and provides recommendations for future research.

\section{Summary of Results for Phase I}

The goal of Phase I was to estimate the costs incurred due to COPD in the West Virginia and Kentucky Medicaid population and identify drivers of cost. The specific impact of concomitant asthma on COPD-related costs was also examined.

Medicaid recipients with COPD incurred 1.5 times significantly higher total healthcare costs than non-COPD recipients with the incremental cost due to COPD per recipient being $\$ 3,952$ and $\$ 4,979$ in $\mathrm{WV}$ and $\mathrm{KY}$, respectively. The greatest difference in costs was due to differences in costs for hospitalizations and ER visits between recipients with and without COPD. Nursing home costs was the only component where recipients with COPD were found to incur lower costs. COPD-related costs accounted for 35 percent of total healthcare costs, indicating that comorbidites have a much greater impact than COPD itself in increasing costs for recipients with COPD recipients.

Recipients with COPD with concomitant asthma were found to incur 50 percent higher costs than recipients with COPD without concomitant asthma. The marginal cost ranged from $\$ 2,200$ in WV Medicaid to $\$ 3,500$ in KY Medicaid. Of note, asthma significantly increased the odds of having a COPD-related hospitalization or ER visit for recipients in both states by 50 percent.

\section{Summary of Results for Phase II}

The goal of Phase II was to assess the cost-effectiveness of maintenance pharmacotherapy used by recipients with COPD. Part 1 compared the risk and frequency of exacerbations, and costs between recipients initiating maintenance therapy with ipratropium (defined as using the combination product of ipratropium/albuterol) or monotherapy with a long-acting beta-agonist in a relatively less severe COPD population. Part 2 compared the risk of a severe exacerbation, frequency of moderate exacerbations, and costs between recipients initiating therapy with a combination of an inhaled corticosteroid with an inhaled 
long-acting beta-agonist, inhaled corticosteroid alone, inhaled long-acting beta-agonist alone, or only short-acting bronchodilators.

\section{Summary of Results for Part 1}

Maintenance therapy with ipratropium was found to be as effective as using an inhaled long-acting beta-agonist in reducing the risk and number of moderate to severe exacerbations during a one year follow-up period for a relatively less severe COPD population. Except for the prescription drug cost component, where recipients initiated on inhaled long-acting betaagonists were found to incur 24 percent (difference of \$140) higher costs, total COPD-related costs incurred during the follow-up period were not significantly different between the two groups. Thus, the pharmacoecomic evaluation did not demonstrate any particular therapy to have a significant advantage over the other.

\section{Summary of Results for Part 2}

Therapy with inhaled corticosteroids either alone or in combination with an inhaled longacting beta-agonist was not found to reduce either the risk of a severe exacerbation or the frequency of moderate exacerbations in comparison to therapy with short-acting bronchodilators. An increase in the number of moderate exacerbations was seen for those using inhaled corticosteroids, but this effect was considered to result from residual confounding rather than due to any detrimental effect of inhaled corticosteroids. The addition of an inhaled corticosteroid to inhaled long-acting beta-agonist monotherapy did not impact the risk of a severe exacerbation or frequency of moderate exacerbations. The comparison of costs incurred during the follow-up reflected the results of the impact of inhaled corticosteroids on exacerbations. Since no difference was seen in exacerbations, no differences were observed in the hospitalization, ER, or outpatient cost components. However, those using inhaled corticosteroids either alone or in combination with an inhaled beta-agonist incurred significantly higher COPD-related prescription drug costs compared to those using short-acting bronchodilators alone, which affected the difference in total COPDrelated costs

\section{Summary of Results for Phase III}

The goal of Phase III was to assess the effect of inhaled corticosteroids on mortality using two study designs: a case-control and a cohort study. Additionally, one of the objectives was 
also to determine if the effect of inhaled corticosteroids on mortality was mediated by the effect of inhaled corticosteroids on severe exacerbations.

Both the case-control and cohort study showed a benefical impact of inhaled corticosteroids on mortality. The magnitude of mortality risk reduction ranged from 40 percent in the case-control study to 48 percent in the cohort study. The risk reduction was significant for the cohort study as the analysis had 95\% power to detect a difference. However, the risk reduction was not statistically significant in the case-control study due to lower power $(45 \%)$. The mortality reduction was not mediated by reducing the risk of severe exacerbations, as inhaled corticosteroids was not shown to have an effect on the risk of severe exacerbation. Contrary to the relative risk reduction that gives an indication of statistical significance, the absolute risk reduction obtained from a cohort study design provides information of practical significance. In the present cohort study design, recipients initiated on inhaled corticosteroids had an absolute risk reduction of 1.7 percent, and indicates that 59 people need to be treated with inhaled corticosteroids to avoid one death.

\section{Study Limitations}

A majority of the limitations in this study can be attributed to the absence of information on important variables in claims databases. Specifically, there was no information on smoking status and use of pulmonary rehabilitation. Both smoking cessation and pulmonary rehabilitation have been shown to impact outcomes of exacerbations and mortality in COPD, and could be potential confounders. Smoking cessation products such as prescription nicotine tablets and nasal sprays could have been obtained from prescription claims. However, the inclusion of this variable does not adequately provide a measure of the smoking status of an individual as those using it are still in the process of quitting. Those not found to be using smoking cessation products can include two groups of COPD patients: those who are still smoking and sustained quitters. Thus, inclusion of this variable would have led to misclassification. Additionally, the claims would not have been able to capture nicotine replacement products that are obtained over-the-counter such as gum and patches.

Studies of drug effects have not shown an interaction effect of smoking and the effect of the drug. However, smoking is an independent predictor of the outcomes of mortality and exacerbations, and could be a confounder. Thus, it is possible that ICS users who were more 
severe than those without ICS may have quit, since most health behavior changes occur when a disease gets more severe. However, in COPD, the percent quitting smoking is low ( $22 \%$ ), and sustained quitters at 11 years were more likely to have higher education among other characateristics (being assigned to a smoking cessation intervention, older age, and smoking fewer cigarettes at baseline) (Murray, Connett, Rand, Pan, \& Anthonisen, 2002). Disease severity as measured by $\mathrm{FEV}_{1}$ or other measure did not predict smoking cessation status. Our study population was restricted to those in a lower socioeconomic status, and therefore likely to have lesser education, and consequently more likely to be smoking. Thus, a strong argument cannot be made that ICS users in Medicaid may have quit smoking solely due to their higher severity.

There was also no information on clinical measures of disease severity, primarily $\mathrm{FEV}_{1}$, and consequently the study methodology could not differentiate recipients who were in stage 3 or stage 4 COPD. This inability might have precluded assessing a beneficial effect of inhaled corticosteroids on exacerbations, since inhaled corticosteroids have been shown to reduce exacerbations in patients with COPD having lower $\mathrm{FEV}_{1}$ levels. Another important aspect influencing outcomes in COPD could not be identified in the prescription claims data, namely the receipt and cost of flu vaccinations. The reason might be that the vaccinations are generally provided in doctor's offices, and are possibly included in the cost of the visit.

In phase II, the pharmacoeconomic evaluation was done comparing therapies that were available during the study time period. However, in 2004 a new long-acting bronchodilator, tiotropium, was introduced that has demonstrated better lung function and lower exacerbation rate compared to ipratropium and placebo. Thus, the cost-effectiveness analysis is limited as this important comparator was not included in the analysis.

Propensity scores were used as an econometric technique to account for selection bias. However, this method can only control for observed factors, and not unobserved factors. Thus, there may still be confounding factors that have not been controlled for leading to residual confounding.

The study population was restricted to a select COPD population enrolled in a Medicaid program from two states in the U.S. that are primarily rural, have the highest smoking rates in the country, and have a high chronic disease burden. Therefore, the study results may not 
be generalizable to findings from other state Medicaid programs that differ in these characteristics.

\section{Conclusions \& Implications of the Research}

The present study showed that recipients with COPD in the Medicaid system had almost 1.5 times significantly higher costs compared to a Medicaid recipient without COPD. This ratio is comparable to the ratio found in other socioeconomic groups that receive healthcare benefits in employer-sponsored insurance programs such as HMOs (Mapel et al., 2000a), and thus the low socioeconomic status of the Medicaid population did not appear to exacerbate the cost burden that may generally be imposed by a patient with COPD. All cost components except for nursing home costs were higher for recipients with COPD compared to those without COPD. The lower nursing home cost can probably be attributed to a policy initiative by Medicaid of reducing costs by shifting to home-based care from nursing home care (Wiener et al., 2002). Kentucky was one of seven states in the U.S. to participate in a home and community based waiver system for older people and younger adults with physical disabilities, and might explain why a significant difference was found in the nursing home cost component as opposed to that seen in the West Virginia sample (Wiener et al., 2002).

A concomitant diagnosis of asthma significantly increased total COPD-related costs for Medicaid recipients with COPD in both states. The incremental cost after adjusting for other respiratory conditions and COPD-related comorbid disorders was still substantial with the difference being almost $50 \%$ higher. The difference in hospitalizations and ER visit costs was largest between those with and without asthma. Thus, policymakers may consider the development of respiratory disease management interventions or other initiatives for this subgroup of recipients with COPD.

Unlike trends in the past decade, prescription drug costs in the present study accounted for a greater proportion of the total costs (almost 25\%) due to the introduction of newer drugs. Our study population was restricted to adults aged between 35 to 64 years excluding those in the Medicare program. Thus, the Medicaid program is still the primary payer for this subset, and should anticipate increasing prescription drug costs for recipients with COPD. An increase is anticipated primarily because of the introduction of a new drug 
tiotropium, and that of another combination product of an inhaled corticosteroid (budesonide) and an inhaled long-acting beta-agonist (formoterol) called Symbicort ${ }^{\circledR}$.

The pharmacoeconomic evaluation of drugs available during the study period found that no drug was significantly better than using short-acting bronchodilators alone in reducing exacerbations of COPD, which is the primary cost driver in COPD. For a recipient with moderate COPD, therapy with ipratropium was found to be equally effective as an inhaled long-acting beta-agonist in reducing exacerbations. Except for higher COPD-related prescription drug costs, total COPD-related costs incurred were found to be similar between therapy groups. Patient preference, satisfactory adherence, and convenience of use (longacting beta-agonists are currently indicated to be used twice a day as opposed to ipratropium which is to be used four times a day) may then be used to facilitate choice of therapy for a relatively less severe COPD population.

COPD is the third or fourth leading cause of death in WV and KY. Therefore, one of the Healthy 2010 objectives for COPD in both states is to reduce the death rate due to COPD, primarily through smoking cessation programs. In the present study, therapy with inhaled corticosteroids was found to reduce mortality rates by almost half. This finding is even more significant considering the younger age group of the study population. Thus, the present study has provided evidence of another treatment modality having a beneficial impact on mortality, and consequently helping to achieve the states' Healthy 2010 objectives.

\section{Recommendations for Future Research}

In the present study, the pharmacoeconomic evaluation did not include an important addition to COPD pharmacotherapy, tiotropium, due to its unavailability during the study period. Future pharmacoeconomic evaluations will be more comprehensive than the present study with the inclusion of this comparator. In the present study, a concomitant diagnosis of asthma was found to significantly increase direct costs for recipients with COPD. However, only direct costs were included as it was relevant to Medicaid. Future studies may want to incorporate an indirect cost component to determine the impact of these concomitant respiratory disorders on work productivity.

Inhaled corticosteroids were shown to reduce mortality, but were not shown to reduce the risk of severe exacerbations. Preliminary hypotheses in the literature suggest that inhaled 
corticosteroids exert their beneficial effect on mortality by reductions in COPD exacerbations which produce hypoxia and instability that may predispose to cardiovascular events, reduction in systemic inflammation, or due to reduced adaptive immune responses (Macie et al., 2006). The current study assessed one of these explanations of whether the reduction in mortality was mediated by the reduction in severe COPD exacerbation. The study did not find any impact. However, the present study design was limited in being able to identify a COPD population specifically with stage 3 and stage 4 , in whom the current literature has found a significant effect on exacerbations. Thus, future studies in severe COPD populations using clinical-level data should be conducted to determine if the mortality reduction is mediated by the impact on exacerbations. To account for smoking status, future claims database research may consider validating the use of smoking cessation aids present in prescription claims data with patient report so that an indicator of smoking status can be created from claims data.

The impact of inhaled corticosteroids on systemic inflammation should also be assessed to determine if mortality is reduced due to the anti-inflammatory properties of inhaled corticosteroids. This mechanism seems more probable because the majority of death reductions are due to cardiovascular deaths. Future research may benefit from conducting a cause-specific mortality analysis. Also, basic clinical research should be conducted to assess the impact of inhaled corticosteroids on markers of systemic inflammation. In addition, a population-based study on the impact of inhaled corticosteroids on cardiovascular events may further support the findings from basic clinical research. 
Bibliography

Alsaeedi, A., Sin, D. D., \& McAlister, F. A. (2002). The effects of inhaled corticosteroids in chronic obstructive pulmonary disease: a systematic review of randomized placebo-controlled trials. $\mathrm{Am} J$ Med, 113, 59-65.

American Lung Association (2004). Trends in chronic bronchitis and emphysema: morbidity and mortality. http://www.lungusa.org/atf/cf/ $\{7 \mathrm{~A} 8 \mathrm{D} 42 \mathrm{C} 2-\mathrm{FCCA}-4604-8 \mathrm{ADE}-$

7F5D5E762256\}/COPD1.PDF [On-line].

American Thoracic Society (1995). Standards for the diagnosis and care of patients with chronic obstructive pulmonary disease. American Thoracic Society. Am.J.Respir.Crit Care Med., 152, S77121.

Anthonisen, N. R., Connett, J. E., Enright, P. L., \& Manfreda, J. (2002). Hospitalizations and mortality in the Lung Health Study. Am J Respir.Crit Care Med, 166, 333-339.

Anthonisen, N. R., Connett, J. E., Kiley, J. P., Altose, M. D., Bailey, W. C., Buist, A. S. et al. (1994). Effects of smoking intervention and the use of an inhaled anticholinergic bronchodilator on the rate of decline of FEV1. The Lung Health Study. JAMA, 272, 1497-1505.

Anthonisen, N. R., Connett, J. E., \& Murray, R. P. (2002). Smoking and lung function of Lung Health Study participants after 11 years. Am J Respir.Crit Care Med, 166, 675-679.

Anthonisen, N. R., Woodlrage, K., \& Manfreda, J. (2005). Use of spirometry and respiratory drugs in Manitobans over 35 years of age with obstructive lung diseases. Can.Respir.J., 12, 69-74.

Appleton, S., Poole, P., Smith, B., Veale, A., \& Bara, A. (2002). Long-acting beta2-agonists for chronic obstructive pulmonary disease patients with poorly reversible airflow limitation.

Cochrane.Database.Syst.Rev., CD001104.

Ayres, J. G., Price, M. J., \& Efthimiou, J. (2003). Cost-effectiveness of fluticasone propionate in the treatment of chronic obstructive pulmonary disease: a double-blind randomized, placebo-controlled trial. Respir.Med., 97, 212-220.

Balzano, G., Stefanelli, F., Iorio, C., De Felice, A., Melillo, E. M., Martucci, M. et al. (1999). Eosinophilic inflammation in stable chronic obstructive pulmonary disease. Relationship with neutrophils and airway function. Am J Respir.Crit Care Med, 160, 1486-1492.

Barnes, P. J. (1998). Efficacy of inhaled corticosteroids in asthma. J Allergy Clin.Immunol., 102, 531538.

Barnes, P. J. (2000a). Inhaled corticosteroids are not beneficial in chronic obstructive pulmonary disease. Am.J.Respir.Crit Care Med., 161, 342-344.

Barnes, P. J. (2000b). Mechanisms in COPD: differences from asthma. Chest, 117, 10S-14S.

Bauer D \& Lavery R (2004). Proc Power in SAS 9.1. http://www2.sas.com/proceedings/sugi29/19529.pdf [On-line]. 
Beauchesne, M. F., Lalande, M., Fillion, A., \& Blais, L. (2005). Inhaled corticosteroids and rate of exacerbations in patients with COPD. Ann.Pharmacother., 39, 973-974.

Behera, D. \& Jindal, S. K. (1991). Respiratory symptoms in Indian women using domestic cooking fuels. Chest, 100, 385-388.

Blais, L., Bourbeau, J., Sheehy, O., \& LeLorier, J. (2004). Inhaled corticosteroids in COPD: determinants of use and trends in patient persistence with treatment. Can.Respir.J., 11, 27-32.

Bloemen, P. G., van den Tweel, M. C., Henricks, P. A., Engels, F., Kester, M. H., van de Loo, P. G. et al. (1997). Increased cAMP levels in stimulated neutrophils inhibit their adhesion to human bronchial epithelial cells. Am J Physiol, 272, L580-L587.

Bourbeau, J., Ernst, P., Cockcoft, D., \& Suissa, S. (2003). Inhaled corticosteroids and hospitalisation due to exacerbation of COPD. Eur.Respir.J, 22, 286-289.

Bourbeau, J., Rouleau, M. Y., \& Boucher, S. (1998). Randomised controlled trial of inhaled corticosteroids in patients with chronic obstructive pulmonary disease. Thorax, 53, 477-482.

Briggs, D. D., Jr. (2004). Chronic obstructive pulmonary disease overview: prevalence, pathogenesis, and treatment. J Manag Care Pharm., 10, S3-10.

Burge, P. S., Calverley, P. M., Jones, P. W., Spencer, S., Anderson, J. A., \& Maslen, T. K. (2000). Randomised, double blind, placebo controlled study of fluticasone propionate in patients with moderate to severe chronic obstructive pulmonary disease: the ISOLDE trial. BMJ, 320, 1297-1303.

Burge, P. S. \& Lewis, S. A. (2003). So inhaled steroids slow the rate of decline of FEV1 in patients with COPD after all? Thorax, 58, 911-913.

Burge, S. \& Wedzicha, J. A. (2003). COPD exacerbations: definitions and classifications.

Eur.Respir.J Suppl, 41, 46s-53s.

Burney, P., Suissa, S., Soriano, J. B., Vollmer, W. M., Viegi, G., Sullivan, S. D. et al. (2003). The pharmacoepidemiology of COPD: recent advances and methodological discussion.

Eur.Respir.J.Suppl, 43, 1s-44s.

Calverley PM (2006). The TOwards A Revolution In COPD Health (TORCH) Study: Fluticasone Propionate/Salmeterol Improves Survival In COPD Over Three Years. Chest, 130, 122S-122Sa.

Calverley, P., Pauwels, R., Vestbo, J., Jones, P., Pride, N., Gulsvik, A. et al. (2003a). Combined salmeterol and fluticasone in the treatment of chronic obstructive pulmonary disease: a randomised controlled trial. Lancet, 361, 449-456.

Calverley, P. M. (2000). Inhaled corticosteroids are beneficial in chronic obstructive pulmonary disease. Am.J.Respir.Crit Care Med., 161, 341-342.

Calverley, P. M., Boonsawat, W., Cseke, Z., Zhong, N., Peterson, S., \& Olsson, H. (2003b). Maintenance therapy with budesonide and formoterol in chronic obstructive pulmonary disease. Eur.Respir.J., 22, 912-919. 
Calverley, P. M., Burge, P. S., Spencer, S., Anderson, J. A., \& Jones, P. W. (2003). Bronchodilator reversibility testing in chronic obstructive pulmonary disease. Thorax, 58, 659-664.

Cazzola, M., Santus, P., di Marco, F., Boveri, B., Castagna, F., Carlucci, P. et al. (2003).

Bronchodilator effect of an inhaled combination therapy with salmeterol + fluticasone and formoterol + budesonide in patients with COPD. Respir.Med, 97, 453-457.

Celli, B. R. (2000). The importance of spirometry in COPD and asthma: effect on approach to management. Chest, 117, 15S-19S.

Celli, B. R. \& MacNee, W. (2004). Standards for the diagnosis and treatment of patients with COPD: a summary of the ATS/ERS position paper. Eur Respir.J, 23, 932-946.

Charlson, M. E., Pompei, P., Ales, K. L., \& MacKenzie, C. R. (1987). A new method of classifying prognostic comorbidity in longitudinal studies: development and validation. J.Chronic.Dis., 40, 373383.

Confalonieri, M., Mainardi, E., Della, P. R., Bernorio, S., Gandola, L., Beghe, B. et al. (1998). Inhaled corticosteroids reduce neutrophilic bronchial inflammation in patients with chronic obstructive pulmonary disease. Thorax, 53, 583-585.

Culpitt, S. V., Maziak, W., Loukidis, S., Nightingale, J. A., Matthews, J. L., \& Barnes, P. J. (1999). Effect of high dose inhaled steroid on cells, cytokines, and proteases in induced sputum in chronic obstructive pulmonary disease. Am J Respir.Crit Care Med, 160, 1635-1639.

D'Agostino, R. B., Jr. (1998). Propensity score methods for bias reduction in the comparison of a treatment to a non-randomized control group. Stat.Med., 17, 2265-2281.

D'Hoore, W., Bouckaert, A., \& Tilquin, C. (1996). Practical considerations on the use of the Charlson comorbidity index with administrative data bases. J.Clin.Epidemiol., 49, 1429-1433.

Dahl, R., Greefhorst, L. A., Nowak, D., Nonikov, V., Byrne, A. M., Thomson, M. H. et al. (2001). Inhaled formoterol dry powder versus ipratropium bromide in chronic obstructive pulmonary disease. Am J Respir.Crit Care Med, 164, 778-784.

Dal Negro, R. W., Pomari, C., Tognella, S., \& Micheletto, C. (2003). Salmeterol \&amp; fluticasone 50 microg $/ 250$ microg bid in combination provides a better long-term control than salmeterol 50 microg bid alone and placebo in COPD patients already treated with theophylline. Pulm.Pharmacol.Ther., 16, 241-246.

de Melo, M. N., Ernst, P., \& Suissa, S. (2004a). Inhaled corticosteroids and the risk of a first exacerbation in COPD patients. Eur.Respir.J, 23, 692-697.

de Melo, M. N., Ernst, P., \& Suissa, S. (2004b). Rates and patterns of chronic obstructive pulmonary disease exacerbations. Can.Respir.J., 11, 559-564.

Decramer, M., Bartsch, P., Pauwels, R., \& Yernault, J. C. (2003). Management of COPD according to guidelines. A national survey among Belgian physicians. Monaldi Arch.Chest Dis., 59, 62-80. 
Decramer, M. \& Selroos, O. (2005). Asthma and COPD: differences and similarities. With special reference to the usefulness of budesonide/formoterol in a single inhaler (Symbicort) in both diseases. Int.J Clin.Pract, 59, 385-398.

Deyo, R. A., Cherkin, D. C., \& Ciol, M. A. (1992). Adapting a clinical comorbidity index for use with ICD-9-CM administrative databases. J.Clin.Epidemiol., 45, 613-619.

Dompeling, E., van Schayck, C. P., van Grunsven, P. M., van Herwaarden, C. L., Akkermans, R., Molema, J. et al. (1993). Slowing the deterioration of asthma and chronic obstructive pulmonary disease observed during bronchodilator therapy by adding inhaled corticosteroids. A 4-year prospective study. Ann.Intern.Med., 118, 770-778.

Donaldson, G. C., Seemungal, T. A., Bhowmik, A., \& Wedzicha, J. A. (2002). Relationship between exacerbation frequency and lung function decline in chronic obstructive pulmonary disease. Thorax, $57,847-852$.

Dougherty, J. A., Didur, B. L., \& Aboussouan, L. S. (2003). Long-acting inhaled beta 2-agonists for stable COPD. Ann.Pharmacother., 37, 1247-1255.

Dowling, R. B., Johnson, M., Cole, P. J., \& Wilson, R. (1998). Effect of salmeterol on Haemophilus influenzae infection of respiratory mucosa in vitro. Eur.Respir.J, 11, 86-90.

Dowling, R. B., Rayner, C. F., Rutman, A., Jackson, A. D., Kanthakumar, K., Dewar, A. et al. (1997). Effect of salmeterol on Pseudomonas aeruginosa infection of respiratory mucosa. Am J Respir.Crit Care Med, 155, 327-336.

Drummond MF, O'Brien BJ, Stoddart GL, \& Torrance GW (1997a). Basic types of economic evaluation. In Drummond M.F., O'Brien B.J., Stoddart G.L., \& Torrance G.W. (Eds.), Methods for the economic evaluation of health care programmes (Second ed., pp. 2-26). New York: Oxford University Press.

Drummond MF, O'Brien BJ, Stoddart GL, \& Torrance GW (1997b). Collection and analysis of data. In Drummond M.F., O'Brien B.J., Stoddart G.L., \& Torrance G.W. (Eds.), Methods for the economic evaluation of health care programmes (Second ed., pp. 232-264). New York: Oxford University Press.

Drummond MF, O'Brien BJ, Stoddart GL, \& Torrance GW (1997c). Cost analysis. In Drummond M.F., O'Brien B.J., Stoddart G.L., \& Torrance G.W. (Eds.), Methods for the economic evaluation of health care programmes (Second ed., pp. 52-95). New York: Oxford University Press.

Duan N (1983). Smearing estimate: A non-parametric retransformation method. Journal of the American Statistical Association, 78, 605-610.

Duan N, Manning WG, Morris CN, \& Newhouse JP (1983). A comparison of alternative models for the demand for medical care. Journal of Business \& Economic Statistics, 1, 115-126.

Dupont, W. D. (1988). Power calculations for matched case-control studies. Biometrics, 44, 1157 1168.

Fabbri, L. M. \& Hurd, S. S. (2003). Global Strategy for the Diagnosis, Management and Prevention of COPD: 2003 update. Eur.Respir.J, 22, 1-2. 
Fan, V. S., Bryson, C. L., Curtis, J. R., Fihn, S. D., Bridevaux, P. O., McDonell, M. B. et al. (2003). Inhaled corticosteroids in chronic obstructive pulmonary disease and risk of death and hospitalization: time-dependent analysis. Am J Respir.Crit Care Med, 168, 1488-1494.

Fan, V. S., Curtis, J. R., Tu, S. P., McDonell, M. B., \& Fihn, S. D. (2002). Using quality of life to predict hospitalization and mortality in patients with obstructive lung diseases. Chest, 122, 429-436.

Friedman, M. (1995). Changing practices in COPD. A new pharmacologic treatment algorithm. Chest, 107, 194S-197S.

Friedman, M. \& Hilleman, D. E. (2001). Economic burden of chronic obstructive pulmonary disease. Impact of new treatment options. Pharmacoeconomics, 19, 245-254.

Friedman, M., Serby, C. W., Menjoge, S. S., Wilson, J. D., Hilleman, D. E., \& Witek, T. J., Jr. (1999). Pharmacoeconomic evaluation of a combination of ipratropium plus albuterol compared with ipratropium alone and albuterol alone in COPD. Chest, 115, 635-641.

Gagnon, Y. M., Levy, A. R., Spencer, M. D., Hurley, J. S., Frost, F. J., Mapel, D. W. et al. (2005). Economic evaluation of treating chronic obstructive pulmonary disease with inhaled corticosteroids and long-acting beta2-agonists in a health maintenance organization. Respir.Med., 99, 1534-1545.

George, D. L. (2004). Chronic obstructive pulmonary disease treatment options. J.Manag.Care Pharm., 10, S11-S16.

GOLD (2004). Global Strategy for the Diagnosis, Management, and Prevention of Chronic Obstructive Pulmonary Disease Updated 2004. NHLBI/WHO workshop report [On-line]. Available: http://www.goldcopd.org/Guidelineitem.asp?11=2\&12=1\&intId $=1388$

Grasso, M. E., Weller, W. E., Shaffer, T. J., Diette, G. B., \& Anderson, G. F. (1998). Capitation, managed care, and chronic obstructive pulmonary disease. Am.J Respir.Crit Care Med, 158, 133-138.

Greene WH. (1994). Accounting for excess zeros and sample selection in Poisson and negative binomial regression models. Working Paper no. EC-94-10.

Ref Type: Unpublished Work

Groenewegen, K. H., Schols, A. M., \& Wouters, E. F. (2003). Mortality and mortality-related factors after hospitalization for acute exacerbation of COPD. Chest, 124, 459-467.

Gross, N. J. (2003). Outcome measurements in COPD: are we schizophrenic? Chest, 123, 1325-1327.

Halpern, M. T., Musin, A., \& Sondhi, S. (2003). Economic analysis of the Confronting COPD survey: methodology. Respir.Med., 97 Suppl C, S15-S22.

Halpern, M. T., Stanford, R. H., \& Borker, R. (2003). The burden of COPD in the U.S.A.: results from the Confronting COPD survey. Respir.Med, 97 Suppl C, S81-S89.

Halvorsen R \& Palmquist R (1980). The interpretation of dummy variables in semilogarithmic equations. American Economic Review, 70, 474-475. 
Hanania, N. A., Darken, P., Horstman, D., Reisner, C., Lee, B., Davis, S. et al. (2003). The efficacy and safety of fluticasone propionate (250 microg)/salmeterol (50 microg) combined in the Diskus inhaler for the treatment of COPD. Chest, 124, 834-843.

Hattotuwa, K. L., Gizycki, M. J., Ansari, T. W., Jeffery, P. K., \& Barnes, N. C. (2002). The effects of inhaled fluticasone on airway inflammation in chronic obstructive pulmonary disease: a double-blind, placebo-controlled biopsy study. Am J Respir.Crit Care Med, 165, 1592-1596.

Highland, K. B., Strange, C., \& Heffner, J. E. (2003). Long-term effects of inhaled corticosteroids on FEV1 in patients with chronic obstructive pulmonary disease. A meta-analysis. Ann Intern.Med, 138, 969-973.

Hilleman, D. E., Dewan, N., Malesker, M., \& Friedman, M. (2000). Pharmacoeconomic evaluation of COPD. Chest, 118, 1278-1285.

Hogan, T. J., Geddes, R., \& Gonzalez, E. R. (2003). An economic assessment of inhaled formoterol dry powder versus ipratropium bromide pressurized metered dose inhaler in the treatment of chronic obstructive pulmonary disease. Clin.Ther., 25, 285-297.

Howard KB, McLaughlin, T., Pathak DS, Okamoto L, \& Bowers B (2001). Cost Analysis: Evaluating Salmeterol Versus Ipratropium to Treat COPD. $P \& T, 26,100-106$.

Hoyert DL, Heron MP, Murphy SL, \& Kung H (2006). Deaths: Final Data for 2003. National vital statistics reports (Rep. No. Vol 54 no 13).

Hyattsville, MD: National Center for Health Statistics.

Huiart, L., Ernst, P., Ranouil, X., \& Suissa, S. (2005). Low-dose inhaled corticosteroids and the risk of acute myocardial infarction in COPD. Eur.Respir.J., 25, 634-639.

Husereau, D., Shukla, V., Boucher, M., Mensinkai, S., \& Dales, R. (2004). Long acting beta2 agonists for stable chronic obstructive pulmonary disease with poor reversibility: a systematic review of randomised controlled trials. BMC.Pulm.Med., 4, 7.

Jackevicius, C., Joyce, D. P., Kesten, S., \& Chapman, K. R. (1997). Prehospitalization inhaled corticosteroid use in patients with COPD or asthma. Chest, 111, 296-302.

Johnson, M. \& Rennard, S. (2001). Alternative mechanisms for long-acting beta(2)-adrenergic agonists in COPD. Chest, 120, 258-270.

Jones, P. W. (1995). Issues concerning health-related quality of life in COPD. Chest, 107, 187S-193S.

Jones, P. W. (2001). Health status measurement in chronic obstructive pulmonary disease. Thorax, $56,880-887$.

Jones, P. W. \& Kaplan, R. M. (2003a). Methodological issues in evaluating measures of health as outcomes for COPD. Eur.Respir.J Suppl, 41, 13s-18s.

Jones, P. W., Willits, L. R., Burge, P. S., \& Calverley, P. M. (2003). Disease severity and the effect of fluticasone propionate on chronic obstructive pulmonary disease exacerbations. Eur.Respir.J., 21, 6873. 
Jones, P. W., Wilson, K., \& Sondhi, S. (2003b). Cost-effectiveness of salmeterol in patients with chronic obstructive pulmonary disease: an economic evaluation. Respir.Med, 97, 20-26.

Jubran, A., Gross, N., Ramsdell, J., Simonian, R., Schuttenhelm, K., Sax, M. et al. (1993). Comparative cost-effectiveness analysis of theophylline and ipratropium bromide in chronic obstructive pulmonary disease. A three-center study. Chest, 103, 678-684.

Kauffmann, F., Drouet, D., Lellouch, J., \& Brille, D. (1979). Twelve years spirometric changes among Paris area workers. Int.J Epidemiol., 8, 201-212.

Keatings, V. M., Jatakanon, A., Worsdell, Y. M., \& Barnes, P. J. (1997). Effects of inhaled and oral glucocorticoids on inflammatory indices in asthma and COPD. Am J Respir.Crit Care Med, 155, 542548.

Kennedy P (1981). Estimation with correctly interpreted dummy variables in semilogarithmic equations. American Economic Review, 71, 801.

Kentucky (2005). Kentucky County Health Profiles. Cabinet for Health and Family Services, Kentucky [On-line].

Kesten S (2001). COPD in a managed care setting. Disease Management and Health Outcomes, 9 , 589-599.

Kesten, S. \& Chapman, K. R. (1993). Physician perceptions and management of COPD. Chest, 104, 254-258.

Kiri, V. A., Vestbo, J., Pride, N. B., \& Soriano, J. B. (2004). Inhaled steroids and mortality in COPD: bias from unaccounted immortal time. Eur.Respir.J, 24, 190-191.

Konzem SL \& Stratton MA (2002). Chronic Obstructive Lung Disease. In DiPiro JT \& Talbert RL (Eds.), Pharmacotherapy: A Pathophysiologic Approach (5th ed., pp. 511-529). New York: McGraw Hill Publishers.

KYDPH (2001). Kentucky Health Behavior Trends 1997-1999. Division of Epidemiology and Planning Kentucky Dept for Public Health [On-line]. Available:

http:/chfs.ky.gov/NR/rdonlyres/A543257C-C7E1-4725-B144-4DFE79E280F7/0/complete.pdf

KYDPH (2002). Kentucky Behavioral Risk Factor Surveillance System 2000 report. Division of Epidemiology and Planning Kentucky Dept for Public Health [On-line]. Available:

http://chfs.ky.gov/NR/rdonlyres/4AF38FF0-5552-447C-8D6D-8790EF76ED8A/0/2000report.pdf

KYDPH (2003). Kentucky Behavioral Risk Factor Surveillance System 2001 report. Division of Epidemiology and Planning Kentucky Dept for Public Health [On-line]. Available:

http://chfs.ky.gov/NR/rdonlyres/F6C0EE67-F23F-4685-9D4A-AF37FA5930B0/0/2001report.pdf

KYDPH (2004). Kentucky Behavioral Risk Factor Surveillance System 2002 report. Division of Epidemiology and Planning Kentucky Dept for Public Health [On-line]. Available:

http://chfs.ky.gov/NR/rdonlyres/38ED0768-1D60-405A-AC2E-4DA764100B41/0/2002report.pdf

Lipson, D. A. (2004). Redefining treatment in COPD: new directions in bronchodilator therapy. Treat.Respir.Med., 3, 89-95. 
Long JS (1997). Regression models for categorical and limited dependent variables. Thousand Oaks, CA: Sage.

Lundback, B., Lindberg, A., Lindstrom, M., Ronmark, E., Jonsson, A. C., Jonsson, E. et al. (2003). Not 15 but $50 \%$ of smokers develop COPD?--Report from the Obstructive Lung Disease in Northern Sweden Studies. Respir.Med., 97, 115-122.

Lung Health Study Group (2000). Effect of inhaled triamcinolone on the decline in pulmonary function in chronic obstructive pulmonary disease. N.Engl.J Med, 343, 1902-1909.

Macie, C., Wooldrage, K., Manfreda, J., \& Anthonisen, N. R. (2006). Inhaled corticosteroids and mortality in COPD. Chest, 130, 640-646.

MacKinnon, D. P. (1994). Analysis of mediating variables in prevention and intervention research. NIDA Res.Monogr, 139, 127-153.

Mahler, D. A., Donohue, J. F., Barbee, R. A., Goldman, M. D., Gross, N. J., Wisniewski, M. E. et al. (1999). Efficacy of salmeterol xinafoate in the treatment of COPD. Chest, 115, 957-965.

Mahler, D. A., Wire, P., Horstman, D., Chang, C. N., Yates, J., Fischer, T. et al. (2002). Effectiveness of fluticasone propionate and salmeterol combination delivered via the Diskus device in the treatment of chronic obstructive pulmonary disease. Am.J.Respir.Crit Care Med., 166, 1084-1091.

Manning WG (1998). The logged dependent variable, heteroskedasticity, and the retransformation problem. Journal of Health Economics, 17, 283-295.

Manning WG \& Mullahy J (2001). Estimating log models: to transform or not to transform? Journal of Health Economics, 20, 461-494.

Mannino, D. M. (2002). COPD: epidemiology, prevalence, morbidity and mortality, and disease heterogeneity. Chest, 121, 121S-126S.

Mannino, D. M. (2003). Chronic obstructive pulmonary disease: definition and epidemiology. Respir.Care, 48, 1185-1191.

Mannino, D. M., Brown, C., \& Giovino, G. A. (1997). Obstructive lung disease deaths in the United States from 1979 through 1993. An analysis using multiple-cause mortality data. Am J Respir.Crit Care Med, 156, 814-818.

Mannino, D. M., Homa, D. M., Akinbami, L. J., Ford, E. S., \& Redd, S. C. (2002). Chronic obstructive pulmonary disease surveillance--United States, 1971-2000. MMWR Surveill Summ., 51, 116.

Mapel, D. W. (2004). Treatment implications on morbidity and mortality in COPD. Chest, 126, 150S$158 \mathrm{~S}$.

Mapel, D. W., Hurley, J. S., Frost, F. J., Petersen, H. V., Picchi, M. A., \& Coultas, D. B. (2000a). Health care utilization in chronic obstructive pulmonary disease. A case-control study in a health maintenance organization. Arch.Intern.Med, 160, 2653-2658. 
Mapel, D. W., Picchi, M. A., Hurley, J. S., Frost, F. J., Petersen, H. V., Mapel, V. M. et al. (2000b). Utilization in COPD: patient characteristics and diagnostic evaluation. Chest, 117, 346S-353S.

Morgan, W. J. (1998). Maternal smoking and infant lung function. Further evidence for an in utero effect. Am J Respir.Crit Care Med, 158, 689-690.

Murray, C. J. \& Lopez, A. D. (1997). Alternative projections of mortality and disability by cause 1990-2020: Global Burden of Disease Study. Lancet, 349, 1498-1504.

Murray, R. P., Connett, J. E., Rand, C. S., Pan, W., \& Anthonisen, N. R. (2002). Persistence of the effect of the Lung Health Study (LHS) smoking intervention over eleven years. Prev Med, 35, 314319.

Murray, R. P., Gerald, L. B., Lindgren, P. G., Connett, J. E., Rand, C. S., \& Anthonisen, N. R. (2000). Characteristics of participants who stop smoking and sustain abstinence for 1 and 5 years in the Lung Health Study. Prev Med, 30, 392-400.

Nannini, L., Lasserson, T. J., \& Poole, P. (2003). Combined corticosteroid and longacting betaagonist in one inhaler for chronic obstructive pulmonary disease. Cochrane.Database.Syst.Rev., CD003794.

NHLBI (2004). Morbidity and Mortality: 2004 Chart Book on Cardiovascular, Lung, and Blood Diseases. http://www.nhlbi.nih.gov/resources/docs/04_chtbk.pdf [On-line].

NHLBI Fact Sheet (2005). National Heart Lung Blood Institute Data Fact Sheet; Chronic Obstructive Pulmonary Disease.

Niederman, M. S., McCombs, J. S., Unger, A. N., Kumar, A., \& Popovian, R. (1999). Treatment cost of acute exacerbations of chronic bronchitis. Clin Ther., 21, 576-591.

Nishimura, K., Izumi, T., Tsukino, M., \& Oga, T. (2002). Dyspnea is a better predictor of 5-year survival than airway obstruction in patients with COPD. Chest, 121, 1434-1440.

O'Donnell, D. E. (2000). Assessment of bronchodilator efficacy in symptomatic COPD: is spirometry useful? Chest, 117, 42S-47S.

Oostenbrink, J. B. \& Rutten-van Molken, M. P. (2004). Resource use and risk factors in high-cost exacerbations of COPD. Respir.Med., 98, 883-891.

Oostenbrink, J. B., Rutten-van Molken, M. P., A1, M. J., Van Noord, J. A., \& Vincken, W. (2004). One-year cost-effectiveness of tiotropium versus ipratropium to treat chronic obstructive pulmonary disease. Eur.Respir.J., 23, 241-249.

Paggiaro, P. L., Dahle, R., Bakran, I., Frith, L., Hollingworth, K., \& Efthimiou, J. (1998). Multicentre randomised placebo-controlled trial of inhaled fluticasone propionate in patients with chronic obstructive pulmonary disease. International COPD Study Group. Lancet, 351, 773-780.

Parsons L.S. (2000). Using SAS software to perform a case-control match on propensity score in an observational study. http://www2.sas.com/proceedings/sugi25/25/po/25p225.pdf [On-line]. 
Pauwels, R. A., Buist, A. S., Calverley, P. M., Jenkins, C. R., \& Hurd, S. S. (2001). Global strategy for the diagnosis, management, and prevention of chronic obstructive pulmonary disease.

NHLBI/WHO Global Initiative for Chronic Obstructive Lung Disease (GOLD) Workshop summary. Am.J.Respir.Crit Care Med., 163, 1256-1276.

Pauwels, R. A., Lofdahl, C. G., Laitinen, L. A., Schouten, J. P., Postma, D. S., Pride, N. B. et al. (1999). Long-term treatment with inhaled budesonide in persons with mild chronic obstructive pulmonary disease who continue smoking. European Respiratory Society Study on Chronic Obstructive Pulmonary Disease. N.Engl.J Med, 340, 1948-1953.

Pauwels, R. A. \& Rabe, K. F. (2004). Burden and clinical features of chronic obstructive pulmonary disease (COPD). Lancet, 364, 613-620.

Pesci, A., Balbi, B., Majori, M., Cacciani, G., Bertacco, S., Alciato, P. et al. (1998). Inflammatory cells and mediators in bronchial lavage of patients with chronic obstructive pulmonary disease.

Eur.Respir.J, 12, 380-386.

Petty, T. L. (1995). The combination of ipratropium and albuterol is more effective than either agent alone. Chest, 107, 183S-186S.

Prescott, E., Godtfredsen, N., Vestbo, J., \& Osler, M. (2003). Social position and mortality from respiratory diseases in males and females. Eur.Respir.J, 21, 821-826.

Prescott, E. \& Vestbo, J. (1999). Socioeconomic status and chronic obstructive pulmonary disease. Thorax, 54, 737-741.

Ramsey, S. D. (2000). Suboptimal medical therapy in COPD: exploring the causes and consequences. Chest, 117, 33S-37S.

Rascati, K. L., Stanford, R. H., \& Borker, R. (2005). A comparison of the risk of hospitalizations due to chronicobstructive pulmonary disease in medicaid patients with various medication regimens, including ipratropium, inhaled corticosteroids, salmeterol, or their combination. Clin Ther., 27, 346354.

Renkema, T. E., Schouten, J. P., Koeter, G. H., \& Postma, D. S. (1996). Effects of long-term treatment with corticosteroids in COPD. Chest, 109, 1156-1162.

Rennard, S. I. (1995). Combination bronchodilator therapy in COPD. Chest, 107, 171S-175S.

Rennard, S. I., Anderson, W., ZuWallack, R., Broughton, J., Bailey, W., Friedman, M. et al. (2001). Use of a long-acting inhaled beta2-adrenergic agonist, salmeterol xinafoate, in patients with chronic obstructive pulmonary disease. Am J Respir.Crit Care Med, 163, 1087-1092.

Rodriguez-Roisin, R. (2000). Toward a consensus definition for COPD exacerbations. Chest, 117, 398S-401S.

Ross, R. (1999). Atherosclerosis--an inflammatory disease. N.Engl.J.Med., 340, 115-126.

Rubin, D. B. (1997). Estimating causal effects from large data sets using propensity scores. Ann.Intern.Med., 127, 757-763. 
Rutschmann, O. T., Janssens, J. P., Vermeulen, B., \& Sarasin, F. P. (2004). Knowledge of guidelines for the management of COPD: a survey of primary care physicians. Respir.Med., 98, 932-937.

Rutten-van Molken, M. P., Van Doorslaer, E. K., Jansen, M. C., Kerstjens, H. A., \& Rutten, F. F. (1995). Costs and effects of inhaled corticosteroids and bronchodilators in asthma and chronic obstructive pulmonary disease. Am.J.Respir.Crit Care Med., 151, 975-982.

Sadowska, A. M., van Overveld, F. J., Gorecka, D., Zdral, A., Filewska, M., Demkow, U. A. et al. (2005). The interrelationship between markers of inflammation and oxidative stress in chronic obstructive pulmonary disease: modulation by inhaled steroids and antioxidant. Respir.Med., 99, 241249.

Samet, J. M., Marbury, M. C., \& Spengler, J. D. (1987). Health effects and sources of indoor air pollution. Part I. Am Rev.Respir.Dis., 136, 1486-1508.

Sclar, D. A., Legg, R. F., Skaer, T. L., Robison, L. M., \& Nemic, N. L. (1994). Ipratropium bromide in the management of chronic obstructive pulmonary disease: effect on health service expenditures. Clin.Ther., 16, 595-601.

Seemungal, T. A., Donaldson, G. C., Paul, E. A., Bestall, J. C., Jeffries, D. J., \& Wedzicha, J. A. (1998). Effect of exacerbation on quality of life in patients with chronic obstructive pulmonary disease. Am.J.Respir.Crit Care Med., 157, 1418-1422.

Sin, D. D., Golmohammadi, K., \& Jacobs, P. (2004). Cost-effectiveness of inhaled corticosteroids for chronic obstructive pulmonary disease according to disease severity. Am J Med, 116, 325-331.

Sin, D. D., Johnson, M., Gan, W. Q., \& Man, S. F. (2004). Combination therapy of inhaled corticosteroids and long-acting beta2-adrenergics in management of patients with chronic obstructive pulmonary disease. Curr.Pharm.Des, 10, 3547-3560.

Sin, D. D., Lacy, P., York, E., \& Man, S. F. (2004). Effects of fluticasone on systemic markers of inflammation in chronic obstructive pulmonary disease. Am.J.Respir.Crit Care Med., 170, 760-765.

Sin, D. D. \& Man, S. F. (2003a). Inhaled corticosteroids and survival in chronic obstructive pulmonary disease: does the dose matter? Eur.Respir.J, 21, 260-266.

Sin, D. D., Man, S. F., \& Tu, J. V. (2003b). Inhaled glucocorticoids in COPD: immortal time bias. Am.J.Respir.Crit Care Med., 168, 126-127.

Sin, D. D., McAlister, F. A., Man, S. F., \& Anthonisen, N. R. (2003c). Contemporary management of chronic obstructive pulmonary disease: scientific review. JAMA, 290, 2301-2312.

Sin, D. D. \& Tu, J. V. (2001). Inhaled corticosteroids and the risk of mortality and readmission in elderly patients with chronic obstructive pulmonary disease. Am J Respir.Crit Care Med, 164, 580584.

Soriano, J. B., Kiri, V. A., Pride, N. B., \& Vestbo, J. (2003). Inhaled corticosteroids with/without long-acting beta-agonists reduce the risk of rehospitalization and death in COPD patients. Am J Respir.Med, 2, 67-74. 
Soriano, J. B., Vestbo, J., Pride, N. B., Kiri, V., Maden, C., \& Maier, W. C. (2002). Survival in COPD patients after regular use of fluticasone propionate and salmeterol in general practice.

Eur.Respir.J, 20, 819-825.

Stein, C. E., Kumaran, K., Fall, C. H., Shaheen, S. O., Osmond, C., \& Barker, D. J. (1997). Relation of fetal growth to adult lung function in south India. Thorax, 52, 895-899.

Strassels, S. A., Smith, D. H., Sullivan, S. D., \& Mahajan, P. S. (2001). The costs of treating COPD in the United States. Chest, 119, 344-352.

Strauss, M. J., Conrad, D., LoGerfo, J. P., Hudson, L. D., \& Bergner, M. (1986). Cost and outcome of care for patients with chronic obstructive lung disease. Analysis by physician specialty. Med.Care, 24, 915-924.

Suissa, S. (2003). Effectiveness of inhaled corticosteroids in chronic obstructive pulmonary disease: immortal time bias in observational studies. Am J Respir.Crit Care Med, 168, 49-53.

Suissa, S. (2004). Inhaled steroids and mortality in COPD: bias from unaccounted immortal time. Eur.Respir.J, 23, 391-395.

Sutherland, E. R., Allmers, H., Ayas, N. T., Venn, A. J., \& Martin, R. J. (2003). Inhaled corticosteroids reduce the progression of airflow limitation in chronic obstructive pulmonary disease: a meta-analysis. Thorax, 58, 937-941.

Szafranski, W., Cukier, A., Ramirez, A., Menga, G., Sansores, R., Nahabedian, S. et al. (2003). Efficacy and safety of budesonide/formoterol in the management of chronic obstructive pulmonary disease. Eur.Respir.J., 21, 74-81.

Tashkin, D. \& Kesten, S. (2003). Long-term treatment benefits with tiotropium in COPD patients with and without short-term bronchodilator responses. Chest, 123, 1441-1449.

Tashkin, D. P. \& Cooper, C. B. (2004). The role of long-acting bronchodilators in the management of stable COPD. Chest, 125, 249-259.

Tennant, R. C., Erin, E. M., Barnes, P. J., \& Hansel, T. T. (2003). Long-acting beta 2-adrenoceptor agonists or tiotropium bromide for patients with COPD: is combination therapy justified?

Curr.Opin.Pharmacol., 3, 270-276.

Todisco, T., de Benedictis, F. M., Iannacci, L., Baglioni, S., Eslami, A., Todisco, E. et al. (1993). Mild prematurity and respiratory functions. Eur.J Pediatr., 152, 55-58.

Trakada, G. \& Spiropoulos, K. (2000). Chronic obstructive pulmonary disease management among primary healthcare physicians. Monaldi Arch.Chest Dis., 55, 201-204.

Van Andel, A. E., Reisner, C., Menjoge, S. S., \& Witek, T. J. (1999). Analysis of inhaled corticosteroid and oral theophylline use among patients with stable COPD from 1987 to 1995. Chest, $115,703-707$.

van Grunsven, P. M., van Schayck, C. P., Derenne, J. P., Kerstjens, H. A., Renkema, T. E., Postma, D. S. et al. (1999). Long term effects of inhaled corticosteroids in chronic obstructive pulmonary disease: a meta-analysis. Thorax, 54, 7-14. 
van, d., V, Monninkhof, E., van der, P. J., Zielhuis, G., \& van Herwaarden, C. (2002). Effect of discontinuation of inhaled corticosteroids in patients with chronic obstructive pulmonary disease: the COPE study. Am.J.Respir.Crit Care Med., 166, 1358-1363.

Vestbo, J. \& Lange, P. (2002). Can GOLD Stage 0 provide information of prognostic value in chronic obstructive pulmonary disease? Am J Respir.Crit Care Med, 166, 329-332.

Vestbo, J., Sorensen, T., Lange, P., Brix, A., Torre, P., \& Viskum, K. (1999). Long-term effect of inhaled budesonide in mild and moderate chronic obstructive pulmonary disease: a randomised controlled trial. Lancet, 353, 1819-1823.

Wadbo, M., Lofdahl, C. G., Larsson, K., Skoogh, B. E., Tornling, G., Arwestrom, E. et al. (2002). Effects of formoterol and ipratropium bromide in COPD: a 3-month placebo-controlled study. Eur.Respir.J., 20, 1138-1146.

Ward, M. M., Javitz, H. S., Smith, W. M., \& Bakst, A. (2000). Direct medical cost of chronic obstructive pulmonary disease in the U.S.A. Respir.Med, 94, 1123-1129.

Wedzicha, J. A. \& Donaldson, G. C. (2003). Exacerbations of chronic obstructive pulmonary disease. Respir.Care, 48, 1204-1213.

Weir, D. C., Bale, G. A., Bright, P., \& Sherwood, B. P. (1999). A double-blind placebo-controlled study of the effect of inhaled beclomethasone dipropionate for 2 years in patients with nonasthmatic chronic obstructive pulmonary disease. Clin.Exp.Allergy, 29 Suppl 2, 125-128.

West Virginia (2005). West Virginia and West Virginians. West Virginia HealthCare Authority [Online].

Wiener, J. M., Tilly, J., \& Alecxih, L. M. (2002). Home and community-based services in seven states. Health Care Financ.Rev., 23, 89-114.

Wilson, L., Devine, E. B., \& So, K. (2000). Direct medical costs of chronic obstructive pulmonary disease: chronic bronchitis and emphysema. Respir.Med, 94, 204-213.

WVBPH (2005). 2003 West Virginia Behavioral Risk Factor Survey Report. West Virginia Dept of Health and Human Services Bureau for Public Health [On-line]. Available:

http://www.wvdhhr.org/bph/oehp/BRFSS 2003/

Yasue, H., Hirai, N., Mizuno, Y., Harada, E., Itoh, T., Yoshimura, M. et al. (2006). Low-grade inflammation, thrombogenicity, and atherogenic lipid profile in cigarette smokers. Circ.J., 70, 8-13.

Yildiz, F., Kaur, A. C., Ilgazli, A., Celikoglu, M., Kacar, O. S., Paksoy, N. et al. (2000). Inhaled corticosteroids may reduce neutrophilic inflammation in patients with stable chronic obstructive pulmonary disease. Respiration, 67, 71-76.

Zielinski, J., MacNee, W., Wedzicha, J., Ambrosino, N., Braghiroli, A., Dolensky, J. et al. (1997). Causes of death in patients with COPD and chronic respiratory failure. Monaldi Arch.Chest Dis., 52, 43-47. 
Appendix A: Regression Models for Analysis of Cost Data for Phase I 
TABLE A1: [WV Medicaid] Two-part model of the impact of COPD on hospitalization costs

\begin{tabular}{|c|c|c|c|c|}
\hline \multirow[b]{3}{*}{ Variable } & \multicolumn{4}{|c|}{ DV: Hospitalization costs } \\
\hline & \multicolumn{2}{|l|}{ Part $1^{\text {a }}$} & \multicolumn{2}{|l|}{ Part $2^{\mathrm{b}}$} \\
\hline & $\begin{array}{l}\text { Odds Ratio } \\
\text { (SE) }\end{array}$ & $\begin{array}{c}p \\
\text { value }\end{array}$ & $\begin{array}{l}\beta \text { coefficient } \\
(S E)\end{array}$ & $\begin{array}{c}p \\
\text { value }\end{array}$ \\
\hline \multicolumn{5}{|l|}{ COPD (Ref: No) } \\
\hline Yes & $3.635(0.190)$ & $0.000 *$ & $0.101(0.031)$ & $0.001 *$ \\
\hline \multicolumn{5}{|l|}{ Demographic } \\
\hline \multicolumn{5}{|l|}{ Race (Ref: non-White) } \\
\hline White & $0.899(0.109)$ & 0.379 & $0.028(0.064)$ & 0.663 \\
\hline \multicolumn{4}{|l|}{ County_Smokerate(Re } & \\
\hline$>=31 \%$ & $0.705(0.043)$ & $0.000 *$ & $-0.009(0.033)$ & 0.783 \\
\hline
\end{tabular}

\section{Overall comorbidity}

$\begin{array}{rllll}\text { D'Hoore-CCI } & 1.446(0.019) & 0.000^{*} & 0.095(0.005) & 0.000^{*} \\ \begin{array}{c}\text { Number of other } \\ \text { chronic conditions }\end{array} & 1.365(0.027) & 0.000 * & 0.078(0.100) & 0.000^{*}\end{array}$

$* \mathrm{p}<0.05$

${ }^{a}$ Part 1: Logistic regression: $-2 \log$ likelihood $=-4814.97, \chi^{2}=2841.38, \mathrm{p}=0.000$; Pseudo $\mathrm{R}^{2}=22.78 \%$

${ }^{\mathrm{b}}$ Part 2: GLM family (gamma) $\operatorname{link}(\log )$ : AIC $=20.04$ Deviance=1482.27; $(1 / \mathrm{df})$ Deviance $=0.48$ 
TABLE A2: [WV Medicaid] Two-part model of the impact of COPD on ER costs

\begin{tabular}{|c|c|c|c|c|}
\hline \multirow[b]{3}{*}{ Variable } & \multicolumn{4}{|l|}{ DV: ER costs } \\
\hline & \multicolumn{2}{|l|}{ Part $1^{\mathrm{a}}$} & \multicolumn{2}{|l|}{ Part $2^{\mathrm{b}}$} \\
\hline & $\begin{array}{l}\text { Odds Ratio } \\
(S E)\end{array}$ & $\begin{array}{c}p \\
\text { value }\end{array}$ & $\begin{array}{l}\beta \text { coefficient } \\
(S E)\end{array}$ & $\begin{array}{c}p \\
\text { value }\end{array}$ \\
\hline \multicolumn{5}{|l|}{ COPD (Ref: No) } \\
\hline Yes & $1.853(0.080)$ & $0.000^{*}$ & $0.295(0.028)$ & $0.000 *$ \\
\hline \multicolumn{5}{|l|}{ Demographic } \\
\hline \multicolumn{5}{|l|}{ Race (Ref: non-White) } \\
\hline White & $0.800(0.081)$ & $0.028^{*}$ & $-0.159(0.062)$ & $0.010^{*}$ \\
\hline \multicolumn{5}{|l|}{ County_Smokerate(Ref: } \\
\hline$>=31 \%$ & $0.904(0.045)$ & $0.044 *$ & $0.133(0.032)$ & $0.000 *$ \\
\hline
\end{tabular}

\section{Overall comorbidity}

\begin{tabular}{rllll} 
D'Hoore-CCI & $1.190(0.014)$ & $0.000^{*}$ & $0.082(0.006)$ & $0.000^{*}$ \\
$\begin{array}{r}\text { Number of other } \\
\text { chronic conditions }\end{array}$ & $1.268(0.022)$ & $0.000^{*}$ & $0.204(0.010)$ & $0.000^{*}$ \\
\hline
\end{tabular}

$* \mathrm{p}<0.05$

${ }^{a}$ Part 1: Logistic regression: $-2 \log$ likelihood $=-6312.68, \chi^{2}=1107.01, p=0.000$; Pseudo $R^{2}=8.06 \%$

${ }^{\mathrm{b}}$ Part 2: GLM family (gamma) link $(\log )$ : AIC $=15.02$ Deviance=5000.48; $(1 / \mathrm{df})$ Deviance $=1.05$ 
TABLE A3: [WV Medicaid] Generalized linear model (GLM) of the impact of COPD on Outpatient costs

\begin{tabular}{|c|c|c|}
\hline \multirow[b]{2}{*}{ Variable } & \multicolumn{2}{|c|}{ DV: Outpatient costs } \\
\hline & $\begin{array}{l}\beta \text { coefficient } \\
(S E)\end{array}$ & $\begin{array}{c}p \\
\text { value }\end{array}$ \\
\hline \multicolumn{3}{|l|}{ COPD (Ref: No) } \\
\hline Yes & $0.494(0.021)$ & $0.000 *$ \\
\hline \multicolumn{3}{|l|}{ Demographic } \\
\hline \multicolumn{3}{|l|}{ Race (Ref: non-White) } \\
\hline White & $0.144(0.050)$ & $0.004 *$ \\
\hline \multicolumn{3}{|l|}{ County Smokerate(Ref: } \\
\hline$>=31 \%$ & $-0.081(0.001)$ & $0.001 *$ \\
\hline
\end{tabular}

Overall comorbidity

$\begin{array}{rcc}\text { D'Hoore-CCI } & 0.157(0.005) & 0.000^{*} \\ \begin{array}{r}\text { Number of other chronic } \\ \text { conditions }^{\mathrm{a}}\end{array} & 0.243(0.008) & 0.000^{*} \\ & & \end{array}$

$* \mathrm{p}<0.05$

GLM [family (gamma) $\operatorname{link}(\log )]$ : AIC $=17.05 ;$ Deviance=12761.28; $(1 / \mathrm{df})$ Deviance $=1.32$ 
TABLE A4: [WV Medicaid] Two-part model of the impact of COPD on Physician visit costs

\begin{tabular}{|c|c|c|c|c|}
\hline \multirow[b]{3}{*}{ Variable } & \multicolumn{4}{|c|}{ DV: Physician visit costs } \\
\hline & \multicolumn{2}{|l|}{ Part $1^{\mathrm{a}}$} & \multicolumn{2}{|l|}{ Part $2^{\mathrm{b}}$} \\
\hline & $\begin{array}{l}\text { Odds Ratio } \\
(S E)\end{array}$ & $\begin{array}{c}p \\
\text { value }\end{array}$ & $\begin{array}{l}\beta \text { coefficient } \\
(S E)\end{array}$ & $\begin{array}{c}p \\
\text { value }\end{array}$ \\
\hline \multicolumn{5}{|l|}{ COPD (Ref: No) } \\
\hline Yes & $1.684(0.105)$ & $0.000 *$ & $0.173(0.031)$ & $0.000^{*}$ \\
\hline \multicolumn{5}{|l|}{ Demographic } \\
\hline \multicolumn{5}{|l|}{ Race (Ref: non-White) } \\
\hline White & $0.888(0.127)$ & 0.405 & $0.130(0.073)$ & 0.073 \\
\hline \multicolumn{5}{|l|}{ County_Smokerate(Re } \\
\hline$>=31 \%$ & $0.806(0.055)$ & $0.002 *$ & $0.164(0.036)$ & $0.000 *$ \\
\hline
\end{tabular}

Overall comorbidity

$\begin{array}{rrrrr}\text { D'Hoore-CCI } & 1.231(0.026) & 0.000 * & 0.122(0.007) & 0.000^{*} \\ \begin{array}{r}\text { Number of other } \\ \text { chronic conditions }\end{array} & 1.544(0.042) & 0.000 * & 0.269(0.012) & 0.000^{*}\end{array}$

$* \mathrm{p}<0.05$

${ }^{a}$ Part 1: Logistic regression: $-2 \log$ likelihood $=-3719.34, \chi^{2}=787.30, p=0.000$; Pseudo $\mathrm{R}^{2}=9.57 \%$

${ }^{\mathrm{b}}$ Part 2: Semi- $\log$ OLS: $\mathrm{R}^{2}=14.05 \%$; $\mathrm{p}=0.000$; Test for heteroskedasticity $\left(\chi^{2}=0.16, \mathrm{p}=0.692\right)$ 
TABLE A5: [WV Medicaid] Two-part model of the impact of COPD on Prescription drug costs

\begin{tabular}{|c|c|c|c|c|}
\hline \multirow[b]{3}{*}{ Variable } & \multicolumn{4}{|c|}{ DV: Physician visit costs } \\
\hline & \multicolumn{2}{|l|}{ Part $1^{\text {a }}$} & \multicolumn{2}{|l|}{ Part $2^{\mathrm{b}}$} \\
\hline & $\begin{array}{l}\text { Odds Ratio } \\
(S E)\end{array}$ & $\begin{array}{c}p \\
\text { value }\end{array}$ & $\begin{array}{l}\beta \text { coefficient } \\
(S E)\end{array}$ & $\begin{array}{c}p \\
\text { value }\end{array}$ \\
\hline \multicolumn{5}{|l|}{ COPD (Ref: No) } \\
\hline Yes & $1.095(0.083)$ & 0.232 & $0.304(0.019)$ & $0.000^{*}$ \\
\hline \multicolumn{5}{|l|}{ Demographic } \\
\hline \multicolumn{5}{|l|}{ Race (Ref: non-White) } \\
\hline White & $1.358(0.211)$ & $0.048 *$ & $0.164(0.046)$ & $0.000 *$ \\
\hline \multicolumn{4}{|l|}{ County_Smokerate(Re } & \\
\hline$>=31 \%$ & $1.358(0.128)$ & $0.001 *$ & $-0.092(0.022)$ & $0.000 *$ \\
\hline
\end{tabular}

Overall comorbidity

$\begin{array}{rrrrr}\text { D'Hoore-CCI } & 1.043(0.021) & 0.040^{*} & 0.078(0.005) & 0.000^{*} \\ \begin{array}{r}\text { Number of other } \\ \text { chronic conditions }\end{array} & 1.375(0.044) & 0.000 * & 0.154(0.008) & 0.000^{*}\end{array}$

$* \mathrm{p}<0.05$

${ }^{a}$ Part 1: Logistic regression: $-2 \log$ likelihood $=-2744.33, \chi^{2}=173.40, p=0.000$; Pseudo $R^{2}=3.06 \%$

${ }^{\mathrm{b}}$ Part 2: GLM family (gamma) $\operatorname{link}(\log ): \mathrm{AIC}=18.25$ Deviance=7716.10; $(1 / \mathrm{df})$ Deviance $=0.84$ 
TABLE A6: [WV Medicaid] Two-part model of the impact of COPD on Nursing home costs

\begin{tabular}{|c|c|c|c|c|}
\hline \multirow[b]{3}{*}{ Variable } & \multicolumn{4}{|c|}{ DV: Nursing home costs } \\
\hline & \multicolumn{2}{|l|}{ Part $1^{\mathrm{a}}$} & \multicolumn{2}{|l|}{ Part $2^{\mathrm{b}}$} \\
\hline & $\begin{array}{l}\text { Odds Ratio } \\
(S E)\end{array}$ & $\begin{array}{c}p \\
\text { value }\end{array}$ & $\begin{array}{l}\beta \text { coefficient } \\
(S E)\end{array}$ & $\begin{array}{c}p \\
\text { value }\end{array}$ \\
\hline \multicolumn{5}{|l|}{ COPD (Ref: No) } \\
\hline Yes & $0.722(0.078)$ & $0.003 *$ & $-0.126(0.075)$ & 0.092 \\
\hline \multicolumn{5}{|l|}{ Demographic } \\
\hline \multicolumn{5}{|l|}{ Race (Ref: non-White) } \\
\hline White & $1.451(0.394)$ & 0.171 & $-0.158(0.172)$ & 0.357 \\
\hline \multicolumn{5}{|l|}{ County_Smokerate(Re } \\
\hline$>=31 \%$ & $0.709(0.095)$ & $0.011 *$ & $0.192(0.087)$ & $0.027 *$ \\
\hline
\end{tabular}

\section{Overall comorbidity}

$\begin{array}{rllll}\text { D'Hoore-CCI } & 1.271(0.023) & 0.000 * & -0.062(0.012) & 0.000^{*} \\ \begin{array}{r}\text { Number of other } \\ \text { chronic conditions }\end{array} & 0.863(0.035) & 0.000 * & -0.097(0.026) & 0.000^{*}\end{array}$

$* \mathrm{p}<0.05$

${ }^{a}$ Part 1: Logistic regression: $-2 \log$ likelihood $=-1599.97, \chi^{2}=157.94, p=0.000$; Pseudo $\mathrm{R}^{2}=4.70 \%$

${ }^{\mathrm{b}}$ Part 2: GLM family (gamma) link $(\log ): \mathrm{AIC}=22.86$ Deviance=297.86; $(1 / \mathrm{df})$ Deviance $=0.78$ 
TABLE A7: [WV Medicaid] Generalized linear model (GLM) of the impact of COPD on total healthcare costs

\begin{tabular}{|c|c|c|}
\hline \multirow[b]{2}{*}{ Variable } & \multicolumn{2}{|c|}{ DV: Total healthcare costs } \\
\hline & $\begin{array}{l}\beta \text { coefficient } \\
(S E)\end{array}$ & $\begin{array}{c}p \\
\text { value }\end{array}$ \\
\hline \multicolumn{3}{|l|}{ COPD (Ref: No) } \\
\hline Yes & $0.551(0.018)$ & $0.000^{*}$ \\
\hline \multicolumn{3}{|l|}{ Demographic } \\
\hline \multicolumn{3}{|l|}{ Race (Ref: non-White) } \\
\hline White & $0.107(0.042)$ & $0.012 *$ \\
\hline \multicolumn{3}{|l|}{ County_Smokerate(Ref: } \\
\hline$>=31 \%$ & $-0.123(0.209)$ & $0.000 *$ \\
\hline
\end{tabular}

\section{Overall comorbidity}

\begin{tabular}{rcc} 
D'Hoore-CCI & $0.190(0.004)$ & $0.000 *$ \\
$\begin{array}{r}\text { Number of other chronic } \\
\text { conditions }^{\mathrm{a}}\end{array}$ & $0.229(0.007)$ & $0.000 *$ \\
\hline *p<0.05 \\
GLM [family (gamma) link(log)]: AIC $=19.74 ;$ Deviance $=8630.85 ;(1 / \mathrm{df})$ Deviance $=0.91$.
\end{tabular}


TABLE A8: [KY Medicaid] Two-part model of the impact of COPD on hospitalization costs

\begin{tabular}{|c|c|c|c|c|}
\hline \multirow[b]{3}{*}{ Variable } & \multicolumn{4}{|c|}{ DV: Nursing home costs } \\
\hline & \multicolumn{2}{|l|}{ Part $1^{\mathrm{a}}$} & \multicolumn{2}{|l|}{ Part $2^{\mathrm{b}}$} \\
\hline & $\begin{array}{l}\text { Odds Ratio } \\
(S E)\end{array}$ & $\begin{array}{c}p \\
\text { value }\end{array}$ & $\begin{array}{l}\beta \text { coefficient } \\
(S E)\end{array}$ & $\begin{array}{c}p \\
\text { value }\end{array}$ \\
\hline \multicolumn{5}{|l|}{ COPD (Ref: No) } \\
\hline Yes & $4.328(0.215)$ & $0.000 *$ & $0.236(0.039)$ & $0.000^{*}$ \\
\hline \multicolumn{5}{|l|}{ Demographic } \\
\hline \multicolumn{5}{|l|}{ Race (Ref: non-White) } \\
\hline White & $0.918(0.103)$ & 0.448 & $-0.149(0.079)$ & 0.061 \\
\hline \multicolumn{4}{|l|}{ County_Smokerate(Re } & \\
\hline$>=31 \%$ & $1.163(0.086)$ & $0.041 *$ & $-0.048(0.052)$ & 0.358 \\
\hline
\end{tabular}

\section{Overall comorbidity}

$\begin{array}{rllll}\text { D'Hoore-CCI } & 1.426(0.018) & 0.000 * & 0.139(0.006) & 0.000^{*} \\ \begin{array}{c}\text { Number of other } \\ \text { chronic conditions }\end{array} & 1.401(0.025) & 0.000 * & 0.031(0.012) & 0.011^{*}\end{array}$

$* \mathrm{p}<0.05$

${ }^{a}$ Part 1: Logistic regression: $-2 \log$ likelihood $=-5722.11, \chi^{2}=3563.75, \mathrm{p}=0.000$; Pseudo $\mathrm{R}^{2}=23.75 \%$

${ }^{\mathrm{b}}$ Part 2: Semi-log OLS: $\mathrm{R}^{2}=14.39 \% ; \mathrm{p}=0.000$; Test for heteroskedasticity $\left(\chi^{2}=18.61, \mathrm{p}=0.351\right)$ 
TABLE A9: [KY Medicaid] Two-part model of the impact of COPD on ER costs

\begin{tabular}{|c|c|c|c|c|}
\hline \multirow[b]{3}{*}{ Variable } & \multicolumn{4}{|l|}{ DV: ER costs } \\
\hline & \multicolumn{2}{|l|}{ Part $1^{\mathrm{a}}$} & \multicolumn{2}{|l|}{ Part $2^{\mathrm{b}}$} \\
\hline & $\begin{array}{l}\text { Odds Ratio } \\
(S E)\end{array}$ & $\begin{array}{c}p \\
\text { value }\end{array}$ & $\begin{array}{l}\beta \text { coefficient } \\
(S E)\end{array}$ & $\begin{array}{c}p \\
\text { value }\end{array}$ \\
\hline \multicolumn{5}{|l|}{ COPD (Ref: No) } \\
\hline Yes & $2.446(0.097)$ & $0.000 *$ & $0.413(0.029)$ & $0.000^{*}$ \\
\hline \multicolumn{5}{|l|}{ Demographic } \\
\hline \multicolumn{5}{|l|}{ Race (Ref: non-White) } \\
\hline White & $0.817(0.072)$ & $0.022 *$ & $-0.142(0.061)$ & $0.020 *$ \\
\hline \multicolumn{4}{|l|}{ County_Smokerate(Re } & \\
\hline$>=31 \%$ & $0.845(0.050)$ & $0.004 *$ & $0.111(0.040)$ & 0.777 \\
\hline
\end{tabular}

\section{Overall comorbidity}

$\begin{array}{rcccc}\text { D'Hoore-CCI } & 1.143(0.012) & 0.000^{*} & 0.083(0.006) & 0.000^{*} \\ \begin{array}{c}\text { Number of other } \\ \text { chronic conditions }\end{array} & 1.314(0.020) & 0.000^{*} & 0.160(0.010) & 0.000^{*}\end{array}$

$* \mathrm{p}<0.05$

${ }^{a}$ Part 1: Logistic regression: $-2 \log$ likelihood $=-7700.19, \chi^{2}=1655.41, p=0.000$; Pseudo $R^{2}=9.71 \%$

${ }^{\mathrm{b}}$ Part 2: GLM family (gamma) $\operatorname{link}(\log ): \mathrm{AIC}=15.66$ Deviance=4913.38; $(1 / \mathrm{df})$ Deviance $=0.90$ 
TABLE A10: [KY Medicaid] Generalized linear model (GLM) of the impact of COPD on Outpatient costs

\begin{tabular}{|c|c|c|}
\hline \multirow[b]{2}{*}{ Variable } & \multicolumn{2}{|c|}{ DV: Outpatient costs } \\
\hline & $\begin{array}{l}\beta \text { coefficient } \\
(S E)\end{array}$ & $\begin{array}{c}p \\
\text { value }\end{array}$ \\
\hline \multicolumn{3}{|l|}{ COPD (Ref: No) } \\
\hline Yes & $0.425(0.024)$ & $0.000^{*}$ \\
\hline \multicolumn{3}{|l|}{ Demographic } \\
\hline \multicolumn{3}{|l|}{ Race (Ref: non-White) } \\
\hline White & $0.036(0.055)$ & 0.518 \\
\hline \multicolumn{3}{|l|}{ County_Smokerate(Ref: } \\
\hline$>=31 \%$ & $-0.168(0.036)$ & $0.000 *$ \\
\hline
\end{tabular}

Overall comorbidity

$\begin{array}{rcc}\text { D'Hoore-CCI } & 0.218(0.007) & 0.000^{*} \\ \begin{array}{r}\text { Number of other chronic } \\ \text { conditions }^{\mathrm{a}}\end{array} & 0.132(0.009) & 0.000^{*} \\ & & \end{array}$

$* \mathrm{p}<0.05$

GLM [family (gamma) $\operatorname{link}(\log )]$ : AIC $=18.27 ;$ Deviance $=18340.62 ;(1 / \mathrm{df})$ Deviance $=1.55$ 
TABLE A11: [KY Medicaid] Two-part model of the impact of COPD on Physician costs

\begin{tabular}{|c|c|c|c|c|}
\hline \multirow[b]{3}{*}{ Variable } & \multicolumn{4}{|c|}{ DV: Physician costs } \\
\hline & \multicolumn{2}{|l|}{ Part $1^{\mathrm{a}}$} & \multicolumn{2}{|l|}{ Part $2^{\mathrm{b}}$} \\
\hline & $\begin{array}{l}\text { Odds Ratio } \\
(S E)\end{array}$ & $\begin{array}{c}p \\
\text { value }\end{array}$ & $\begin{array}{l}\beta \text { coefficient } \\
(S E)\end{array}$ & $\begin{array}{c}p \\
\text { value }\end{array}$ \\
\hline \multicolumn{5}{|l|}{ COPD (Ref: No) } \\
\hline Yes & $2.940(0.193)$ & $0.000 *$ & $0.393(0.016)$ & $0.000^{*}$ \\
\hline \multicolumn{5}{|l|}{ Demographic } \\
\hline \multicolumn{5}{|l|}{ Race (Ref: non-White) } \\
\hline White & $1.165(0.136)$ & 0.191 & $0.086(0.037)$ & $0.022 *$ \\
\hline \multicolumn{5}{|l|}{ County_Smokerate(Re } \\
\hline$>=31 \%$ & $1.236(0.100)$ & $0.009 *$ & $0.035(0.024)$ & 0.154 \\
\hline
\end{tabular}

\section{Overall comorbidity}

$\begin{array}{rllll}\text { D'Hoore-CCI } & 1.042(0.021) & 0.039 * & 0.129(0.004) & 0.000^{*} \\ \begin{array}{c}\text { Number of other } \\ \text { chronic conditions }\end{array} & 3.767(0.146) & 0.000 * & 0.213(0.006) & 0.000^{*}\end{array}$

$* \mathrm{p}<0.05$

${ }^{a}$ Part 1: Logistic regression: $-2 \log$ likelihood $=-3794.30, \chi^{2}=2888.28, \mathrm{p}=0.000$; Pseudo $\mathrm{R}^{2}=27.57 \%$

${ }^{\mathrm{b}}$ Part 2: GLM family (gamma) link $(\log ):$ AIC $=14.92$ Deviance=6934.20; $(1 / \mathrm{df})$ Deviance $=0.69$ 
TABLE A12: [KY Medicaid] Two-part model of the impact of COPD on Prescription drug costs

\begin{tabular}{|c|c|c|c|c|}
\hline \multirow[b]{3}{*}{ Variable } & \multicolumn{4}{|c|}{ DV: Prescription drug costs } \\
\hline & \multicolumn{2}{|l|}{ Part $1^{\text {a }}$} & \multicolumn{2}{|l|}{ Part $2^{\mathrm{b}}$} \\
\hline & $\begin{array}{l}\text { Odds Ratio } \\
(S E)\end{array}$ & $\begin{array}{c}p \\
\text { value }\end{array}$ & $\begin{array}{l}\beta \text { coefficient } \\
(S E)\end{array}$ & $\begin{array}{c}p \\
\text { value }\end{array}$ \\
\hline \multicolumn{5}{|l|}{ COPD (Ref: No) } \\
\hline Yes & $2.271(0.360)$ & $0.000 *$ & $0.206(0.018)$ & $0.000^{*}$ \\
\hline \multicolumn{5}{|l|}{ Demographic } \\
\hline \multicolumn{5}{|l|}{ Race (Ref: non-White) } \\
\hline White & $1.246(0.301)$ & 0.364 & $0.186(0.039)$ & $0.000^{*}$ \\
\hline \multicolumn{4}{|l|}{ County_Smokerate(Re } & \\
\hline$>=31 \%$ & $1.440(0.239)$ & $0.028 *$ & $0.087(0.026)$ & $0.001 *$ \\
\hline
\end{tabular}

\section{Overall comorbidity}

$\begin{array}{rllll}\text { D'Hoore-CCI } & 1.128(0.058) & 0.019^{*} & 0.108(0.005) & 0.000^{*} \\ \begin{array}{r}\text { Number of other } \\ \text { chronic conditions }\end{array} & 2.020(0.153) & 0.000^{*} & 0.086(0.007) & 0.000^{*}\end{array}$

$* \mathrm{p}<0.05$

${ }^{a}$ Part 1: Logistic regression: $-2 \log$ likelihood $=-1099.56, \chi^{2}=238.82, p=0.000$; Pseudo $\mathrm{R}^{2}=9.80 \%$

${ }^{\mathrm{b}}$ Part 2: GLM family (gamma) $\operatorname{link}(\log )$ : AIC $=18.70$ Deviance=10514.38; $(1 / \mathrm{df})$ Deviance $=0.87$ 
TABLE A13: [KY Medicaid] Two-part model of the impact of COPD on nursing home costs

\begin{tabular}{|c|c|c|c|c|}
\hline \multirow[b]{3}{*}{ Variable } & \multicolumn{4}{|c|}{ DV: Nursing home costs } \\
\hline & \multicolumn{2}{|l|}{ Part $1^{\mathrm{a}}$} & \multicolumn{2}{|l|}{ Part $2^{\mathrm{b}}$} \\
\hline & $\begin{array}{l}\text { Odds Ratio } \\
(S E)\end{array}$ & $\begin{array}{c}p \\
\text { value }\end{array}$ & $\begin{array}{l}\beta \text { coefficient } \\
(S E)\end{array}$ & $\begin{array}{c}p \\
\text { value }\end{array}$ \\
\hline \multicolumn{5}{|l|}{ COPD (Ref: No) } \\
\hline Yes & $0.916(0.098)$ & 0.414 & $-0.524(0.127)$ & $0.000^{*}$ \\
\hline \multicolumn{5}{|l|}{ Demographic } \\
\hline \multicolumn{5}{|l|}{ Race (Ref: non-White) } \\
\hline White & $0.780(0.157)$ & 0.218 & $-0.662(0.245)$ & $0.007 *$ \\
\hline \multicolumn{4}{|l|}{ County_Smokerate(Re } & \\
\hline$>=31 \%$ & $0.918(0.133)$ & 0.557 & $0.024(0.168)$ & 0.888 \\
\hline
\end{tabular}

\section{Overall comorbidity}

$\begin{array}{rllll}\text { D'Hoore-CCI } & 1.271(0.023) & 0.000^{*} & -0.043(0.021) & 0.046^{*} \\ \begin{array}{r}\text { Number of other } \\ \text { chronic conditions }\end{array} & 0.833(0.034) & 0.000 * & -0.157(0.048) & 0.001 *\end{array}$

$* \mathrm{p}<0.05$

${ }^{a}$ Part 1: Logistic regression: $-2 \log$ likelihood $=-1759.62, \chi^{2}=147.86, \mathrm{p}=0.000$; Pseudo $\mathrm{R}^{2}=4.03 \%$

${ }^{\mathrm{b}}$ Part 2: Semi- $\log$ OLS: $\mathrm{R}^{2}=20.8 \% ; \mathrm{p}=0.000$; Test for heteroskedasticity $\left(\chi^{2}=17.27, \mathrm{p}=0.436\right)$ 
TABLE A14: [KY Medicaid] Generalized linear model (GLM) of the impact of COPD on total healthcare costs

\begin{tabular}{|c|c|c|}
\hline \multirow[b]{2}{*}{ Variable } & \multicolumn{2}{|c|}{ DV: Total healthcare costs } \\
\hline & $\begin{array}{l}\beta \text { coefficient } \\
(S E)\end{array}$ & $\begin{array}{c}p \\
\text { value }\end{array}$ \\
\hline \multicolumn{3}{|l|}{ COPD (Ref: No) } \\
\hline Yes & $0.426(0.017)$ & $0.000^{*}$ \\
\hline \multicolumn{3}{|l|}{ Demographic } \\
\hline \multicolumn{3}{|l|}{ Race (Ref: non-White) } \\
\hline White & $0.000(0.038)$ & 0.992 \\
\hline \multicolumn{3}{|l|}{ County_Smokerate(Ref: } \\
\hline$>=31 \%$ & $-0.069(0.026)$ & $0.006^{*}$ \\
\hline
\end{tabular}

\section{Overall comorbidity}

\begin{tabular}{rcc} 
D'Hoore-CCI & $0.207(0.005)$ & $0.000 *$ \\
$\begin{array}{r}\text { Number of other chronic } \\
\text { conditions }^{\mathrm{a}}\end{array}$ & $0.122(0.007)$ & $0.000 *$ \\
\hline * $<0.05$ \\
GLM [family (gamma) link(log)]: AIC $=20.37$; Deviance $=9161.44 ;(1 / \mathrm{df})$ Deviance $=0.77$
\end{tabular}


TABLE A15: [WV Medicaid] Two-part model of the impact of asthma on COPD-related hospitalization costs

\begin{tabular}{|c|c|c|c|c|}
\hline \multirow[b]{3}{*}{ Variable } & \multicolumn{4}{|c|}{ DV: COPD-related hospitalization costs } \\
\hline & \multicolumn{2}{|l|}{ Part $1^{\mathrm{a}}$} & \multicolumn{2}{|l|}{ Part $2^{\mathrm{b}}$} \\
\hline & $\begin{array}{l}\text { Odds Ratio } \\
(S E)\end{array}$ & $\begin{array}{c}p \\
\text { value }\end{array}$ & $\begin{array}{l}\beta \text { coefficient } \\
(S E)\end{array}$ & $\begin{array}{c}p \\
\text { value }\end{array}$ \\
\hline \multicolumn{5}{|l|}{ Concomitant asthma (Ref: No) } \\
\hline Yes & $1.561(0.130)$ & $0.000^{*}$ & $0.057(0.038)$ & 0.140 \\
\hline \multicolumn{5}{|l|}{ Demographic } \\
\hline Age (years) & $1.012(0.005)$ & $0.010^{*}$ & $-0.001(0.002)$ & 0.720 \\
\hline \multicolumn{5}{|l|}{ Gender (Ref: Male) } \\
\hline Female & $1.100(0.079)$ & 0.184 & $-0.011(0.036)$ & 0.753 \\
\hline \multicolumn{5}{|l|}{ Race (Ref: non-White) } \\
\hline White & $0.850(0.153)$ & 0.367 & $0.058(0.086)$ & 0.502 \\
\hline County_Smokerate(Ref: $<31 \%)$ & & & & \\
\hline$>=31 \%$ & $0.771(0.063)$ & $0.002 *$ & $-0.013(0.041)$ & 0.757 \\
\hline
\end{tabular}

\section{Overall comorbidity}

$$
\text { D’Hoore-CCI }^{\mathrm{a}} \quad 1.071(0.018) \quad 0.000 * \quad 0.017(0.007) \quad 0.025^{*}
$$

Number of other chronic conditions ${ }^{\mathrm{b}} \quad 1.223(0.032) \quad 0.000^{*} \quad 0.042(0.013) \quad 0.001^{*}$

\section{COPD-related comorbidity}

Presence of upper respiratory tract infections ${ }^{c}$

$1.074(0.076) \quad 0.313 \quad 0.033(0.035) \quad 0.346$

Presence of lower respiratory tract infections ${ }^{\mathrm{d}}$

$3.175(0.263) \quad 0.000^{*} \quad 0.225(0.036) \quad 0.000 *$

Presence of septicemia

$0.567(0.141) \quad 0.023 * \quad-0.079(0.098)$

0.421

Presence of allergic rhinitis

$0.762(0.082) \quad 0.012$

$-0.091(0.055)$

0.097

Presence of other diseases of the lung ${ }^{\mathrm{e}}$

$$
2.060(0.155) \quad 0.000 *
$$

$0.316(0.036)$

$0.000^{*}$

Presence of congestive heart failure

$1.485(0.124) \quad 0.000^{*}$

$0.075(0.037)$

$0.044^{*}$

Presence of disorders of fluid,

$2.009(0.170) \quad 0.000^{*}$

$0.240(0.037)$

$0.000^{*}$ electrolyte, and acid-base balance

$* \mathrm{p}<0.05$

${ }^{a}$ Part 1: Logistic regression: $-2 \log$ likelihood $=-2616.02 ; \chi^{2}=1074.59 ; \mathrm{p}=0.000 ;$ Pseudo $\mathrm{R}^{2}=17.04 \%$

${ }^{b}$ Part 2: Semi-log OLS: $R^{2}=17.00 \% ; p=0.000$; Test for heteroskedasticity $\left(\chi^{2}=65.51, p=0.999\right)$ 
TABLE A16: [WV Medicaid] Two-part model of the impact of asthma on COPD-related ER costs

\begin{tabular}{|c|c|c|c|c|}
\hline \multirow[b]{3}{*}{ Variable } & \multicolumn{4}{|c|}{ DV: COPD-related ER costs } \\
\hline & \multicolumn{2}{|l|}{ Part $1^{\mathrm{a}}$} & \multicolumn{2}{|l|}{ Part $2^{b}$} \\
\hline & $\begin{array}{l}\text { Odds Ratio } \\
(S E)\end{array}$ & $\begin{array}{c}p \\
\text { value }\end{array}$ & $\begin{array}{l}\beta \text { coefficient } \\
(S E)\end{array}$ & $\begin{array}{c}p \\
\text { value }\end{array}$ \\
\hline \multicolumn{5}{|l|}{ Concomitant asthma (Ref: No) } \\
\hline Yes & $1.608(0.124)$ & $0.000^{*}$ & $0.109(0.059)$ & 0.083 \\
\hline \multicolumn{5}{|l|}{ Demographic } \\
\hline Age (years) & $0.992(0.004)$ & 0.050 & $-0.009(0.003)$ & $0.008 *$ \\
\hline \multicolumn{5}{|l|}{ Gender (Ref: Male) } \\
\hline Female & $0.865(0.059)$ & $0.032 *$ & $-0.052(0.054)$ & 0.342 \\
\hline \multicolumn{5}{|l|}{ Race (Ref: non-White) } \\
\hline White & $1.009(0.173)$ & 0.957 & $-0.051(0.138)$ & 0.709 \\
\hline County_Smokerate(Ref: $<31 \%)$ & & & & \\
\hline$>=31 \%$ & $1.067(0.079)$ & 0.386 & $0.086(0.060)$ & 0.147 \\
\hline
\end{tabular}

\section{Overall comorbidity}

$$
\begin{array}{llll}
\text { D’Hoore-CCI }^{\mathrm{a}} \quad 0.989(0.016) & 0.506 & 0.008(0.013) & 0.536
\end{array}
$$

Number of other chronic conditions ${ }^{\mathrm{b}} \quad 1.118(0.028) \quad 0.000^{*} \quad 0.118(0.021) \quad 0.000^{*}$

\section{COPD-related comorbidity}

Presence of upper respiratory tract infections ${ }^{c}$

Presence of lower respiratory tract infections $^{\mathrm{d}}$

Presence of septicemia

Presence of allergic rhinitis

Presence of other diseases of the lung ${ }^{\mathrm{e}}$

Presence of congestive heart failure

Presence of disorders of fluid, electrolyte, and acid-base balance
$1.762(0.119) \quad 0.000 * \quad 0.065(0.056) \quad 0.244$

$1.437(0.117) \quad 0.000 *$

$0.112(0.061)$

0.066

$$
0.742(0.174) \quad 0.203 \quad-0.025(0.175) \quad 0.887
$$

$$
0.779(0.079) \quad 0.014^{*} \quad 0.009(0.081) \quad 0.916
$$

$$
1.688(0.125) \quad 0.000 * \quad 0.239(0.057) \quad 0.000 *
$$

$$
1.120(0.092) \quad 0.166^{*}
$$

$0.081(0.064)$

0.210

$1.578(0.130) \quad 0.000^{*}$

$0.291(0.062)$

$0.000^{*}$

$* \mathrm{p}<0.05$

${ }^{a}$ Part 1: Logistic regression: $-2 \operatorname{loglikelihood}=-2863.66 ; \chi^{2}=416.91 ; \mathrm{p}=0.000 ;$ Pseudo $\mathrm{R}^{2}=6.79 \%$

${ }^{\mathrm{b}}$ Part 2: Semi-log OLS: $\mathrm{R}^{2}=9.59 \%$; $=0.000$; Test for heteroskedasticity $\left(\chi^{2}=111.81, \mathrm{p}=0.382\right)$ 
TABLE A17: [WV Medicaid] Two-part model of the impact of asthma on COPD-related outpatient costs

\begin{tabular}{|c|c|c|c|c|}
\hline \multirow[b]{3}{*}{ Variable } & \multicolumn{4}{|c|}{ DV: COPD-related Outpatient costs } \\
\hline & \multicolumn{2}{|l|}{ Part $1^{\text {a }}$} & \multicolumn{2}{|l|}{ Part $2^{b}$} \\
\hline & $\begin{array}{l}\text { Odds Ratio } \\
(S E)\end{array}$ & $\begin{array}{c}p \\
\text { value }\end{array}$ & $\begin{array}{l}\beta \text { coefficient } \\
(S E)\end{array}$ & $\begin{array}{c}p \\
\text { value }\end{array}$ \\
\hline \multicolumn{5}{|l|}{ Concomitant asthma (Ref: No) } \\
\hline Yes & $0.888(0.116)$ & 0.362 & $0.084(0.061)$ & 0.173 \\
\hline \multicolumn{5}{|l|}{ Demographic } \\
\hline Age (years) & $1.022(0.007)$ & $0.001 *$ & $-0.002(0.003)$ & 0.614 \\
\hline \multicolumn{5}{|l|}{ Gender (Ref: Male) } \\
\hline Female & $1.085(0.120)$ & 0.461 & $0.031(0.050)$ & 0.540 \\
\hline \multicolumn{5}{|l|}{ Race (Ref: non-White) } \\
\hline White & $1.382(0.340)$ & 0.189 & $0.288(0.131)$ & $0.029 *$ \\
\hline $\begin{array}{r}\text { County_Smokerate(Ref: }<31 \%) \\
>=31 \%\end{array}$ & $0.843(0.100)$ & 0.150 & $0.084(0.056)$ & 0.137 \\
\hline
\end{tabular}

Overall comorbidity

$\begin{array}{llll}\text { D’Hoore-CCI }^{\mathrm{a}} \quad 0.960(0.026) & 0.126 & 0.015(0.012) & 0.226\end{array}$

Number of other chronic conditions $^{\mathrm{b}} \quad 1.022(0.042) \quad 0.600 \quad 0.067(0.019) \quad 0.000^{*}$

\section{COPD-related comorbidity}

Presence of upper respiratory tract infections $^{c}$

$0.735(0.082) \quad 0.006^{*} \quad 0.144(0.050) \quad 0.004^{*}$

Presence of lower respiratory tract infections $^{\mathrm{d}}$

$1.310(0.203) \quad 0.081 \quad 0.317(0.064) \quad 0.000 *$

Presence of septicemia

$0.600(0.216) \quad 0.156$

$-0.016(0.186)$

0.930

Presence of allergic rhinitis

$1.887(0.370) \quad 0.001^{*}$

$0.125(0.074)$

0.091

Presence of other diseases of the lung ${ }^{\mathrm{e}}$

$1.465(0.202) \quad 0.006^{*}$

$0.404(0.058)$

$0.000^{*}$

Presence of congestive heart failure

$$
1.419(0.219) \quad 0.023 *
$$

$0.523(0.063)$

$0.000^{*}$

Presence of disorders of fluid,

$0.885(0.128) \quad 0.401$

$0.142(0.065)$

$0.030 *$ electrolyte, and acid-base balance

$* \mathrm{p}<0.05$

${ }^{a}$ Part 1: Logistic regression: $-2 \log$ likelihood $=-1344.62 ; \chi^{2}=63.29 ; \mathrm{p}=0.000 ;$ Pseudo $\mathrm{R}^{2}=2.30 \%$

${ }^{\mathrm{b}}$ Part 2: Semi-log OLS: $\mathrm{R}^{2}=8.25 \%$; $\mathrm{p}=0.000$; Test for heteroskedasticity $\left(\chi^{2}=123.16, \mathrm{p}=0.151\right)$ 
TABLE A18: [WV Medicaid] Two-part model of the impact of asthma on COPD-related physician costs

\begin{tabular}{|c|c|c|c|c|}
\hline \multirow[b]{3}{*}{ Variable } & \multicolumn{4}{|c|}{ DV: COPD-related Physician costs } \\
\hline & \multicolumn{2}{|l|}{ Part $1^{\text {a }}$} & \multicolumn{2}{|l|}{ Part $2^{\mathrm{b}}$} \\
\hline & $\begin{array}{l}\text { Odds Ratio } \\
(S E)\end{array}$ & $\begin{array}{c}p \\
\text { value }\end{array}$ & $\begin{array}{l}\beta \text { coefficient } \\
(S E)\end{array}$ & $\begin{array}{c}p \\
\text { value }\end{array}$ \\
\hline \multicolumn{5}{|l|}{ Concomitant asthma (Ref: No) } \\
\hline Yes & $1.079(0.082)$ & 0.315 & $0.062(0.042)$ & 0.147 \\
\hline \multicolumn{5}{|l|}{ Demographic } \\
\hline Age (years) & $1.007(0.004)$ & 0.061 & $-0.011(0.002)$ & $0.000 *$ \\
\hline \multicolumn{5}{|l|}{ Gender (Ref: Male) } \\
\hline Female & $1.008(0.062)$ & 0.899 & $-0.061(0.037)$ & 0.094 \\
\hline \multicolumn{5}{|l|}{ Race (Ref: non-White) } \\
\hline White & $1.234(0.192)$ & 0.177 & $0.349(0.097)$ & $0.000 *$ \\
\hline County_Smokerate(Ref: $<31 \%$ ) & & & & \\
\hline$>=31 \%$ & $0.885(0.061)$ & 0.077 & $0.047(0.040)$ & 0.250 \\
\hline
\end{tabular}

Overall comorbidity

D’Hoore-CCI $^{\mathrm{a}} \quad 0.960(0.015) \quad 0.005^{*} \quad 0.034(0.009) \quad 0.000 *$

Number of other chronic conditions ${ }^{\mathrm{b}} \quad 1.089(0.025) \quad 0.000^{*} \quad 0.098(0.014) \quad 0.000^{*}$

\section{COPD-related comorbidity}

Presence of upper respiratory tract infections ${ }^{c}$

Presence of lower respiratory tract infections $^{\mathrm{d}}$

Presence of septicemia

Presence of allergic rhinitis

Presence of other diseases of the lung ${ }^{\mathrm{e}}$

Presence of congestive heart failure

Presence of disorders of fluid, electrolyte, and acid-base balance $\begin{array}{llll}1.062(0.066) & 0.333 & 0.070(0.036) & 0.050\end{array}$

$1.214(0.098) \quad 0.017 * \quad 0.146(0.046) \quad 0.001 *$

$\begin{array}{llll}0.676(0.148) & 0.073 & -0.192(0.139) & 0.166\end{array}$

$1.268(0.122) \quad 0.013^{*}$

$0.185(0.051) \quad 0.000 *$

$1.140(0.083) \quad 0.072 \quad 0.134(0.041) \quad 0.001^{*}$

$0.911(0.071) \quad 0.231 \quad 0.146(0.046) \quad 0.002 *$

$\begin{array}{llll}0.869(0.070) & 0.080 & 0.053(0.047) & 0.265\end{array}$

$* \mathrm{p}<0.05$

${ }^{a}$ Part 1: Logistic regression: $-2 \log$ likelihood $=-3232.30 ; \chi^{2}=57.14 ; p=0.000$; Pseudo $\mathrm{R}^{2}=0.88 \%$

${ }^{\mathrm{b}}$ Part 2: GLM family (gamma) link $(\log ):$ AIC $=12.80$ Deviance=2103.94; $(1 / \mathrm{df})$ Deviance $=0.80$ 
TABLE A19: [WV Medicaid] Two-part model of the impact of asthma on COPD-related prescription drug costs

\begin{tabular}{|c|c|c|c|c|}
\hline \multirow[b]{3}{*}{ Variable } & \multicolumn{4}{|c|}{ DV: COPD-related Prescription drug costs } \\
\hline & \multicolumn{2}{|l|}{ Part $1^{\text {a }}$} & \multicolumn{2}{|l|}{ Part $2^{\mathrm{b}}$} \\
\hline & $\begin{array}{l}\text { Odds Ratio } \\
(S E)\end{array}$ & $\begin{array}{c}p \\
\text { value }\end{array}$ & $\begin{array}{l}\beta \text { coefficient } \\
(S E)\end{array}$ & $\begin{array}{c}p \\
\text { value }\end{array}$ \\
\hline \multicolumn{5}{|l|}{ Concomitant asthma (Ref: No) } \\
\hline Yes & $1.279(0.183)$ & 0.085 & $0.376(0.040)$ & $0.000^{*}$ \\
\hline \multicolumn{5}{|l|}{ Demographic } \\
\hline Age (years) & $0.954(0.006)$ & $0.000 *$ & $0.017(0.002)$ & $0.000 *$ \\
\hline \multicolumn{5}{|l|}{ Gender (Ref: Male) } \\
\hline Female & $1.493(0.147)$ & $0.000 *$ & $-0.025(0.034)$ & 0.456 \\
\hline \multicolumn{5}{|l|}{ Race (Ref: non-White) } \\
\hline White & $1.590(0.370)$ & $0.046^{*}$ & $0.461(0.088)$ & $0.000 *$ \\
\hline County_Smokerate(Ref: $<31 \%)$ & & & & \\
\hline$>=31 \%$ & $1.440(0.179)$ & $0.003 *$ & $0.042(0.037)$ & 0.255 \\
\hline
\end{tabular}

\section{Overall comorbidity}

$$
\text { D’Hoore-CCI }^{\mathrm{a}} \quad 1.014(0.027) \quad 0.587 \quad-0.034(0.008) \quad 0.000^{*}
$$

Number of other chronic conditions ${ }^{\mathrm{b}} \quad 1.239(0.049) \quad 0.000^{*} \quad-0.045(0.013) \quad 0.000^{*}$

\section{COPD-related comorbidity}

Presence of upper respiratory tract infections ${ }^{c}$

Presence of lower respiratory tract infections $^{\mathrm{d}}$

Presence of septicemia

Presence of allergic rhinitis

Presence of other diseases of the lung ${ }^{\mathrm{e}}$

Presence of congestive heart failure

Presence of disorders of fluid, electrolyte, and acid-base balance
$1.976(0.207) \quad 0.000 * \quad 0.112(0.034) \quad 0.001^{*}$

$1.024(0.140) \quad 0.862 \quad 0.285(0.043) \quad 0.000 *$

$1.344(0.599) \quad 0.508 \quad 0.043(0.119) \quad 0.719$

$1.062(0.181) \quad 0.722 \quad 0.108(0.050) \quad 0.031^{*}$

$1.137(0.139) \quad 0.293 \quad 0.189(0.038) \quad 0.000^{*}$

$1.102(0.147) \quad 0.465 \quad 0.112(0.042) \quad 0.007^{*}$

$1.191(0.172) \quad 0.229 \quad 0.028(0.043) \quad 0.514$

$* \mathrm{p}<0.05$

${ }^{a}$ Part 1: Logistic regression: $-2 \operatorname{loglikelihood}=-1518.54 ; \chi^{2}=241.23 ; \mathrm{p}=0.000 ;$ Pseudo $\mathrm{R}^{2}=7.36 \%$

${ }^{\mathrm{b}}$ Part 2: GLM family (gamma) link $(\log ): \mathrm{AIC}=15.76$ Deviance $=5085.86(1 / \mathrm{df})$ Deviance $=1.14$ 
TABLE A20: [WV Medicaid] Semi-log OLS model of the impact of asthma on total COPD-related costs

\begin{tabular}{|c|c|c|}
\hline \multirow[b]{2}{*}{ Variable } & \multicolumn{2}{|c|}{ DV: Total COPD-related costs } \\
\hline & $\begin{array}{l}\beta \text { coefficient } \\
(S E)\end{array}$ & $\begin{array}{c}p \\
\text { value }\end{array}$ \\
\hline \multicolumn{3}{|l|}{ Concomitant asthma (Ref: No) } \\
\hline 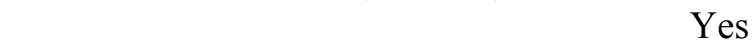 & $0.392(0.045)$ & $0.000^{*}$ \\
\hline \multicolumn{3}{|l|}{ Demographic } \\
\hline Age (years) & $0.006(0.002)$ & $0.018^{*}$ \\
\hline Gender (Ref: Male) & & \\
\hline Female & $0.061(0.037)$ & 0.102 \\
\hline Race (Ref: non-White) & & \\
\hline White & $0.196(0.096)$ & $0.042 *$ \\
\hline County_Smokerate(Ref: $<31 \%)$ & & \\
\hline$>=31 \%$ & $-0.033(0.042)$ & 0.430 \\
\hline
\end{tabular}

\section{Overall comorbidity}

$\begin{array}{rrr}\text { D'Hoore-CCI }^{\mathrm{a}} & 0.034(0.009) & 0.000^{*} \\ \text { Number of other chronic conditions }^{\mathrm{b}} & 0.101(0.014) & 0.000^{*}\end{array}$

\section{COPD-related comorbidity}

$$
\begin{aligned}
& \text { Presence of upper respiratory tract infections }{ }^{c} \quad 0.149(0.037) \quad 0.000^{*} \\
& \text { Presence of lower respiratory tract infections }{ }^{\mathrm{d}} \quad 0.673(0.048) \quad 0.000^{*} \\
& \text { Presence of septicemia } \quad-0.345(0.156) \quad 0.011^{*} \\
& \text { Presence of allergic rhinitis } \quad-0.007(0.056) \quad 0.905 \\
& \text { Presence of other diseases of the lung }{ }^{\mathrm{e}} \quad 0.530(0.043) \quad 0.000^{*} \\
& \text { Presence of congestive heart failure } 0.351(0.047) \quad 0.000 *
\end{aligned}
$$

$* \mathrm{p}<0.05$

Semi-log OLS: $\mathrm{R}^{2}=23.82 \%$; $=0.000$; Test for heteroskedasticity $\left(\chi^{2}=0.64, \mathrm{p}=0.423\right)$ 
TABLE A21: [KY Medicaid] Two-part model of the impact of asthma on COPD-related hospitalization costs

\begin{tabular}{|c|c|c|c|c|}
\hline \multirow[b]{3}{*}{ Variable } & \multicolumn{4}{|c|}{ DV: COPD-related hospitalization costs } \\
\hline & \multicolumn{2}{|l|}{ Part $1^{\mathrm{a}}$} & \multicolumn{2}{|l|}{ Part $2^{\mathrm{b}}$} \\
\hline & $\begin{array}{l}\text { Odds Ratio } \\
(S E)\end{array}$ & $\begin{array}{c}p \\
\text { value }\end{array}$ & $\begin{array}{l}\beta \text { coefficient } \\
(S E)\end{array}$ & $\begin{array}{c}p \\
\text { value }\end{array}$ \\
\hline \multicolumn{5}{|l|}{ Concomitant asthma (Ref: No) } \\
\hline Yes & $1.633(0.122)$ & $0.000 *$ & $0.155(0.042)$ & $0.000^{*}$ \\
\hline \multicolumn{5}{|l|}{ Demographic } \\
\hline Age (years) & $1.008(0.004)$ & $0.048 *$ & $0.009(0.003)$ & $0.001 *$ \\
\hline \multicolumn{5}{|l|}{ Gender (Ref: Male) } \\
\hline Female & $1.007(0.066)$ & 0.913 & $0.014(0.039)$ & 0.713 \\
\hline \multicolumn{5}{|l|}{ Race (Ref: non-White) } \\
\hline White & $1.166(0.205)$ & 0.381 & $-0.084(0.106)$ & 0.430 \\
\hline County_Smokerate(Ref: $<31 \%)$ & & & & \\
\hline$>=31 \%$ & $1.191(0.121)$ & 0.084 & $0.088(0.059)$ & 0.135 \\
\hline
\end{tabular}

\section{Overall comorbidity}

$$
\begin{array}{lllll}
\text { D'Hoore-CCI }^{\mathrm{a}} \quad 1.089(0.017) & 0.000^{*} \quad 0.025(0.008) \quad 0.002 *
\end{array}
$$

$\begin{array}{lllll}\text { Number of other chronic conditions }^{\mathrm{b}} & 1.207(0.029) & 0.000 * & 0.010(0.014) & 0.458\end{array}$

\section{COPD-related comorbidity}

Presence of upper respiratory tract infections ${ }^{c}$

$1.339(0.090) \quad 0.000^{*} \quad-0.034(0.042)$

0.413

Presence of lower respiratory tract infections $^{\mathrm{d}}$

$2.976(0.217) \quad 0.000^{*} \quad 0.244(0.039) \quad 0.000^{*}$

Presence of septicemia

$0.446(0.098)$

$0.000^{*}$

Presence of allergic rhinitis

$0.681(0.063) \quad 0.000 *$

$-0.008(0.056)$

0.887

Presence of other diseases of the lung ${ }^{\mathrm{e}}$

$2.090(0.142) \quad 0.000 *$

$0.493(0.039)$

$0.000^{*}$

Presence of congestive heart failure

$1.520(0.117) \quad 0.000 *$

$0.160(0.042)$

$0.000^{*}$

Presence of disorders of fluid,

$2.458(0.194) \quad 0.000 *$

$0.304(0.040)$

$0.000^{*}$ electrolyte, and acid-base balance

$* \mathrm{p}<0.05$

${ }^{a}$ Part 1: Logistic regression: $-2 \log$ likelihood $=-3233.46 ; \chi^{2}=1436.42 ; \mathrm{p}=0.000 ;$ Pseudo $\mathrm{R}^{2}=18.17 \%$

${ }^{\mathrm{b}}$ Part 2: Semi-log OLS: $\mathrm{R}^{2}=22.96 \% ; \mathrm{p}=0.000$; Test for heteroskedasticity $\left(\chi^{2}=112.26, \mathrm{p}=0.370\right)$ 
TABLE A22: [KY Medicaid] Two-part model of the impact of asthma on COPD-related ER costs

$$
\text { DV: COPD-related ER costs }
$$

Variable

\begin{tabular}{lclll}
${\text { Part } 1^{\mathrm{a}}}$ & & & ${\text { Part } 2^{\mathrm{b}}}$ & \\
\cline { 1 - 2 } $\begin{array}{l}\text { Odds Ratio } \\
(S E)\end{array}$ & $p$ & & $\beta$ coefficient & $p$ \\
value & & $(S E)$ & value
\end{tabular}

Concomitant asthma (Ref: No)

$\begin{array}{llll}\text { Yes } & 1.448(0.101) & 0.000^{*} & 0.197(0.047) \quad 0.000^{*}\end{array}$

Demographic

$\begin{array}{rllll}\text { Age (years) } & 0.989(0.004) & 0.005^{*} & 0.001(0.003) & 0.679 \\ \text { Gender (Ref: Male) } & & & & \\ \text { Female } & 1.044(0.065) & 0.487 & -0.132(0.045) & 0.003 * \\ \text { Race (Ref: non-White) } & & & & \\ \text { White } & 0.896(0.144) & 0.494 & 0.100(0.113) & 0.374 \\ \text { County_Smokerate(Ref: }<31 \%) & & & & \\ >=31 \% & 0.788(0.072) & 0.009^{*} & 0.116(0.062) & 0.062\end{array}$

Overall comorbidity

$\begin{array}{lllll}\text { D’Hoore-CCI }^{\mathrm{a}} \quad 0.994(0.015) & 0.689 & 0.017(0.010) & 0.096\end{array}$

Number of other chronic conditions ${ }^{\mathrm{b}} \quad 1.062(0.024) \quad 0.008^{*} \quad 0.113(0.016) \quad 0.000^{*}$

\section{COPD-related comorbidity}

Presence of upper respiratory tract

$2.037(0.135) \quad 0.000^{*} \quad-0.019(0.050)$

0.699

infections ${ }^{c}$

Presence of lower respiratory tract

infections ${ }^{\mathrm{d}}$

$1.311(0.094) \quad 0.000^{*} \quad 0.129(0.048) \quad 0.007 *$

Presence of septicemia

$0.856(0.168) \quad 0.429$

$-0.133(0.130)$

0.304

Presence of allergic rhinitis

$0.806(0.069) \quad 0.011 *$

$-0.046(0.061)$

0.449

Presence of other diseases of the lung ${ }^{\mathrm{e}}$

$1.621(0.108) \quad 0.000 *$

$0.141(0.045)$

$0.002 *$

Presence of congestive heart failure

$1.144(0.086) \quad 0.073$

$0.085(0.052)$

0.102

Presence of disorders of fluid,

$1.440(0.110) \quad 0.000 *$

$0.235(0.051)$

$0.000^{*}$ electrolyte, and acid-base balance

$* \mathrm{p}<0.05$

${ }^{a}$ Part 1: Logistic regression: $-2 \log$ likelihood $=-3517.78 ; \chi^{2}=465.49 ; \mathrm{p}=0.000 ;$ Pseudo $\mathrm{R}^{2}=6.21 \%$

${ }^{\mathrm{b}}$ Part 2: Semi-log OLS: $\mathrm{R}^{2}=10.91 \% ; \mathrm{p}=0.000$; Test for heteroskedasticity $\left(\chi^{2}=152.69, \mathrm{p}=0.003\right)$ Semi-log OLS model was used because GLM model was not a good fit. 
TABLE A23: [KY Medicaid] Two-part model of the impact of asthma on COPD-related Outpatient costs

\begin{tabular}{|c|c|c|c|c|}
\hline \multirow[b]{3}{*}{ Variable } & \multicolumn{4}{|c|}{ DV: COPD-related outpatient costs } \\
\hline & \multicolumn{2}{|l|}{ Part $1^{\text {a }}$} & \multicolumn{2}{|l|}{ Part $2^{\mathrm{b}}$} \\
\hline & $\begin{array}{l}\text { Odds Ratio } \\
(S E)\end{array}$ & $\begin{array}{c}p \\
\text { value }\end{array}$ & $\begin{array}{l}\beta \text { coefficient } \\
\text { (SE) }\end{array}$ & $\begin{array}{c}p \\
\text { value }\end{array}$ \\
\hline \multicolumn{5}{|l|}{ Concomitant asthma (Ref: No) } \\
\hline$x^{2}$ & $1.045(0.093)$ & 0.619 & $0.223(0.052)$ & $0.019^{*}$ \\
\hline \multicolumn{5}{|l|}{ Demographic } \\
\hline Age (years) & $1.031(0.005)$ & $0.000 *$ & $0.038(0.003)$ & $0.000^{*}$ \\
\hline \multicolumn{5}{|l|}{ Gender (Ref: Male) } \\
\hline Female & $0.796(0.060)$ & $0.003 *$ & $0.244 \quad(0.043)$ & $0.000 *$ \\
\hline \multicolumn{5}{|l|}{ Race (Ref: non-White) } \\
\hline White & $1.254(0.229)$ & 0.214 & $0.366(0.117)$ & $0.002 *$ \\
\hline County_Smokerate(Ref: $<31 \%$ ) & & & & \\
\hline$>=31 \%$ & $1.075(0.121)$ & 0.522 & $-0.109(0.066)$ & 0.100 \\
\hline
\end{tabular}

\section{Overall comorbidity}

$$
\text { D’Hoore-CCI }^{\mathrm{a}} \quad 0.925(0.016) \quad 0.000 * \quad 0.070(0.011) \quad 0.000 *
$$

Number of other chronic conditions $^{\mathrm{b}} \quad 1.003(0.028) \quad 0.901 \quad-0.017(0.016) \quad 0.000^{*}$

\section{COPD-related comorbidity}

Presence of upper respiratory tract infections ${ }^{c}$

Presence of lower respiratory tract infections ${ }^{\mathrm{d}}$

Presence of septicemia

Presence of allergic rhinitis

Presence of other diseases of the lung

Presence of congestive heart failure

Presence of disorders of fluid, electrolyte, and acid-base balance
$1.091(0.082) \quad 0.251 \quad-0.159(0.045) \quad 0.000^{*}$

$1.633(0.163) \quad 0.000 * \quad 0.452(0.052) \quad 0.000 *$

$0.757(0.179) \quad 0.240 \quad 0.116(0.151) \quad 0.442$

$1.343(0.148) \quad 0.008 * \quad-0.130(0.060) \quad 0.029 *$

$1.072(0.092) \quad 0.418 \quad 0.534(0.049) \quad 0.000^{*}$

$0.873(0.080) \quad 0.140 \quad 0.574(0.054) \quad 0.000^{*}$

$1.053(0.104) \quad 0.601 \quad 0.211(0.057) \quad 0.000^{*}$

$* \mathrm{p}<0.05$

${ }^{a}$ Part 1: Logistic regression: $-2 \log$ likelihood $=-2669.95 ; \chi^{2}=110.01 ; p=0.000$; Pseudo $R^{2}=2.02 \%$

${ }^{\mathrm{b}}$ Part 2: Semi-log OLS: $\mathrm{R}^{2}=19.23 \%$; $=0.000$; Test for heteroskedasticity $\left(\chi^{2}=10.40, \mathrm{p}=0.001\right)$ Semi-log OLS model was used because GLM model was not a good fit. 
TABLE A24: [KY Medicaid] Two-part model of the impact of asthma on COPD-related Physician costs

\begin{tabular}{|c|c|c|c|c|}
\hline \multirow[b]{3}{*}{ Variable } & \multicolumn{4}{|c|}{ DV: COPD-related physician costs } \\
\hline & \multicolumn{2}{|l|}{ Part $1^{\mathrm{a}}$} & \multicolumn{2}{|l|}{ Part $2^{\mathrm{b}}$} \\
\hline & $\begin{array}{l}\text { Odds Ratio } \\
(S E)\end{array}$ & $\begin{array}{c}p \\
\text { value }\end{array}$ & $\begin{array}{l}\beta \text { coefficient } \\
(S E)\end{array}$ & $\begin{array}{c}p \\
\text { value }\end{array}$ \\
\hline \multicolumn{5}{|l|}{ Concomitant asthma (Ref: No) } \\
\hline Yes & $1.086(0.086)$ & 0.300 & $0.045(0.032)$ & 0.159 \\
\hline \multicolumn{5}{|l|}{ Demographic } \\
\hline Age (years) & $0.997(0.004)$ & 0.397 & $0.004(0.002)$ & $0.036^{*}$ \\
\hline \multicolumn{5}{|l|}{ Gender (Ref: Male) } \\
\hline Female & $0.613(0.040)$ & $0.000^{*}$ & $-0.012(0.026)$ & 0.641 \\
\hline \multicolumn{5}{|l|}{ Race (Ref: non-White) } \\
\hline White & $1.435(0.228)$ & $0.023^{*}$ & $0.201(0.075)$ & 0.054 \\
\hline County_Smokerate(Ref: <31\%) & & & & \\
\hline$>=31 \%$ & $1.101(0.105)$ & 0.313 & $-0.031(0.042)$ & 0.459 \\
\hline
\end{tabular}

Overall comorbidity

D’Hoore-CCI $^{\mathrm{a}} \quad 0.922(0.014) \quad 0.000 * \quad 0.029(0.007) \quad 0.000 *$

Number of other chronic conditions ${ }^{\mathrm{b}} \quad 1.263(0.032) \quad 0.000^{*} \quad 0.058(0.010) \quad 0.000^{*}$

\section{COPD-related comorbidity}

Presence of upper respiratory tract infections ${ }^{c}$

$1.745(0.113) \quad 0.000 * \quad 0.158(0.028) \quad 0.000 *$

Presence of lower respiratory tract infections ${ }^{\mathrm{d}}$

$1.235(0.101) \quad 0.010^{*} \quad 0.129(0.033) \quad 0.000^{*}$

Presence of septicemia

$0.388(0.075) \quad 0.000^{*}$

$0.184(0.111) \quad 0.097$

Presence of allergic rhinitis

$1.747(0.183) \quad 0.000^{*}$

$0.122(0.035)$

$0.001^{*}$

Presence of other diseases of the lung ${ }^{\mathrm{e}}$

$1.096(0.082) \quad 0.225$

$0.295(0.030)$

$0.000^{*}$

Presence of congestive heart failure

$0.805(0.064) \quad 0.006^{*}$

$0.168(0.035) \quad 0.000^{*}$

Presence of disorders of fluid,

$0.793(0.066) \quad 0.006^{*}$

$-0.044(0.036)$

0.213 electrolyte, and acid-base balance

$* \mathrm{p}<0.05$

${ }^{a}$ Part 1: Logistic regression: $-2 \operatorname{loglikelihood}=-3272.99 ; \chi^{2}=367.78 ; \mathrm{p}=0.000 ;$ Pseudo $\mathrm{R}^{2}=5.32 \%$

${ }^{\mathrm{b}}$ Part 2: GLM family (gamma) link $(\log ): \mathrm{AIC}=13.29$ Deviance $=2206.52(1 / \mathrm{df})$ Deviance $=0.76$ 
TABLE A25: [KY Medicaid] Two-part model of the impact of asthma on COPD-related Prescription drug costs

\begin{tabular}{|c|c|c|c|c|}
\hline \multirow[b]{3}{*}{ Variable } & \multicolumn{4}{|c|}{ DV: COPD-related prescription drug costs } \\
\hline & \multicolumn{2}{|l|}{ Part $1^{\text {a }}$} & \multicolumn{2}{|l|}{ Part $2^{\mathrm{b}}$} \\
\hline & $\begin{array}{l}\text { Odds Ratio } \\
(S E)\end{array}$ & $\begin{array}{c}p \\
\text { value }\end{array}$ & $\begin{array}{l}\beta \text { coefficient } \\
(S E)\end{array}$ & $\begin{array}{c}p \\
\text { value }\end{array}$ \\
\hline \multicolumn{5}{|l|}{ Concomitant asthma (Ref: No) } \\
\hline Yes & $2.470(0.758)$ & $0.003 *$ & $0.397(0.033)$ & $0.000 *$ \\
\hline \multicolumn{5}{|l|}{ Demographic } \\
\hline Age (years) & $1.006(0.010)$ & 0.498 & $0.019(0.002)$ & $0.000^{*}$ \\
\hline \multicolumn{5}{|l|}{ Gender (Ref: Male) } \\
\hline Female & $2.519(0.392)$ & $0.000 *$ & $-0.021(0.028)$ & 0.459 \\
\hline \multicolumn{5}{|l|}{ Race (Ref: non-White) } \\
\hline White & $2.013(0.647)$ & $0.029 *$ & $0.303(0.075)$ & $0.000 *$ \\
\hline County_Smokerate(Ref: $<31 \%)$ & & & & \\
\hline$>=31 \%$ & $2.005(0.382)$ & $0.000 *$ & $0.028(0.043)$ & 0.514 \\
\hline
\end{tabular}

\section{Overall comorbidity}

$$
\text { D’Hoore-CCI }^{\mathrm{a}} \quad 1.005(0.043) \quad 0.899 \quad-0.026(0.007) \quad 0.000^{*}
$$

$\begin{array}{lllll}\text { Number of other chronic conditions }^{\mathrm{b}} & 1.105(0.068) & 0.103 & -0.017(0.010) & 0.090\end{array}$

\section{COPD-related comorbidity}

Presence of upper respiratory tract infections ${ }^{c}$
$4.183(0.752) \quad 0.000^{*}$
$0.129(0.029)$
$0.000^{*}$

Presence of lower respiratory tract infections $^{\mathrm{d}}$

$1.635(0.411) \quad 0.051$

$0.170(0.034)$

$0.000 *$

Presence of septicemia

$1.178(0.742) \quad 0.794$

$-0.095(0.094)$

0.311

Presence of allergic rhinitis

$1.550(0.497) \quad 0.172$

$0.094(0.039)$

$0.016^{*}$

Presence of other diseases of the lung ${ }^{\mathrm{e}}$

$1.523(0.320) \quad 0.045^{*}$

$0.129(0.032)$

$0.000^{*}$

Presence of congestive heart failure

$0.809(0.158) \quad 0.278$

$0.112(0.035)$

$0.002 *$

Presence of disorders of fluid,

$0.829(0.187) \quad 0.405$

$-0.004(0.036)$

0.899 electrolyte, and acid-base balance

$* \mathrm{p}<0.05$

${ }^{a}$ Part 1: Logistic regression: $-2 \operatorname{loglikelihood}=-776.73 ; \chi^{2}=231.71 ; \mathrm{p}=0.000 ;$ Pseudo $\mathrm{R}^{2}=12.98 \%$

${ }^{\mathrm{b}}$ Part 2: GLM family (gamma) link $(\log ):$ AIC $=16.37$ Deviance $=6597.31(1 / \mathrm{df})$ Deviance $=1.11$ 
TABLE A26: [KY Medicaid] Semi-log OLS model of the impact of asthma on total COPD-related costs

\begin{tabular}{|c|c|c|}
\hline \multirow[b]{2}{*}{ Variable } & \multicolumn{2}{|c|}{ DV: Total COPD-related costs } \\
\hline & $\begin{array}{l}\beta \text { coefficient } \\
(S E)\end{array}$ & $\begin{array}{c}p \\
\text { value }\end{array}$ \\
\hline \multicolumn{3}{|l|}{ Concomitant asthma (Ref: No) } \\
\hline 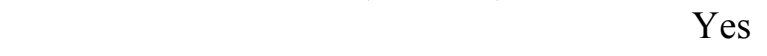 & $0.472(0.037)$ & $0.000^{*}$ \\
\hline \multicolumn{3}{|l|}{ Demographic } \\
\hline Age (years) & $0.025(0.002)$ & $0.000^{*}$ \\
\hline Gender (Ref: Male) & & \\
\hline Female & $0.127(0.031)$ & $0.000^{*}$ \\
\hline Race (Ref: non-White) & & \\
\hline White & $0.224(0.082)$ & $0.006^{*}$ \\
\hline County_Smokerate(Ref: <31\%) & & \\
\hline$>=31 \%$ & $0.021(0.047)$ & 0.661 \\
\hline
\end{tabular}

\section{Overall comorbidity}

$\begin{array}{rrr}\text { D'Hoore-CCI }^{\mathrm{a}} & 0.054(0.008) & 0.000^{*} \\ \text { Number of other chronic conditions }^{\mathrm{b}} & 0.028(0.012) & 0.017^{*}\end{array}$

\section{COPD-related comorbidity}

\begin{tabular}{|c|c|c|}
\hline Presence of upper respiratory tract infections ${ }^{c}$ & $0.026(0.032)$ & 0.408 \\
\hline Presence of lower respiratory tract infections $\mathrm{d}^{\mathrm{d}}$ & $0.498(0.038)$ & $0.000^{*}$ \\
\hline Presence of septicemia & $0.147(0.105)$ & 0.163 \\
\hline Presence of allergic rhinitis & $-0.053(0.043)$ & 0.216 \\
\hline Presence of other diseases of the lung ${ }^{\mathrm{e}}$ & $0.454(0.035)$ & $0.000^{*}$ \\
\hline Presence of congestive heart failure & $0.430(0.039)$ & $0.000^{*}$ \\
\hline $\begin{array}{r}\text { Presence of disorders of fluid, electrolyte, and } \\
\text { acid-base balance }\end{array}$ & $0.334(0.041)$ & $0.000^{*}$ \\
\hline
\end{tabular}

$* \mathrm{p}<0.05$

Semi-log OLS: $\mathrm{R}^{2}=24.89 \%$; $=0.000$; Test for heteroskedasticity $\left(\chi^{2}=2.44, \mathrm{p}=0.119\right)$ 
Appendix B: Logistic Regression Models for Obtaining Propensity Scores for Phase II - Part 2 
TABLE B1: Logistic regression predicting the probability of being prescribed ICS/LABA vs. SABD

\begin{tabular}{|c|c|c|c|}
\hline \multirow[b]{2}{*}{ Variable } & \multicolumn{3}{|c|}{ DV: Probability of receiving ICS/LABA } \\
\hline & Odds ratio $(S E)$ & $95 \% C I$ & $\begin{array}{c}p \\
\text { value }\end{array}$ \\
\hline \multicolumn{4}{|l|}{ Demographic } \\
\hline Age (years) & $0.963(0.009)$ & $0.945-0.981$ & $0.000^{*}$ \\
\hline \multicolumn{4}{|l|}{ Gender (Ref: Male) } \\
\hline Female & $1.123(0.164)$ & $0.844-1.495$ & 0.427 \\
\hline \multicolumn{4}{|l|}{ Race (Ref: non-White) } \\
\hline White & $0.560(0.270)$ & $0.248-1.450$ & 0.256 \\
\hline \multicolumn{4}{|l|}{ State (Ref: WV) } \\
\hline $\mathrm{KY}$ & $1.040(0.189)$ & $0.729-1.485$ & 0.828 \\
\hline County_Smokerate (Ref: $<31 \%$ ) & & & \\
\hline$>=31 \%$ & $0.449(0.081)$ & $0.314-0.640$ & $0.000 *$ \\
\hline \multicolumn{4}{|l|}{ Overall comorbidity } \\
\hline D’Hoore-CCI & $0.976(0.041)$ & $0.890-1.059$ & 0.561 \\
\hline Number of other chronic conditions & $1.433(0.083)$ & $1.280-1.605$ & $0.000 *$ \\
\hline \multicolumn{4}{|l|}{ COPD severity } \\
\hline$\#$ of inhaled SABA canisters & $1.036(0.015)$ & $1.007-1.066$ & $0.014 *$ \\
\hline \# of inhaled IPR canisters & $0.861(0.015)$ & $0.832-0.892$ & $0.000 *$ \\
\hline \# of other SABD prescriptions & $0.936(0.015)$ & $0.907-0.966$ & $0.000 *$ \\
\hline \# of OCS prescriptions & $1.037(0.033)$ & $0.975-1.103$ & 0.245 \\
\hline Use of nebulized SABD & $1.129(0.192)$ & $0.809-1.576$ & 0.475 \\
\hline $\begin{array}{r}\text { Presence of Hosp/ER visits for COPD or } \\
\text { COPD-related comorbid disorders }\end{array}$ & $1.029(0.155)$ & $0.766-1.381$ & 0.850 \\
\hline $\begin{array}{r}\text { \# of Physician visits for COPD or COPD- } \\
\text { related comorbid disorders }\end{array}$ & $1.012(0.020)$ & $0.974-1.053$ & 0.538 \\
\hline Presence of asthma & $1.468(0.295)$ & $0.990-2.178$ & 0.056 \\
\hline Use of Home oxygen therapy & $1.114(0.231)$ & $0.743-1.672$ & 0.600 \\
\hline
\end{tabular}


TABLE B2: Logistic regression predicting the probability of being prescribed ICS vs. SABD

\begin{tabular}{|c|c|c|c|}
\hline \multirow[b]{2}{*}{ Variable } & \multicolumn{2}{|c|}{ DV: Probability of receiving ICS } & \multirow[b]{2}{*}{$\begin{array}{c}p \\
\text { value }\end{array}$} \\
\hline & Odds ratio (SE) & $95 \% C I$ & \\
\hline \multicolumn{4}{|l|}{ Demographic } \\
\hline Age (years) & $0.984(0.007)$ & $0.969-0.999$ & $0.038 *$ \\
\hline Gender (Ref: Male) & & & \\
\hline Female & $1.171(0.136)$ & $0.932-1.472$ & 0.174 \\
\hline Race (Ref: non-White) & & & \\
\hline White & $0.598(0.236)$ & $0.276-1.295$ & 0.192 \\
\hline State (Ref: WV) & & & \\
\hline $\mathrm{KY}$ & $0.764(0.112)$ & $0.573-1.020$ & 0.068 \\
\hline County_Smokerate(Ref: <31\%) & & & \\
\hline$>=31 \%$ & $0.772(0.114)$ & $0.578-1.032$ & 0.081 \\
\hline \multicolumn{4}{|l|}{ Overall comorbidity } \\
\hline D'Hoore-CCI & $0.989(0.035)$ & $0.923-1.060$ & 0.759 \\
\hline Number of other chronic conditions & $1.245(0.060)$ & $1.133-1.368$ & $0.000 *$ \\
\hline \multicolumn{4}{|l|}{ COPD severity } \\
\hline$\#$ of inhaled SABA canisters & $1.047(0.013)$ & $1.023-1.073$ & $0.000 *$ \\
\hline \# of inhaled IPR canisters & $0.910(0.011)$ & $0.888-0.932$ & $0.000^{*}$ \\
\hline \# of other SABD prescriptions & $0.959(0.012)$ & $0.936-0.982$ & $0.000 *$ \\
\hline \# of OCS prescriptions & $0.997(0.025)$ & $0.950-1.046$ & 0.896 \\
\hline Use of nebulized SABD & $0.949(0.131)$ & $0.725-1.243$ & 0.706 \\
\hline $\begin{array}{r}\text { Presence of Hosp/ER visits for COPD or } \\
\text { COPD-related comorbid disorders }\end{array}$ & $1.370(0.165)$ & $1.083-1.734$ & $0.009 *$ \\
\hline $\begin{array}{r}\text { \# of Physician visits for COPD or COPD- } \\
\text { related comorbid disorders }\end{array}$ & $0.993(0.017)$ & $0.960-1.027$ & 0.685 \\
\hline Presence of asthma & $1.385(0.225)$ & $1.007-1.905$ & $0.045^{*}$ \\
\hline Use of Home oxygen therapy & $0.792(0.130)$ & $0.574-1.093$ & 0.156 \\
\hline
\end{tabular}


TABLE B3: Logistic regression predicting the probability of being prescribed ICS/LABA vs. LABA

\begin{tabular}{|c|c|c|c|}
\hline \multirow[b]{2}{*}{ Variable } & \multicolumn{3}{|c|}{ DV: Probability of receiving ICS/LABA } \\
\hline & Odds ratio (SE) & $95 \% C I$ & $\begin{array}{c}p \\
\text { value }\end{array}$ \\
\hline \multicolumn{4}{|l|}{ Demographic } \\
\hline Age (years) & $0.977(0.008)$ & $0.960-0.994$ & $0.010^{*}$ \\
\hline Gender (Ref: Male) & & & \\
\hline Female & $1.175(0.164)$ & $0.893-1.545$ & 0.250 \\
\hline Race (Ref: non-White) & & & \\
\hline White & $0.763(0.303)$ & $0.350-1.663$ & 0.496 \\
\hline State (Ref: WV) & & & \\
\hline $\mathrm{KY}$ & $1.336(0.227)$ & $0.957-1.865$ & 0.089 \\
\hline County_Smokerate (Ref: $<31 \%)$ & & & \\
\hline$>=31 \%$ & $0.674(0.114)$ & $0.485-0.938$ & $0.019 *$ \\
\hline \multicolumn{4}{|l|}{ Overall comorbidity } \\
\hline D’Hoore-CCI & $0.936(0.035)$ & $0.870-1.008$ & 0.079 \\
\hline Number of other chronic conditions & $1.097(0.057)$ & $0.990-1.215$ & 0.076 \\
\hline \multicolumn{4}{|l|}{ COPD severity } \\
\hline \# of inhaled SABA canisters & $0.990(0.010)$ & $0.970-1.010$ & 0.334 \\
\hline \# of inhaled IPR canisters & $0.986(0.015)$ & $0.957-1.015$ & 0.345 \\
\hline \# of other SABD prescriptions & $1.008(0.017)$ & $0.975-1.042$ & 0.651 \\
\hline \# of OCS prescriptions & $0.985(0.028)$ & $0.931-1.042$ & 0.608 \\
\hline Use of nebulized SABD & $1.740(0.303)$ & $1.237-2.449$ & $0.001 *$ \\
\hline $\begin{array}{r}\text { Presence of Hosp/ER visits for COPD or } \\
\text { COPD-related comorbid disorders }\end{array}$ & $1.074(0.153)$ & $0.813-1.420$ & 0.614 \\
\hline $\begin{array}{r}\text { \# of Physician visits for COPD or COPD- } \\
\text { related comorbid disorders }\end{array}$ & $1.024(0.020)$ & $0.985-1.065$ & 0.227 \\
\hline Presence of asthma & $1.320(0.246)$ & $0.916-1.902$ & 0.137 \\
\hline Use of Home oxygen therapy & $1.049(0.205)$ & $0.715-1.538$ & 0.808 \\
\hline
\end{tabular}




\author{
ANNA O. D'SOUZA \\ adsouza@hsc.wvu.edu \\ Department of Pharmaceutical Systems \& Policy \\ P.O. Box 9510 \\ Morgantown, WV 26505 \\ Phone: (304) 685-3661
}

\title{
CAREER OBJECTIVE
}

To conduct research in the areas of Health Outcomes (outcomes of drug use and clinical interventions) and Health Policy (impact of prescription drug policy on patterns of care).

\section{EDUCATION \& FELLOWSHIP}

2001 - 2006 Doctor of Philosophy, Pharmaceutical Systems and Policy 2001-2003 Aventis Health Outcomes Research Fellowship, West Virginia University Dissertation title: Clinically relevant and economic outcomes of maintenance pharmacotherapy in chronic obstructive pulmonary disease (COPD)

1999 - $2001 \quad$ M.S., Pharmacy Administration The University of Toledo, Ohio Thesis title: Perceptions of physicians regarding the effects of direct-toconsumer advertising of prescription drugs

Bachelor of Pharmacy (B.Pharm.)

1995 - 1999 Bharati Vidyapeeth College of Pharmacy

University of Mumbai, India

\section{RESEARCH EXPERIENCE}

Projects as Aventis Fellow

1. Aventis-Barbara Davis Center for Adult and Childhood Diabetes (BDC) [Mentor: Laura Menditto, MPH, MBA, November 2002-November 2003]

- Provided input for the research design and PRO section of the data collection instrument and conducted analysis of data to determine clinical and patient-reported outcome of a new insulin analogue

2. Rheumatoid Arthritis (RA)-Pharmacoeconomics [Mentor: Joseph Doyle, R.Ph., MPH, November 2002-May 2003]

- Conducted a literature review and combined data from a prior project to determine most costeffective option

3. Patient Preferences for Drug Product Attributes [Mentor: Leon Dupclay, Ph.D. R.Ph, January 2003-March 2003]

- Conducted literature review to create a conceptual framework that outlines factors affecting patient preferences for drug products based solely on product attributes 
Projects as WVU Research Assistant

1. WVU Company-Sponsored Project in Overactive Bladder (OAB) [September 2005-September 2006]

- Developed study design, analyzed data, and prepared poster and manuscript of project assessing persistence, adherence, and switch patterns of drugs for overactive bladder

2. Project using the Medical Expenditure Panel Survey Dataset [July 2005-Present]

- Developed study concept, design, and analyzed data to determine the impact of burden of outof-pocket health and prescription drug costs

3. Burden of Asthma in the West Virginia Medicaid Population [June 2003-October 2003]

- Conducted a statewide asthma surveillance of asthma-related morbidity in the West Virginia Medicaid population

4. Pharmacist Shortage in West Virginia: Survey of Pharmacists-in-Charge and Pharmacists [August 2001-November 2001]

- Processed data from self-administered mail surveys and conducted analysis for the State of WV regarding pharmacist workforce

\section{TEACHING EXPERIENCE}

1. Teaching Assistant at the School of Pharmacy, West Virginia University [2003-2004]

- Outcomes Assessment \& Quality Improvement: Responsibilities included grading, preparing handouts, and lecturing (Lecture on Introduction to Pharmacoepidemiology)

2. Teaching Assistant at the School of Pharmacy, University of Toledo [1999-2001]

- Pharmaceutical Marketing \& Management: Responsibilities included grading, preparing handouts, and lecturing.

- Health care systems: Responsibilities included grading and preparing handouts

\section{Publications}

D’Souza AO, Smith MJ, Miller LA, Doyle J, Ariely R, Persistence, adherence, and switch rates among long-acting and short-acting over-active bladder (OAB) medications in a regional managed care plan (Under Review: Journal of Managed Care Pharmacy)

D'Souza, AO, Smith, MJ, Miller, LA, \& Kavookjian, J (2006). An appraisal of pharmacoeconomic evidence of maintenance therapy for COPD. Chest, 129, 1693-1708.

D'Souza AO, Miller LA. Measuring adherence to antihypertensive drug therapy. J Manag Care Pharm 2004; 10(1):88-89.

Garg SK, Walker AJ, Hoff HK, D'Souza AO, Gottlieb PA, Chase HP. Glycemic parameters with multiple daily injections using insulin glargine versus insulin pump. Diabetes Technol Ther 2004; 6(1):9-15.

Garg SK, Gottlieb PA, Hisatomi ME, D'Souza A, Walker AJ, Izuora KE et al. Improved glycemic control without an increase in severe hypoglycemic episodes in intensively treated patients with type 
1 diabetes receiving morning, evening, or split dose insulin glargine. Diabetes Res Clin Pract 2004; 66(1):49-56.

Joshi AV, D'Souza AO, Madhavan SS. Differences in hospital length-of-stay, charges, and mortality in congestive heart failure patients. Congest Heart Fail 2004; 10(2):76-84.

Smith MJ, D'Souza AO (2003) Rates of asthma and asthma-related prescription and medical services within the West Virginia Medicaid population", Grant/Contract number: G031132: West Virginia State Government Statistical Brief.

D'Souza A, Lively B, Siganga W, Goodman M. Effects of direct-to-consumer advertising of prescription drugs: Perceptions of primary care physicians. J Pharm Mktg Mgmt 2003; 15:61-75.

\section{PRESENTATIONS}

D’Souza AO, Smith M, Roy AN, "Patterns of Pharmacotherapy Utilization among Children and Adolescents with Multiple Asthma-related morbid Events Enrolled in a Medicaid Program", $10^{\text {th }}$ Annual ISPOR Meeting, May $15^{\text {th }}-18^{\text {th }}, 2005$, Washington, DC.

D’Souza AO, Smith M, “Costs for Asthma-Related Medical Services and Prescription Medications in a State Medicaid Program",

$9^{\text {th }}$ Annual ISPOR Meeting, May $16^{\text {th }}-19^{\text {th }}, 2004$, Arlington, VA

$40^{\text {th }}$ Annual DIA Meeting, June $13^{\text {th }}-17^{\text {th }}, 2004$, Washington D.C.

D’Souza AO, Crivera C, Doyle JJ, "Economic Analysis of Novel Disease Modifying Anti-Rheumatic Drugs in a Managed Care Setting", $8^{\text {th }}$ International Society for Pharmacoeconomics and Outcomes Research (ISPOR) Annual Meeting, May $17^{\text {th }}-22^{\text {nd }} 2003$, Arlington, VA.

D'Souza AO, Gottlieb PA, Hisatomi ME, Hoff HK, Izuora KE, Walker AJ, Chase PH, Tibbets CM, Antell L, Garg SK, "Improved Glycemic control and Reduced Severe Hypoglycemic Episodes in Adults with Type 1 Diabetes Intensively Treated with Insulin Glargine", $18^{\text {th }}$ International Diabetes Federation Congress, August $24^{\text {th }}-29^{\text {th }} 2003$, Paris, France.

Hoff HK, Gottlieb PA, Hisatomi ME, Izuora KE, Chase PH, Tibbets CM, D’Souza AO, Garg SK, "Basal-Bolus Programme using Multiple Daily Insulin Injections versus External Insulin Infusion Pump: Glycemic Parameters in Adults with Type 1 Diabetes", $18^{\text {th }}$ International Diabetes Federation Congress, August $24^{\text {th }}-29^{\text {th }} 2003$, Paris, France.

Garg SK, Hisatomi ME, D’Souza AO, “Impact of Morning vs. Evening or Split Insulin Glargine Usage on Glycemic Parameters in Intensively Treated Adults with Type 1 Diabetes", $63^{\text {rd }}$ Scientific Sessions, American Diabetes Association, June $13^{\text {th }}-17^{\text {th }} 2003$, New Orleans, LA.

D’Souza AO, Madhavan SS, "Diabetes-Related Drug Utilization and Expenditures in Medicare Beneficiaries with and without Supplemental Prescription Drug Insurance", 8 th $I S P O R$ Annual Meeting, May $17^{\text {th }}-22^{\text {nd }} 2003$, Arlington, VA.

Joshi AV, D'Souza AO, Madhavan SS. "Differences in Hospital Length-of-Stay, Charges, and Mortality in Congestive Heart Failure Patients", $7^{\text {th }}$ ISPOR Annual Meeting, May $19^{\text {th }}-22^{\text {nd }} 2002$, Arlington, VA. 
D’Souza AO, Lively BT, Siganga W, Goodman HG, "Perceptions of Physicians Regarding the Effects of Direct-to-Consumer Advertising of Prescription Drugs", $150^{\text {th }}$ American Pharmacists Association Annual Meeting, March $15^{\mathrm{h}}-19^{\text {th }}$ 2002, Philadelphia, PA.

D'Souza AO, Deshmukh H, Singh R, Siganga W, "Evaluation of the Need to Enhance Computer Application Skills of Fourth Year Pharmacy Students during their Pharmacy Education", $149^{\text {th }}$

American Pharmacists Association Annual Meeting, March $16^{\text {th }}-20^{\text {th }} 2001$, San Francisco, California.

\section{OTHER PROFESSIONAL EXPERIENCE}

Pharmacy Technician Intern, The Pharm, Toledo, Ohio. [May 2001- July 2001]

Summer Intern, German Remedies Ltd., Mumbai, India [June 1998- July 1998]

Peer Reviewer

- Journal of Pharmaceutical Marketing and Management

- Research in Social and Administrative Pharmacy

- Chest

\section{AWARDS \& HONORS}

2003 Best Student Poster Award, $8^{\text {th }}$ Annual ISPOR meeting 2002 APhA-APPM Presentation Merit Award 2002 Rho Chi Pharmacy Honor Society 1999-2001 Teaching Assistantship, University of Toledo, Ohio Best Student of the Year 1998-99, Bharati Vidyapeeth College of Pharmacy, Mumbai, India

\section{AfFiliations}

2000-2005: International Society for Pharmacoeconomics and Outcomes Research (ISPOR) 2002-2003: President (WVU-ISPOR student chapter)

2001-2002: Treasurer (WVU-ISPOR student chapter)

2000-2002: American Pharmacists Association (APhA) 


\begin{tabular}{|c|c|c|}
\hline REFERENCES & $\begin{array}{l}\text { - Michael James Smith, Ph.D., R.Ph. } \\
\text { Ph.D. Advisor, Assistant Professor } \\
\text { Pharmaceutical Systems \& Policy } \\
\text { P.O. Box 9510, Morgantown, WV } \\
\text { 26506. Phone: (304) 293-1832 } \\
\text { Email: msmith2@hsc.wvu.edu } \\
\text { - Suresh Madhavan, Ph.D., MBA } \\
\text { Ph.D. Committee Member, Professor } \\
\text { Pharmaceutical Systems \& Policy } \\
\text { P.O. Box 9510, Morgantown, WV } \\
\text { 26506. } \\
\text { Phone: (304) 293-1652 } \\
\text { Email: smadhavan@hsc.wvu.edu }\end{array}$ & $\begin{array}{l}\text { - Lesley-Ann Miller, Ph.D. } \\
\text { Ph.D. Committee Member, Assistant } \\
\text { Professor } \\
\text { Pharmaceutical Systems \& Policy } \\
\text { P.O. Box 9510, Morgantown, WV } \\
\text { 26506. } \\
\text { Phone: (304) 293-0228 } \\
\text { Email: lamiller@hsc.wvu.edu } \\
\text { - Laura Menditto MBA, MPH } \\
\text { Ph.D. Committee Member } \\
\text { Director(CVM/Oncology US } \\
\text { Health Outcomes) } \\
\text { GlaxoSmithKline } \\
\text { 200 North 16th Street } \\
\text { Box 7929 (Mail Code FP1280) } \\
\text { Philadelphia, PA 19102 } \\
\text { Phone: (215) 751-6341 } \\
\text { Email: laura.a.menditto@gsk.com }\end{array}$ \\
\hline $\begin{array}{l}\text { RELEVANT } \\
\text { GRADUATE } \\
\text { COURSEWORK }\end{array}$ & $\begin{array}{l}\text { Epidemiology } \\
\text { - Epidemiology } \\
\text { - Pharmacoepidemiology } \\
\text { - Epidemiology of Chronic Diseases } \\
\text { Research Methods } \\
\text { - Research Methods } \\
\text { - Survey Research Methods } \\
\text { - Data Analysis } \\
\text { - Biannual Seminars in Outcomes } \\
\text { Research } \\
\text { Statistics } \\
\text { - Quantitative Methods I \& II } \\
\text { - Multivariate Analysis } \\
\text { - Advanced Biostatistics } \\
\text { - Econometrics }\end{array}$ & $\begin{array}{l}\text { Essential Electives } \\
\text { - Pharmacoeconomics } \\
\text { - Decision Analysis in Health Care } \\
\text { - Pharmaceutical Marketing \& } \\
\text { Management } \\
\text { - Health Care Law } \\
\text { - Health Care Finance } \\
\text { - Social \& Behavioral Theory in } \\
\text { Outcomes Research } \\
\text { Short Courses taken at ISPOR annual } \\
\text { meetings } \\
\text { - Retrospective Database Analysis } \\
\text { [May 2005] } \\
\text { - Statistical Considerations in } \\
\text { Economic Evaluations [May 2004] } \\
\text { - Cost Estimation and Assessing } \\
\text { Financial (Budget) Impact of New } \\
\text { HealthCare Technologies [May } \\
\text { 2003] }\end{array}$ \\
\hline
\end{tabular}

COMPUTER Proficient in: SAS, STATA, SPSS, FoxPro

SKILLS

Familiar with: TreeAge Pro, Microsoft Access 